\title{
Synthesis and Evaluation of Bioorthogonal Pantetheine Analogs for In Vivo Protein Modification
}

Jordan L. Meier, Andrew C. Mercer, Heriberto Rivera Jr., and Michael D. Burkart* Department of Chemistry and Biochemistry, University of California, San Diego, 9500 Gilman Drive, La Jolla, California 92093-0358

mburkart@ucsd.edu

\section{Supporting Information II}

\section{Contents}

\section{${ }^{1}$ H-NMR Spectra and Enlargements of Single Diastereomers Resulting from Microwave Reactions}

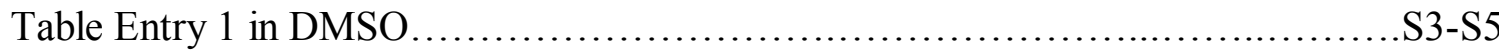

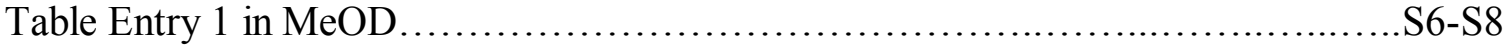

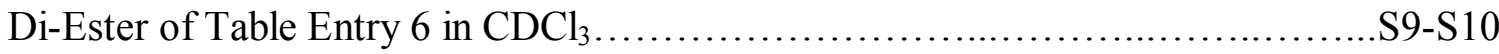

\section{Mass Spectrometry Data}

Mass Spectrometry Data For In Vitro/In Vivo Assays of 1-8, 9-18 and Click Reactions of 1-8.

${ }^{1} \mathrm{H}$ and ${ }^{13} \mathrm{C}-\mathrm{NMR}$ Spectra of 1-18 and all synthetic intermediates

Compound 1/Intermediates $. \mathrm{S} 33-\mathrm{S} 34$

Compound 2/Intermediates S35-S36

Compound 3/Intermediates S37-S40

Compound 4/Intermediates S40-S44

Compound 5/Intermediates .S45-S48

Compound 6/Intermediates .S49-S54 
Compound 7/Intermediates

S55-S56

Compound 8/Intermediates.

S57-S58

Compound 10/Intermediates. S59-S60

Compound 11/Intermediates. S61-S62

Compound 12/Intermediates. .S63-S64

Compound 13/Intermediates. .S65-S68

Compound 15/Intermediates. S69-S76

Compound 16/Intermediates S77-S83

Compound 17/Intermediates. S84-S89

Compound 18/Intermediates. S90-S95

Linker 48/Intermediates S96-S101 
Relax. de lay $1.000 \mathrm{sec}$ Pulse 45.3 degrees

Acq. time $1.993 \mathrm{sec}$

Width 6006 . O Hz

OBSERYE DATA PROCESSING FT size 32768

Total time $53 \mathrm{~min}, 49 \mathrm{sec}$
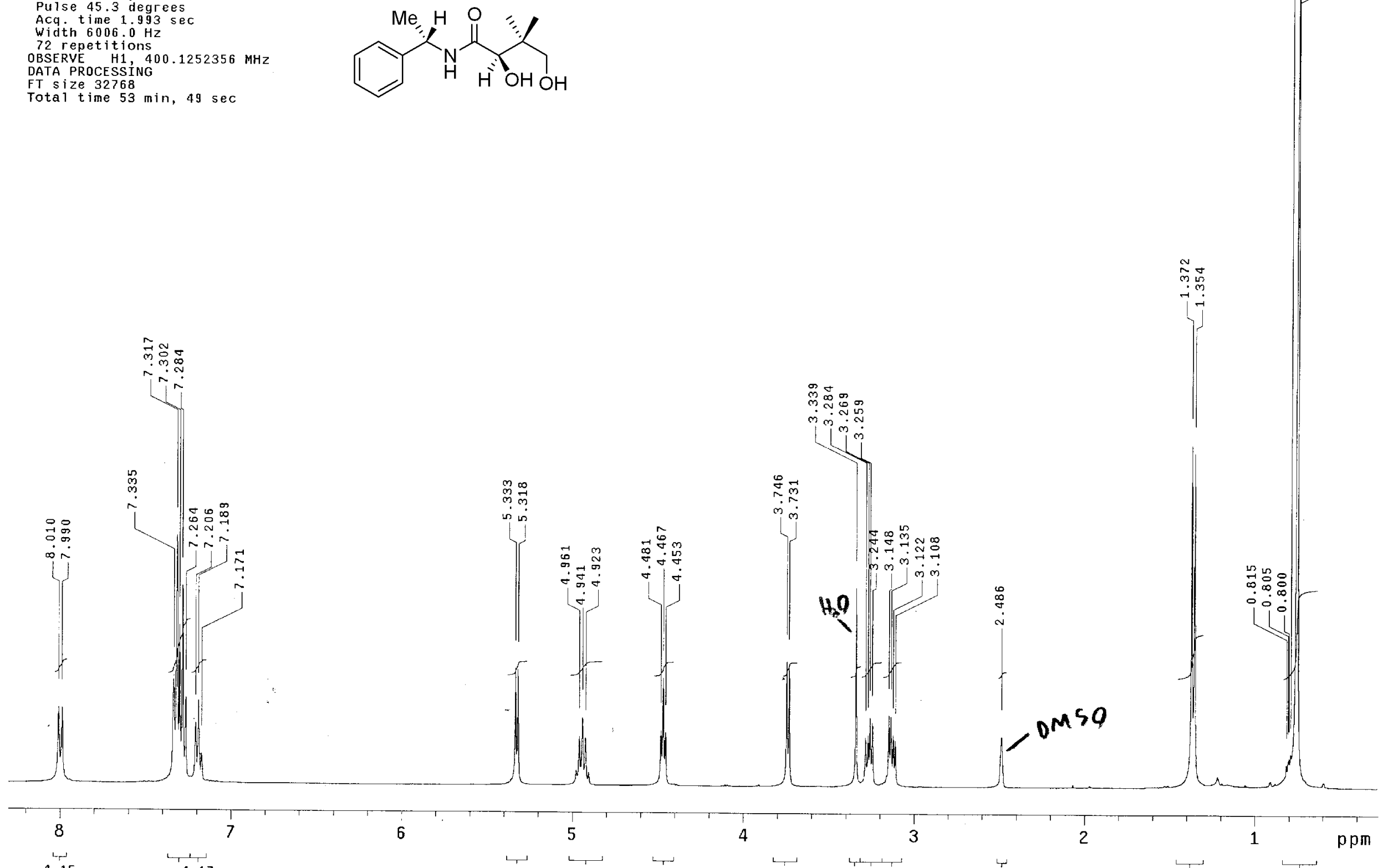
j 1m3-78-ch i ra lam inem i crowave int TOH-DMSOHNMR-04-19-06

Pulse Sequence: s2pul

Solvent: DMso

Ambient temperature
Mercury-400BB "hg 402 "

Relax. delay $1.000 \mathrm{sec}$

Pulse 45.3 degrees

Ac. time 1.993
Width $6006.0 \mathrm{~Hz}$

88 repetitions

OBSERVE HI, $400.1252356 \mathrm{MHz}$
DATA PROCESSING

DATA PROCESSING
FT Size 32768

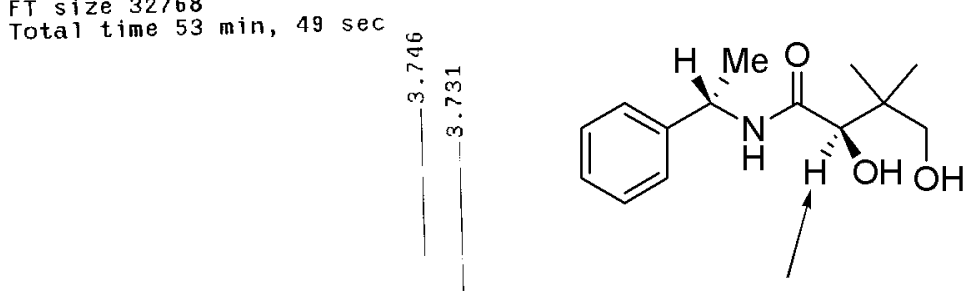

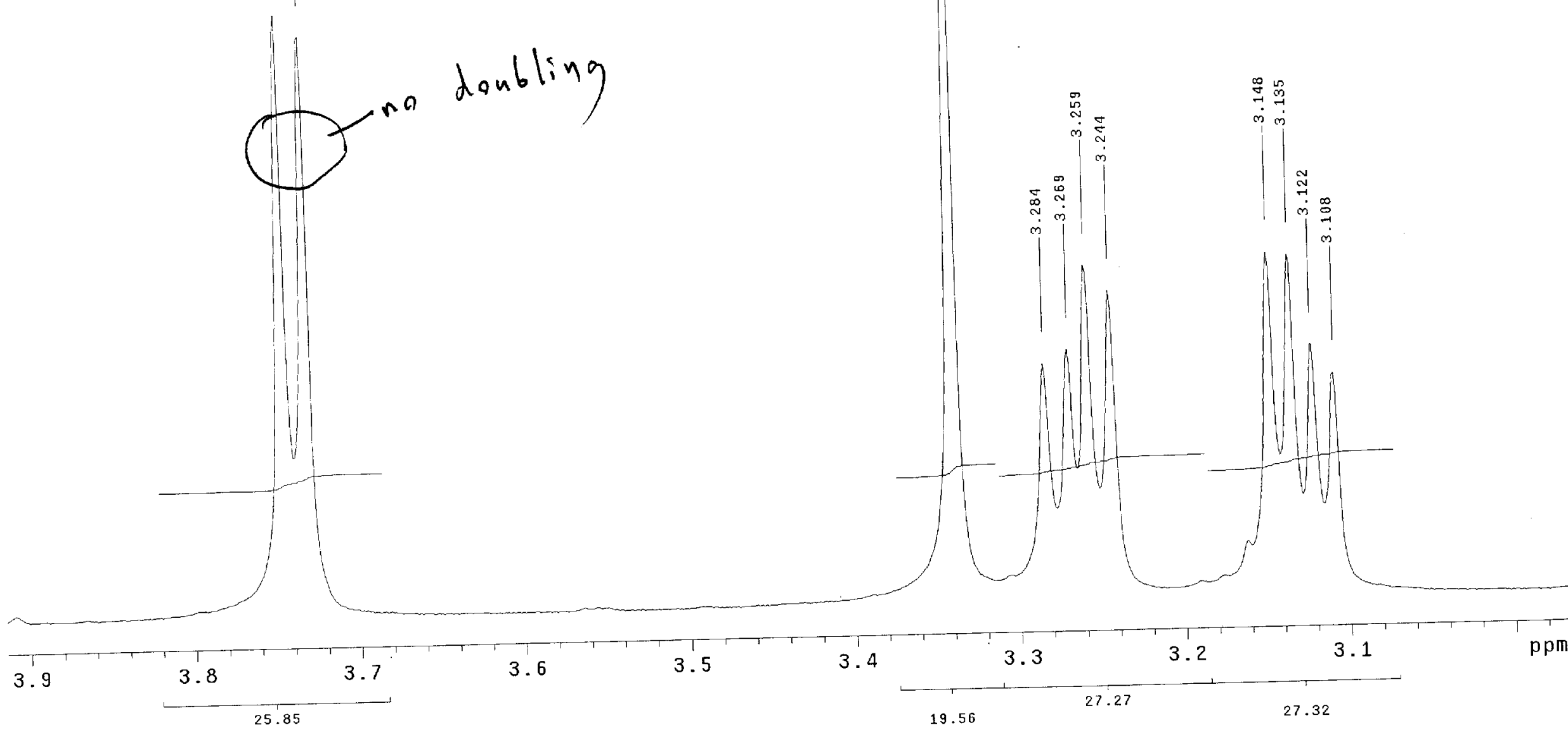


Acq. time 1.993

Width 6006 ions

48 repet H1 400.1249114 MHZ DATA PROCESSING
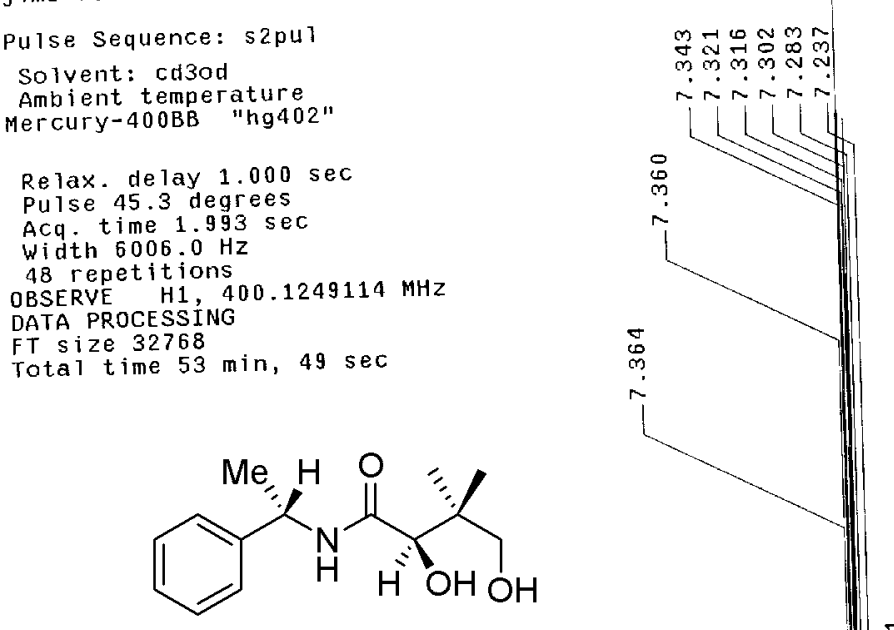

$\left.\left.\approx\right|_{\infty}\right|_{\infty}$
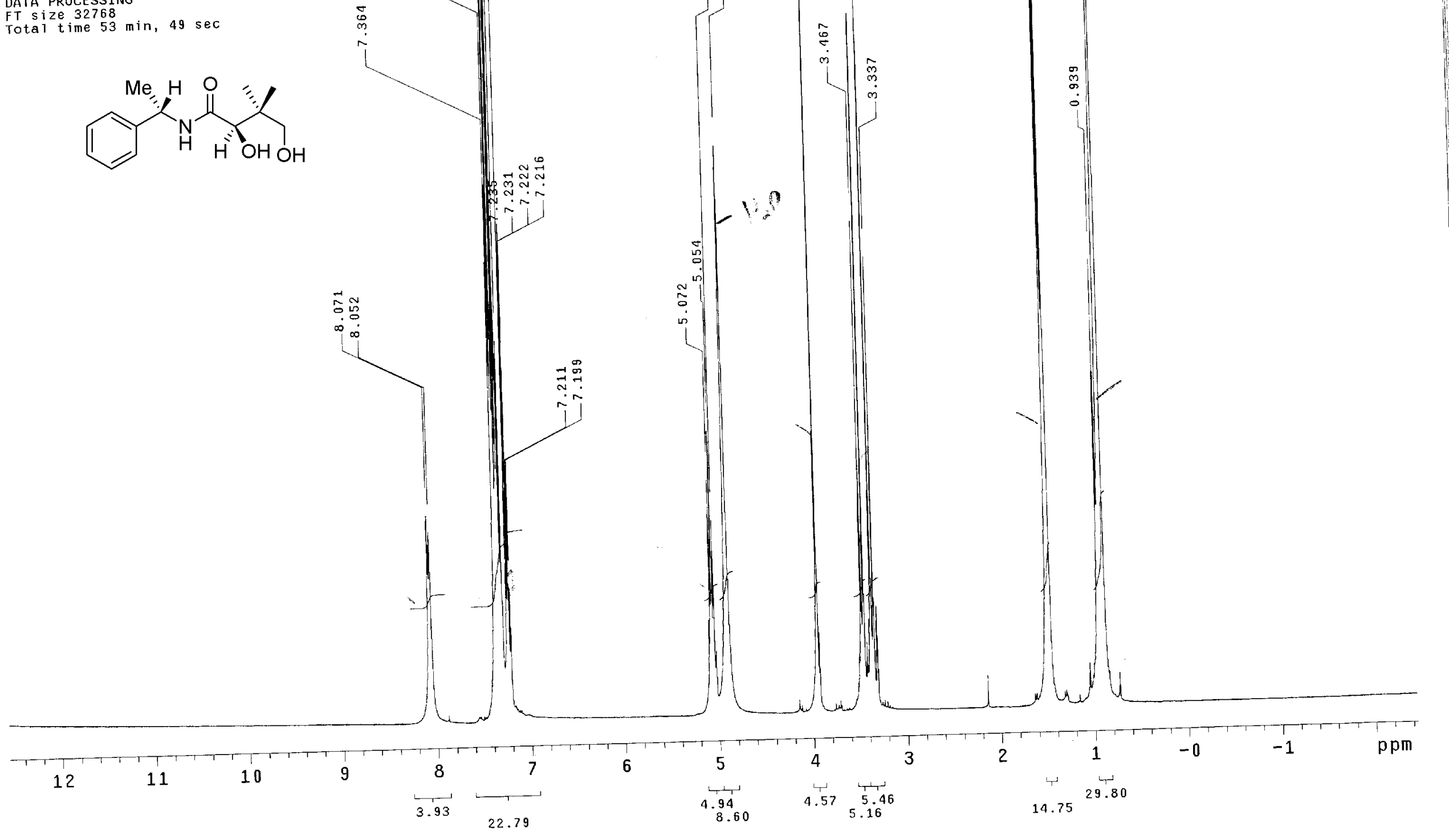


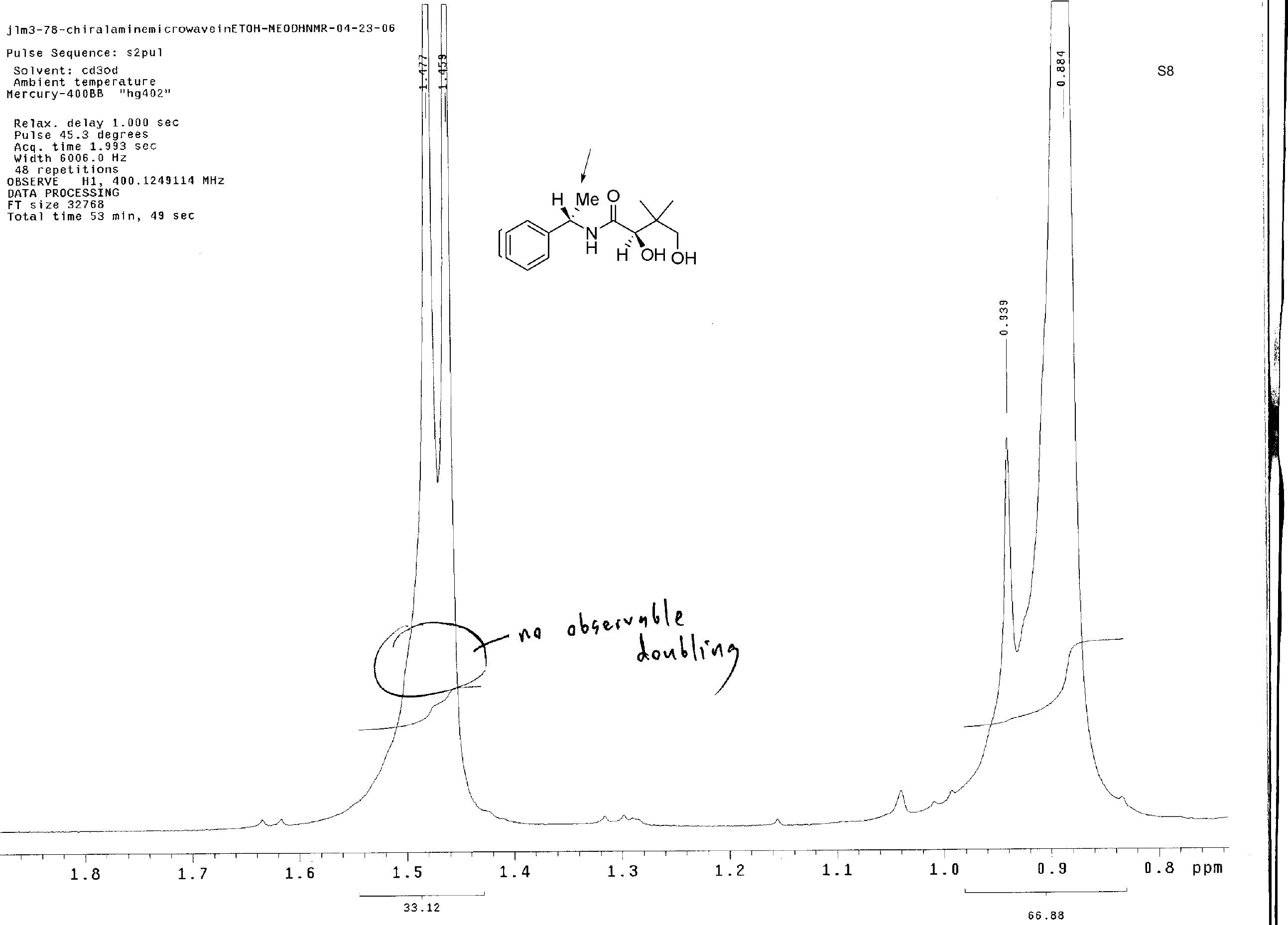


\&

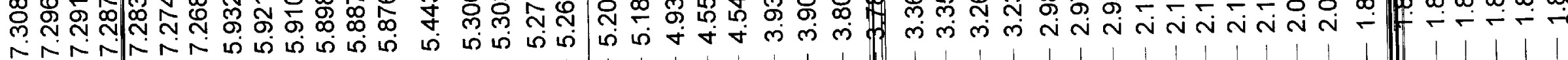

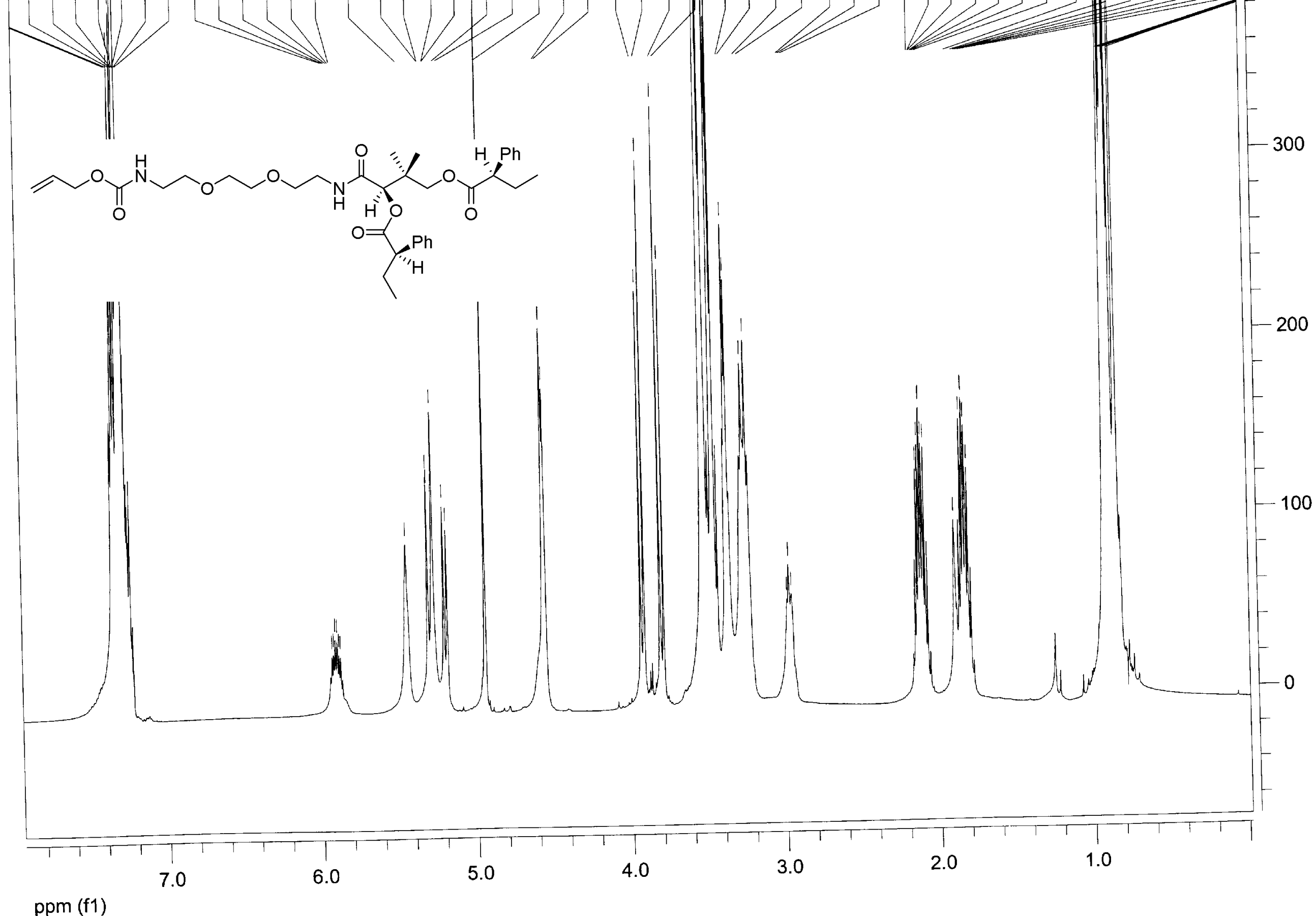

ppm (f1) 


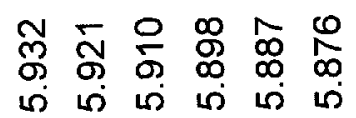

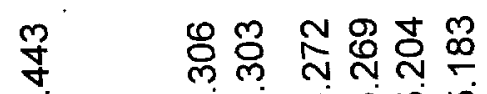

ڤ్

$|+|$

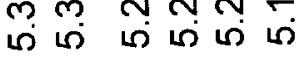
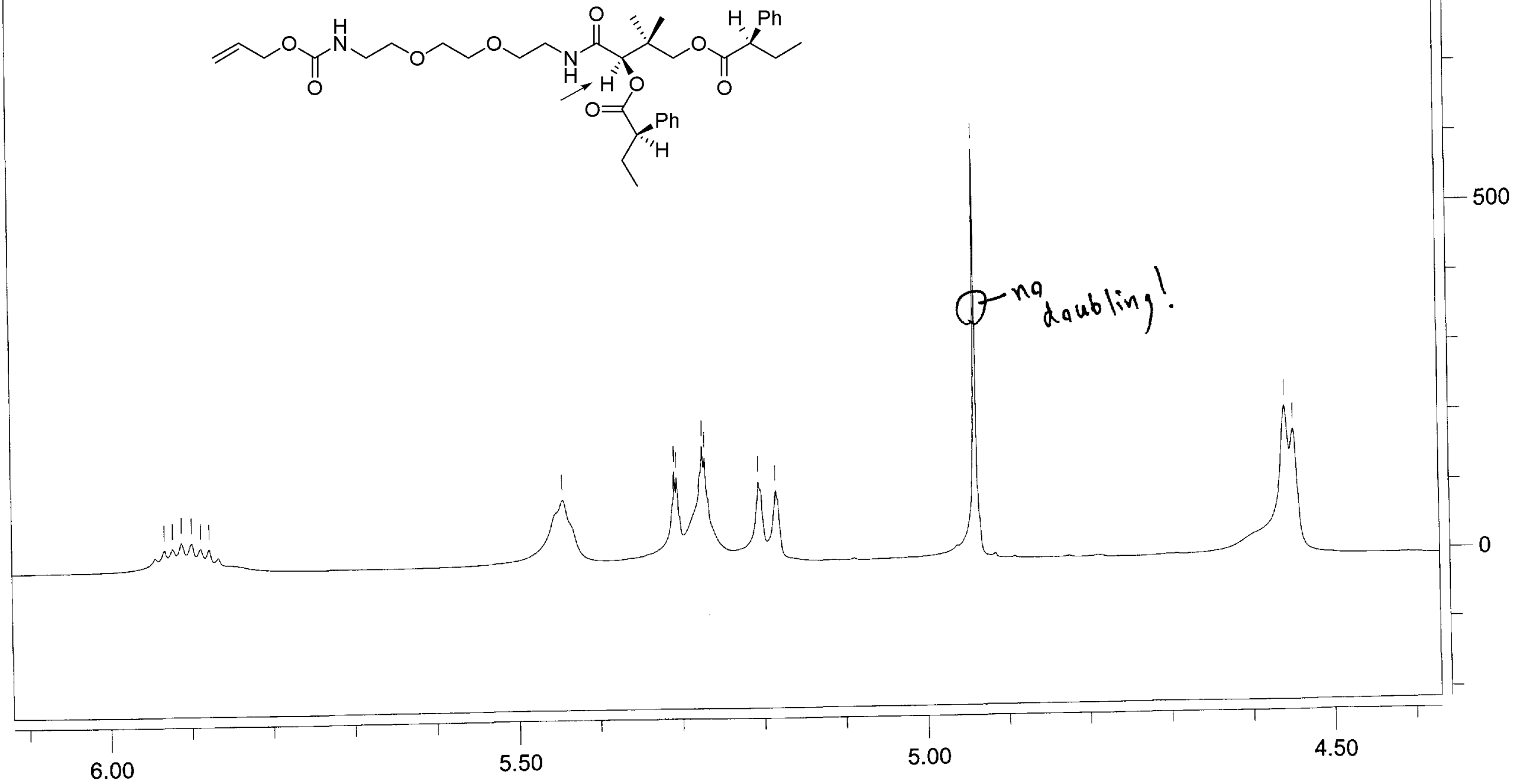
ppm (f1) 


\section{Mass Spectrometry Data}

Protein mass spectrometry was performed using MALDI on an Applied Biosystems Voyager DE STR 4140. Samples were prepared in the same manner in both the in vivo and in vitro assays. For MALDI analysis doubly transformed BL21 E. coli with Fren (frenolicin synthase carrier protein from Streptomyces roseofulvus) and Sfp was used. Samples were mixed 1:5 with the ionization buffer (saturated sinapinc acid in 1:1:1 acetonitrile, TFA, water). A standard deviation of $+/-5$ Da was observed over the course of $20+$ runs with regards to the apo-forms of ACP and Fren. However within each run the mass shift between the apo-form and subsequent crypto-forms was consistent as described in text.

Compound 1 In vitro

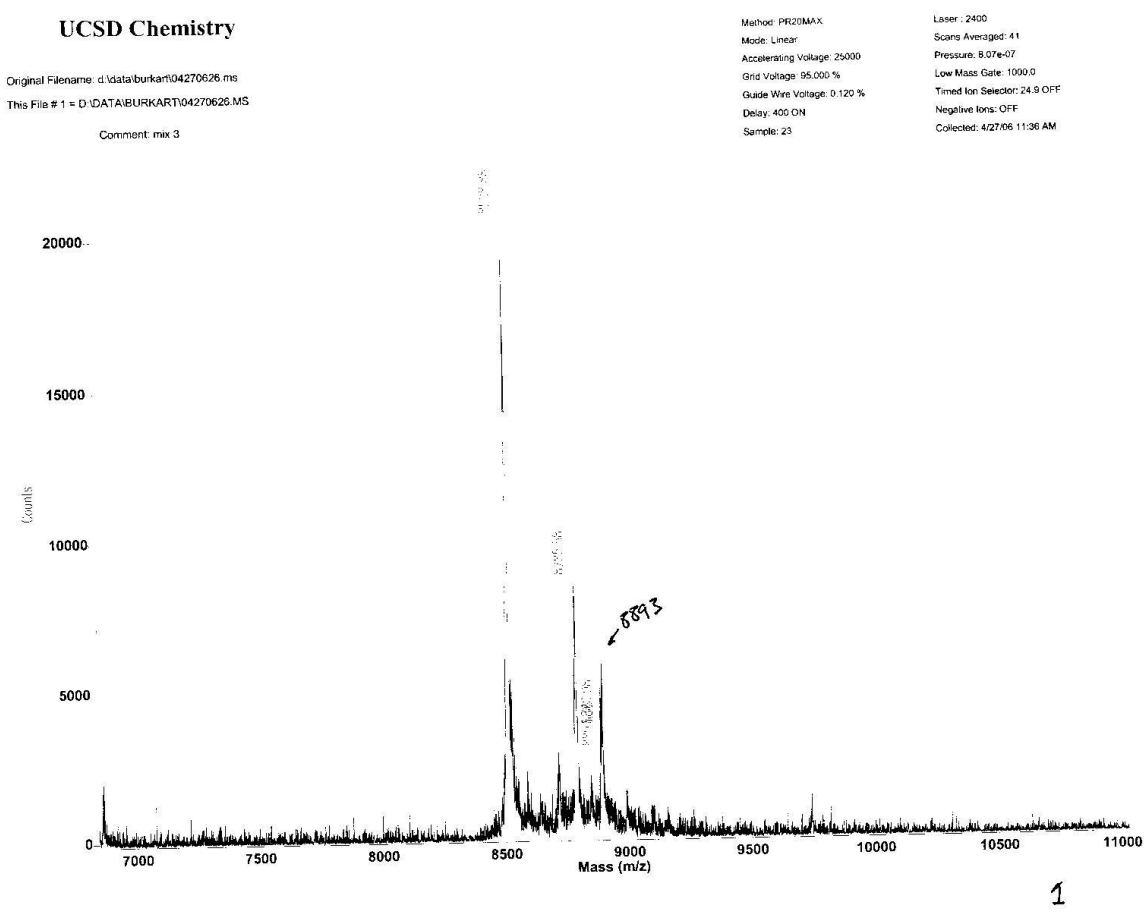


Compound $\mathbf{1}$ In vitro after click reaction with $\mathbf{1 0}$
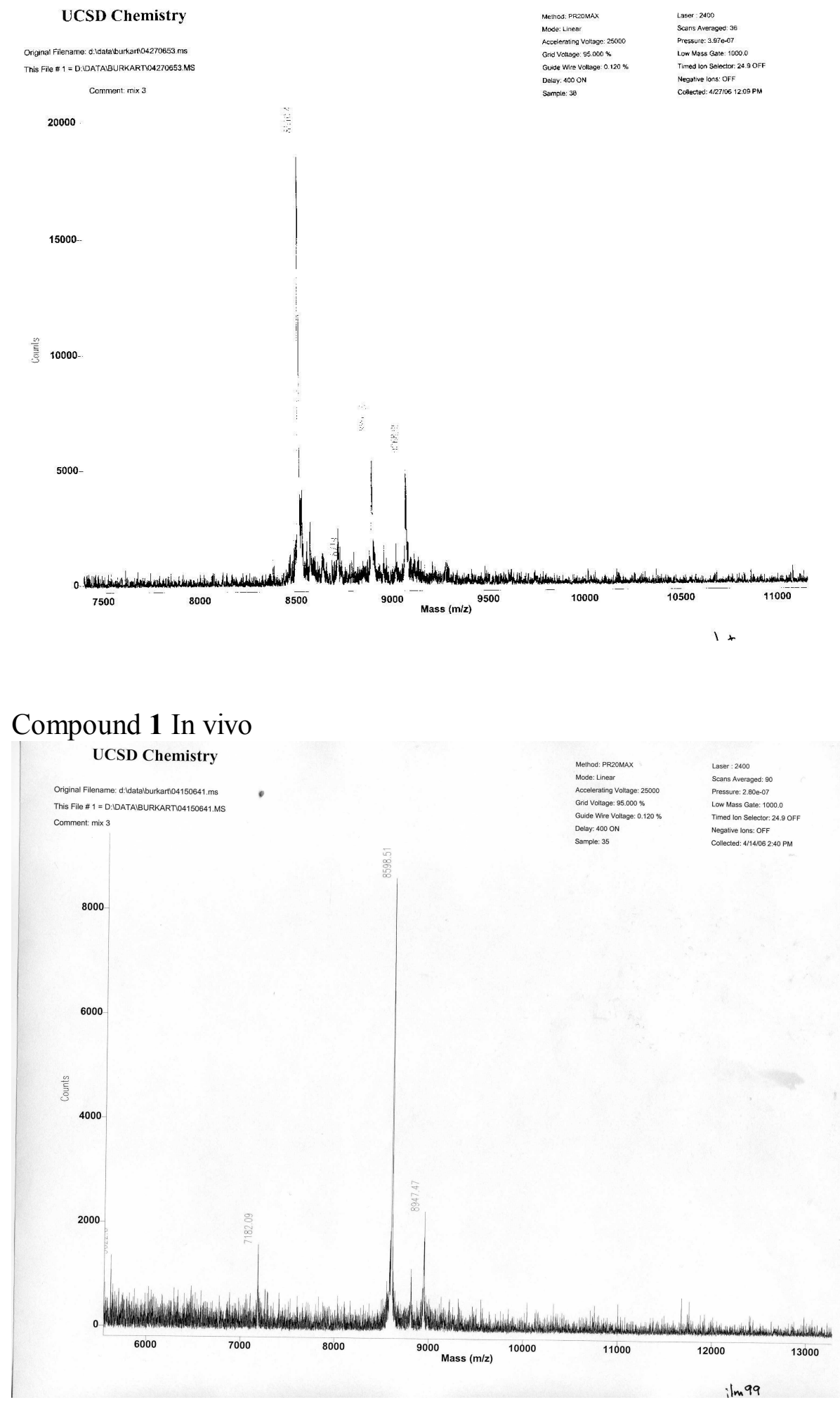
Compound $\mathbf{1}$ In vivo after click reaction with $\mathbf{1 0}$

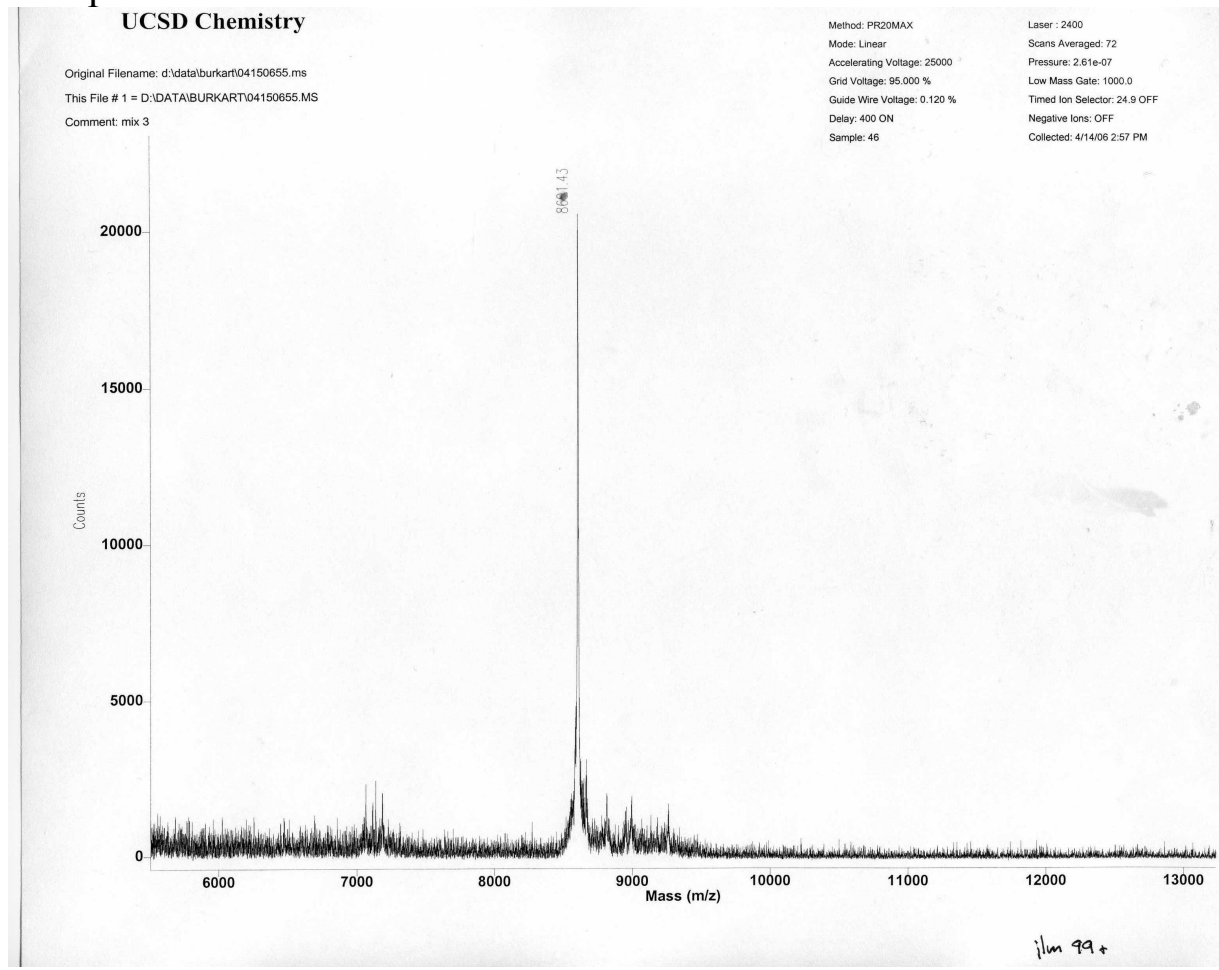

Compound 2 In vitro

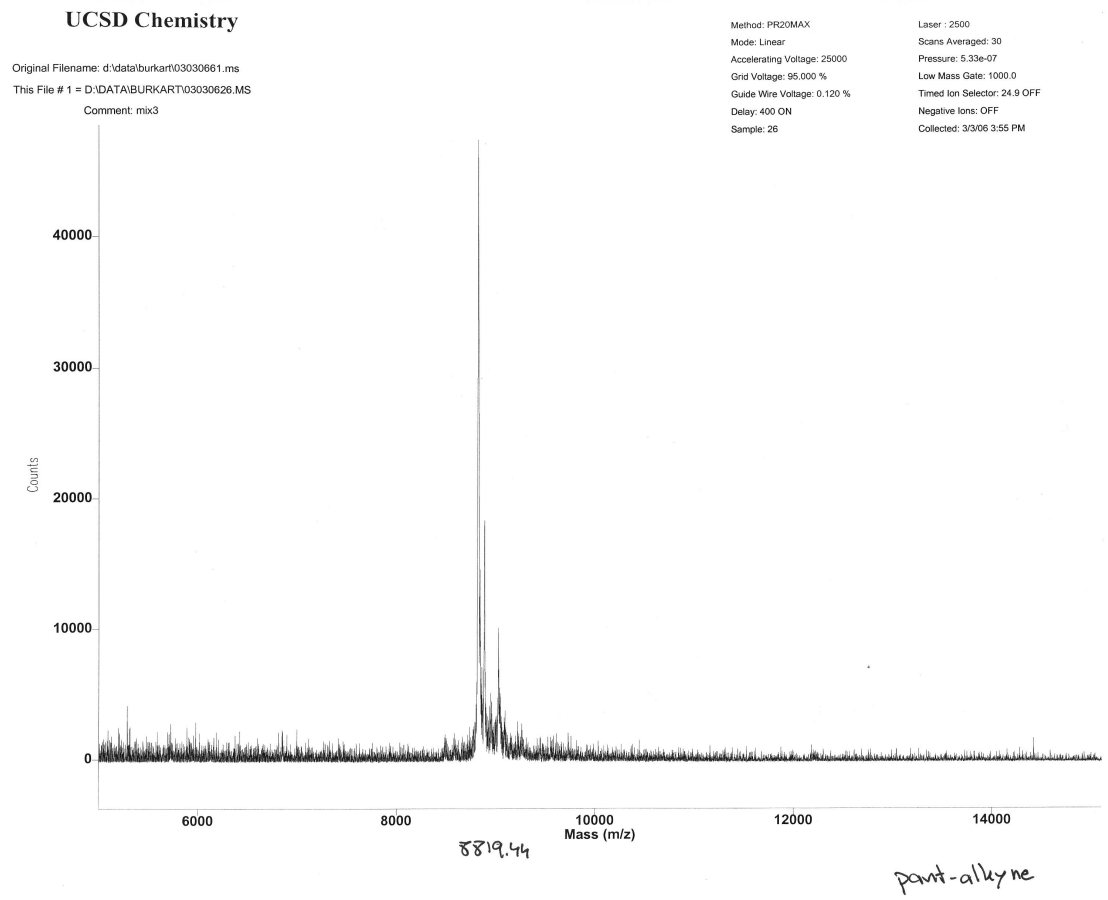


Compound 2 In vitro after click reaction with 11

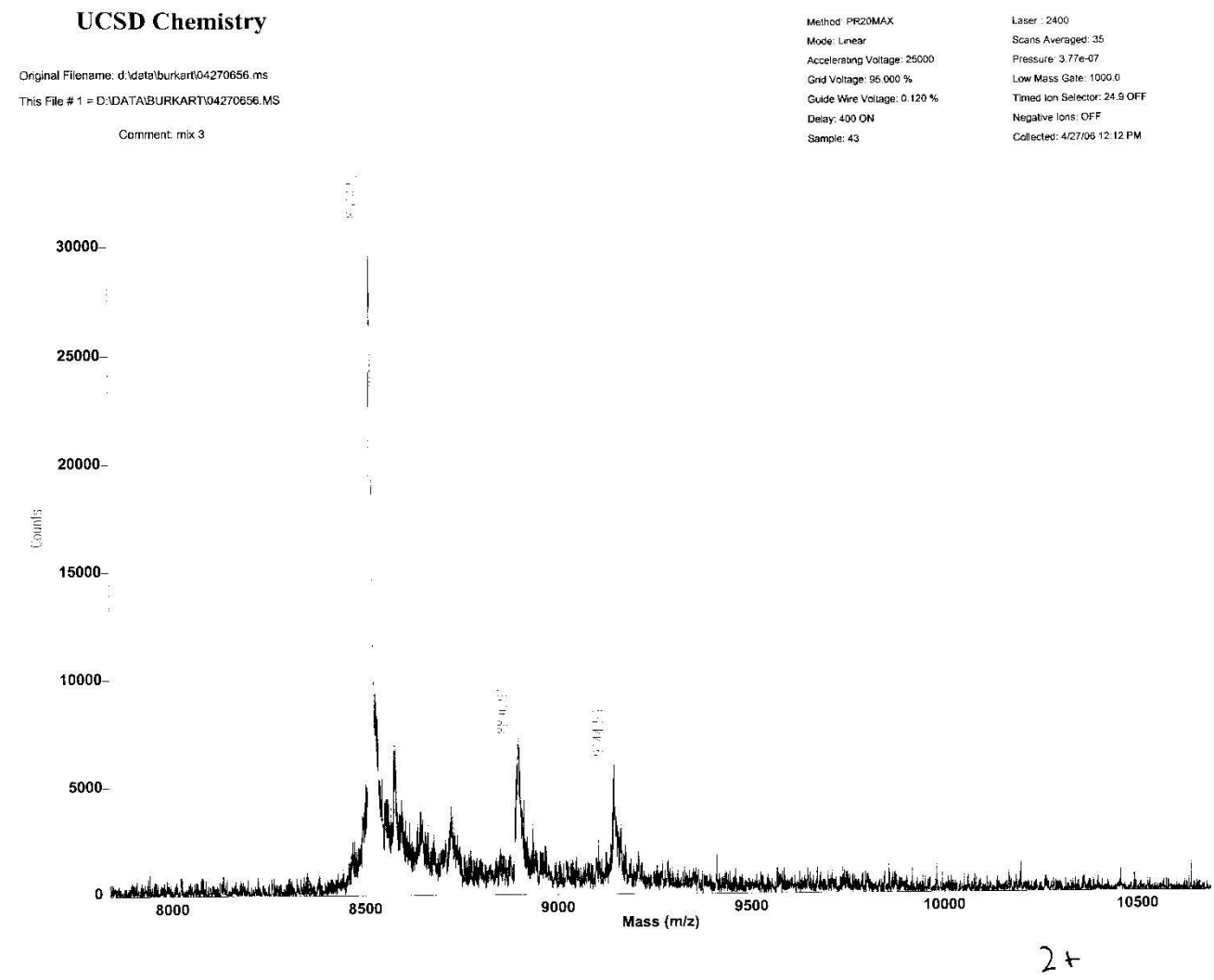

Compound 2 In vivo

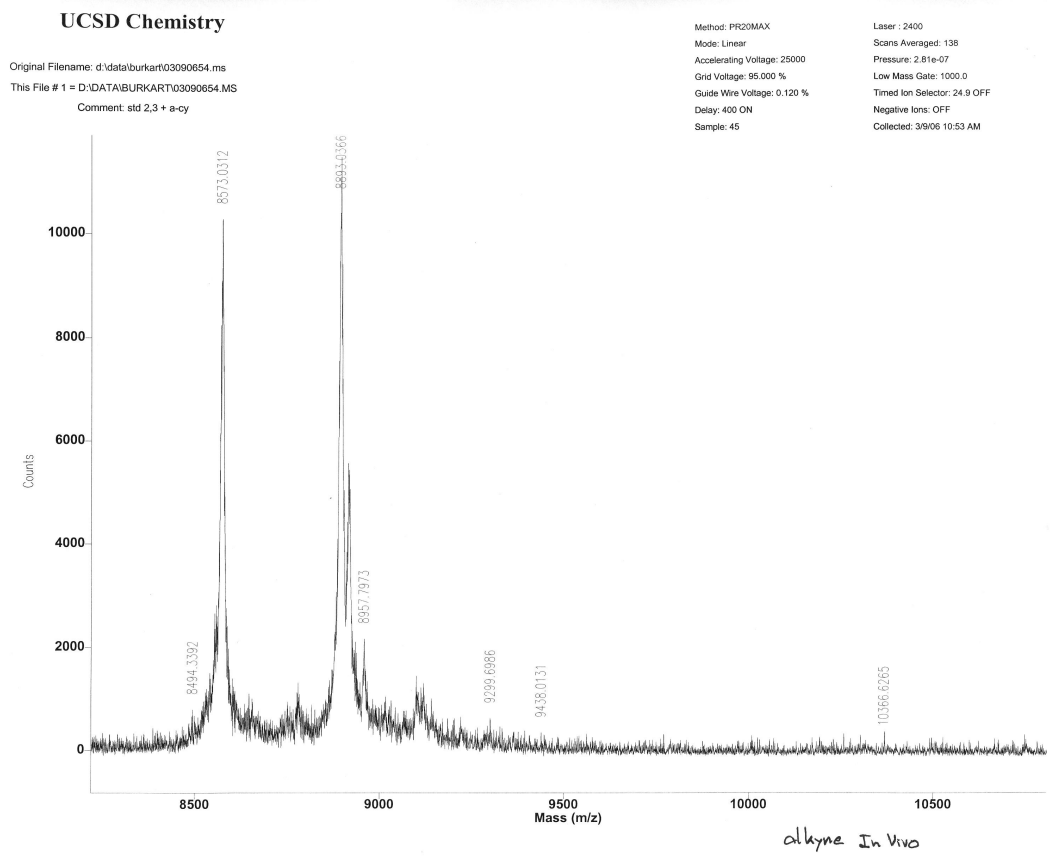


Compound 2 In vivo after click reaction with 11

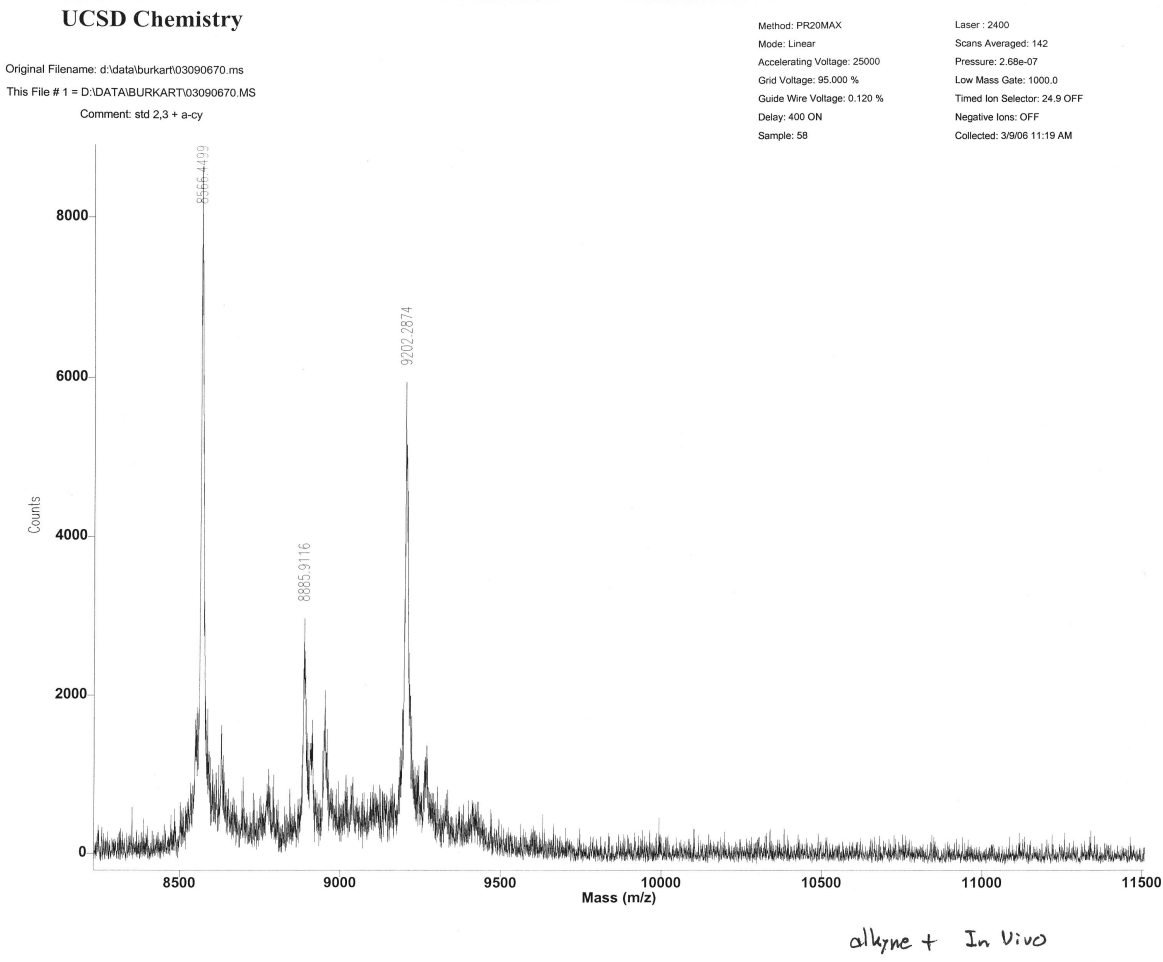

Compound 3 In vitro

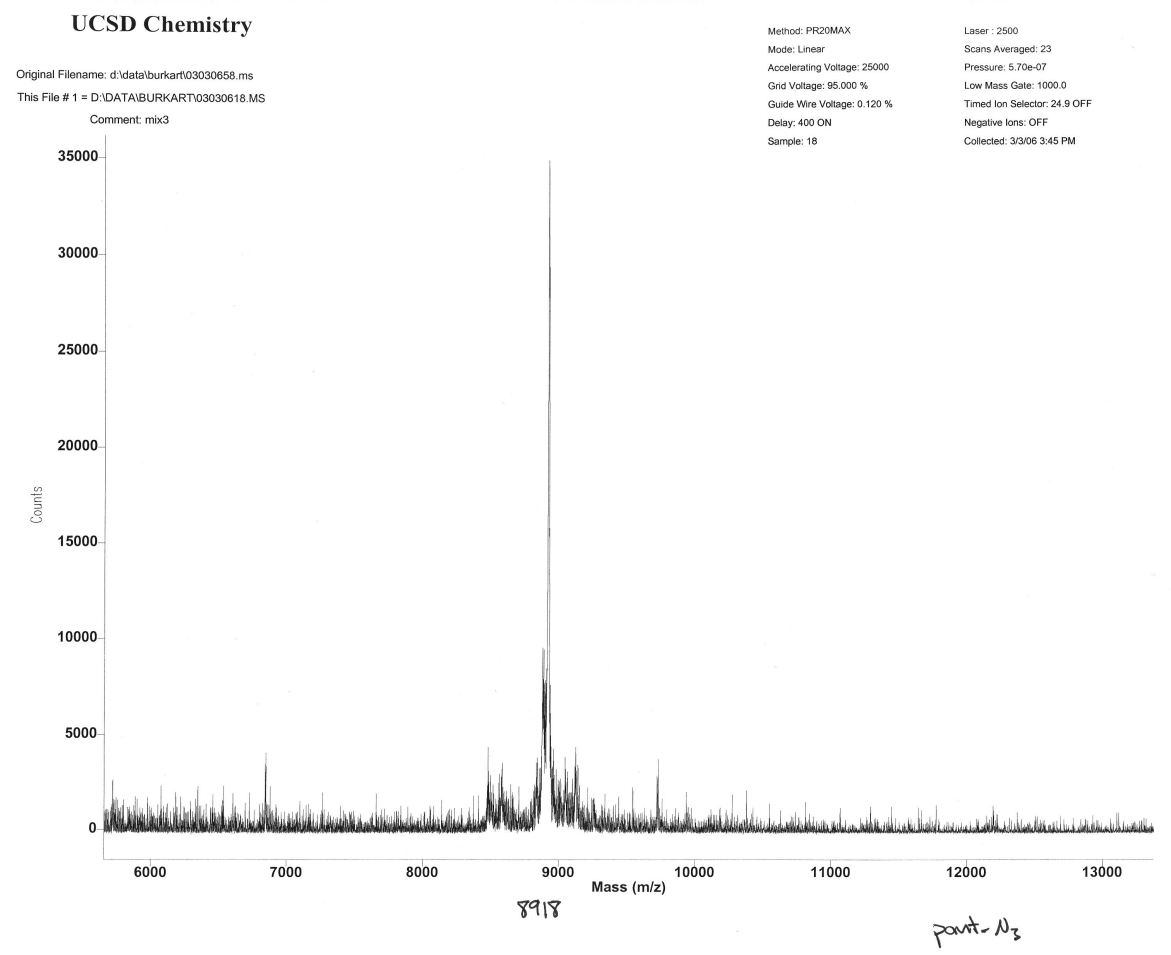


Compound $\mathbf{3}$ In vitro after click reaction with $\mathbf{1 0}$
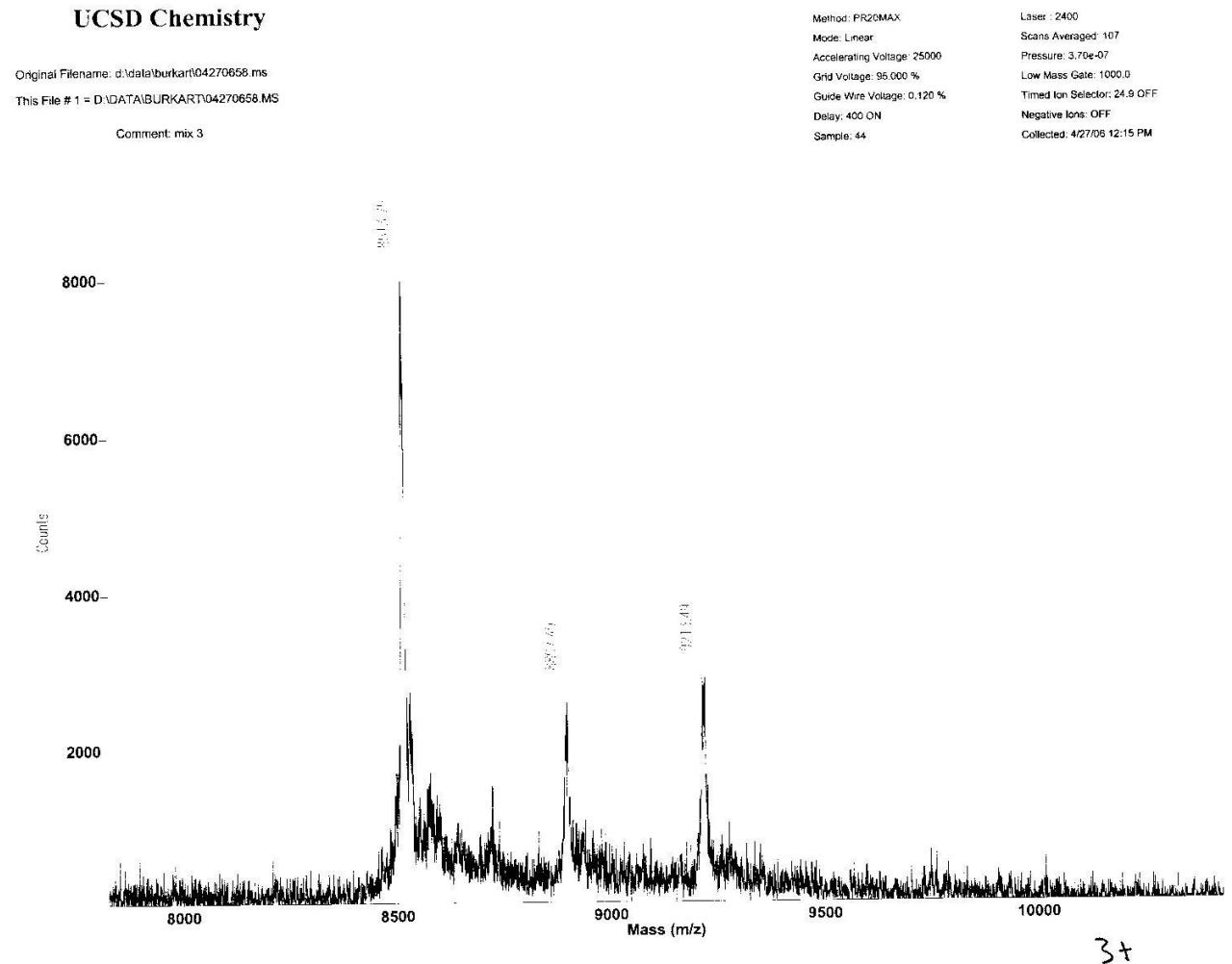

Compound 3 In vivo

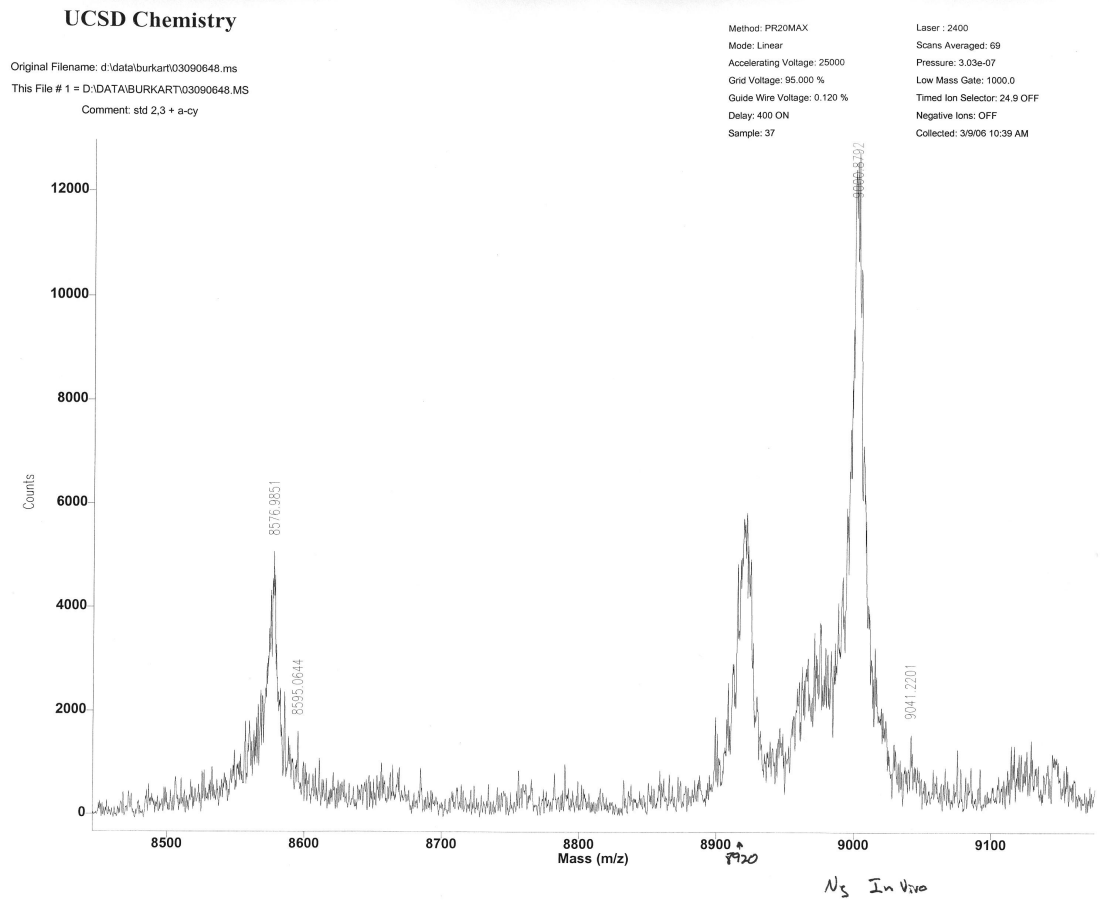


Compound $\mathbf{3}$ In vivo after click reaction with $\mathbf{1 0}$

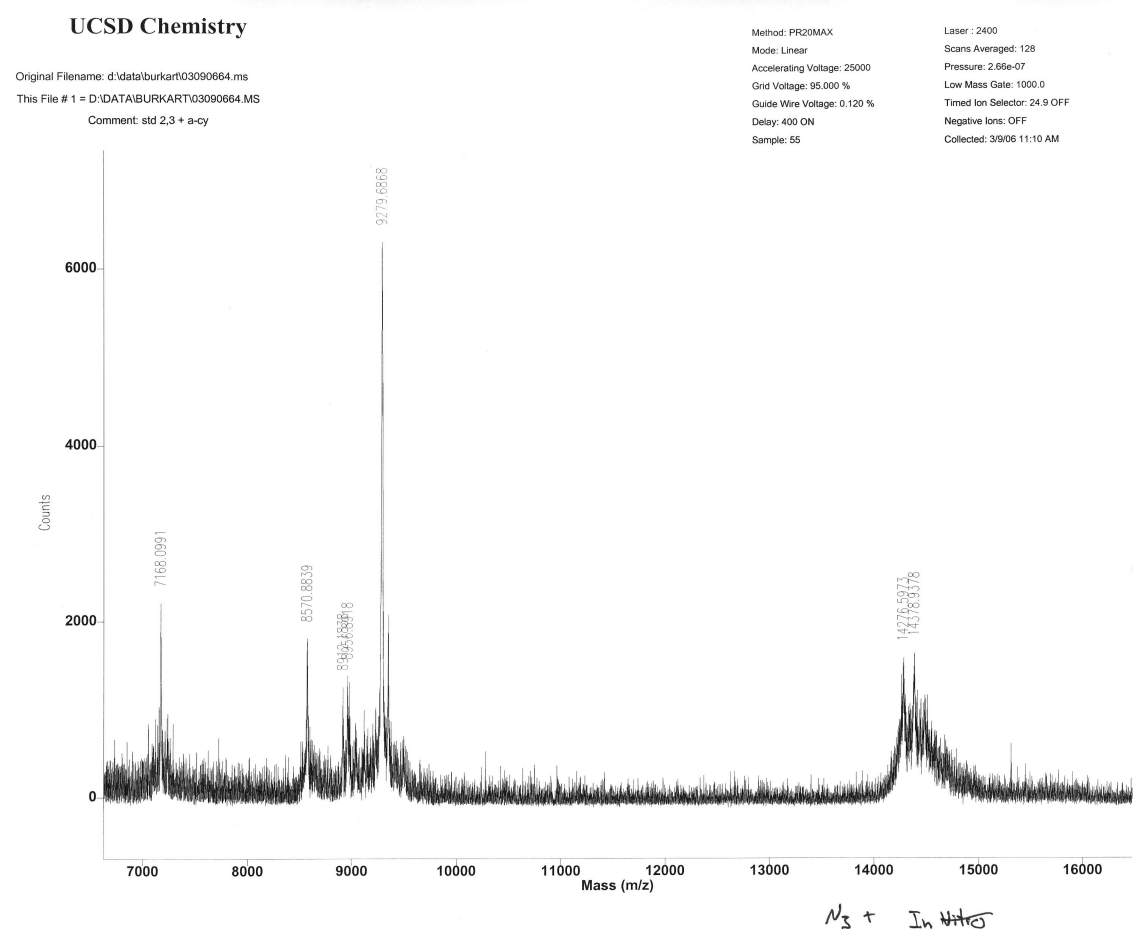

Compound 4 In vitro

UCSD Chemistry

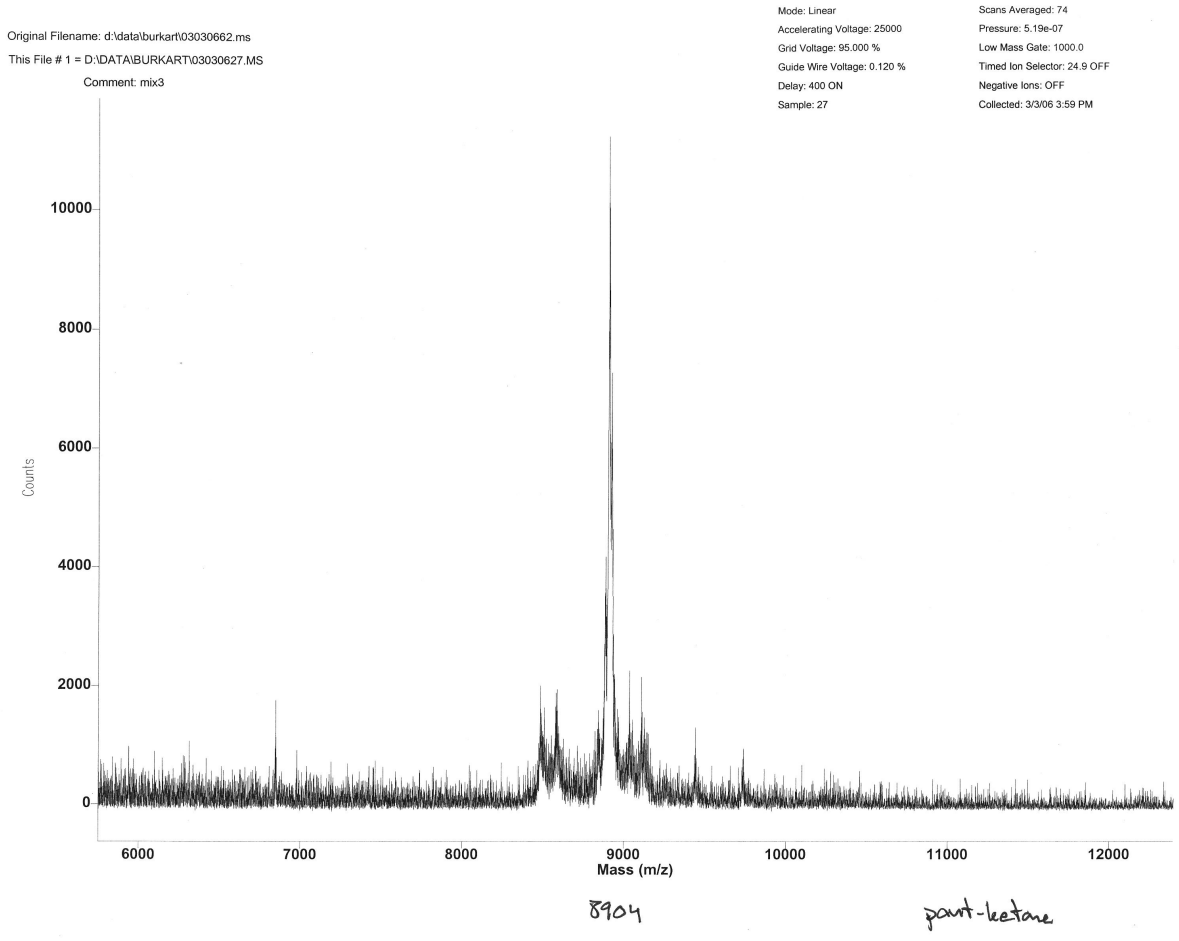


Compound 4 In vitro after reaction with 9

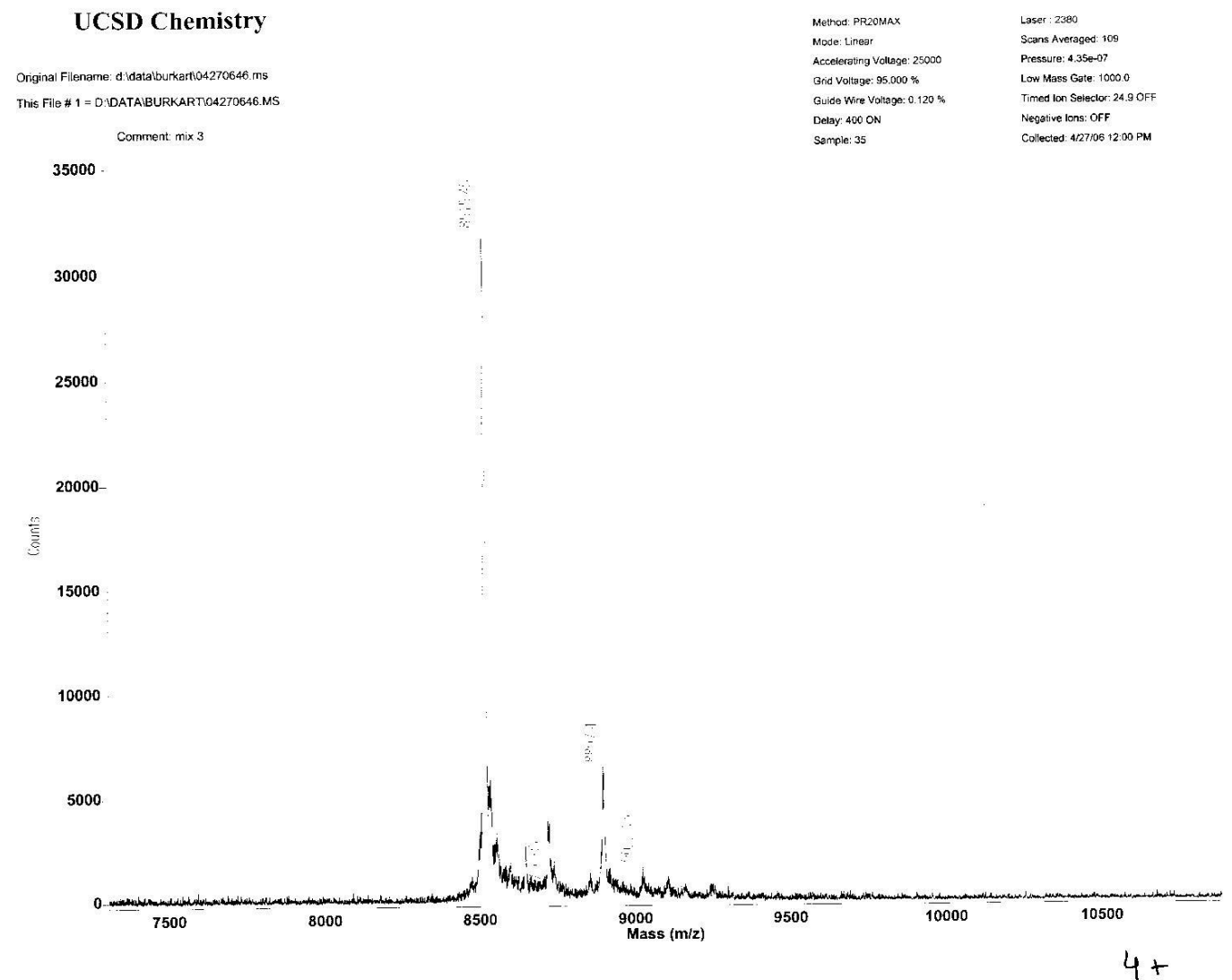

Compound 4 In vivo

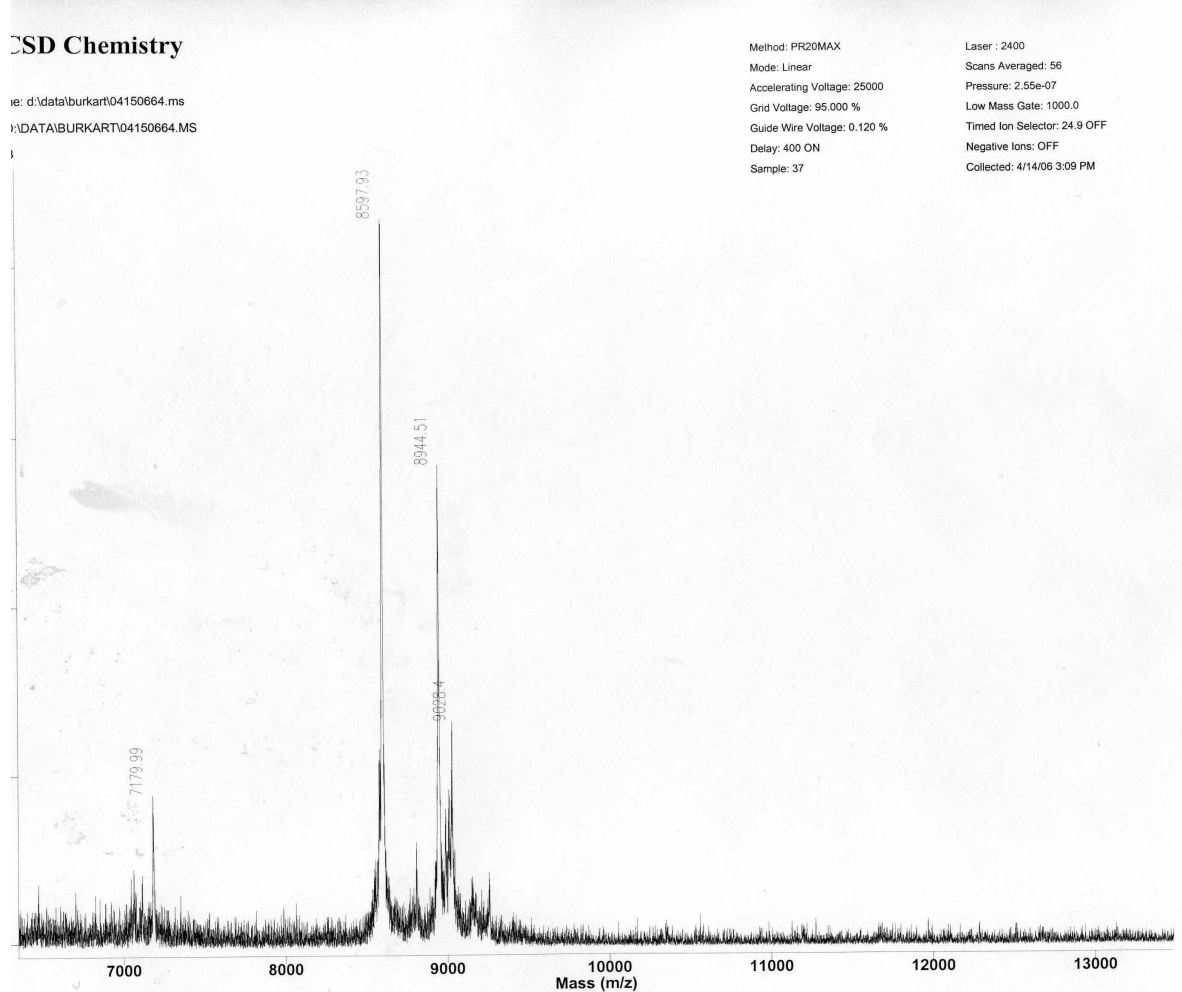


Compound 4 In vivo after reaction with 9

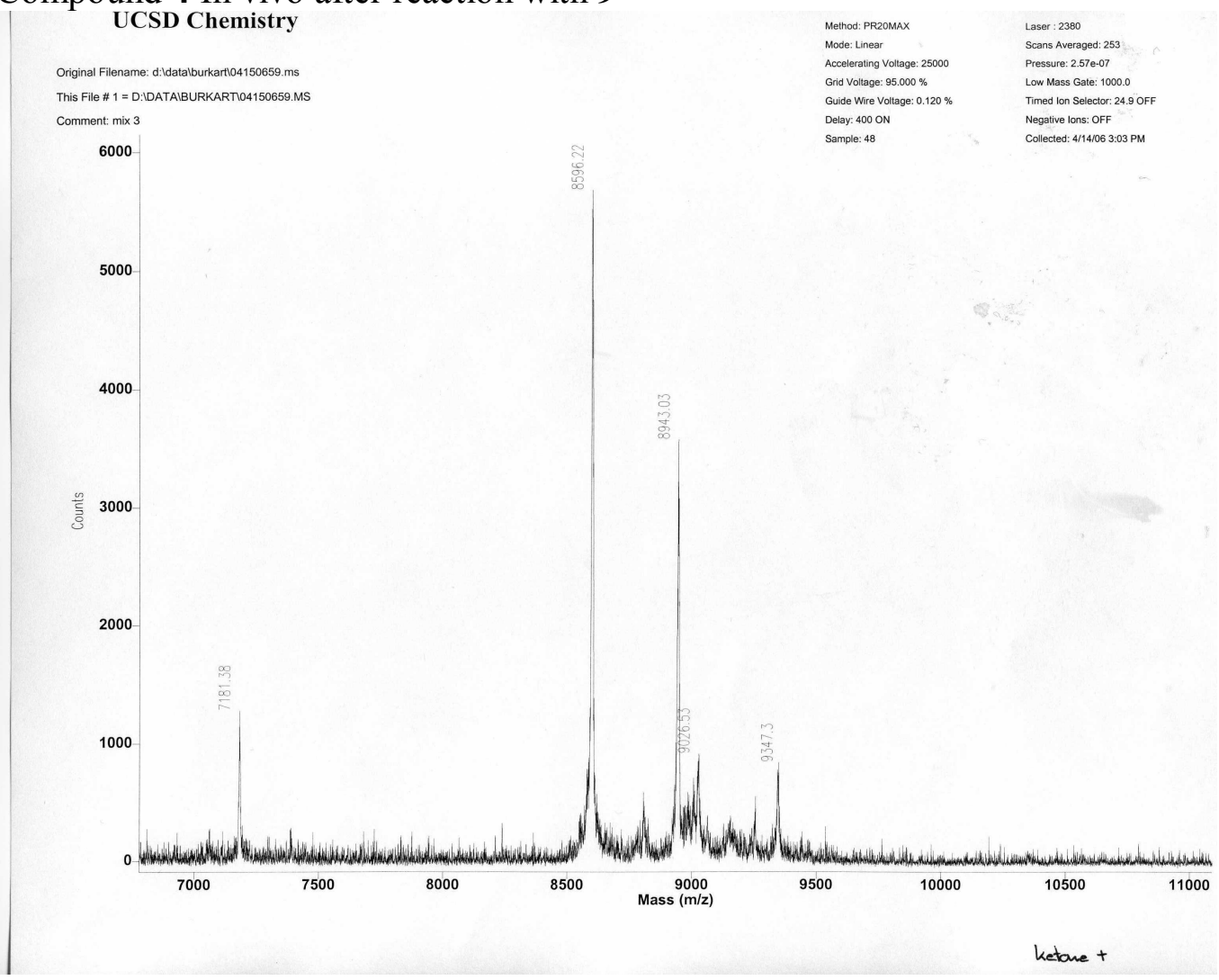

Compound 5 In vitro

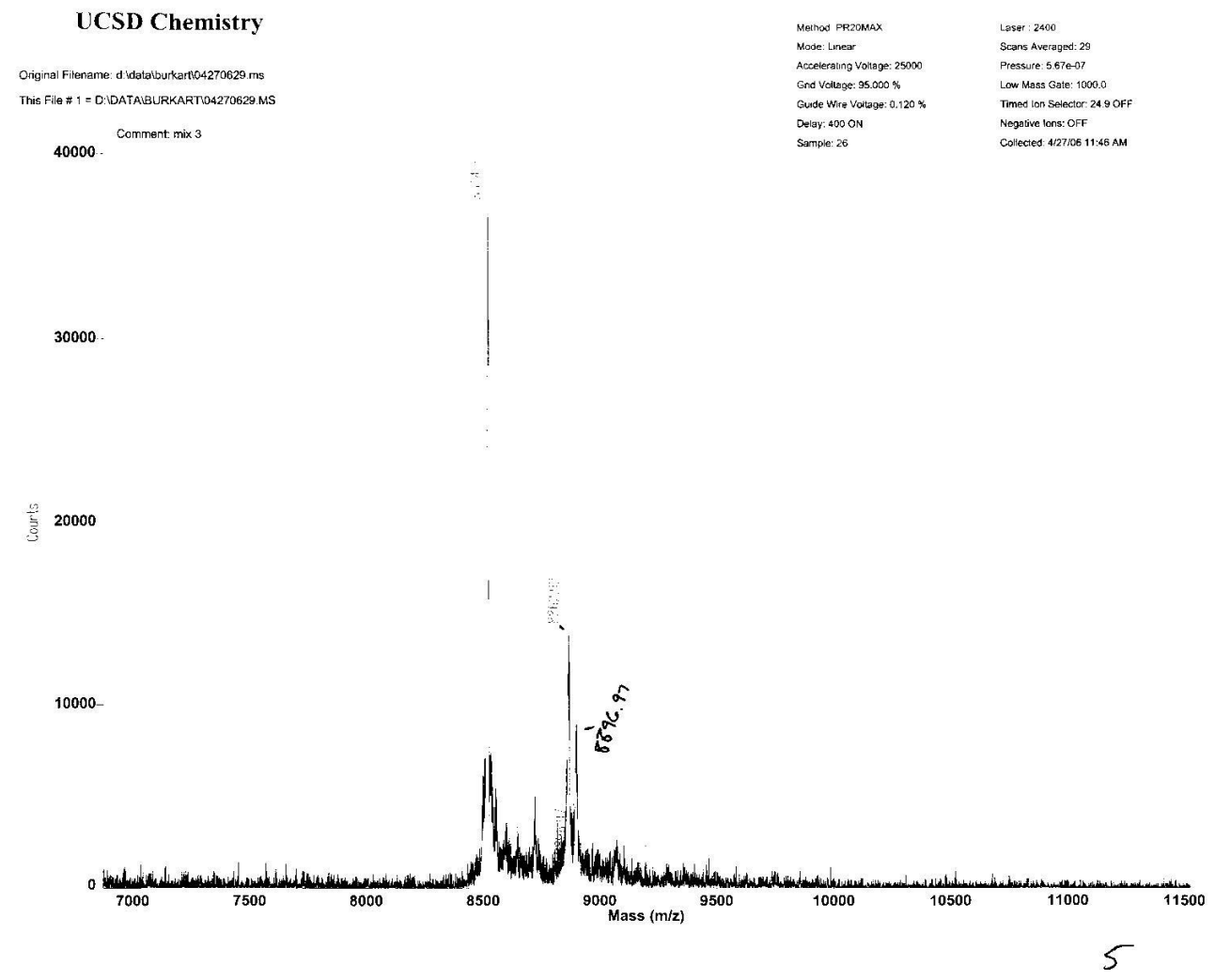


Compound 5 In vitro after click reaction with $\mathbf{1 0}$

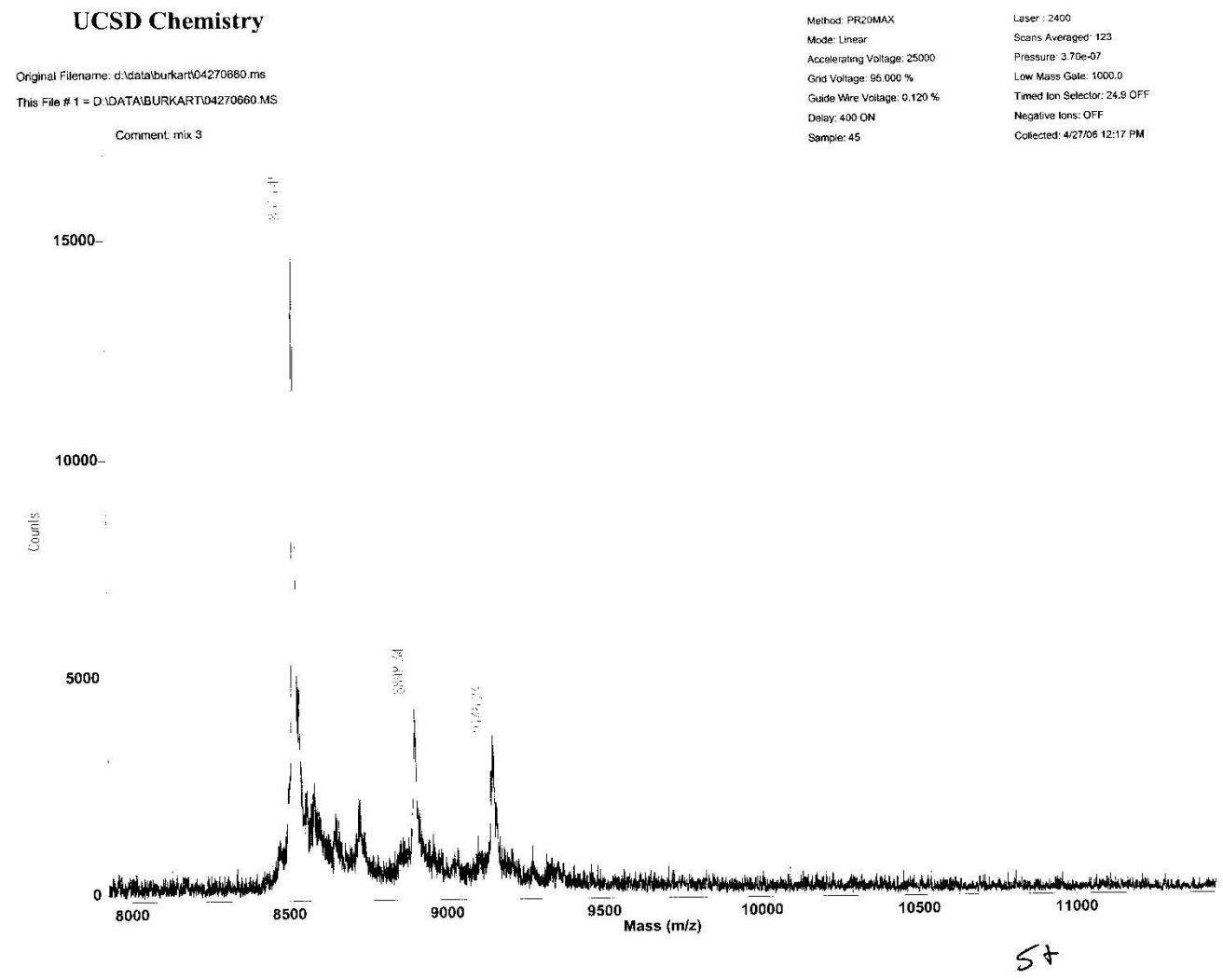

Compound 5 In vivo

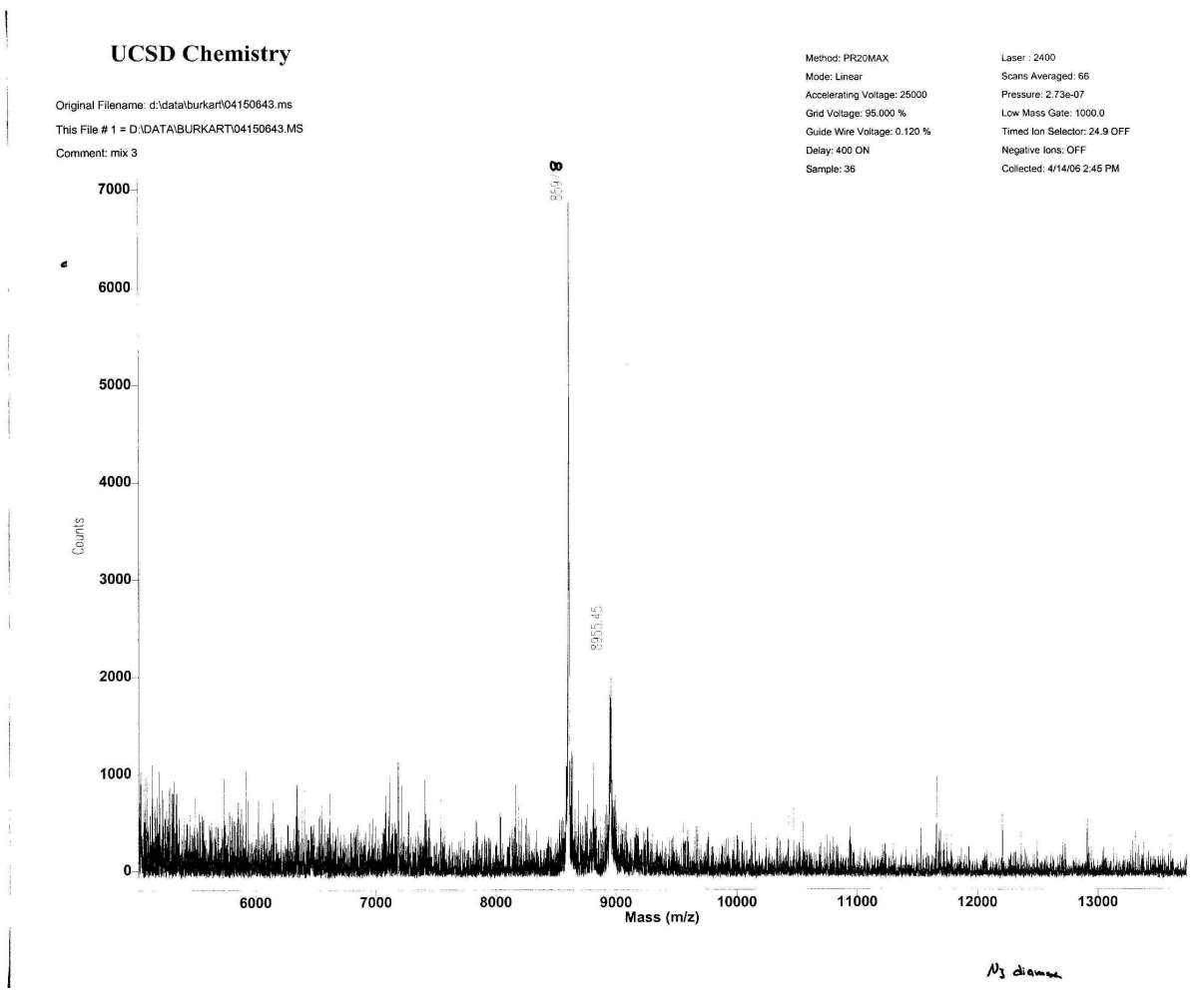


Compound 5 In vivo after click reaction with $\mathbf{1 0}$

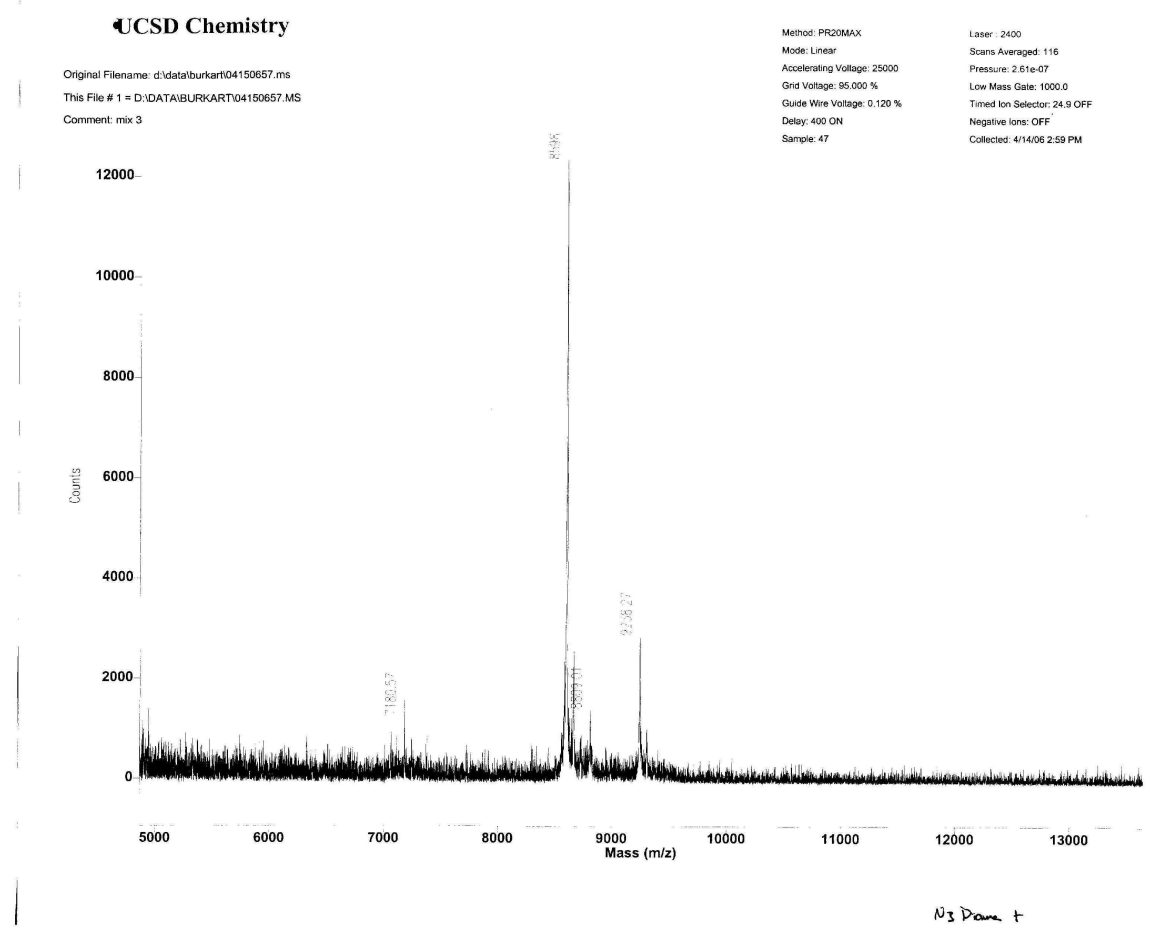

Compound 6 In vitro

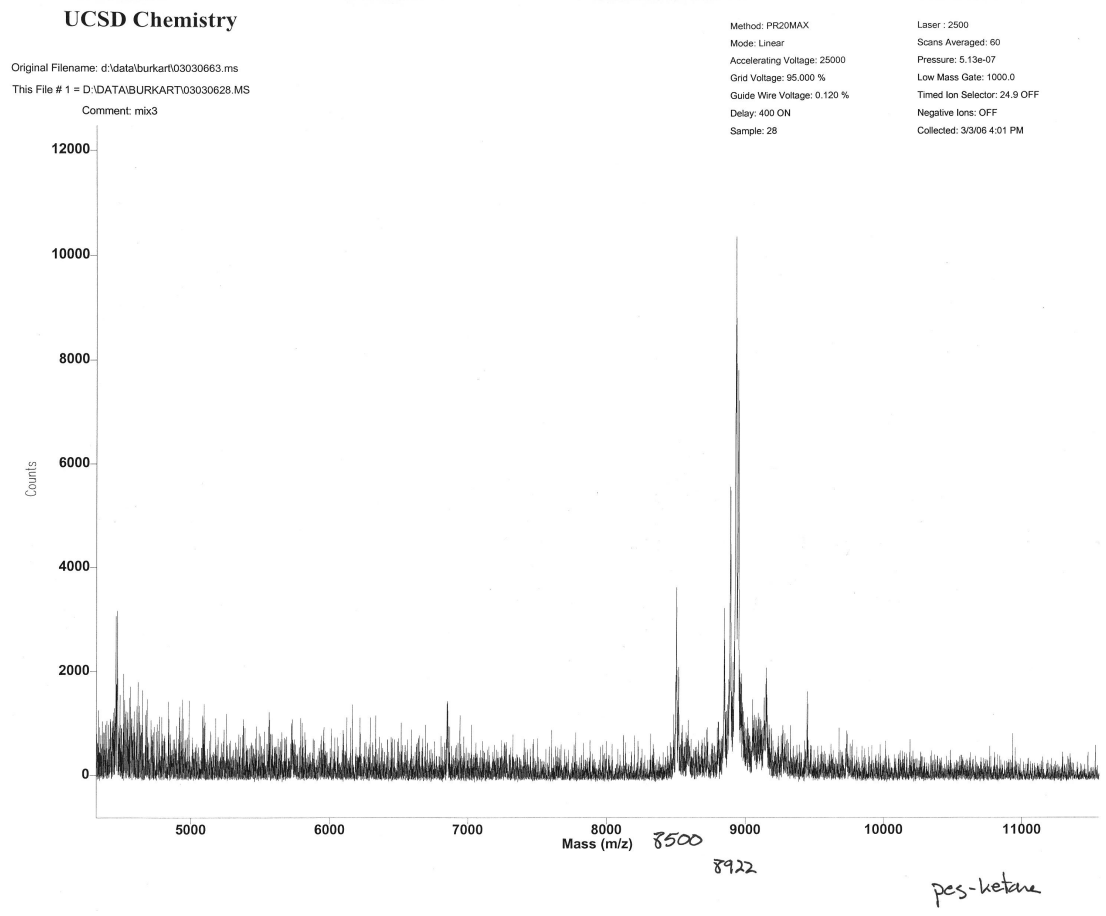




\section{Compound 6 In vivo}

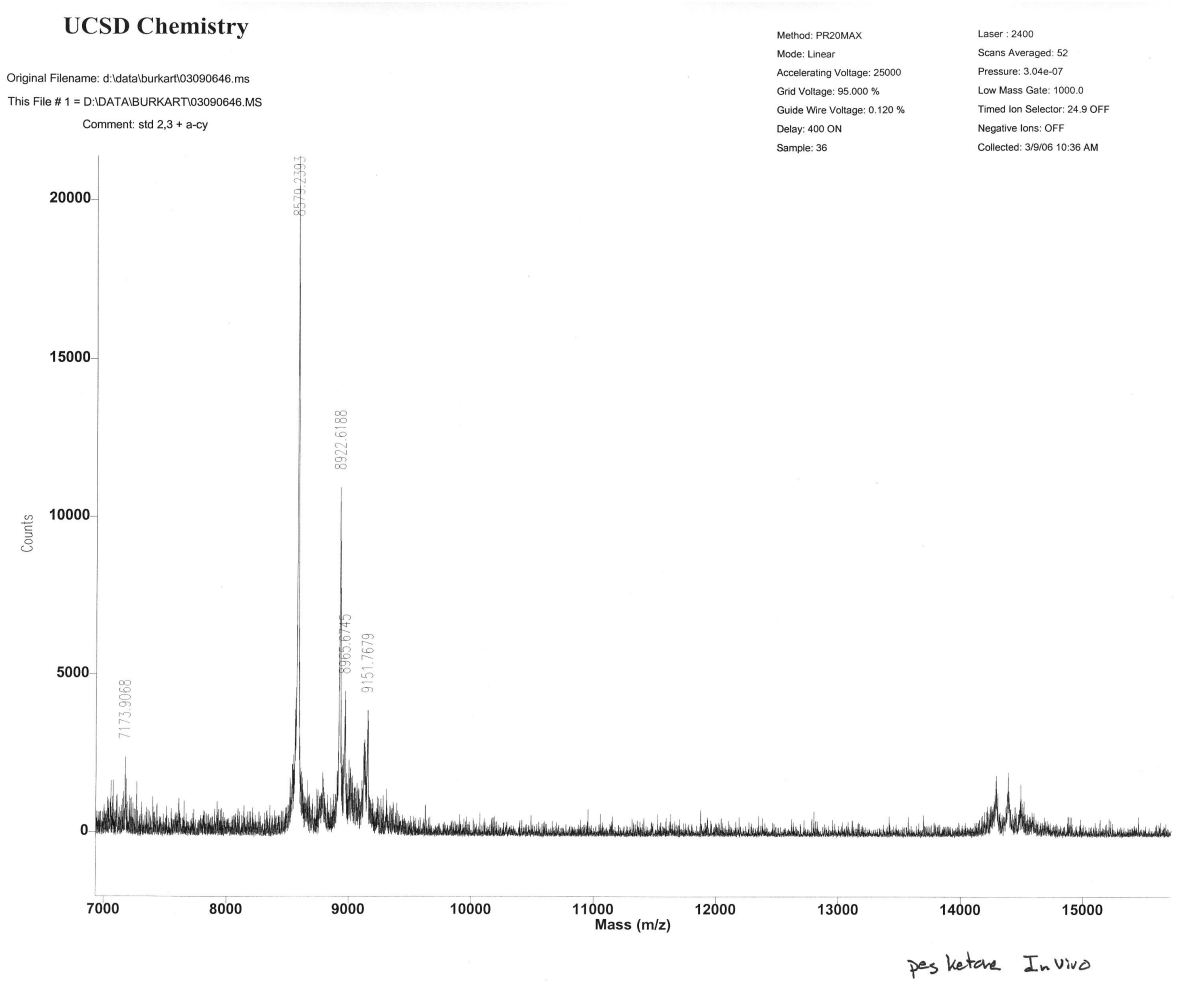

Compound 6 In vivo after reaction with 9

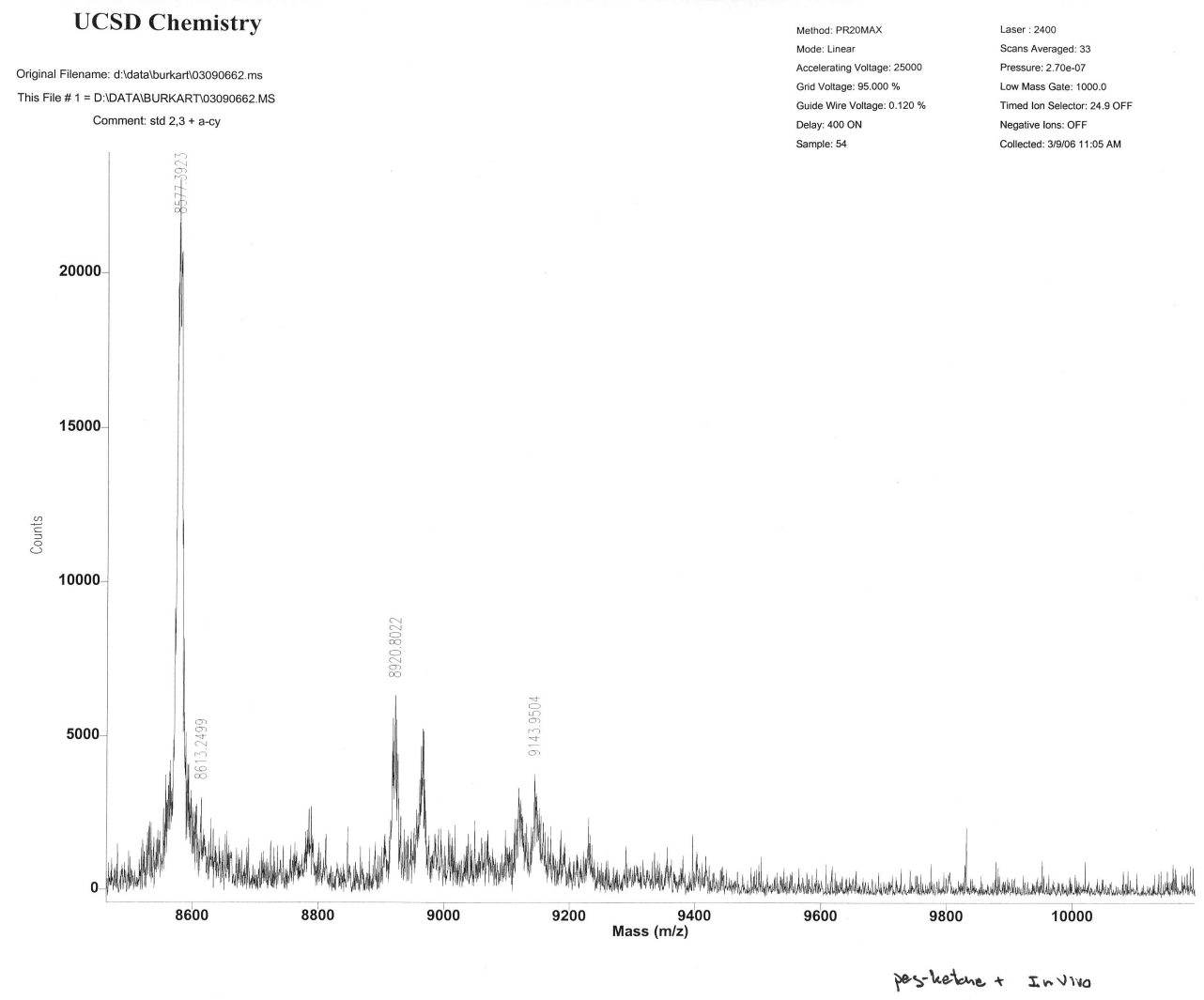


Compound 7 In vitro

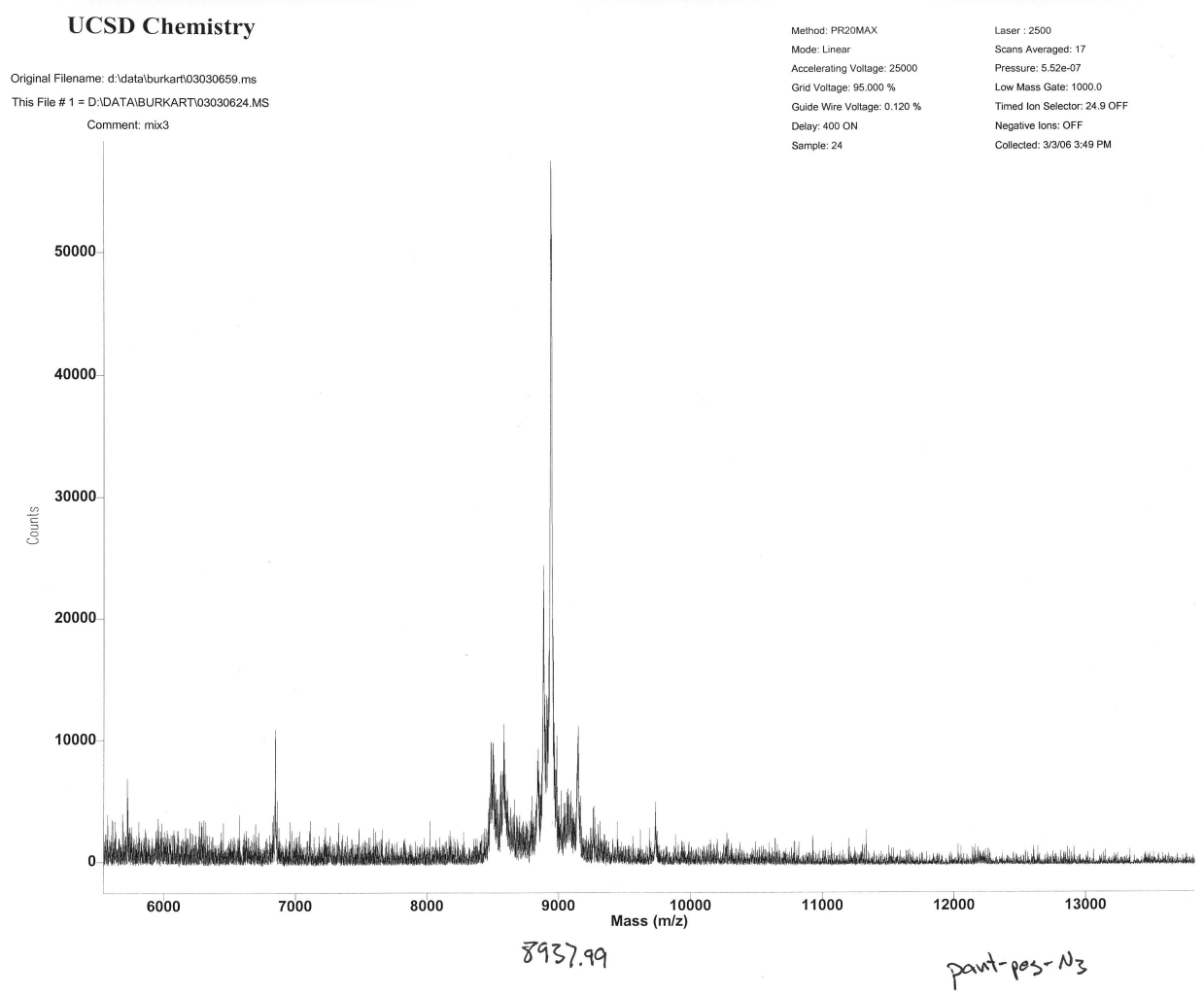

Compound 7 In vitro after click reaction with $\mathbf{1 0}$
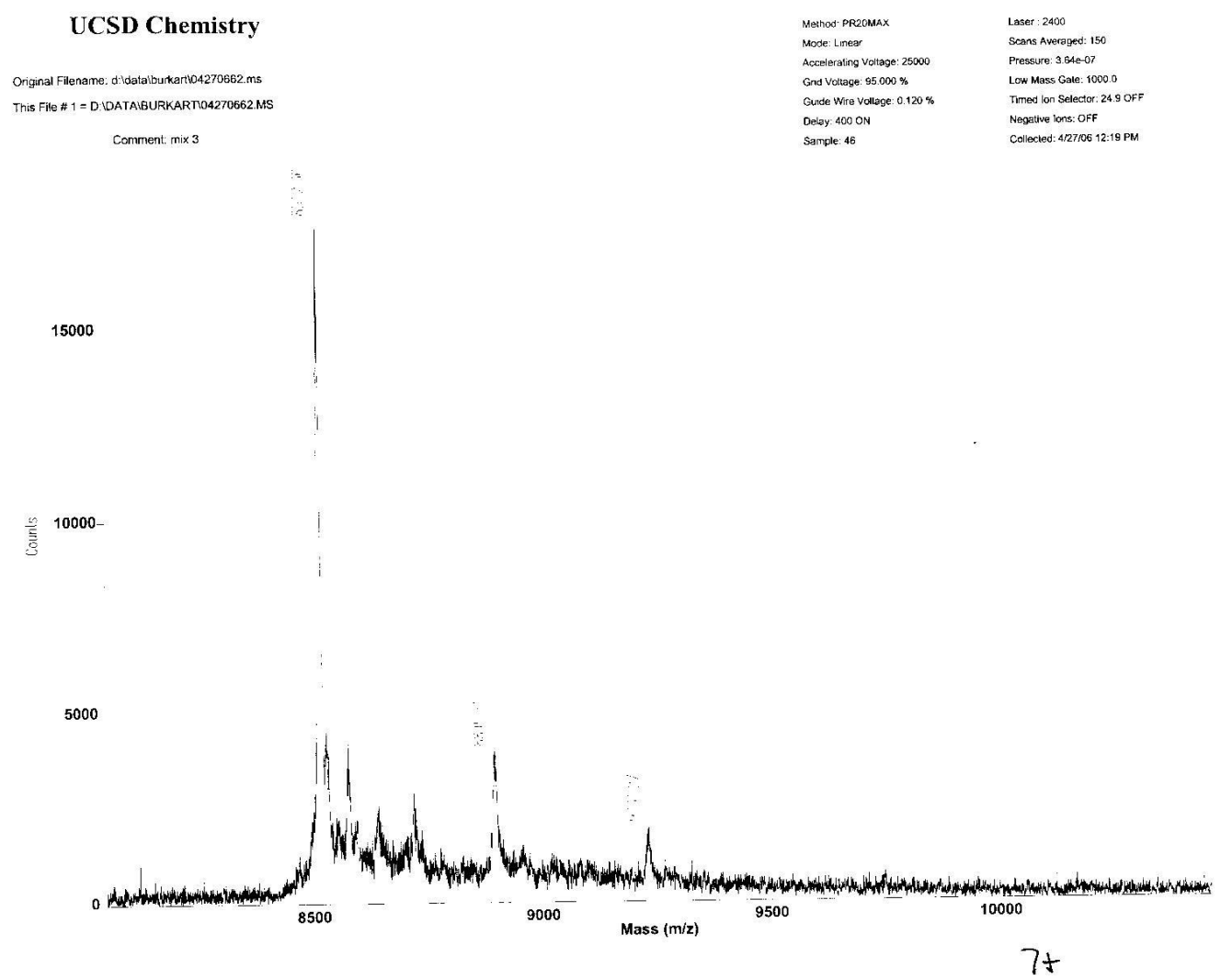


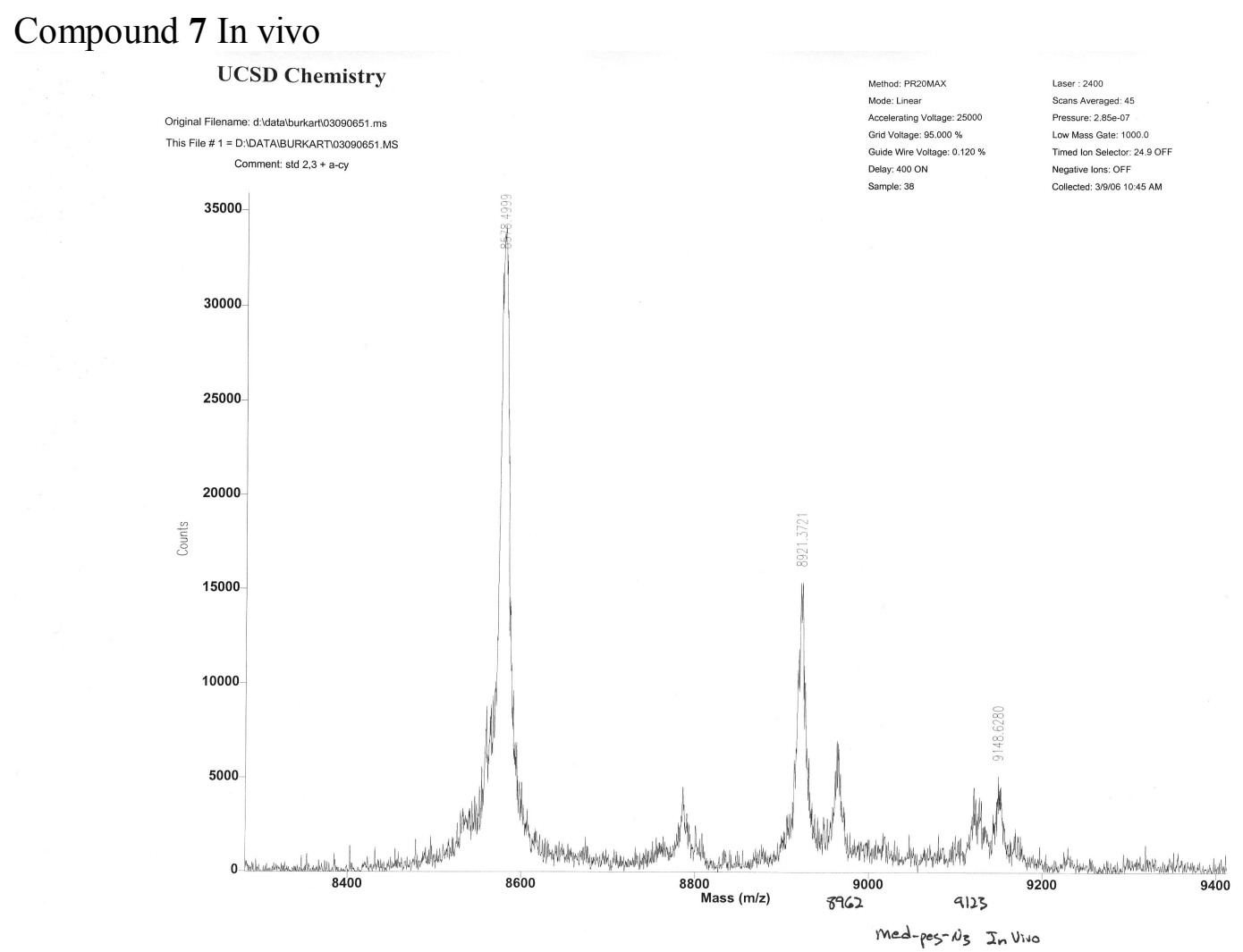

Compound 7 In vivo after click reaction with $\mathbf{1 0}$

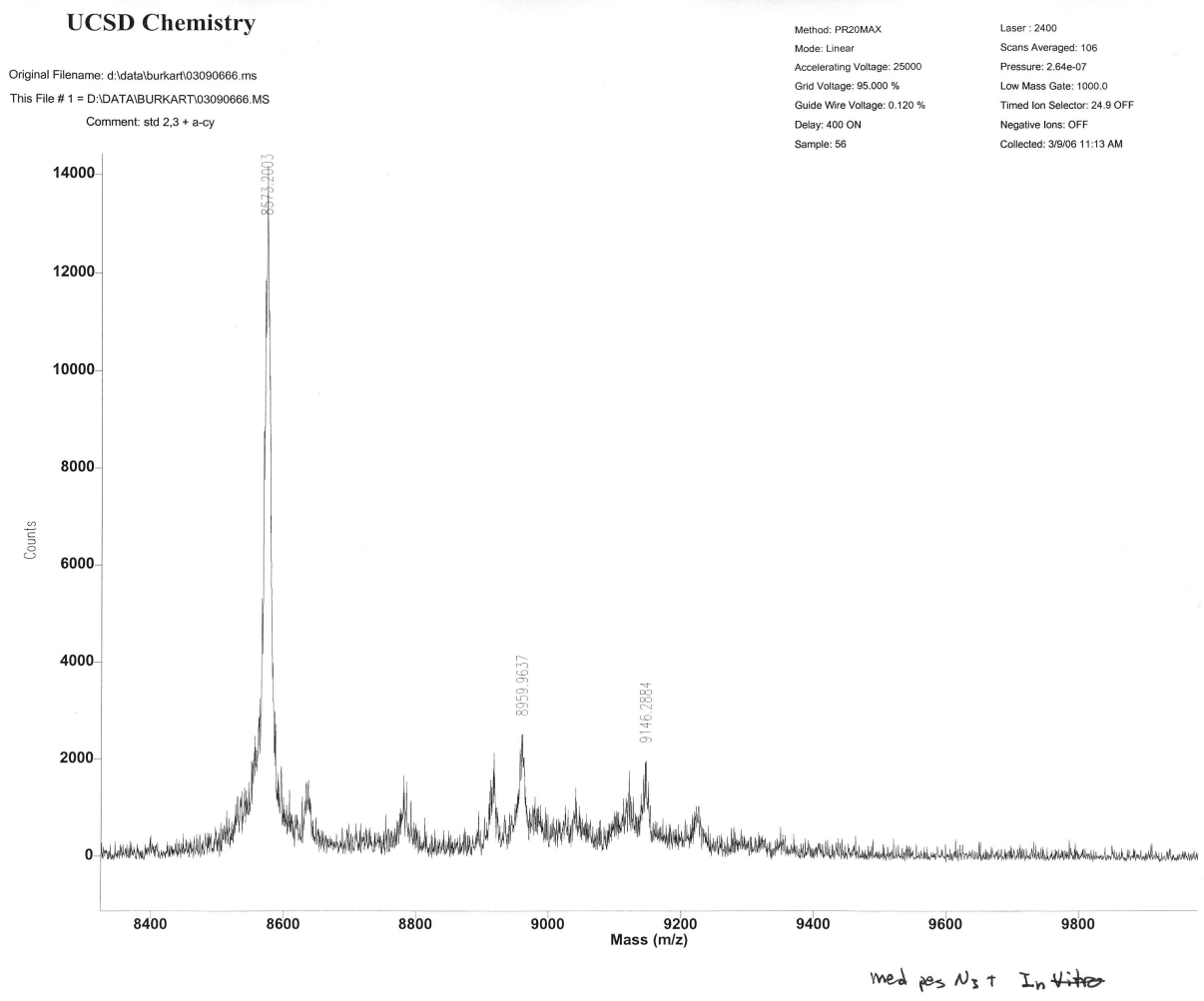


Compound 8 In vitro

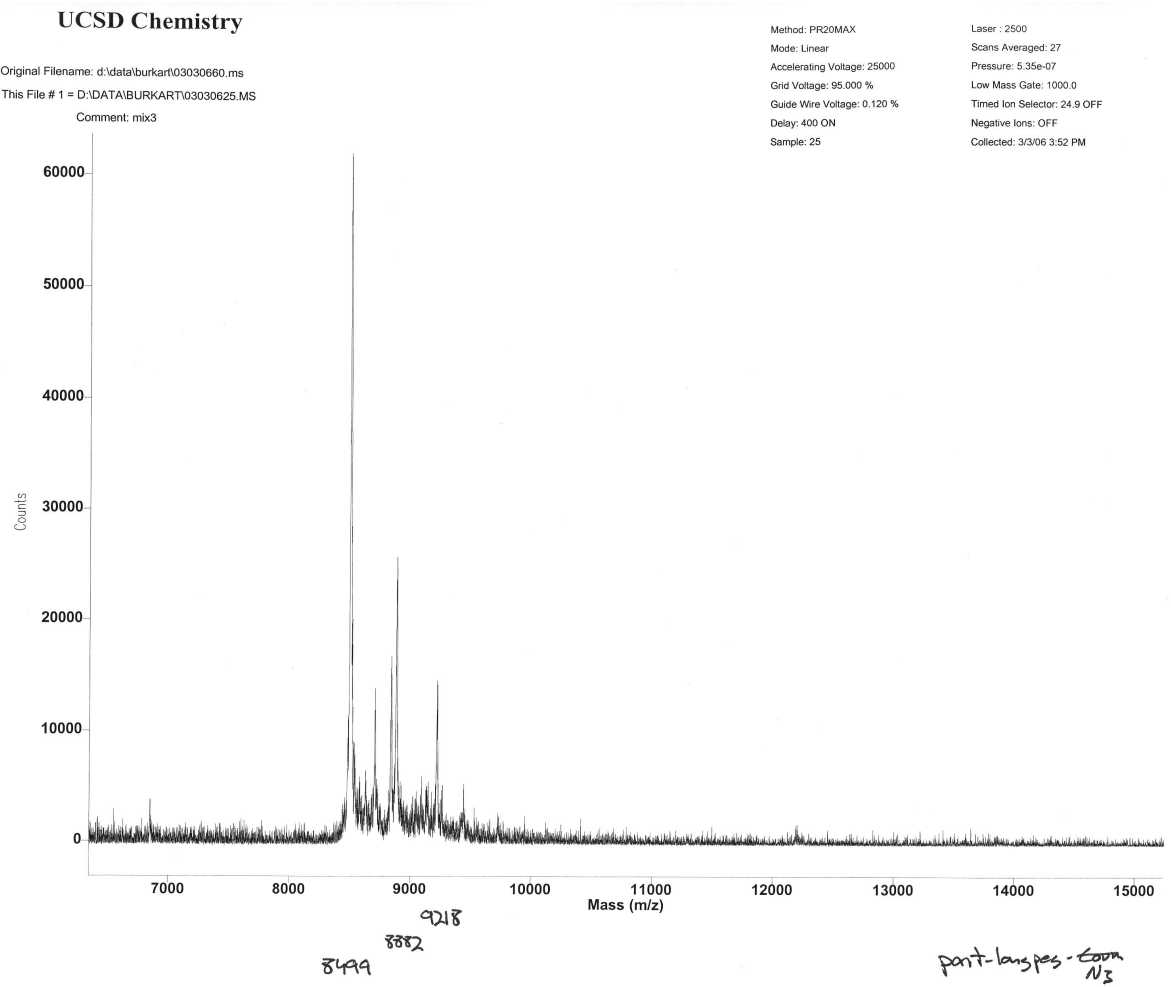

Compound 8 In vitro after click reaction with 10

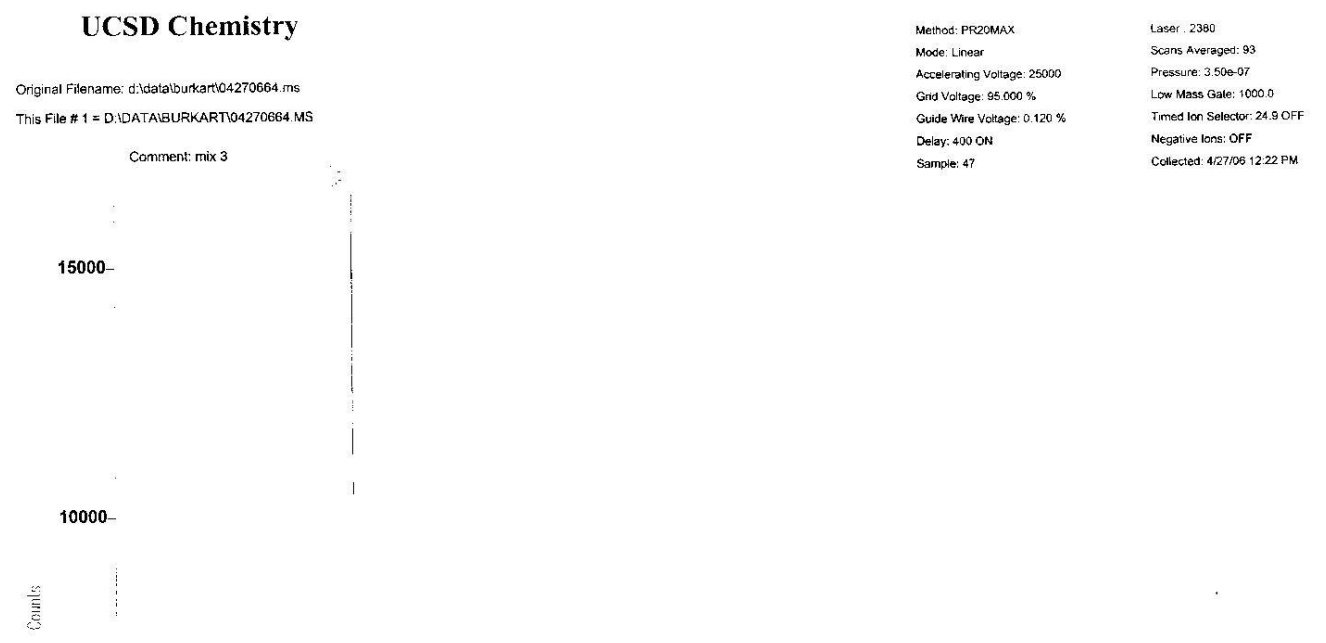

5000

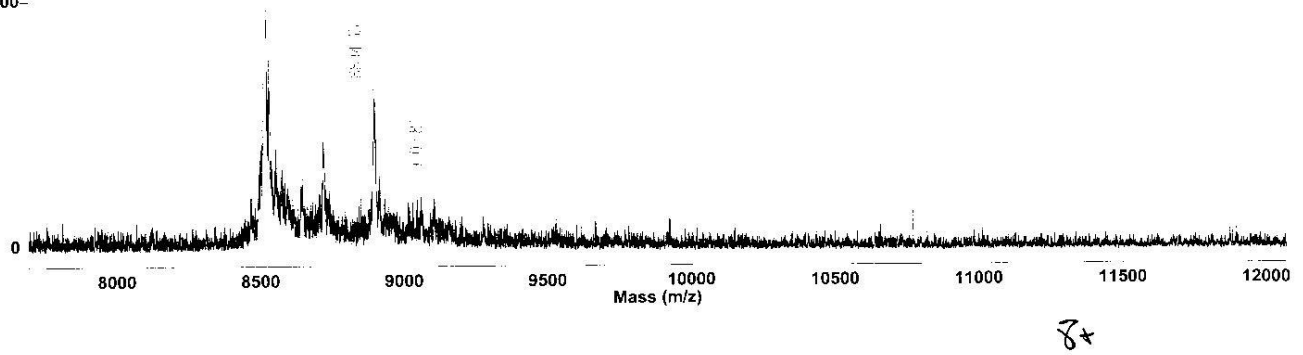




\section{Compound 8 In vivo}

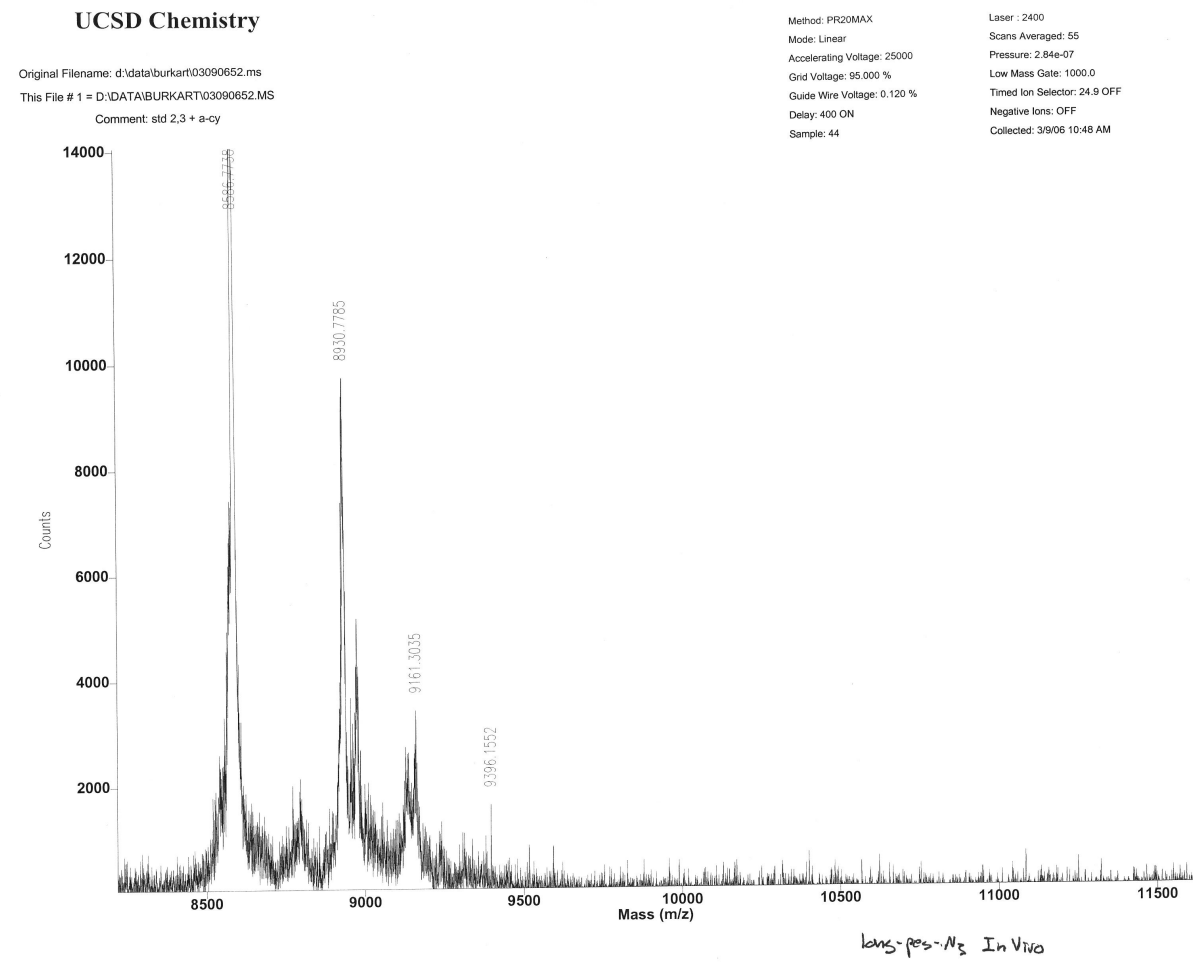

Compound 8 In vivo after click reaction with $\mathbf{1 0}$

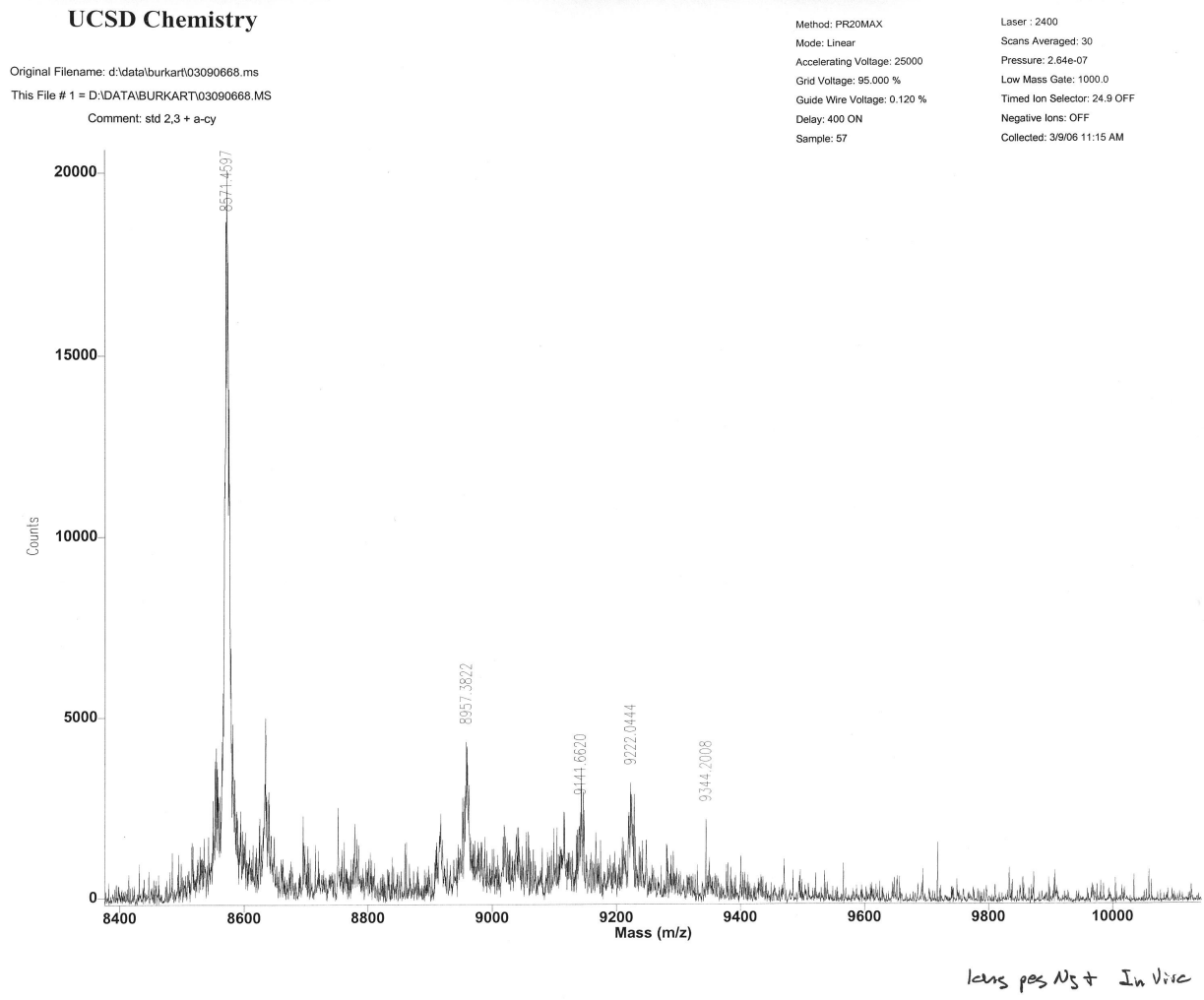




\section{Compound 12 In vitro}

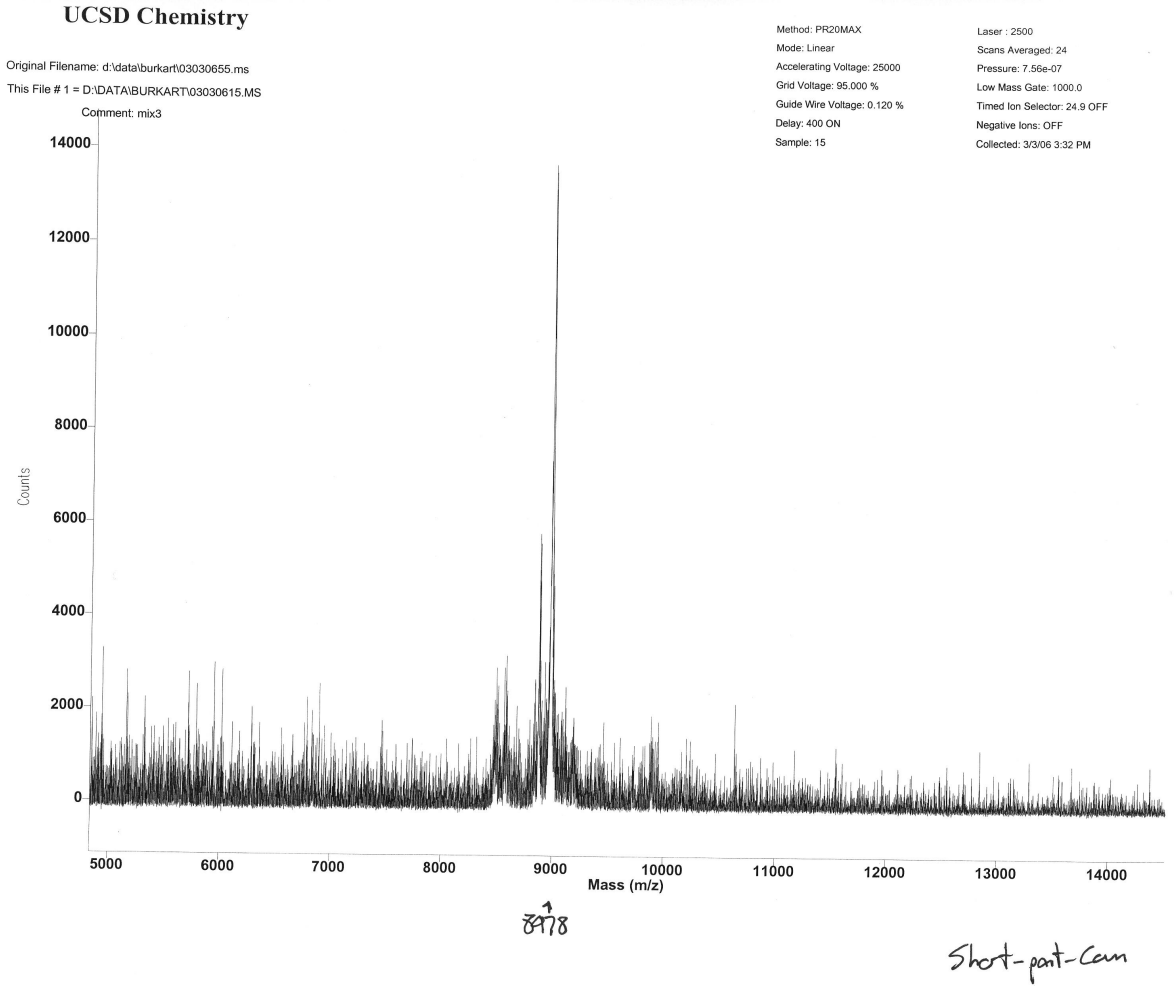

\section{Compound 12 In vivo}

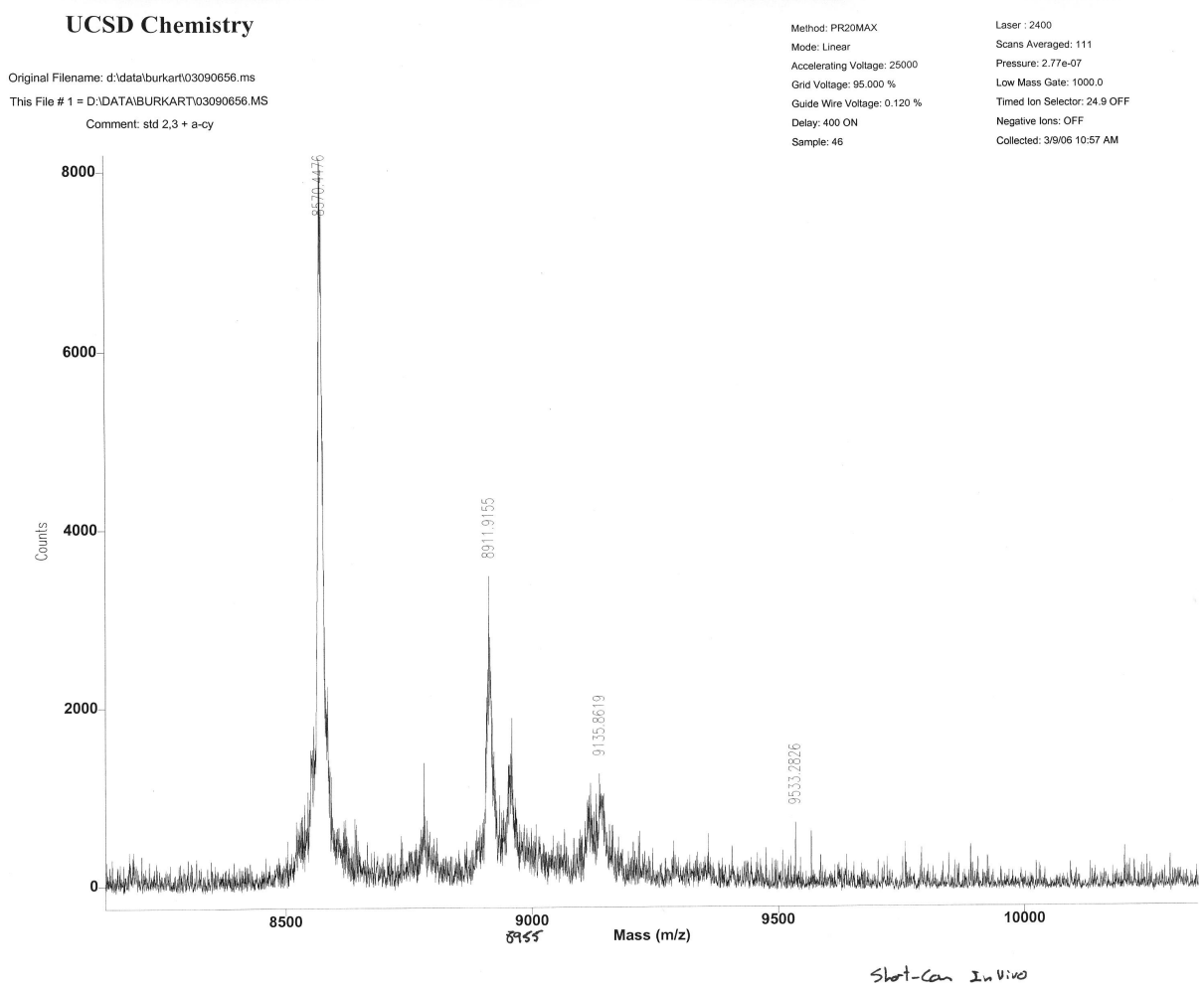


Compound 13 In vitro

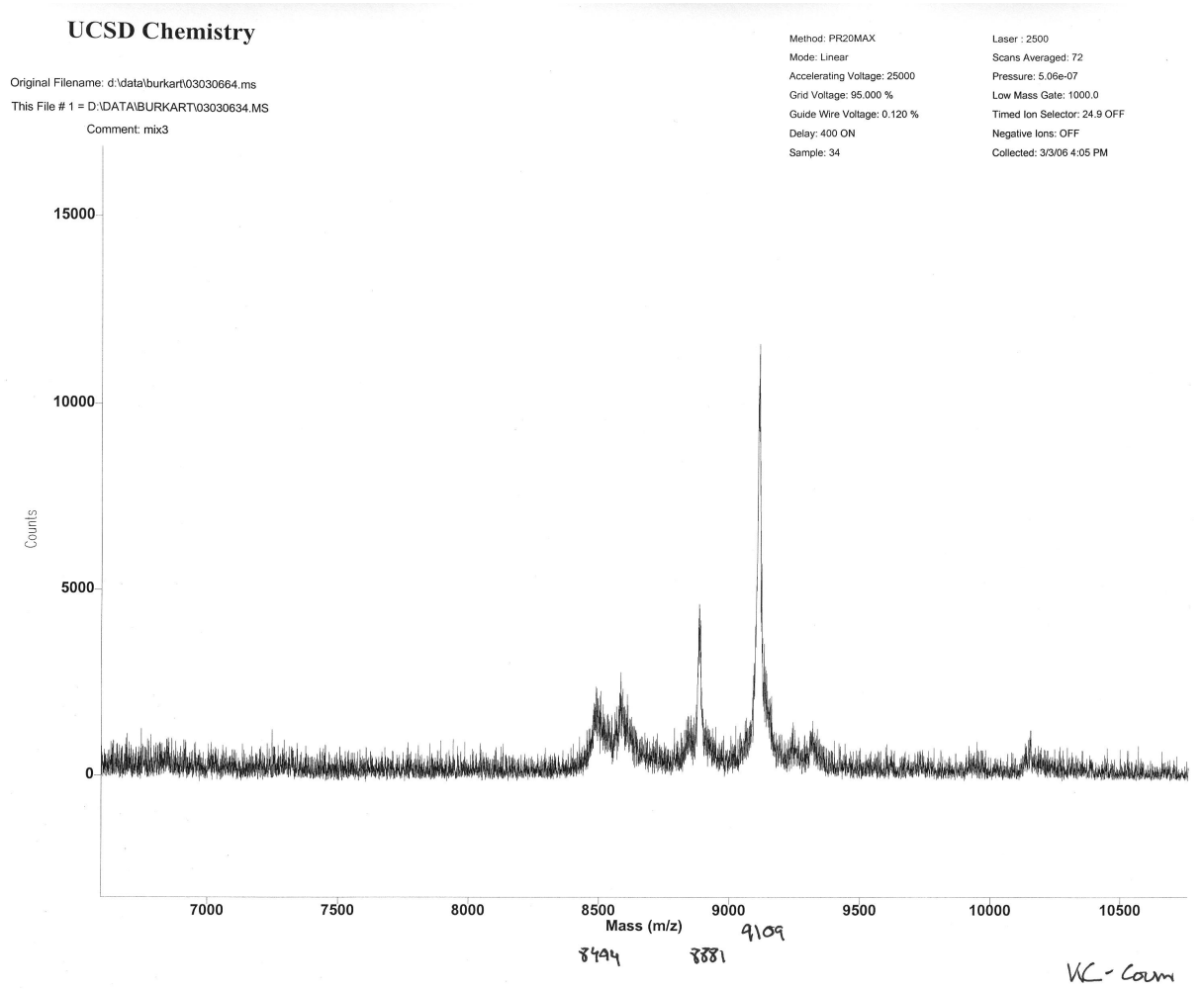

Compound 13 In vivo

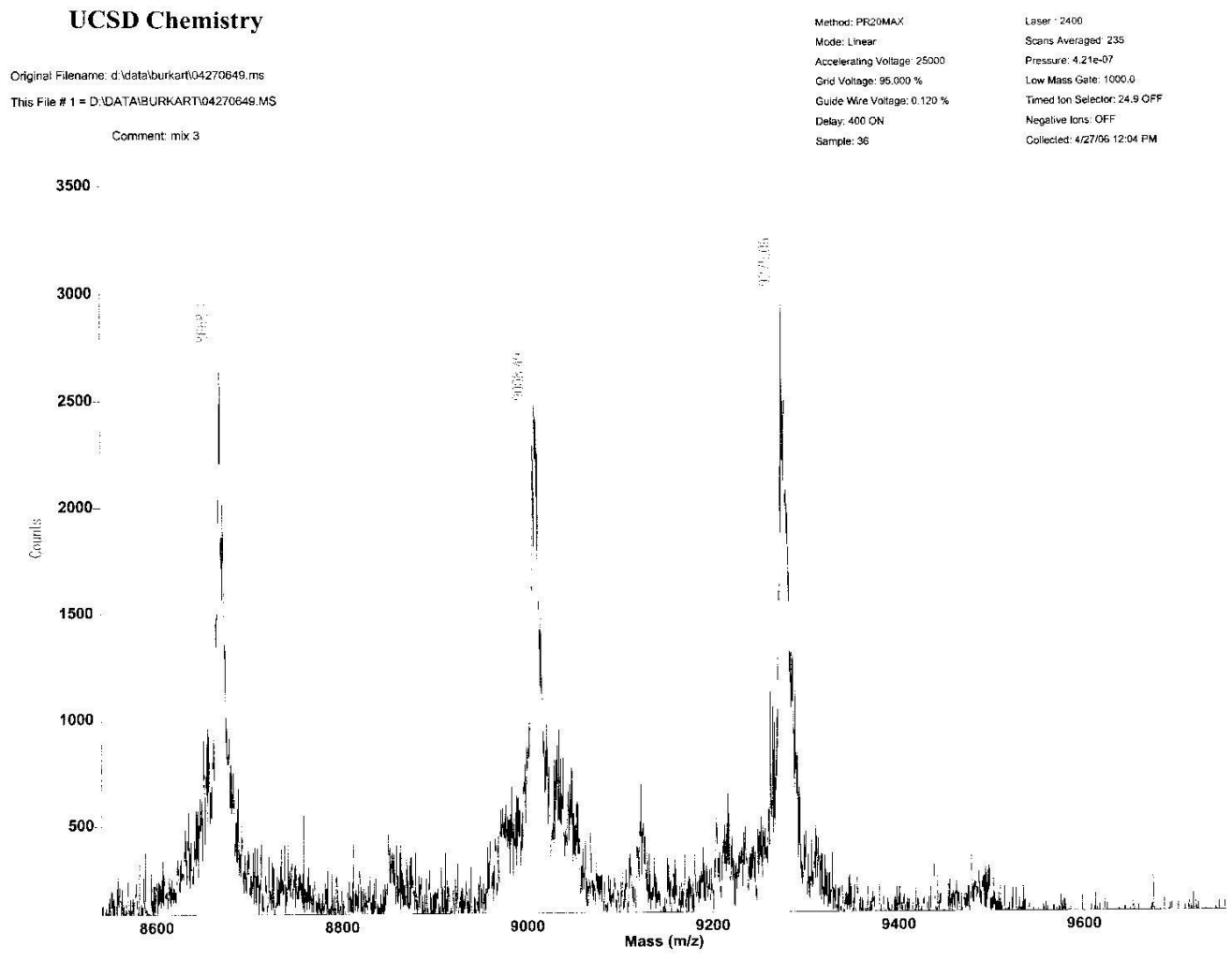

B W N 
Compound 14 In vitro

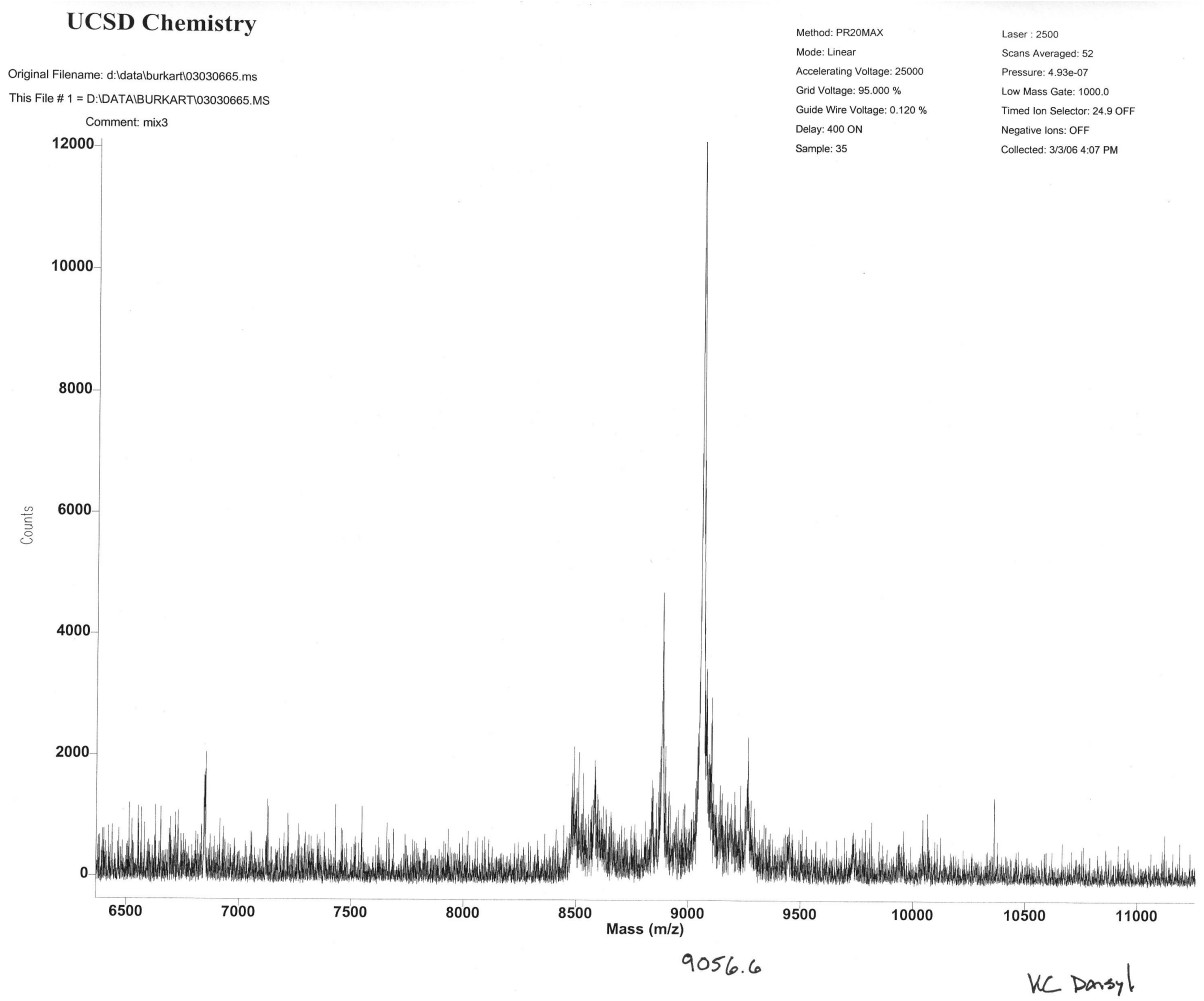

Compound 14 In vivo
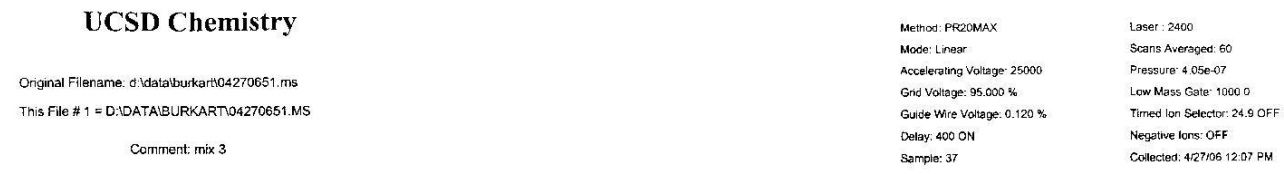

8000

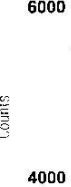

2000

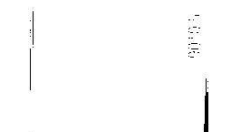

4000
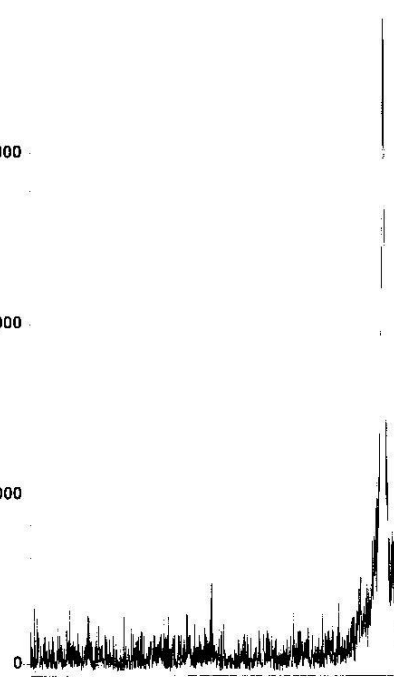

8500

9000

Mass $(m / z)$

9500 10000

14 Vivo 
Compound 15 In vitro

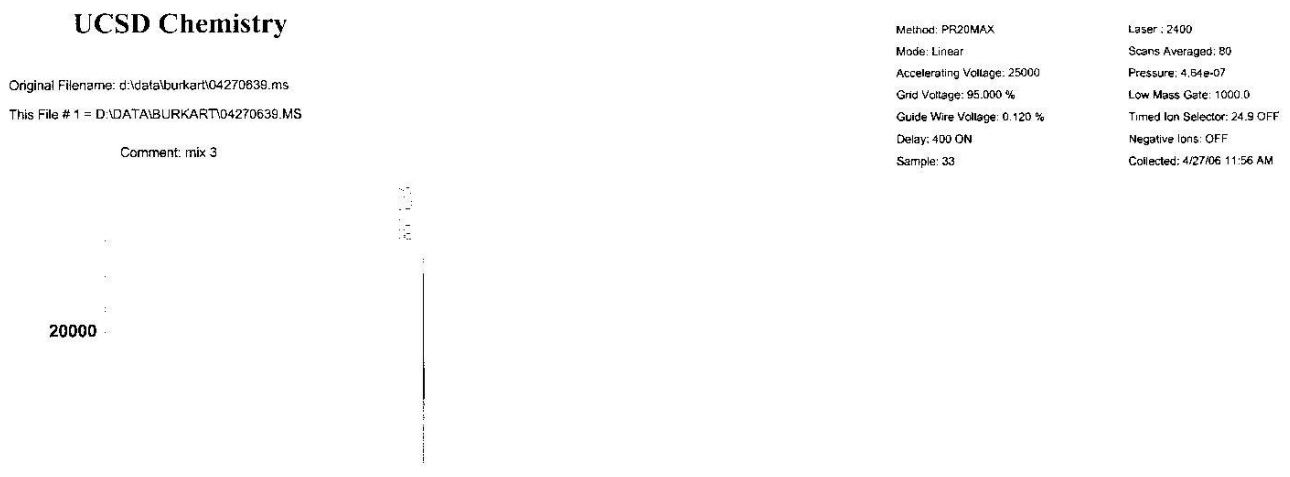

15000

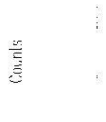

10000 -

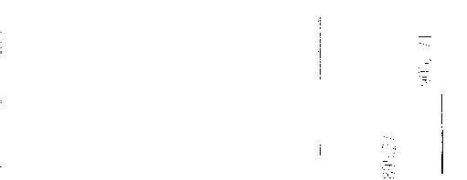

5000

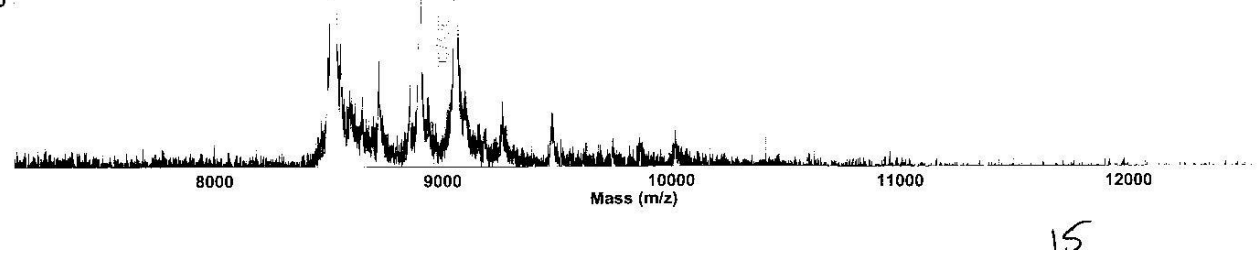

Compound 15 In vivo

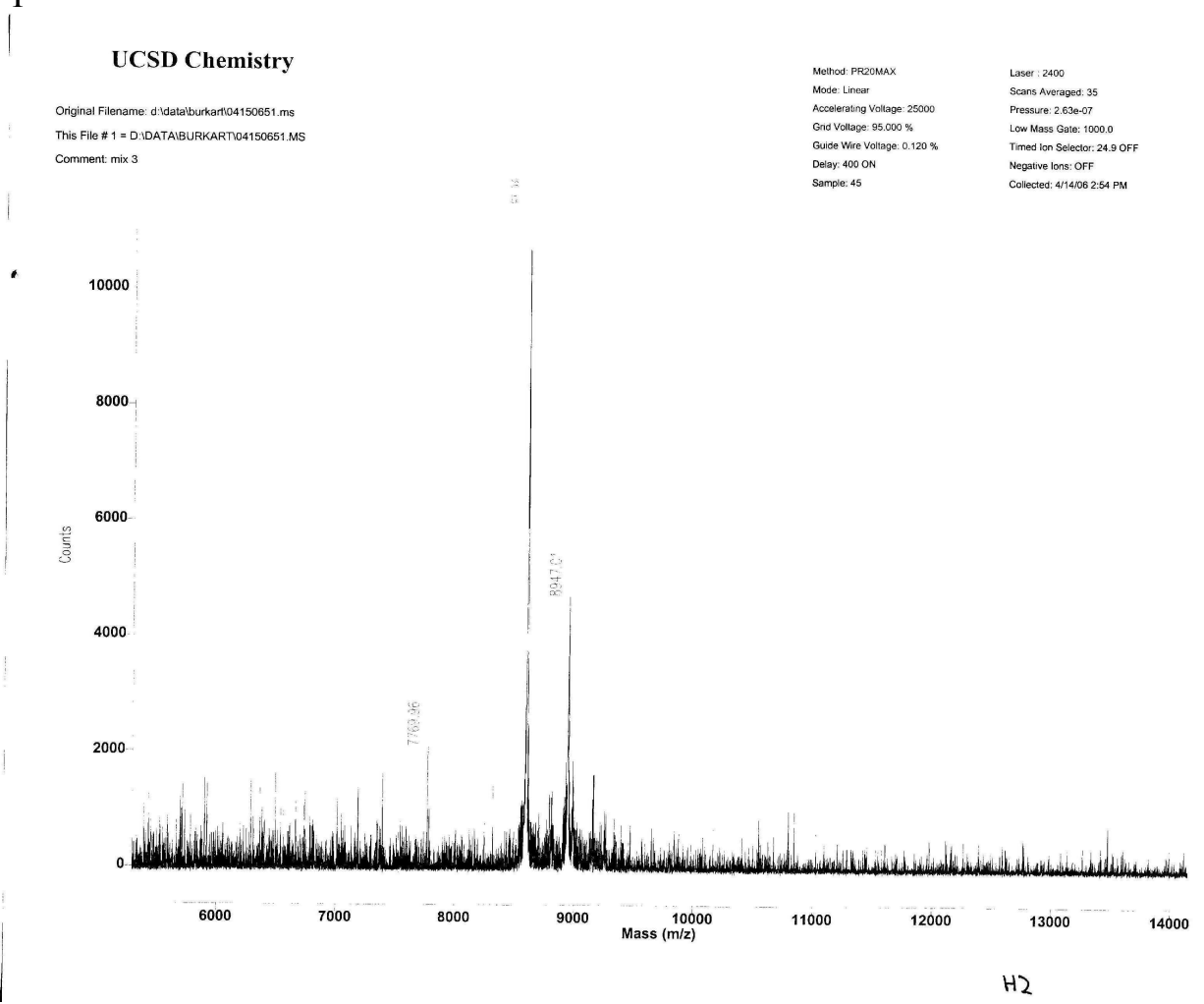


Compound 16 In vitro

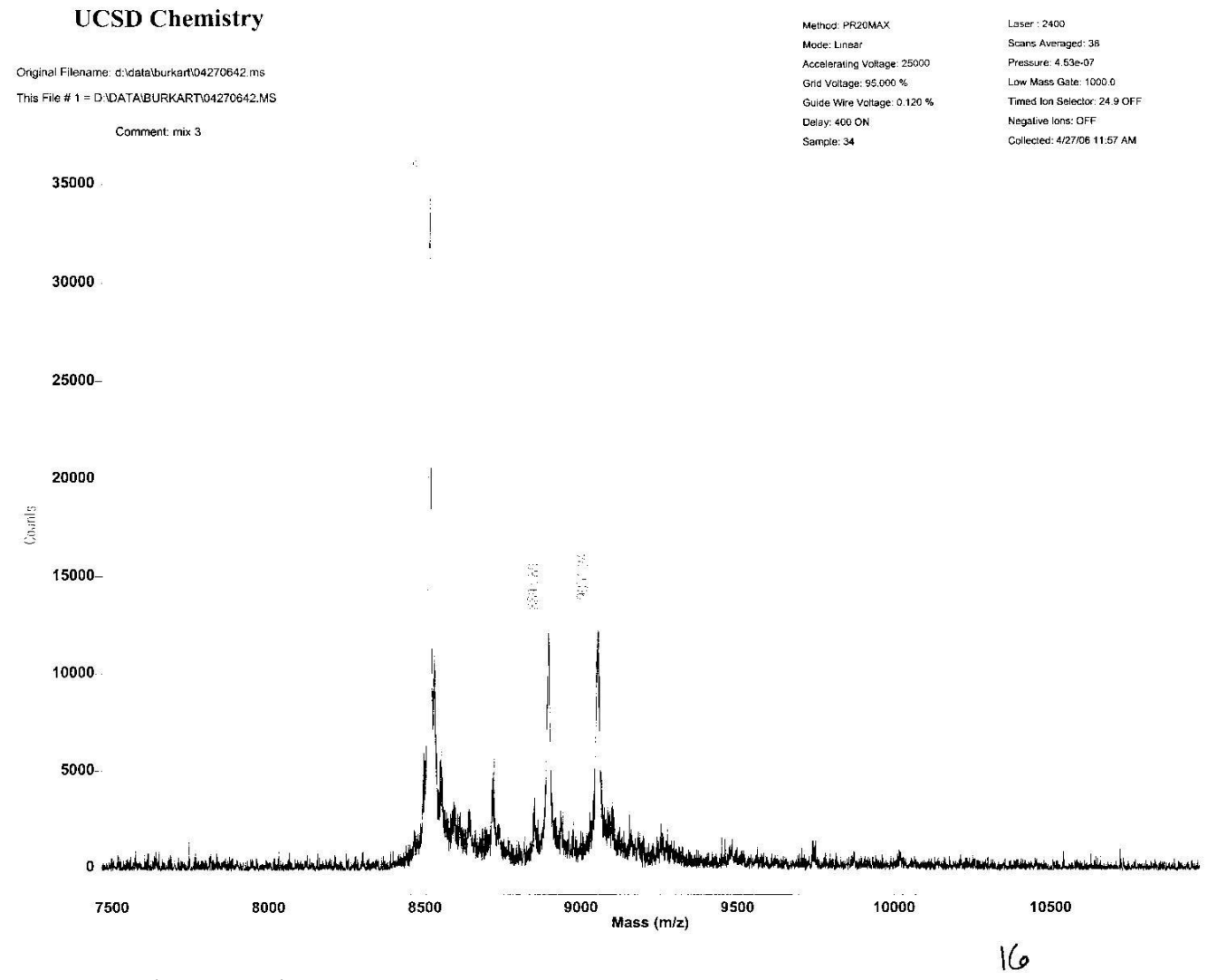

Compound 16 In vivo

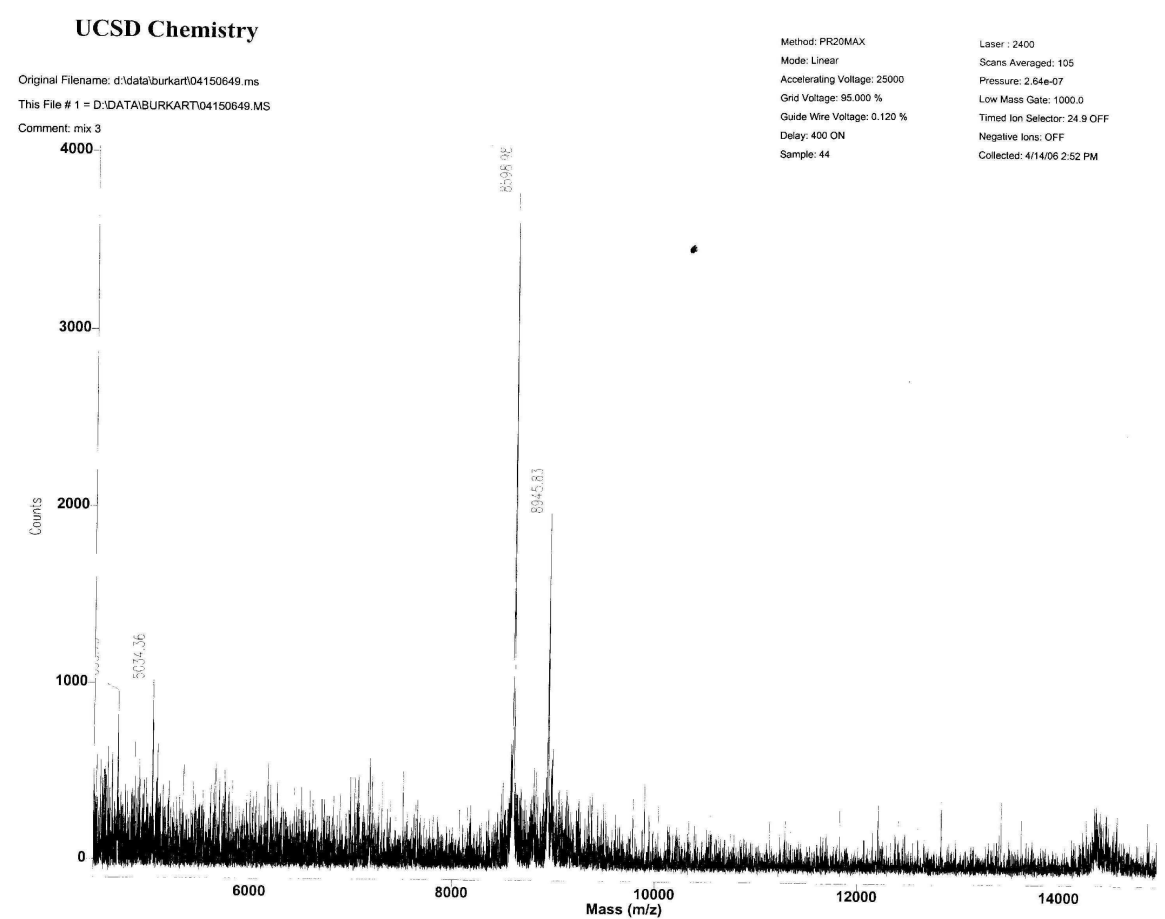

HI 
Compound 17 In vitro

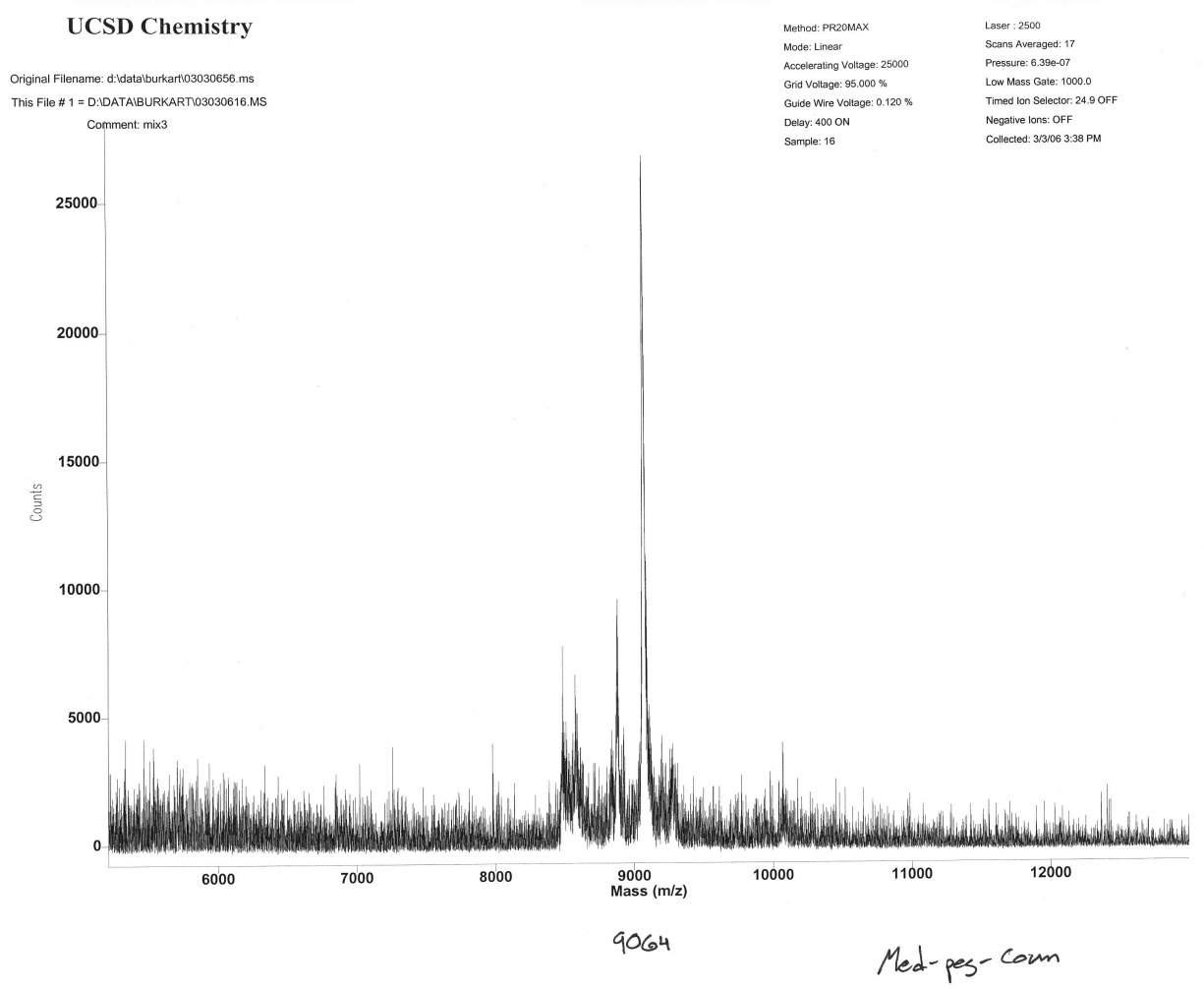

Compound 18 In vitro

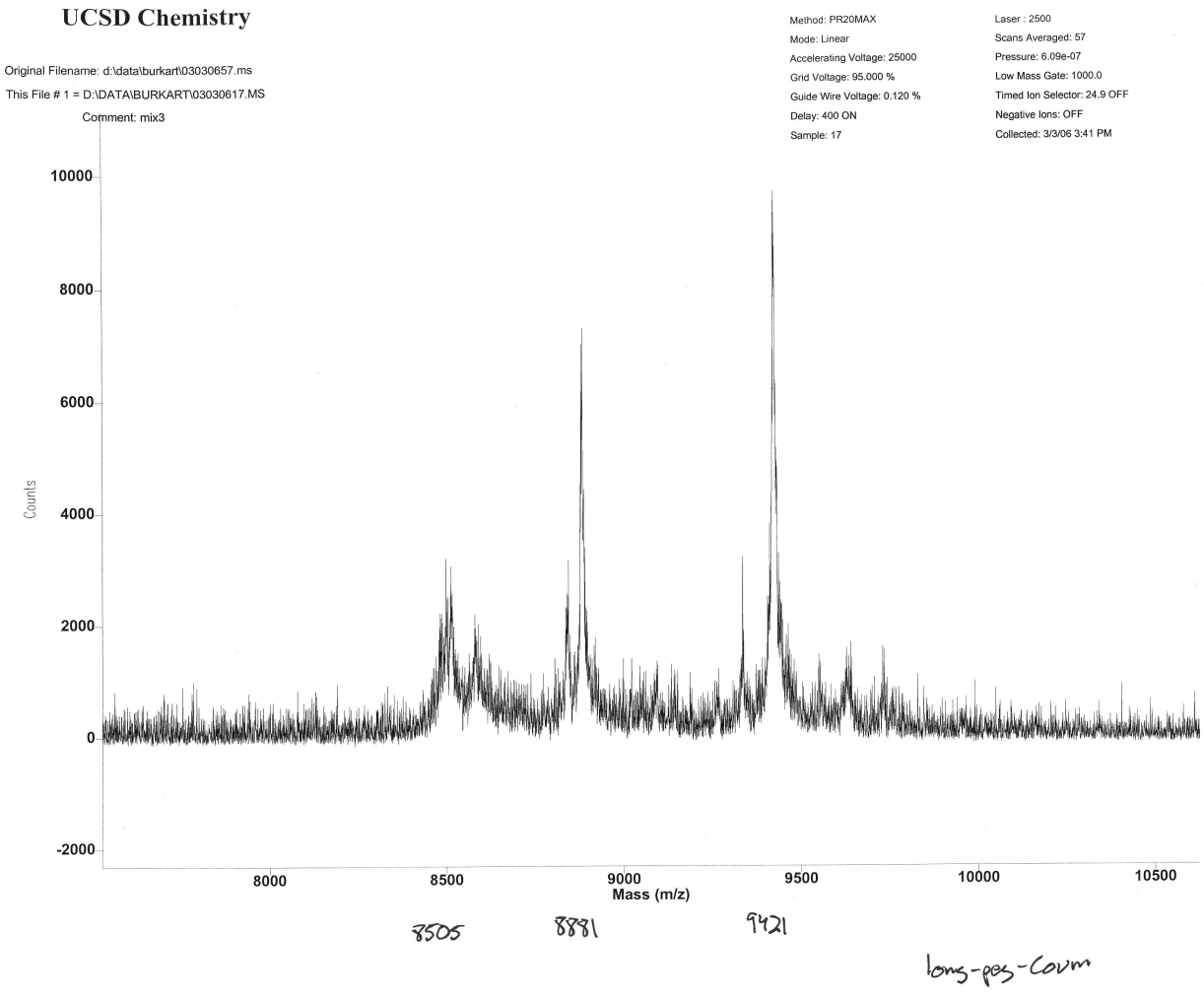


Sulse Sequence: s2pu

Solvent: $\operatorname{CDC} 13$
Temp. $25.0 \mathrm{C}, 298.1 \mathrm{~K}$
UNITY-500 "un500"

Pulse 51.5 degrees

Acq i time 1.892 sec

80 repetitions
OBSERVE H1, $500.3087950 \mathrm{MHZ}$

Line broadening $0.3 \mathrm{~Hz}$

Total time $31 \mathrm{~min}, 42 \mathrm{sec}$

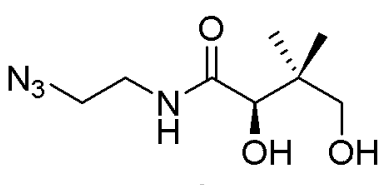

1

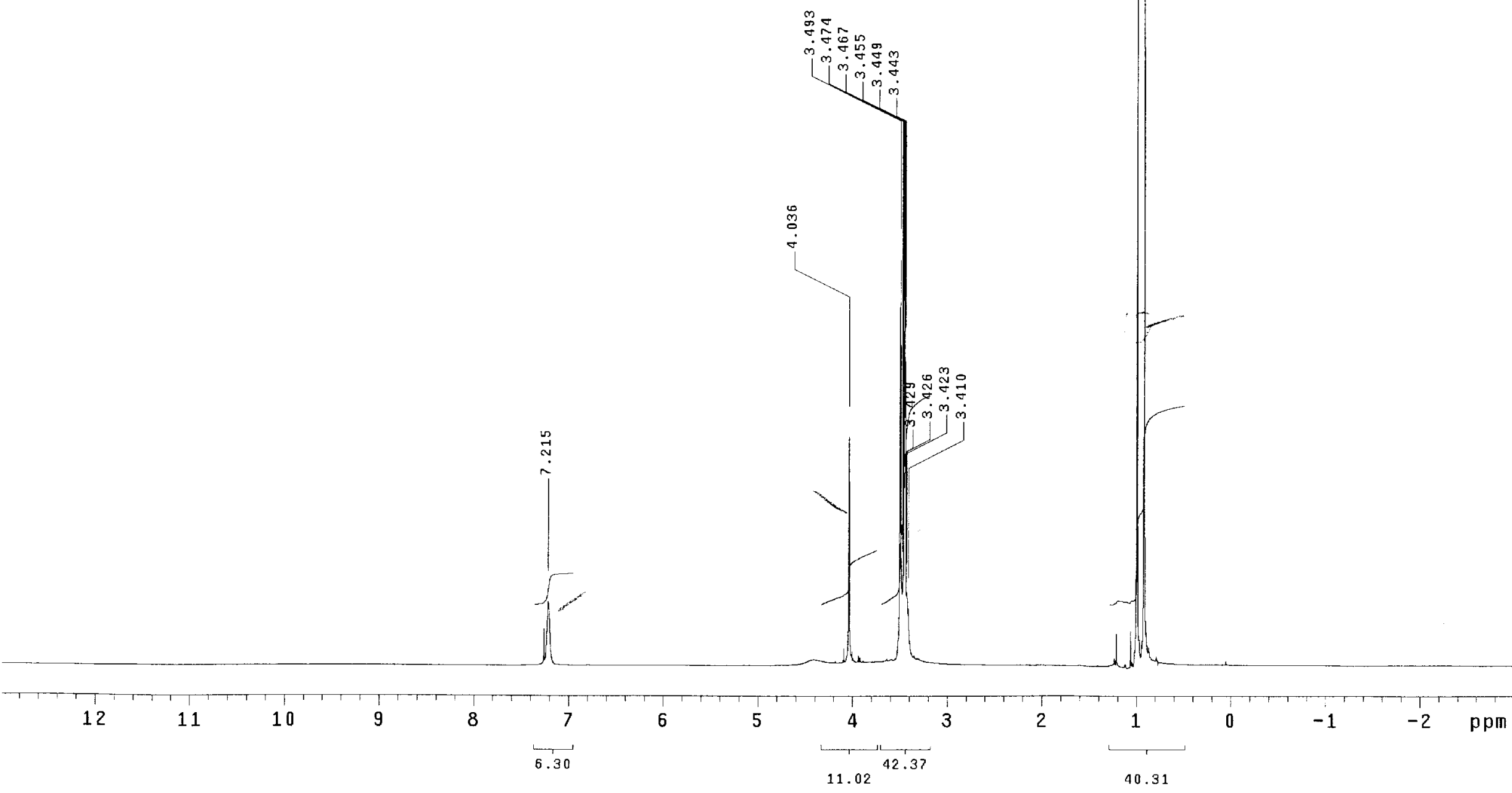


Pülse Sequence: s2pur

Solvent: $\operatorname{CDC} 13$

Ambient temperature

Pulse 60.5 degrees

Acq. $\mathrm{time} 1.815 \mathrm{sec}$

width $18761.7 \mathrm{~Hz}$

944 repetitions

$5.4540290 \mathrm{MHz}$

DECOUPLE H1, 300.0770584 MHz

Power 42 dB

WALTZ 16 sidulated

DATA PROCESSING

DATA proadening

LT ine broaden

$1.0 \mathrm{~Hz}$

Total time $5 \mathrm{hr}, 52 \mathrm{~min}, 1 \mathrm{sec}$

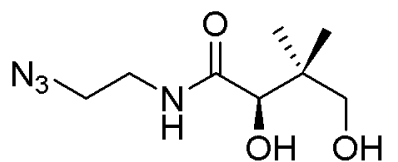

1

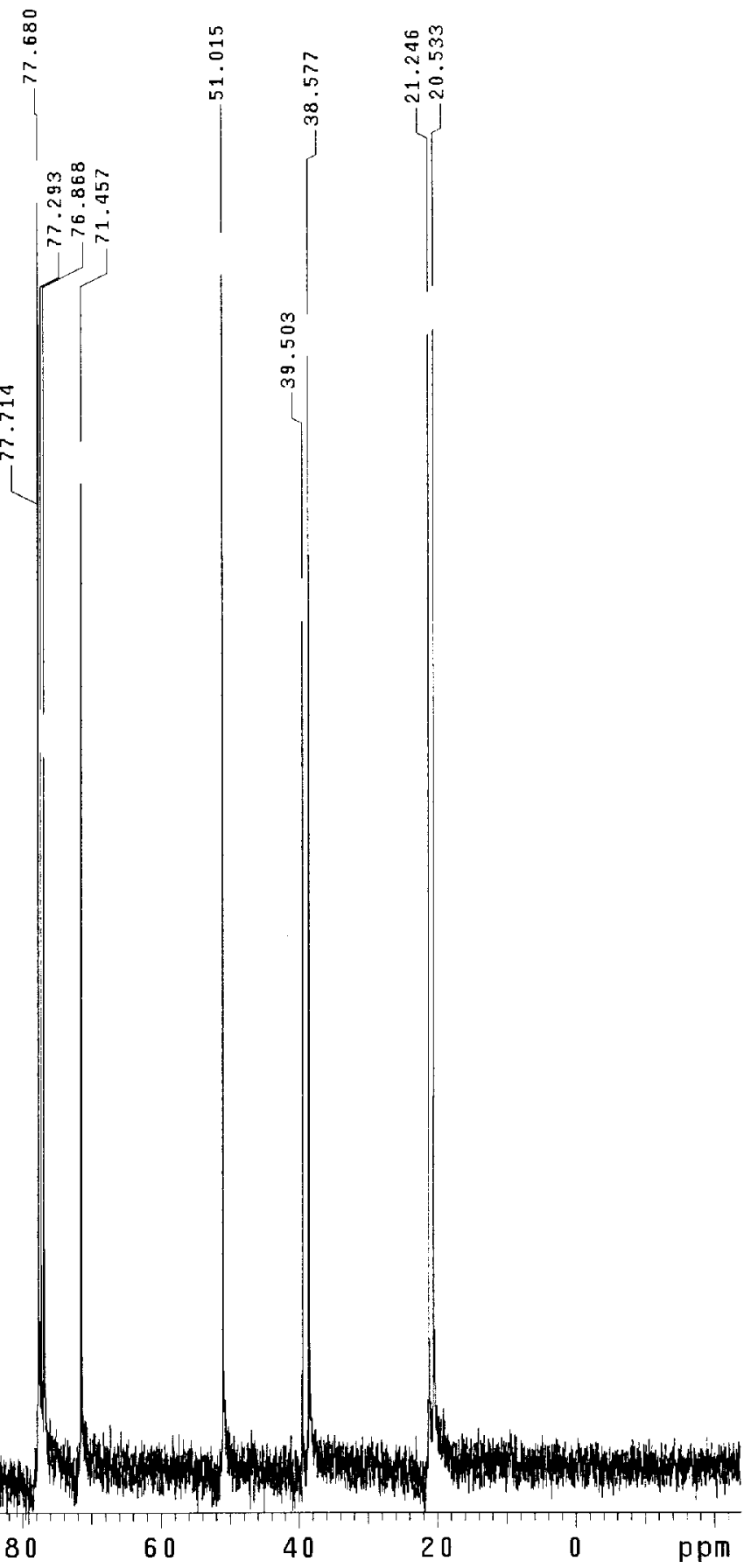


Pulse Sequence: s2pul

Solvent: Acetone

Ambient temperature

Relax. delay $1.000 \mathrm{sec}$

Pulse 12 . degrees

Acq.

40 repetitions

OBSERVE H1, 300.0770676 MHZ

DATA PROCESSING

FT size 32768

Total time $53 \mathrm{~min}, 11 \mathrm{sec}$
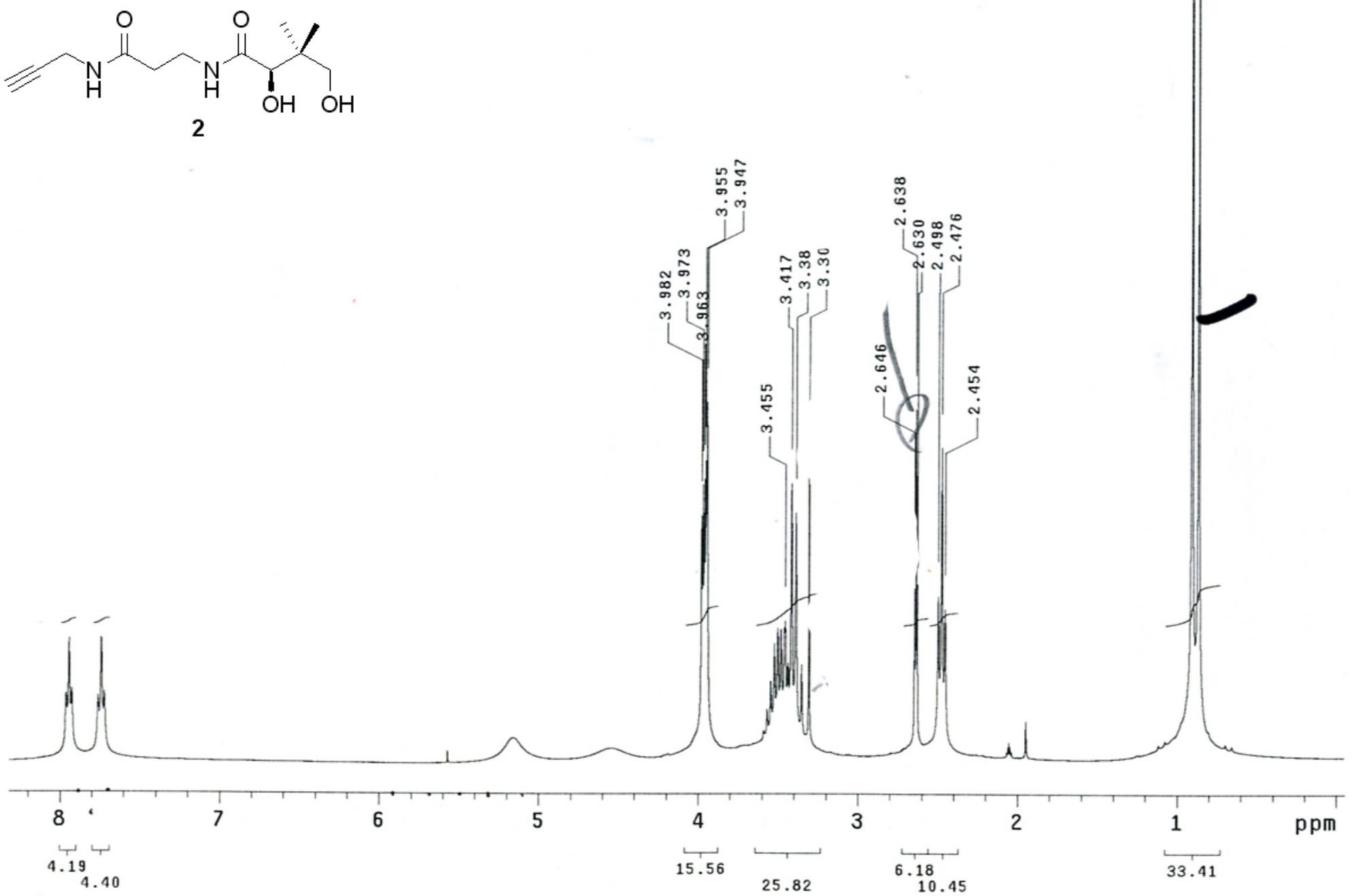
Solvent: Acetone

Ambient temperature
Mercury-300 "hg300"

Pulse 60.5 degrees

Acq. time 1.815 sec

120 repetitions

OBSERVE C13, 75.4544206 MHz

DECRY

cont inuous ly

WALTZ-16 modulated

DATA PROCESSING

Line broadenting

$39 \mathrm{~min}, 38 \mathrm{~s}$
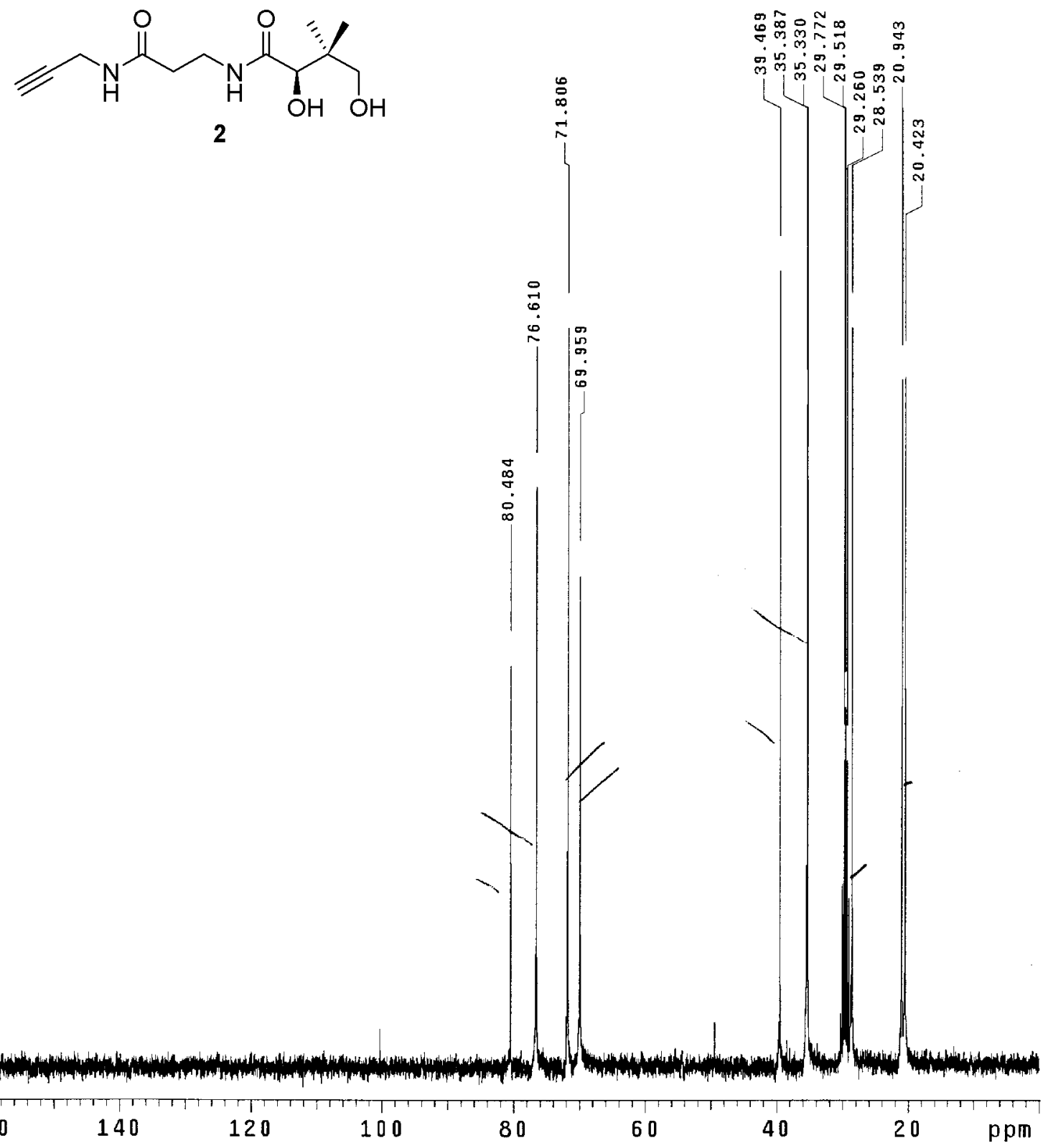


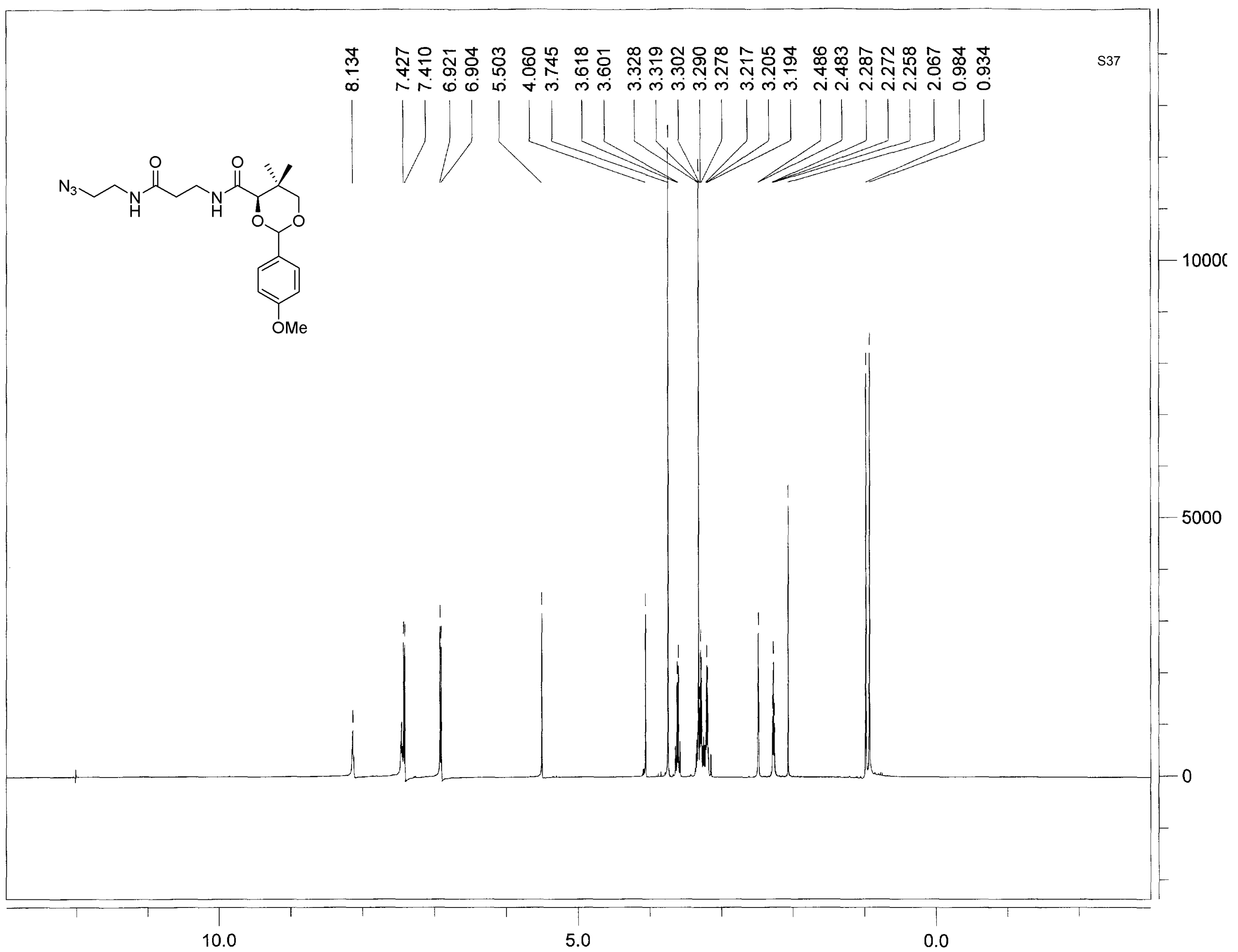


j 1m3-61-monoazi dopantotheni cacid-PMPprotected-CNMRforpub-03-25-06 Pulse sequence: s2pul Solvent: $\operatorname{CDC} 13$ Ambiert temperature Mercury-300 "hg300"

Pulse 60.5 degrees Acq. time $1.815 \mathrm{sec}$ Width $18761.7 \mathrm{~Hz}$ 56 repetitions $4540290 \mathrm{MHz}$ DECOUPLE H1, 300.0770584 MHz Power 42 dB

cont inuousiy on

WALTZ-16 modulated

DATA PROCESSING

Line broadening $1.0 \mathrm{~Hz}$

FT size 131072

Total time $39 \mathrm{~min}, 38 \mathrm{sec}$

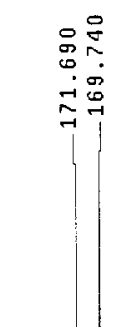

에

$\mid$

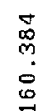

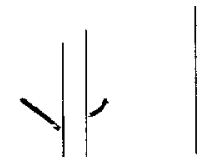

Whind

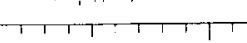

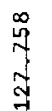

ڤ<smiles>COc1ccc(C2OCC(C)(C)[C@H](C(=O)NCCC(=O)NCCN)O2)cc1</smiles>

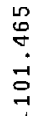

ल.

$\stackrel{m}{\sim}$
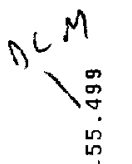

1

ron

节

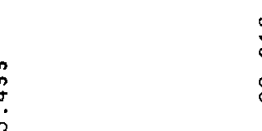

$\stackrel{\infty}{\sim}$

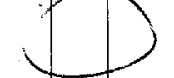


Solvent: $\operatorname{coc} 13$

Ambient temperature
Mercury-400BB "hg402"

Relax. delay $1.000 \mathrm{sec}$ Pulse 45.3 degrees

width $6006.0 \mathrm{~Hz}$

72 repetitions
OBSERVE H1, 400.1233350 MHz

OBSERVE H1,

FT size 32768

Total time $53 \mathrm{~min}, 49 \mathrm{sec}$
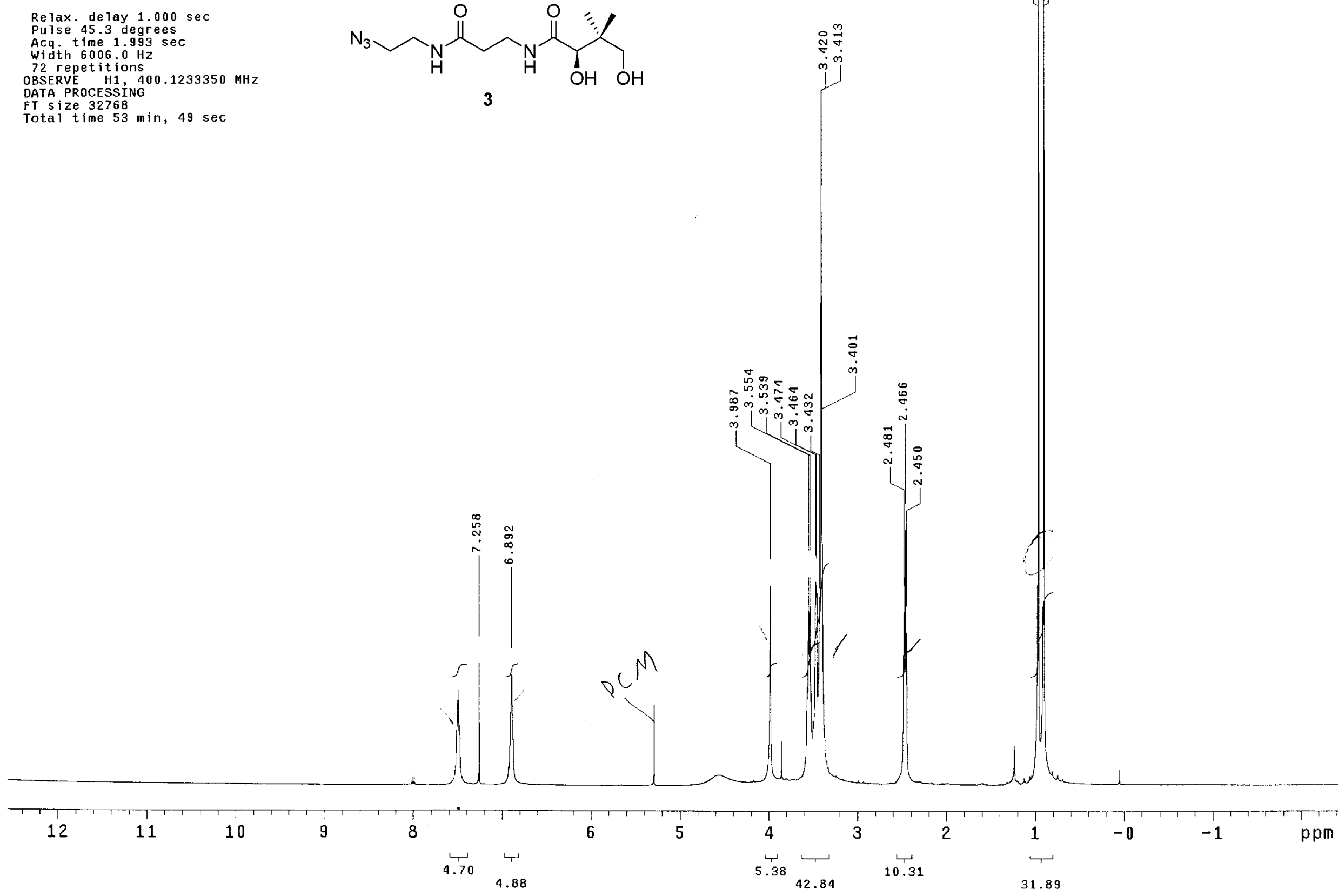

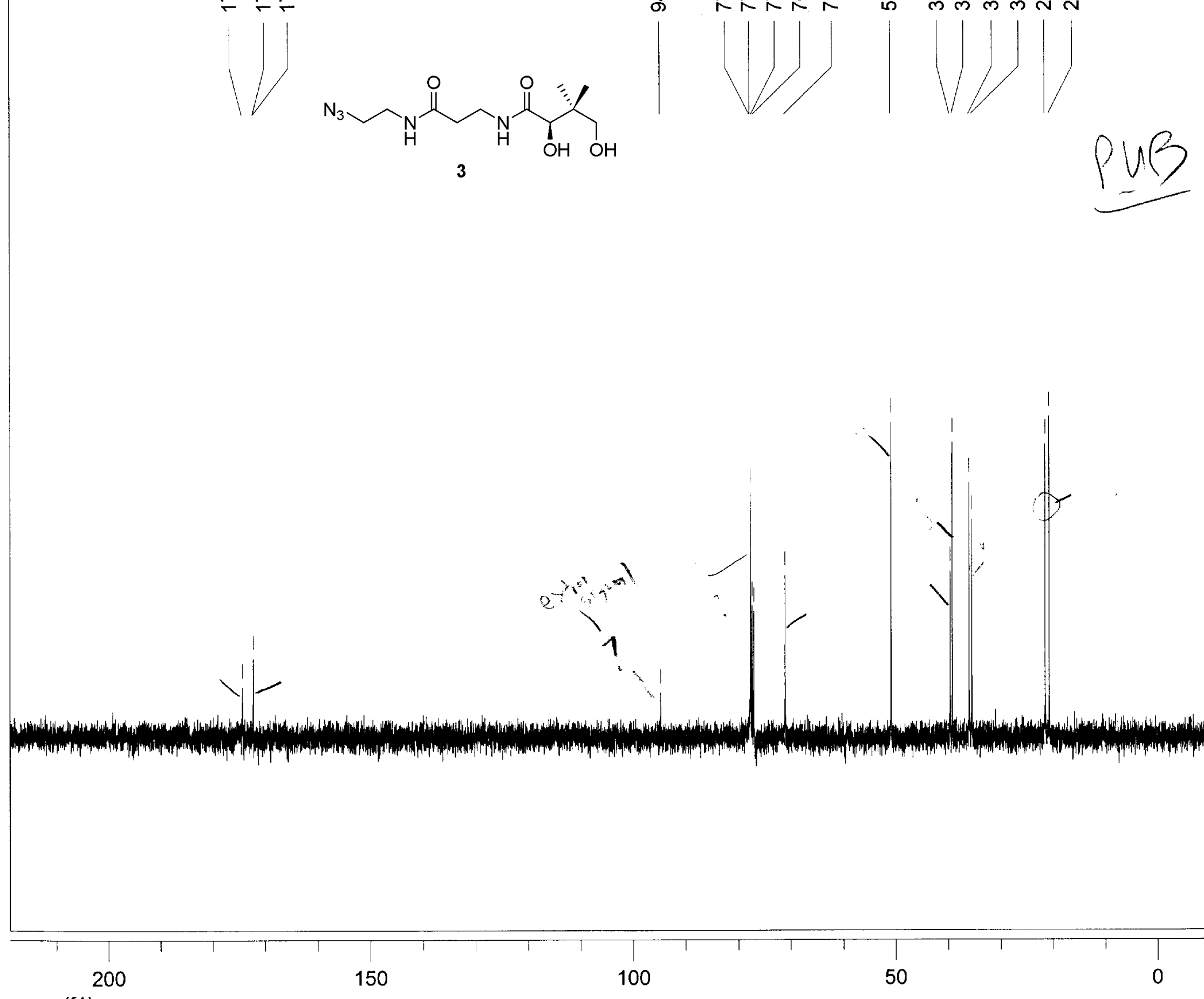

ppm (f1) 
Solvent: $\mathrm{CDCl}^{3}, 298.1 \mathrm{~K}$ UNITY -5

Pulse 32.5 degrees

width $8000.0 \mathrm{~Hz}$

48 repetitions

DATA PROAL, $500.3087950 \mathrm{MHz}$

Line broadening $0.3 \mathrm{~Hz}$

FT size 32768

Total time $31 \mathrm{~min}, 42 \mathrm{sec}$
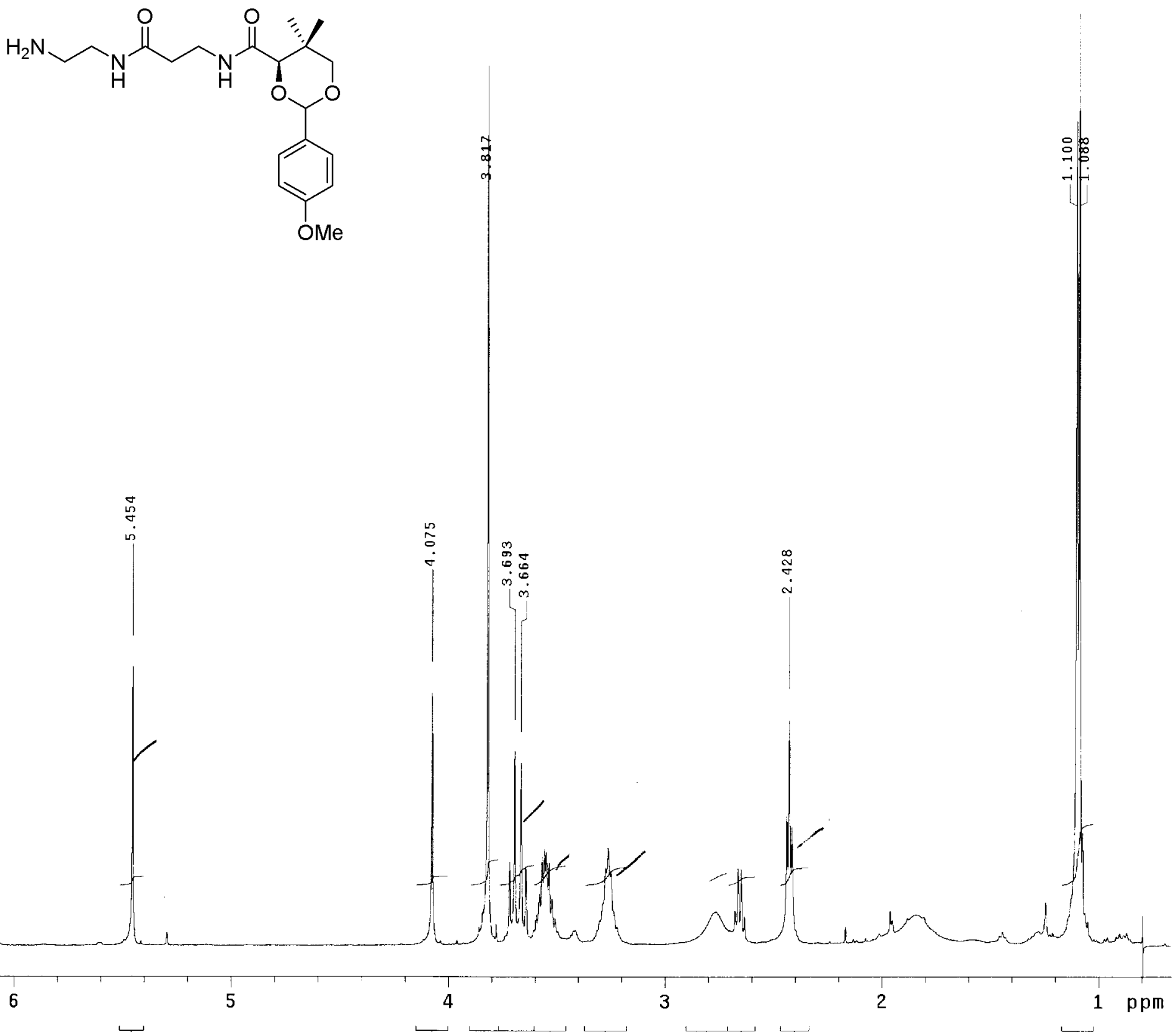

$\begin{array}{lll}6.18 & 5.33 & \\ 6.45 & 2.98\end{array}$

4

$3.65{ }^{10.32} 7.98^{7.78} 7.16$
3

3.53
$5.02 \quad 7.08$ $\stackrel{\infty}{\stackrel{\infty}{*}}$

25.02 
Mercury-300

Pulse 60.5 degrees

Acr. time 1.815 sec

Width $18761.7 \mathrm{~Hz}$

OBSERVE C13, $75.4540290 \mathrm{MHZ}$

DECOUPLE H1, 300.0770584 MHZ

Power 42 dB

Cont

DATA PROCESSING

Line broadening $1.0 \mathrm{~Hz}$

FT 5 ize 131072
Total time $6 \mathrm{hr}, 36 \mathrm{~min}, 23 \mathrm{sec}$
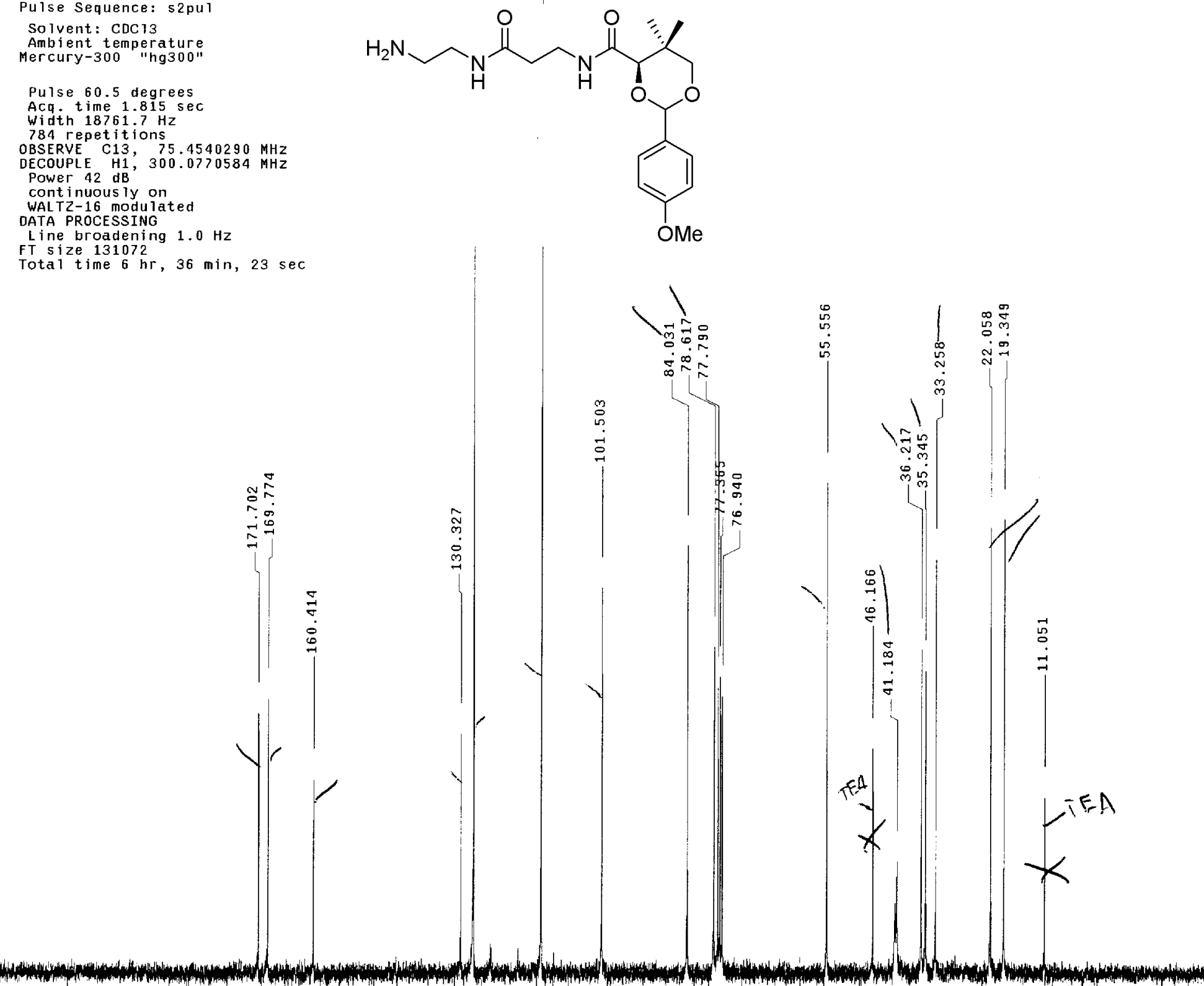


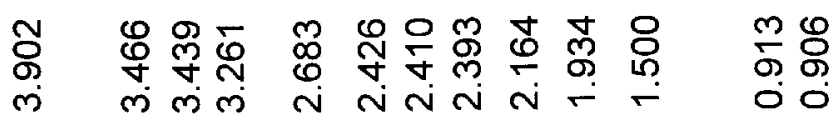

$\mathrm{HOOH}$
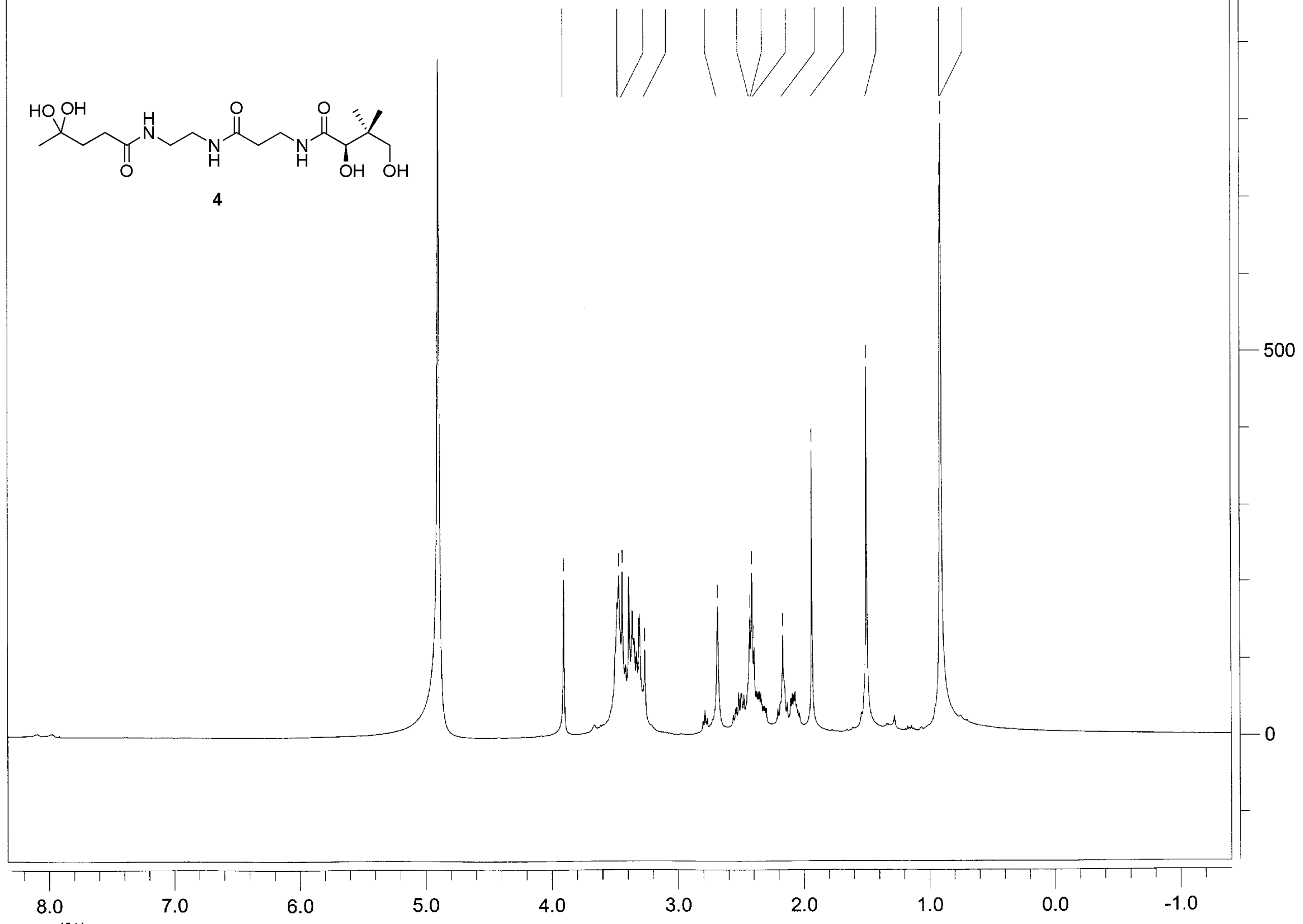

ppm (f1) 
Pulse Sequence: s2pul

Solvent: D20

Temp. $29.0 \mathrm{C} / 302.1 \mathrm{~K}$
Mercury-400B

Pulse 53.4 degrees

Acq. time $1.199 \mathrm{sec}$

width $250000 \mathrm{~Hz}$

9880 repetitions

OBSERVE C13, $100.6113272 \mathrm{MHZ}$

DOWPL H1, 400.1263885 MH

Power $38 \mathrm{~dB}$

WALTZ-16 modulated

DATA PROCESSING

Line broadening $1.0 \mathrm{~Hz}$

FT size 65536

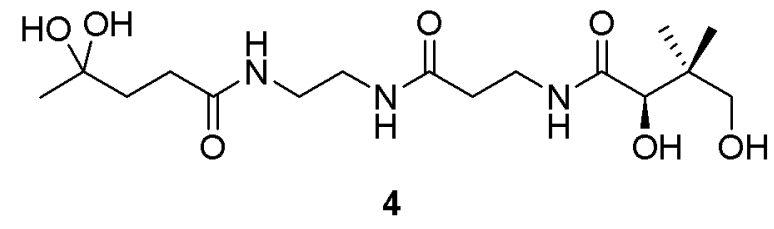

$472 \mathrm{hr}, 58 \mathrm{~min}, 28 \mathrm{sec}$

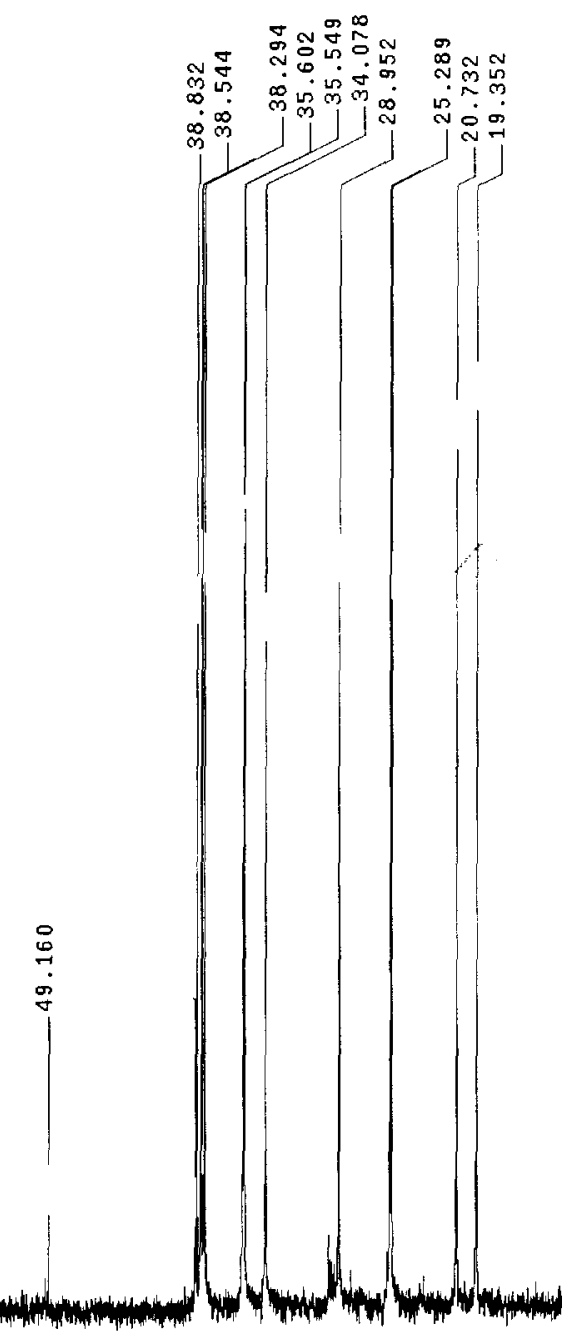

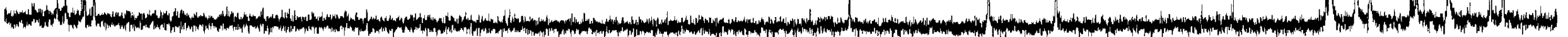

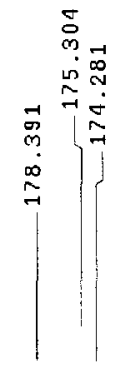


j 1m3-59-traditiona 11 inker-NHSazide 1 inkage-PMP-02-13-06

Pulse Sequence: s2pul

Solvent: $\operatorname{CoC} 13$

Ambient temperature

Relax. delay $1.000 \mathrm{sec}$

Pulse 45.3 degrees

Width 4506.5 Hz

40 repetitions

OBSERVE H1, $300.0755102 \mathrm{MHZ}$

DATA PROCESSING

Tatal time $0 \mathrm{~min}, 0 \mathrm{sec}$
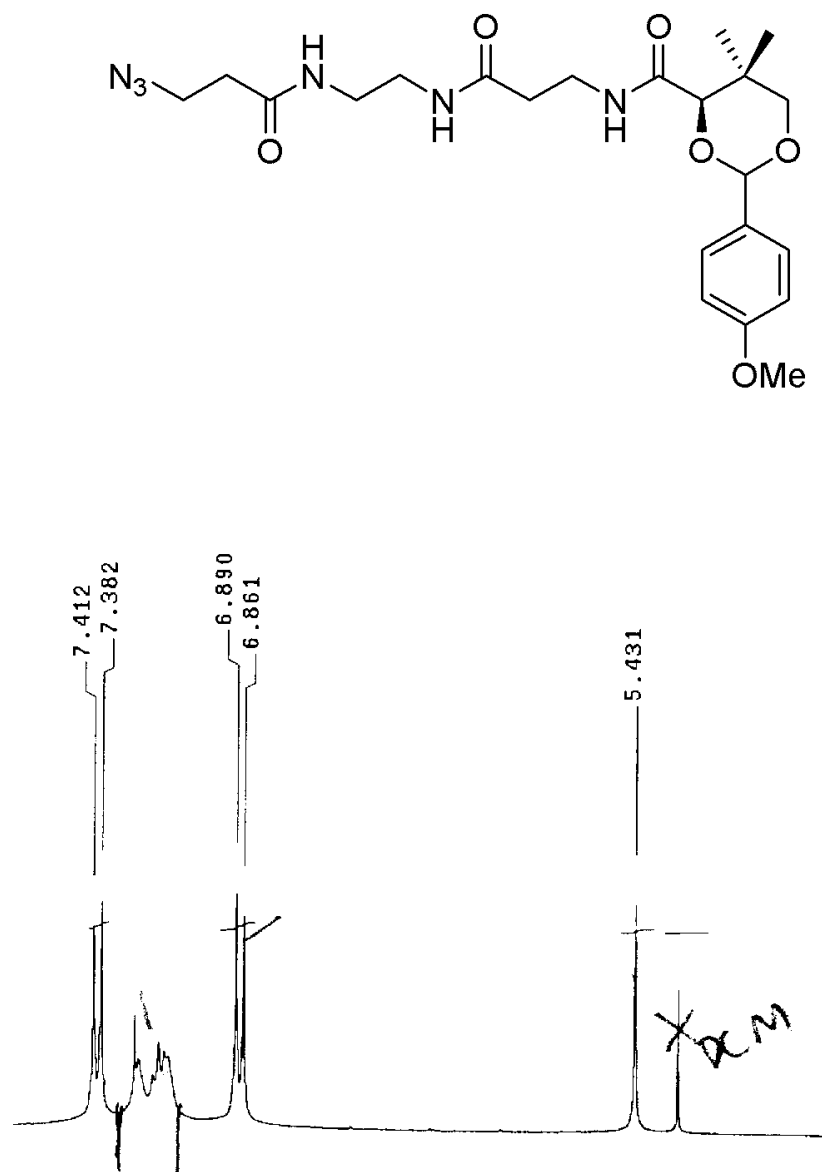

$\vec{y}$

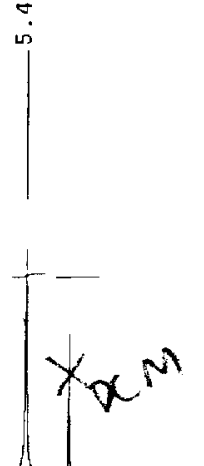

5

4.48 4.60

1.87 .38

$2.13 \begin{aligned} & 7.6611 .70 \\ & 9.4510 .58\end{aligned}$

13.82

ppm

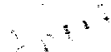


Pulse Sequence: s2pul Solvent: $\operatorname{CDC} 13$

Ambient temperature
Mercury- 300 "hg $300 "$

Pulse 60.5 degrees

Acq. time $1.815 \mathrm{se}$

Width 18761 ions

OBSERVE C13, $75.4540290 \mathrm{MHZ}$

DECOUPLE H1, 300.0770584 MHZ

Power $42 \mathrm{~dB}$

WALTZ-16 modulated

DATA PROCESSING

Line broadening $1.0 \mathrm{~Hz}$

FT size 131072
Total time $39 \mathrm{~min}, 38 \mathrm{sec}$
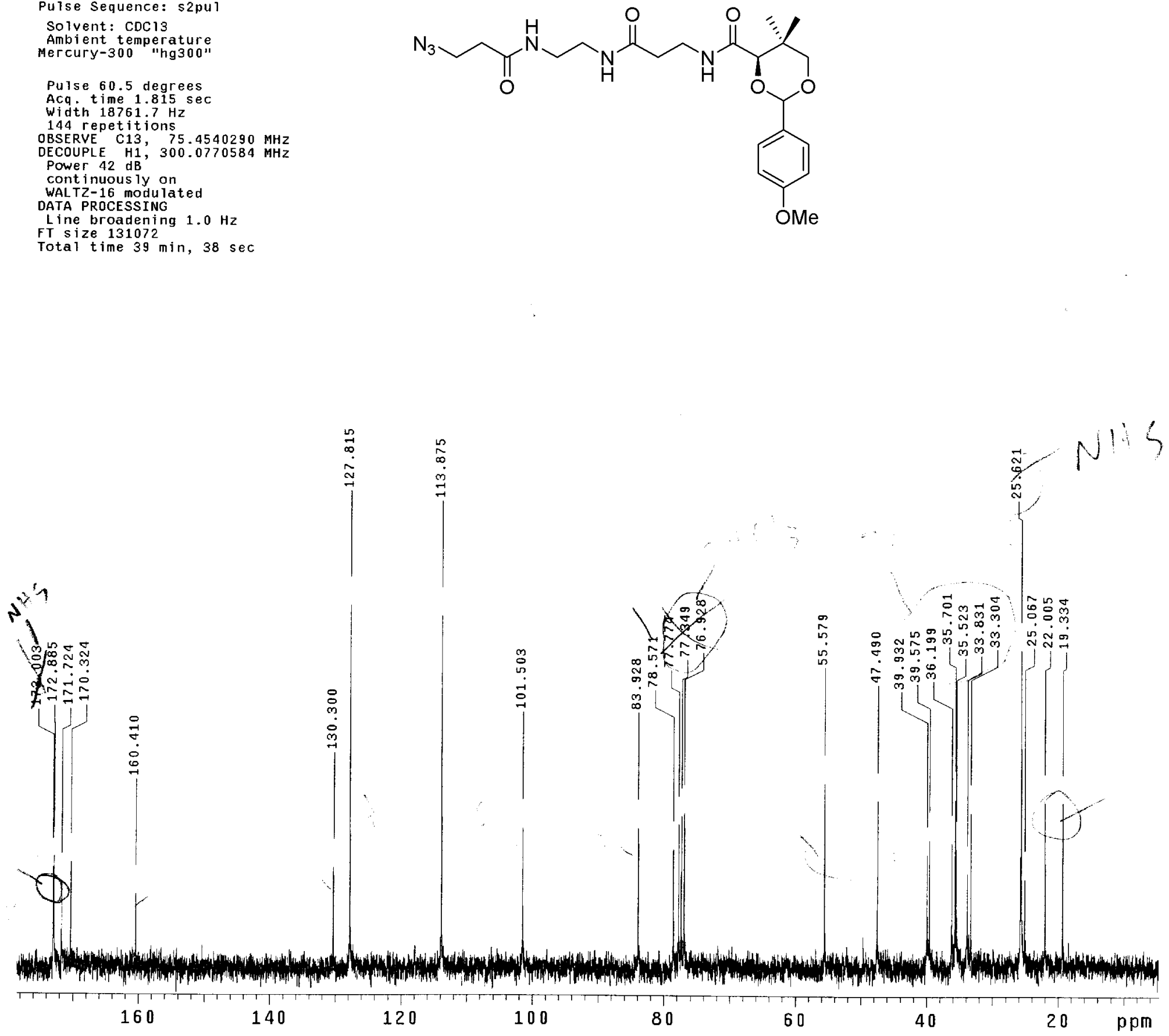
Solvent: Acetone

Temp. $25.0 \mathrm{C} / 298.1 \mathrm{~K}$

Puise 51.5 degrees

Acq. time 1.892 sec

136 repetitions

OBSERVE H1, 500.3113916 MHZ

DATA PROCESSINO

Line broadening $0.3 \mathrm{~Hz}$

Total time $31 \mathrm{~min}, 42 \mathrm{sec}$
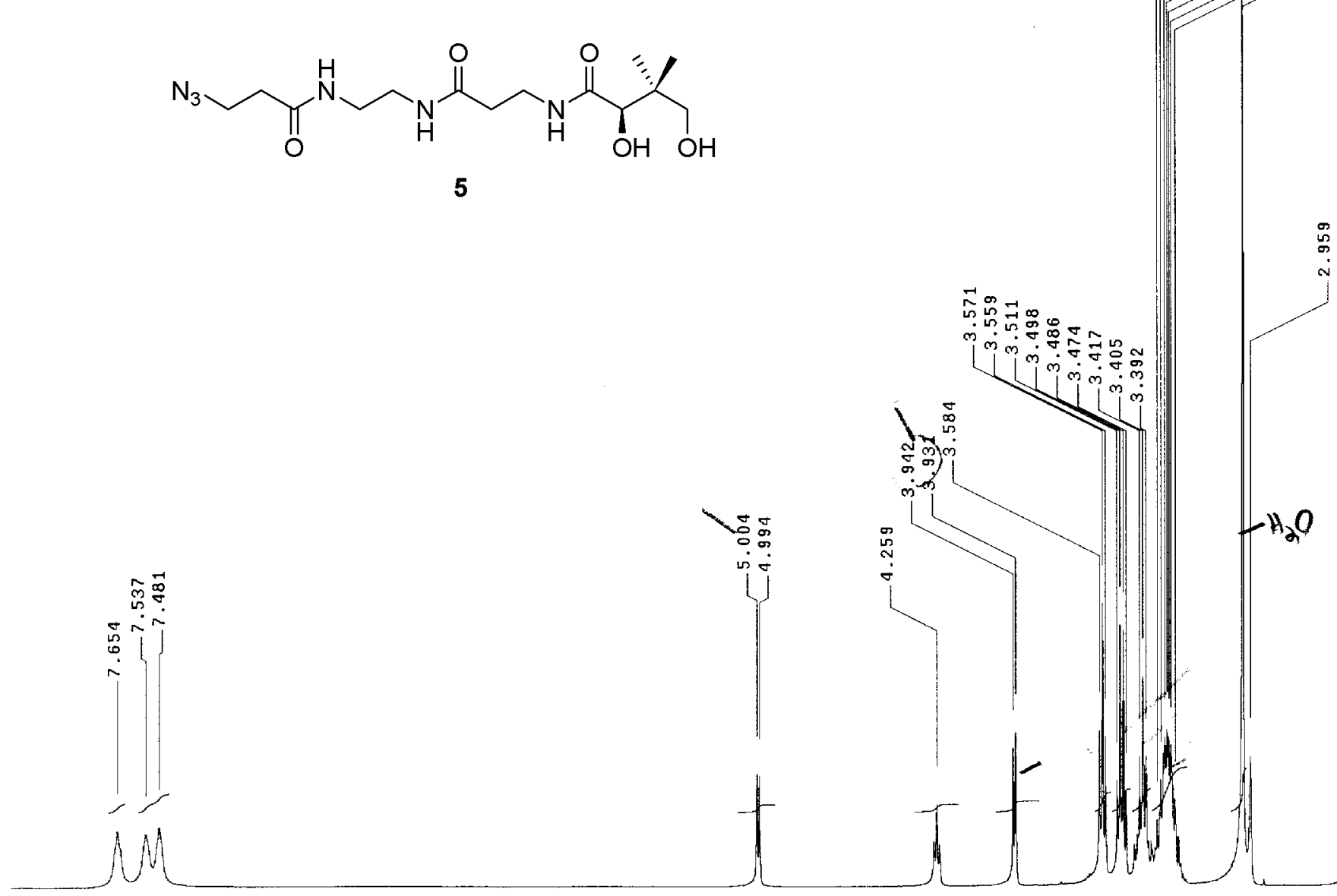

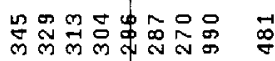

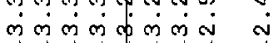


Pudise sequence: s2pur

Solvent: Acetone

Aqbient temperature
Meqcury-300 "hg300"

Pulse 60.5 degrees

Acq. time $1.815 \mathrm{sec}$

Width $18761.7 \mathrm{~Hz}$

896 repetitions

OBSERVE C13, 75.4544206 MHZ

DEGOUPLE H1, 300.0786158 MHZ

Power 42 dB

cont inuous iy on

WALTZ-16 modulated

DATA PROCESSING

Line broadening $1.0 \mathrm{~Hz}$

Totat time $39 \mathrm{~min}, 38 \mathrm{sec}$
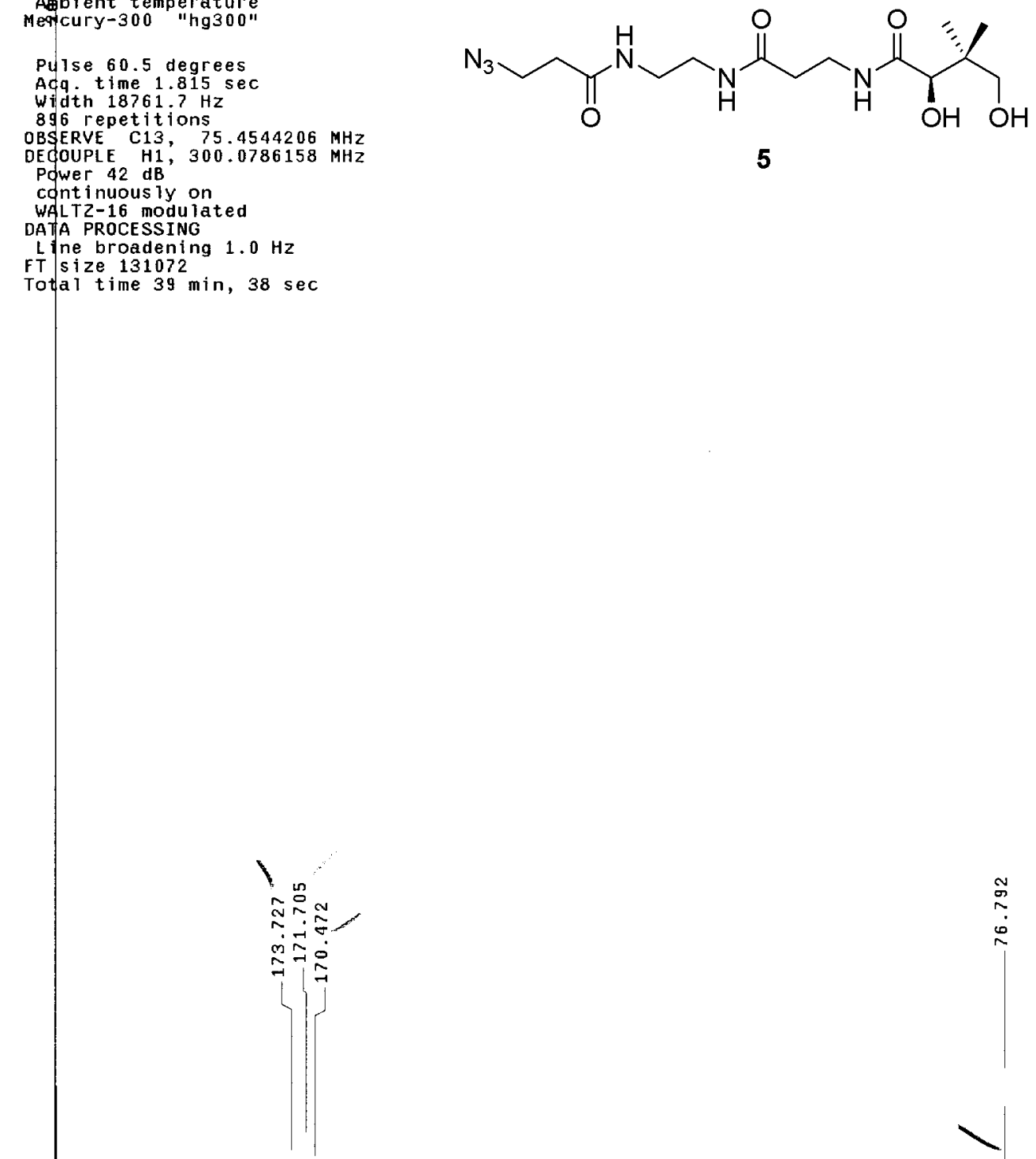

$\stackrel{5}{\stackrel{5}{2}} \stackrel{5}{\pi}$

$\stackrel{2}{\sim}$

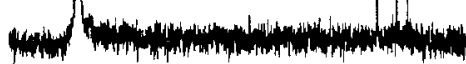

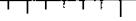

180

160

140

120

100

80

ppm 


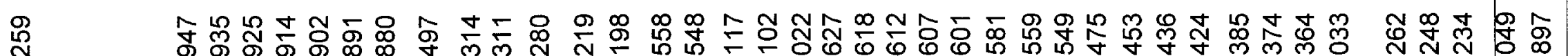
N $\quad$ ம்<smiles>C=CCOC(=O)NCCOCCOCCNC(=O)[C@H](O)C(C)(C)CO</smiles>
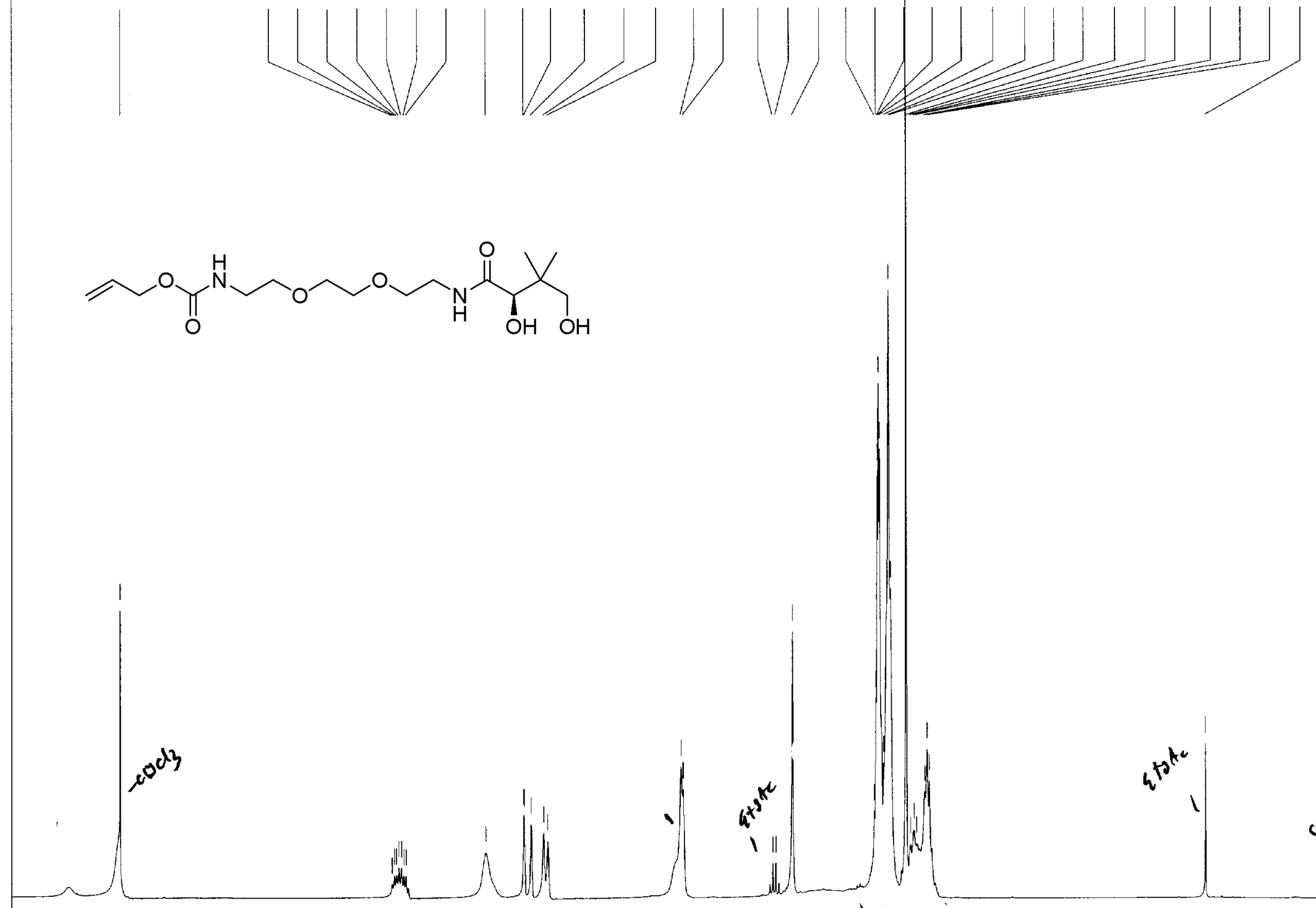

(N)
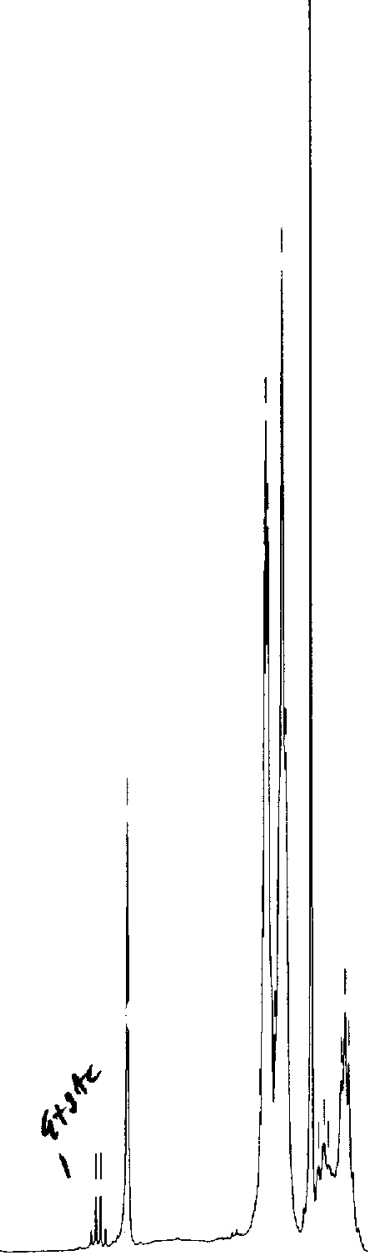
Solvent: $\operatorname{CDC} 13$

Temp. $25.0 \mathrm{C} / 298.1 \mathrm{~K}$
UNITY- 500 "un $500^{\circ 1}$

Pulse 32.5 degrees

Acq. time 1.892 sec

Width $8000.0 \mathrm{HZ}$
56 repetitions

OBSERVE H1, 500.3087950 MHZ

DATA PROCESSING

FT siz 32768

rotal time $31 \mathrm{~min}, 42 \mathrm{sec}$
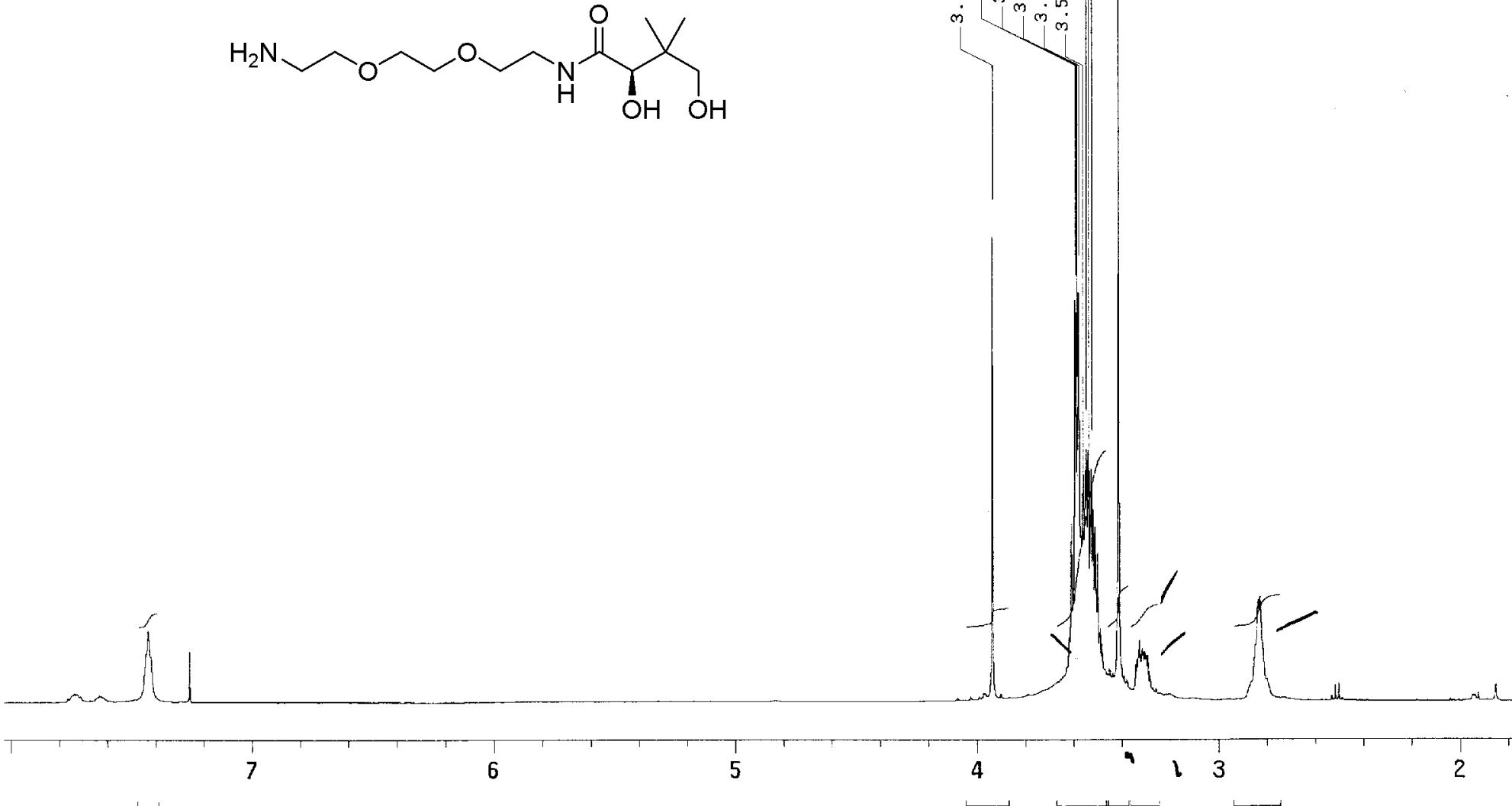


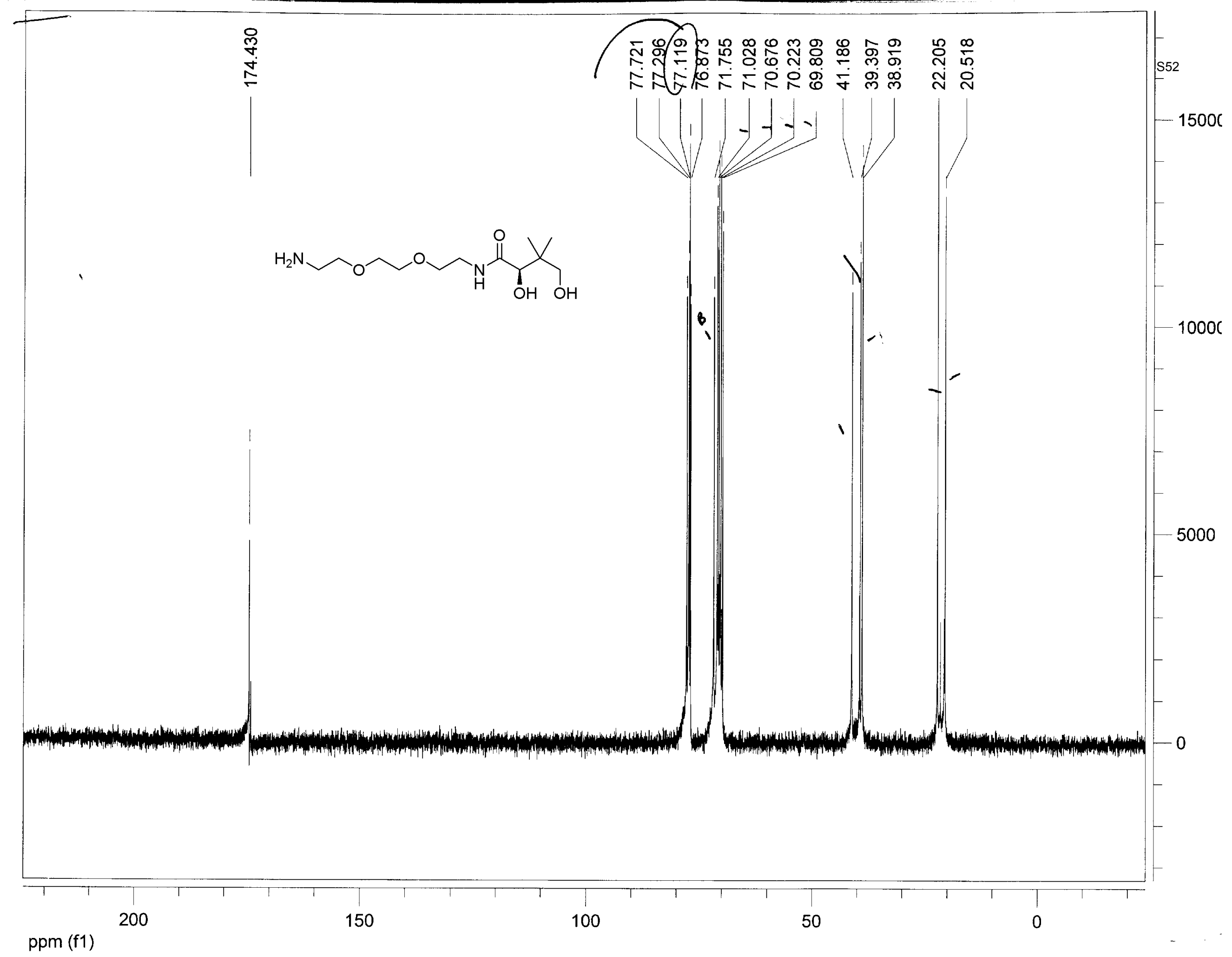


Solvent: $\mathrm{CDCl}^{2} 3298 \mathrm{~K}$
Temp. $25.0 \mathrm{C} / 298.1 \mathrm{~K}$

\section{Pulse 51.5 degrees}

Acq. time $1.892 \mathrm{sec}$

Width $8000.0 \mathrm{~Hz}$

OBSERVE H1, 500.3087950 MHZ

DATA PROCESSINC

Line broadening $0.3 \mathrm{~Hz}$

Total time $31 \mathrm{~min}, 42 \mathrm{sec}$

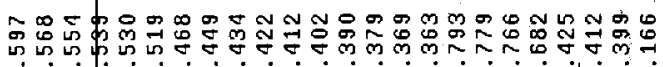

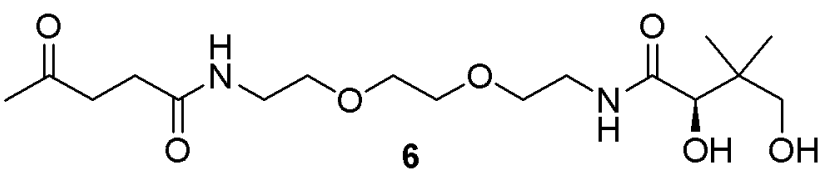

6
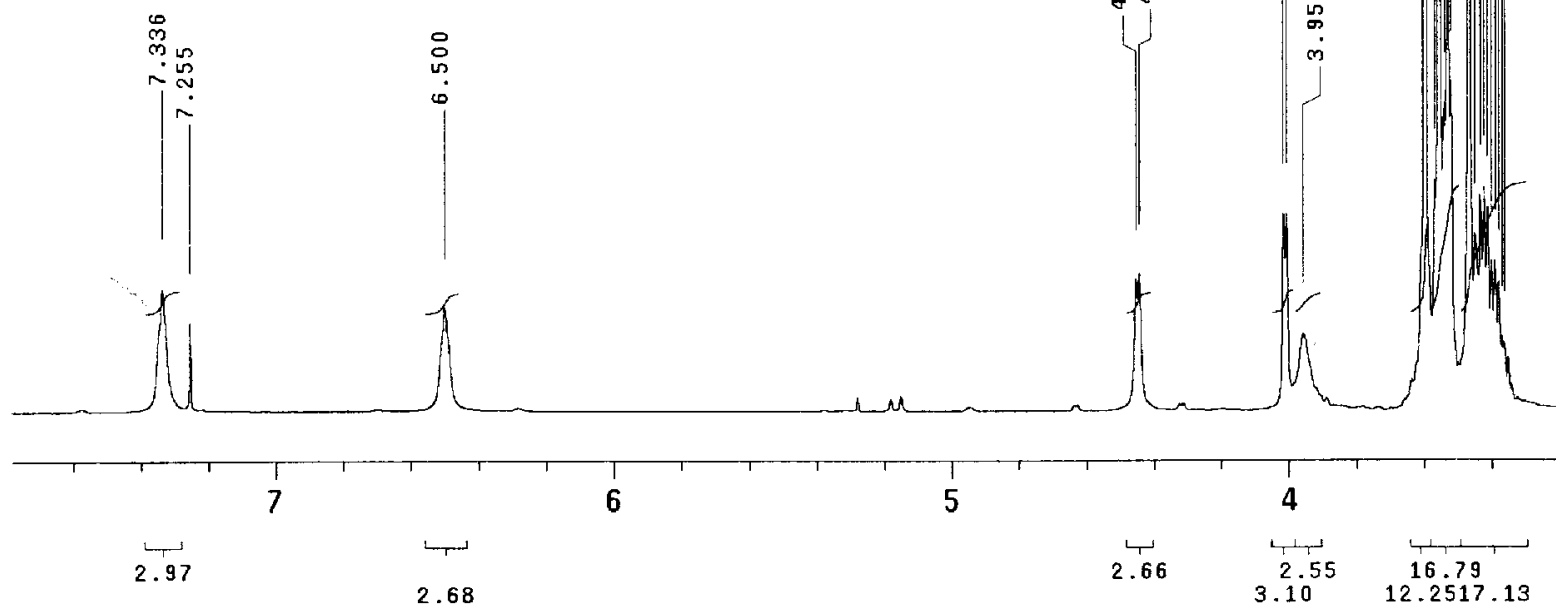

$\begin{array}{lll}5.71 \\ 1.04 & 5.68 & 4.51\end{array}$ 
Mercury-400BB "hg402"

Pulse 53.4 degrees
Acq. time $1.19 g$ sec

Width $25000.0 \mathrm{~Hz}$

OBSERVE C13, 100.6110929 MHZ

DECOUPLE H1, 400.1253602 MHZ

Power $38 \mathrm{~dB}$

WALTZ-16 modulated

WALTZ-16 modu Tated

Line broadening $1.0 \mathrm{~Hz}$

FT size 65536
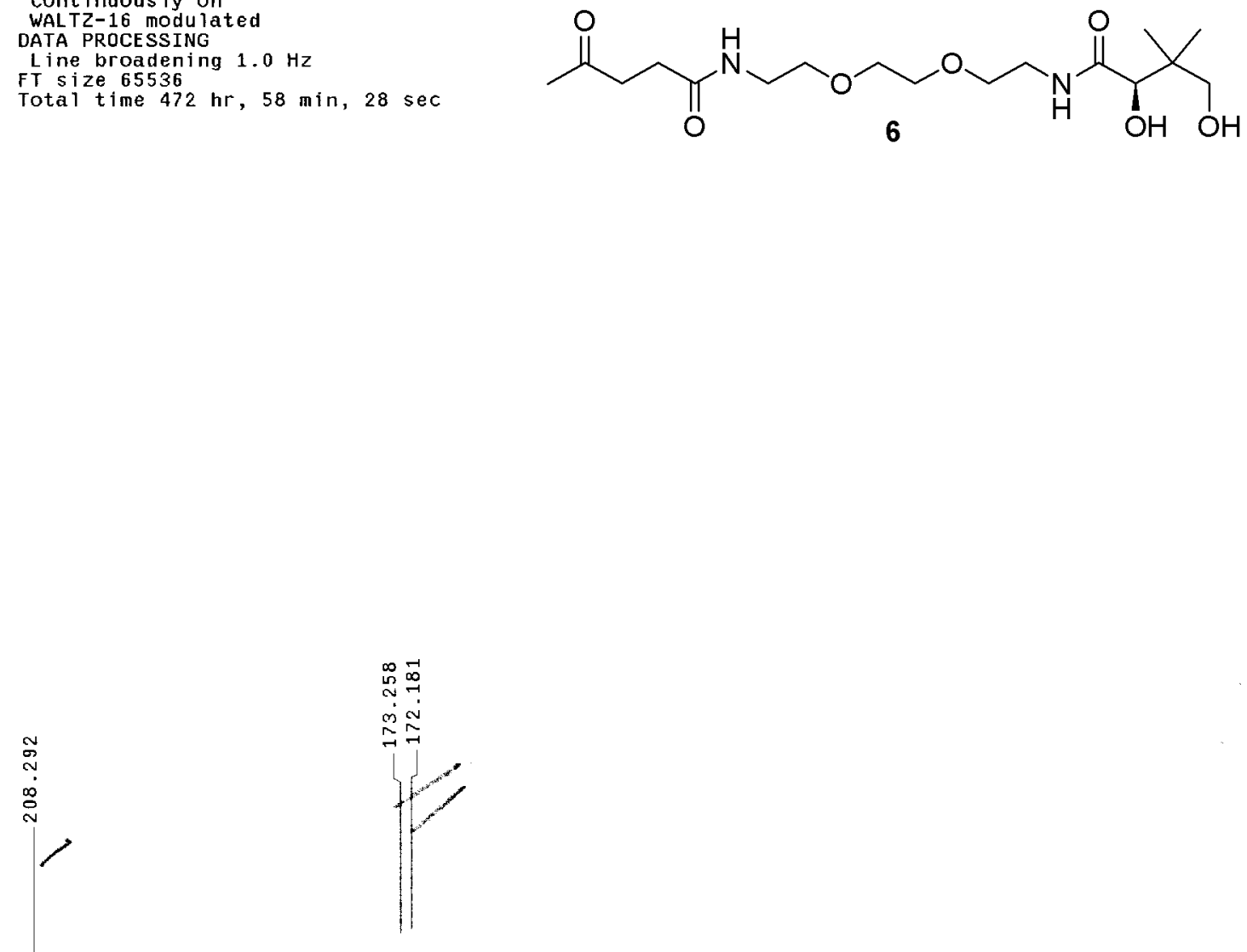

6

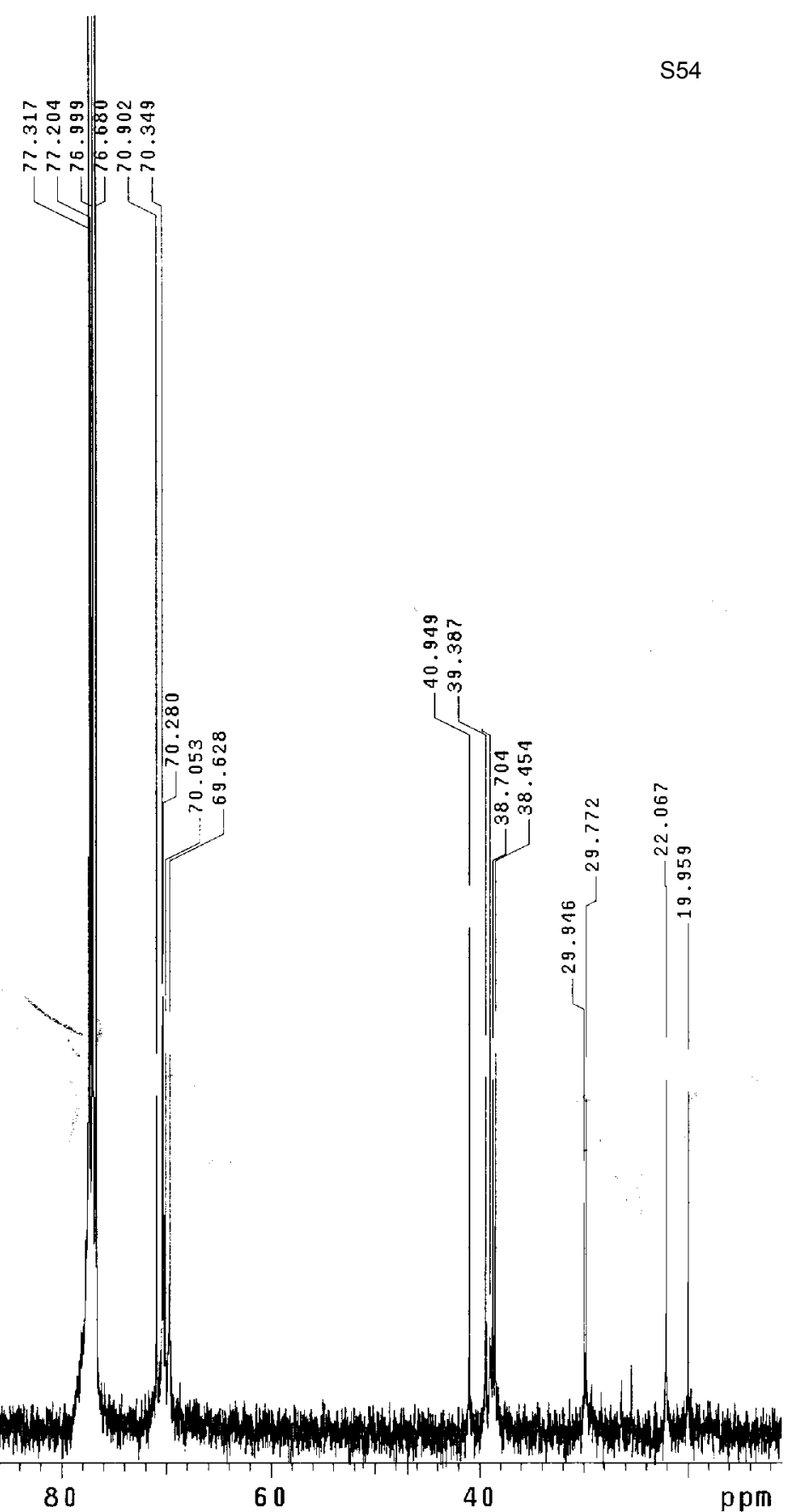


Solvent: $\operatorname{CDC} 13$
Temp. $25.0 \mathrm{C} / 298.1 \mathrm{~K}$
UNITY-500 "un500"

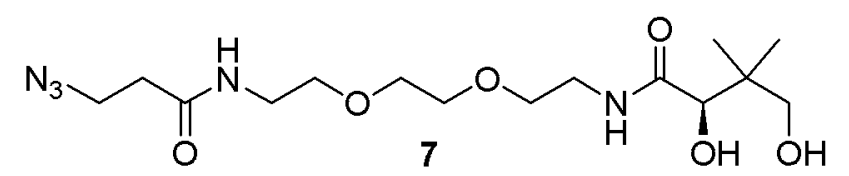

Pulse 51.5 degrees
Acq. time 1.892 sec

Acq. time 1.892
width $8000.0 \mathrm{~Hz}$

72 repetitions $0003950 \mathrm{MHZ}$

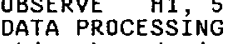

Line broadening $0.3 \mathrm{~Hz}$

Tota? time $31 \mathrm{~min}, 42 \mathrm{sec}$
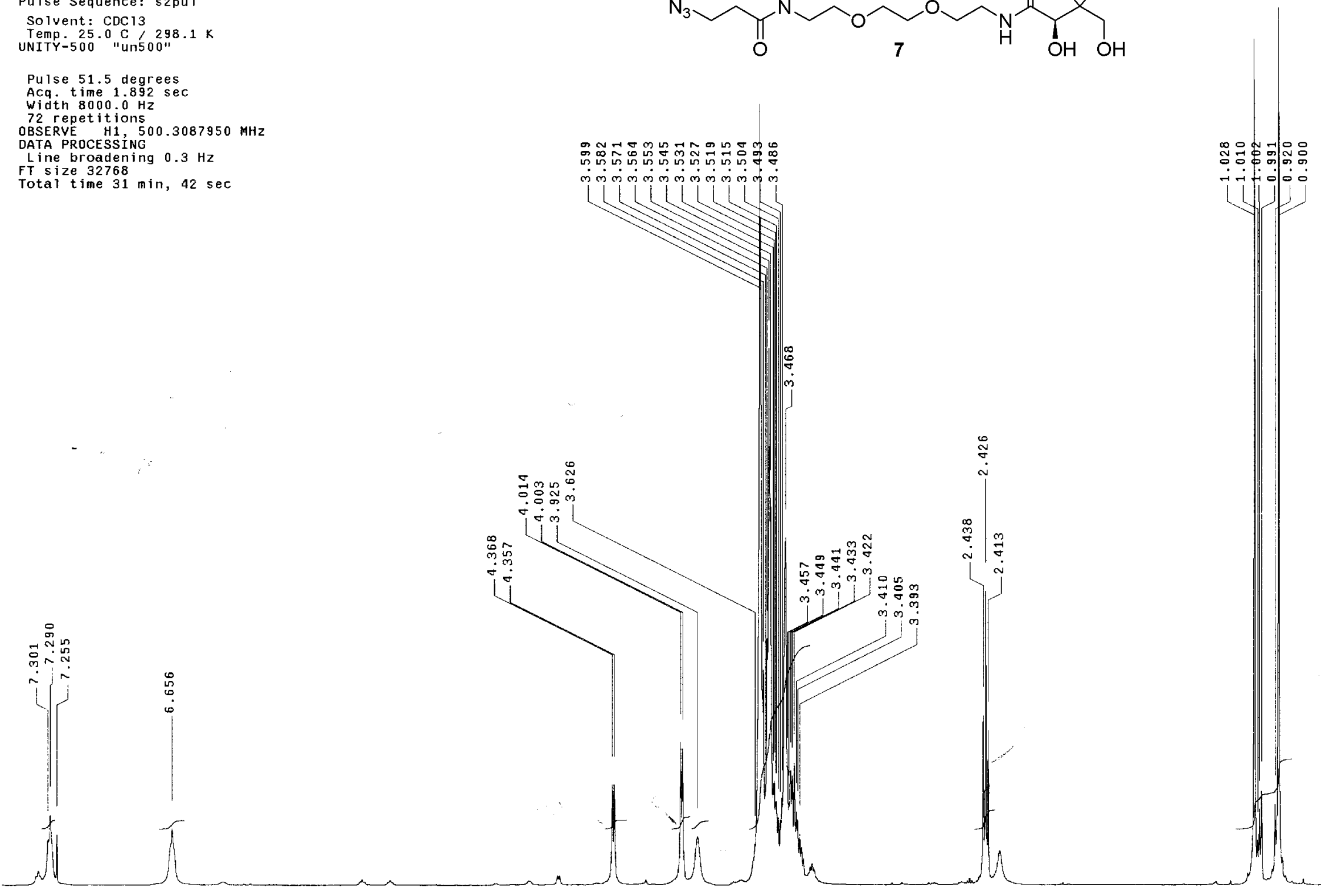
Temp. $25.0 \mathrm{C} / 298.1 \mathrm{~K}$

Pulse 51.5 degrees

Acq. time 1.892 sec

Width $8000.0 \mathrm{~Hz}$

OBSERVE H1, $500.3087950 \mathrm{MHz}$

DATA PROCESSING $0.3 \mathrm{~Hz}$

Line broadening $0.3 \mathrm{~Hz}$

FT size 32768
Total time $31 \mathrm{~min}, 42 \mathrm{sec}$

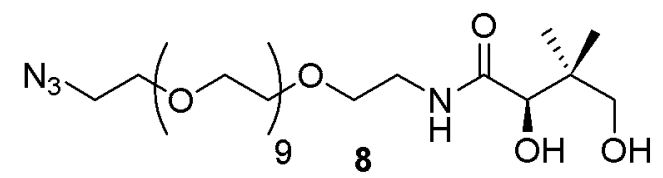

is




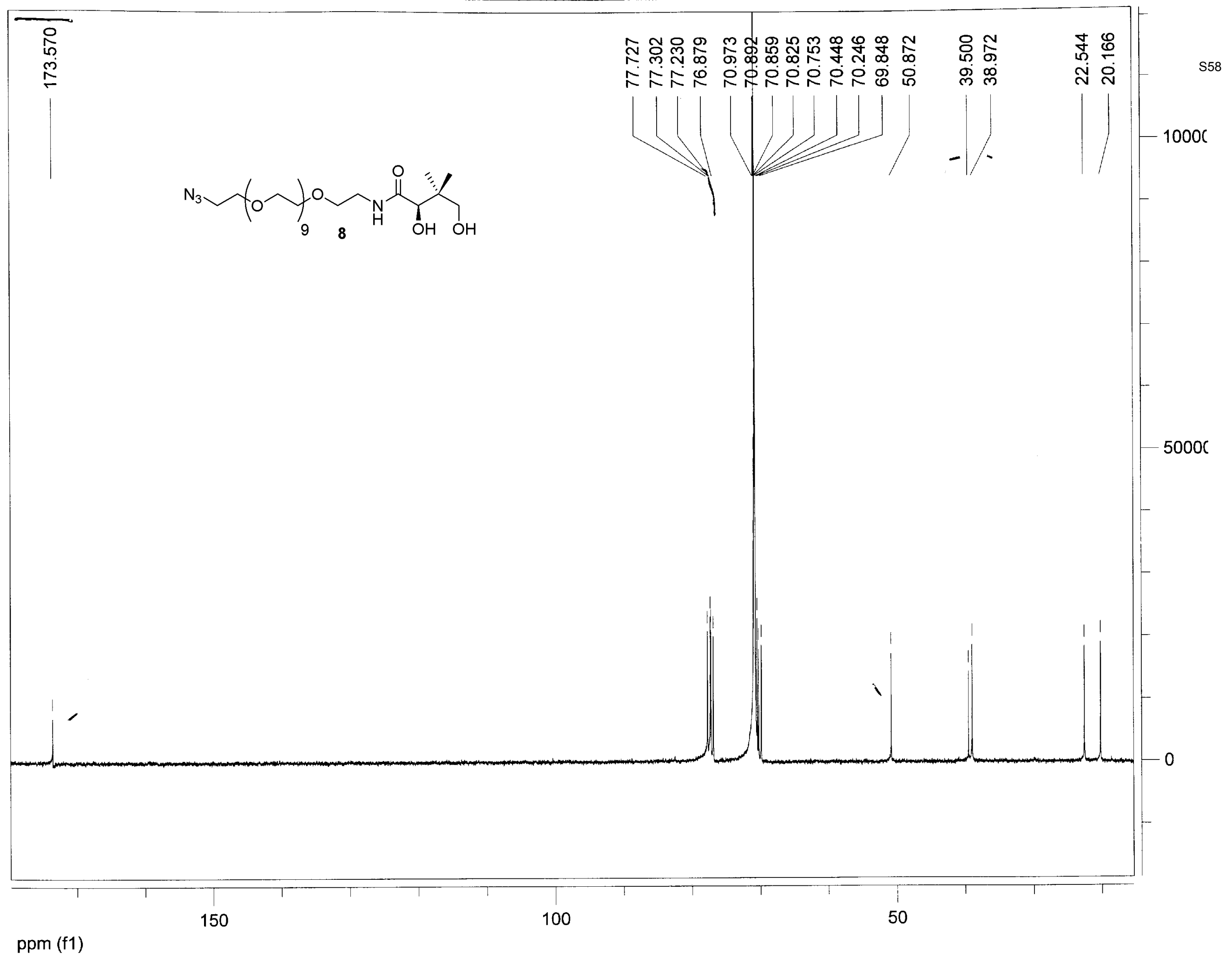


j lm-biot in-propargy lamineconj-pubNMRHNMR-02-07-06

Pulse Sequence: s2pul

Solvent: DMSo

Ambient temperature

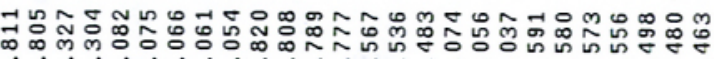

Relax. de lay $1.000 \mathrm{sec}$

Acq. time $1.993 \mathrm{sec}$

Width $6006.0 \mathrm{~Hz}$

OBSERVE H1 400,1252356 MHZ

DATA PROCESSING

Total time $53 \mathrm{~min}, 49 \mathrm{sec}$
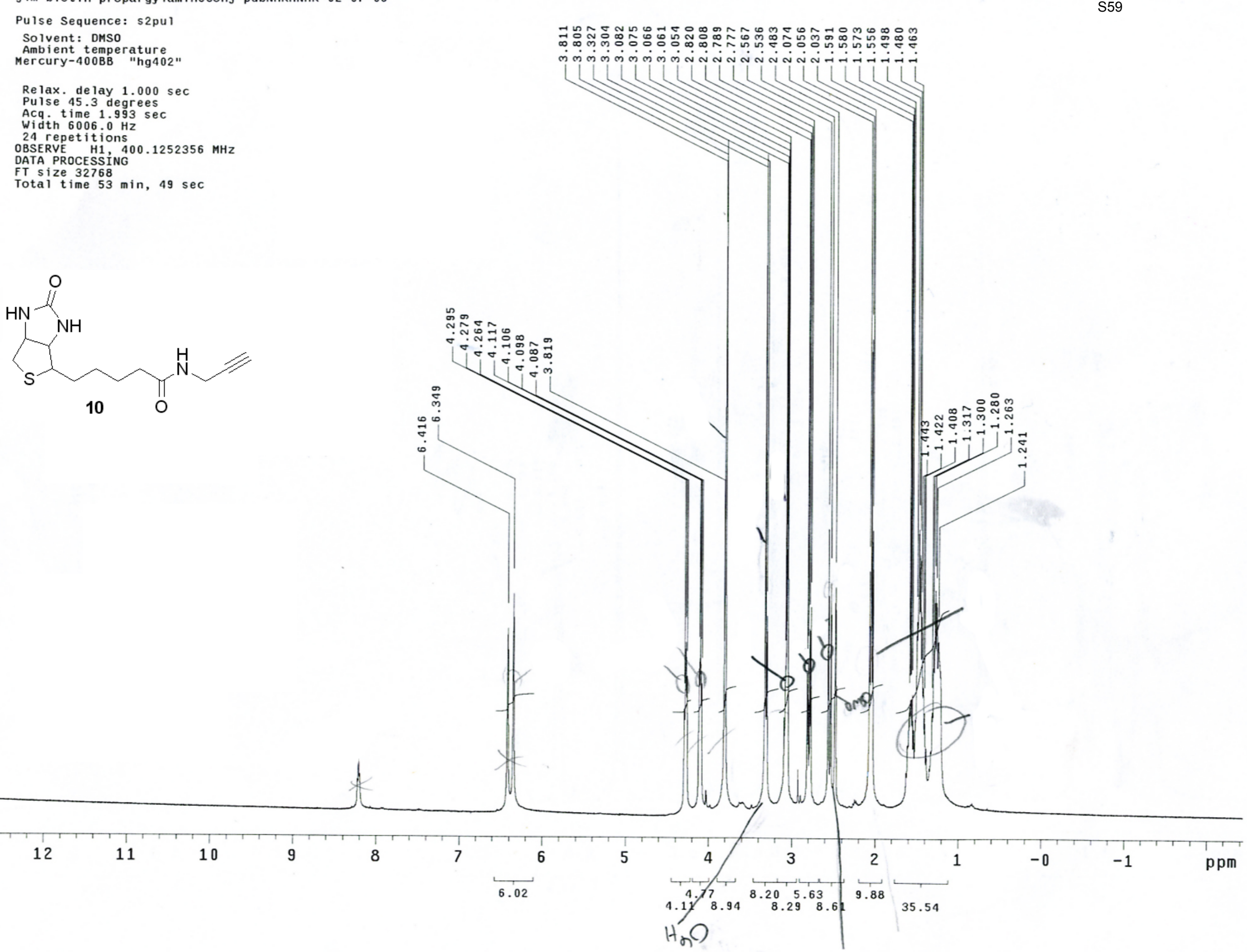
Pulse 53.4 degrees

width $25000.0 \mathrm{~Hz}$

1768 repetitions

OBSERVE C13,100.6115465 MHZ

$08 \mathrm{MHZ}$

cont i nuous iy on

WAL TZ-16 modu Tated

DATA PROCESSING

FT ine broadening $1.0 \mathrm{~Hz}$

rotal time $47 \mathrm{hr}, 17 \mathrm{~min}, 50 \mathrm{sec}$
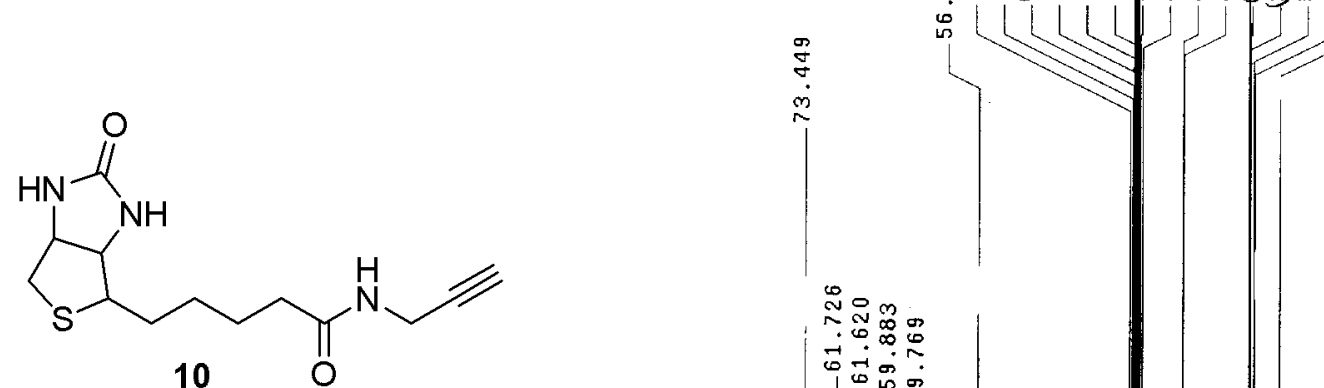

10

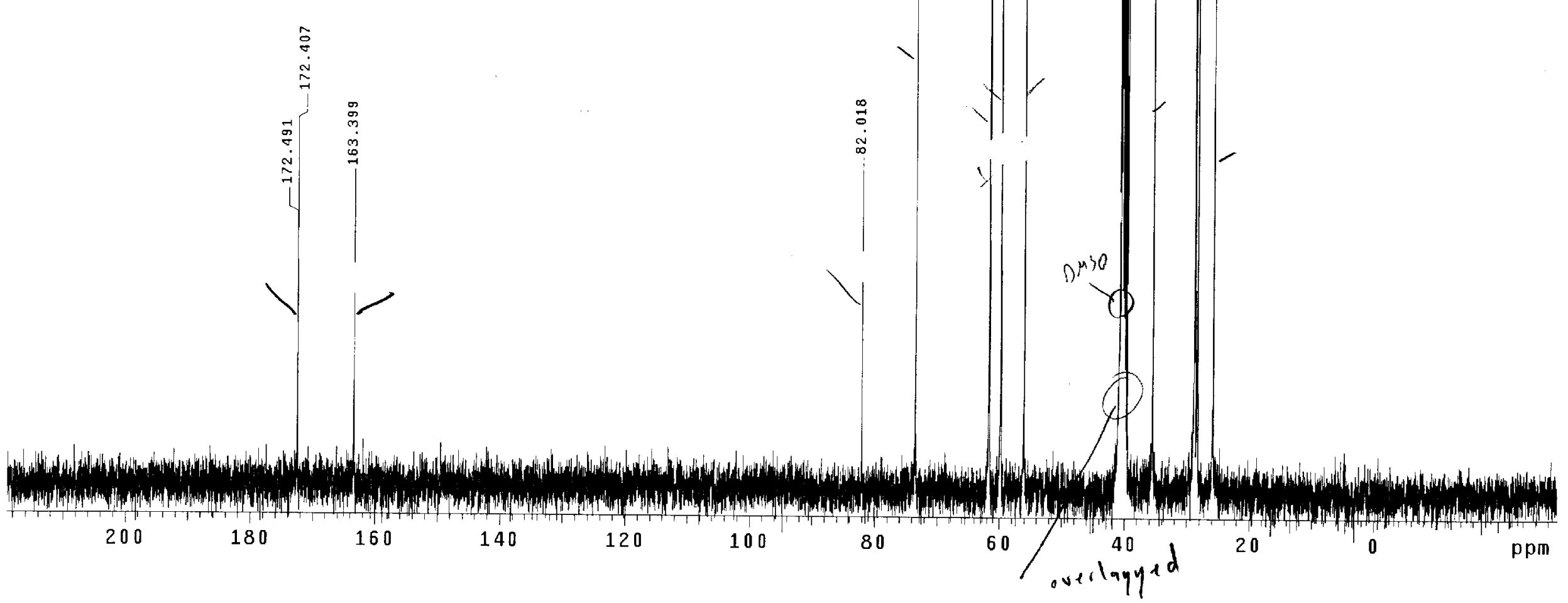


Solvent: DMSO

Temp. $25.0 \mathrm{C}{ }^{2} 298.1 \mathrm{~K}$
UNITY-500 "uns $200 "$

Pulse 51.5 degrees

Acq time 1.892 s
width $8000.0 \mathrm{~Hz}$

120 repetitions

OBSERVE H1,500.3111715 MHZ

DATA PROCESSING
Line broadening $0.3 \mathrm{~Hz}$

FT size 32768 ing $0.3 \mathrm{~Hz}$

Total time $31 \mathrm{~min}, 42 \mathrm{sec}$

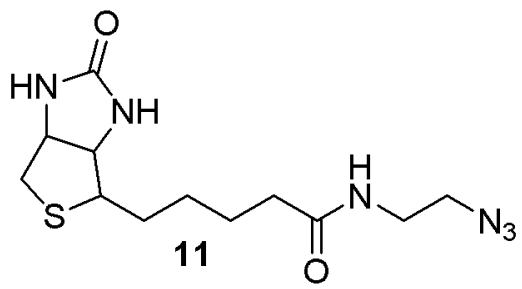

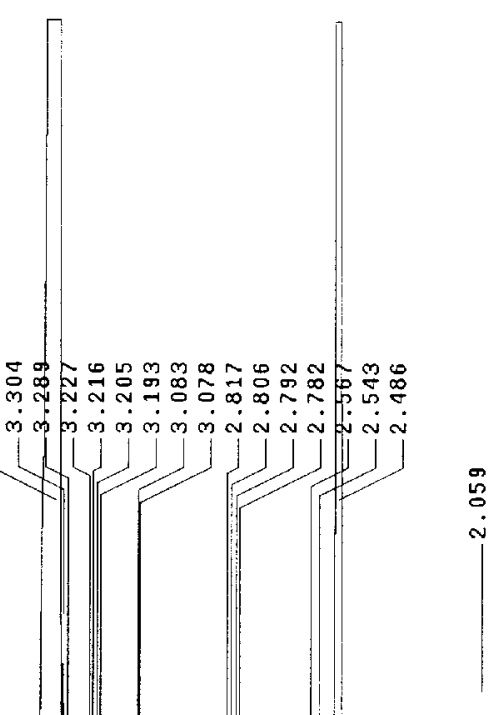
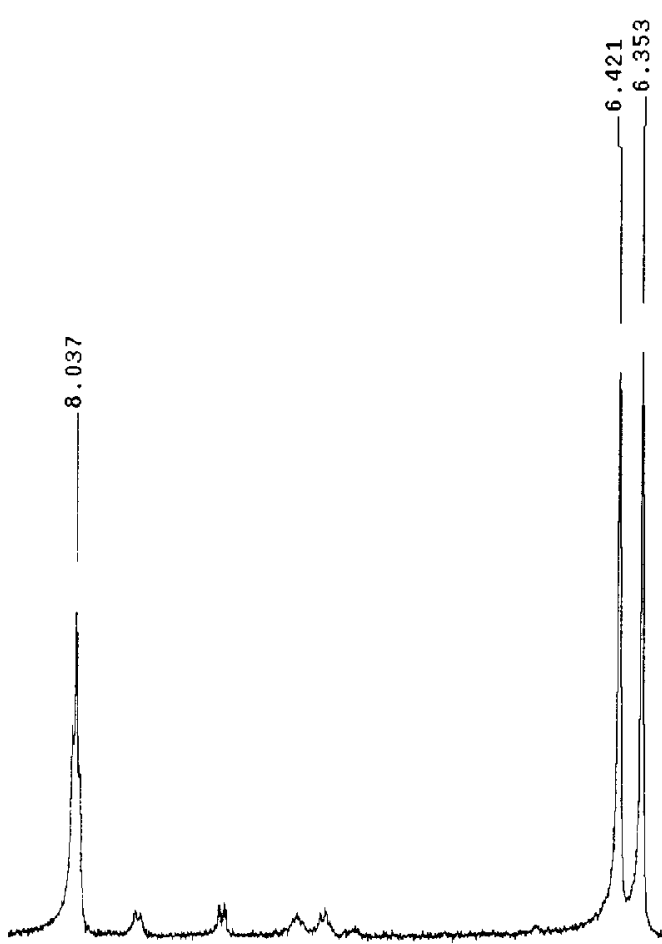

8

4.79

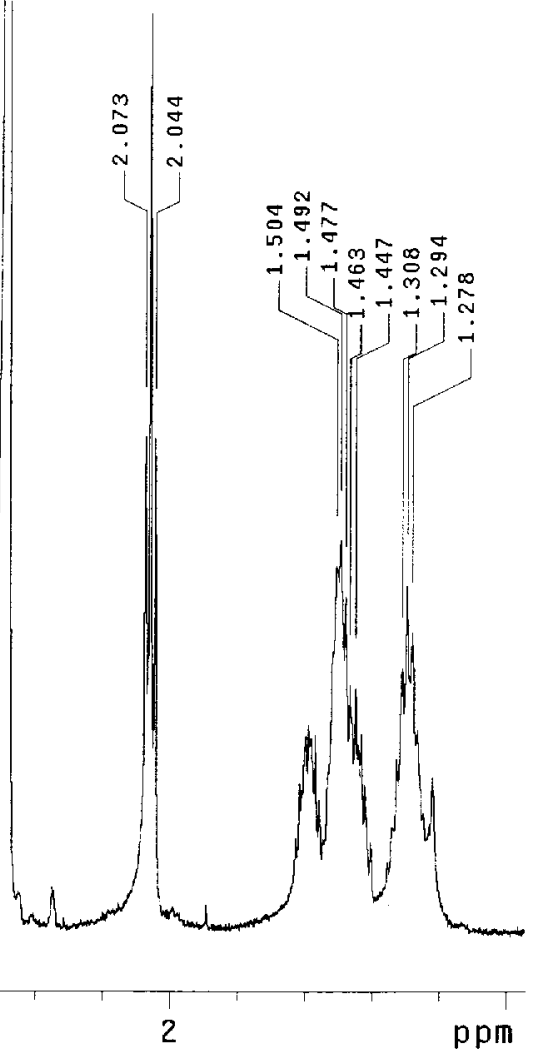


Pulse Sequence: s2pul

Solvent: DMSO

Ambient temperature

mercury-300 "hg300"

Pulse 60.5 degrees

Acq. time $1.815 \mathrm{sec}$

Width $18761.7 \mathrm{~Hz}$

258 repetitions

OBSERVE C13,75.4543874 MHZ

DECOUPLE H1, 300.0784837 MHZ

Power $42 \mathrm{~dB}$

Power 42 dB
cont inuous ly on

WALTZ-16 modulated

DATA PROCESSING

Line broadening $1.0 \mathrm{~Hz}$

FT size 131072

Total time 86minn, 8 éd, $57 \mathrm{sec}$

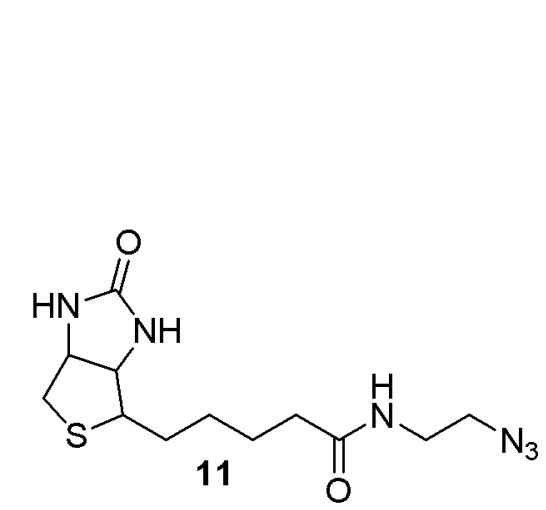

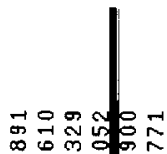

का का का

$m m m$

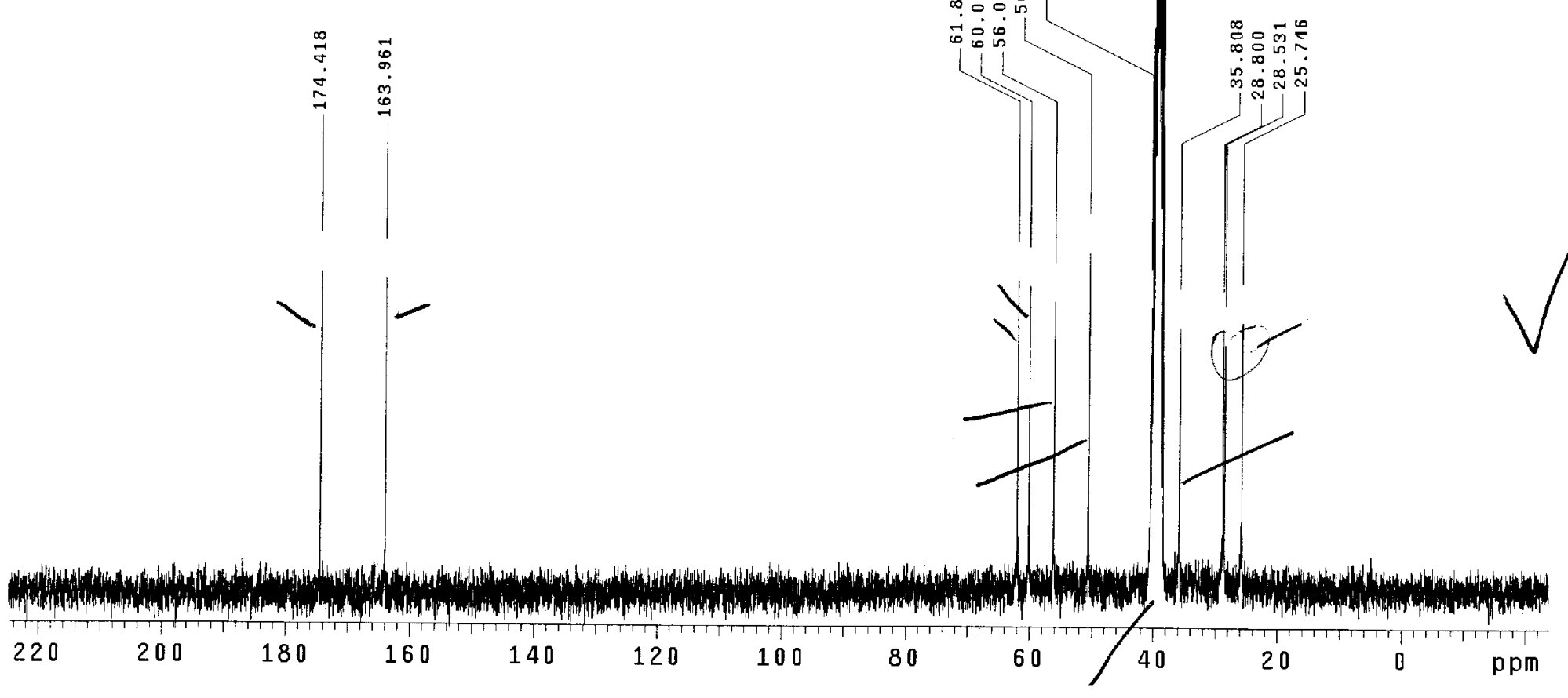


Relax. delay $1.000 \mathrm{sec}$

Pulse 45.3 degree

Acq $t$ ime $1.995 \mathrm{sec}$

Width $4506.5 \mathrm{~Hz}$

56 repet H1 $300.0766925 \mathrm{MHZ}$

DATA PROCESSING

Total time 0 min, 0 sec

280<smiles>CN(C)c1ccc2c(CC(=O)NCCNC(=O)[C@H](O)C(C)(C)CO)cc(=O)oc2c1</smiles>

12
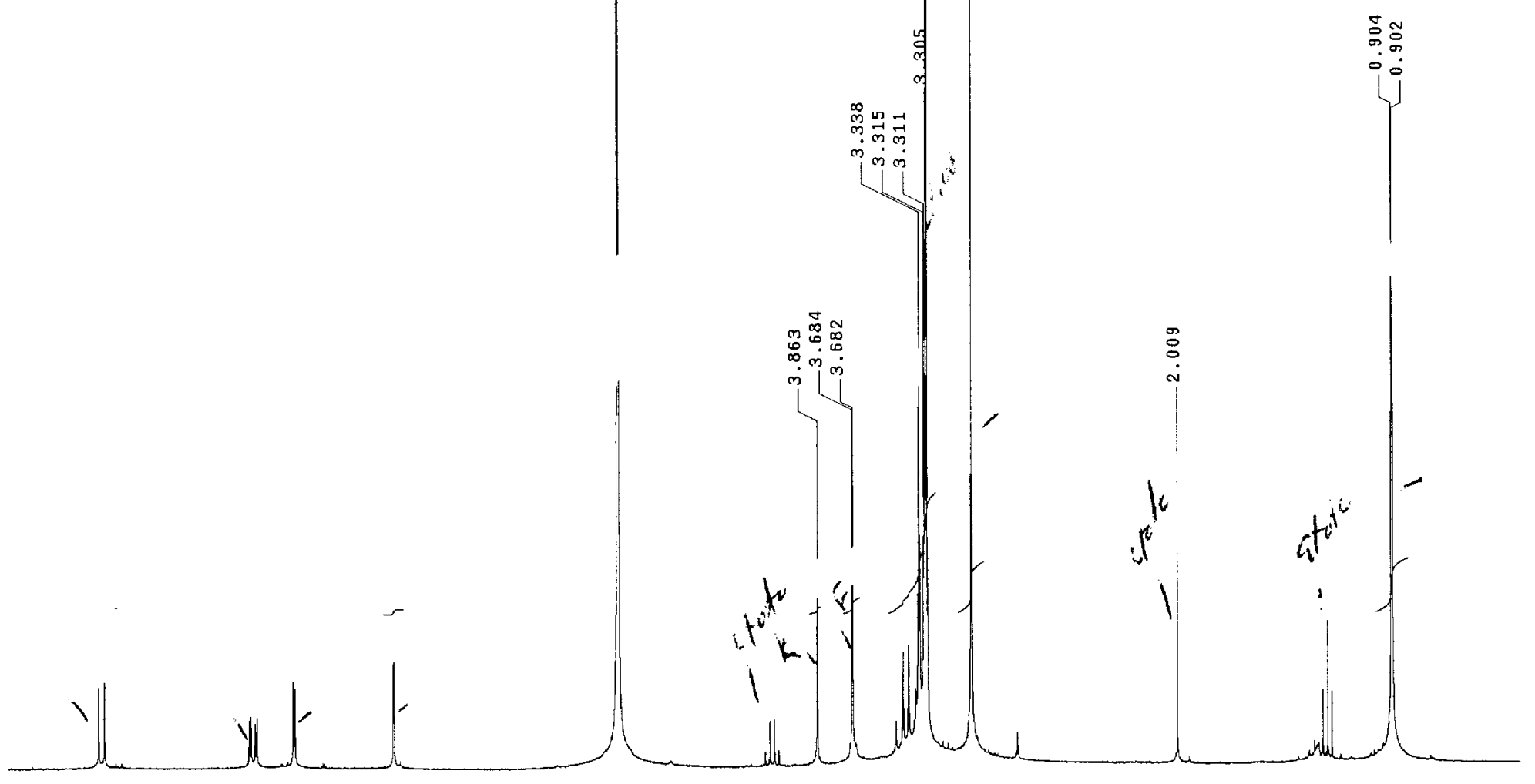

6

4

3

2

ppm

原 4 كיا Tـ 
Ambient temperature

Pulse 60.5 degrees

Acq. time $1.815 \mathrm{sec}$

Width $18761.7 \mathrm{~Hz}$

OBSERVE C13, 75.4543263 MHz

DECOUPLE H1, 300.4543263 MHZ

Power $42 \mathrm{~dB}$

cont inuous ly on

WALTZ-16 modulated
DATA PROCESSING

Line broadening $1.0 \mathrm{~Hz}$

FT size 131072

Total time $0 \mathrm{~min}, 0 \mathrm{sec}$

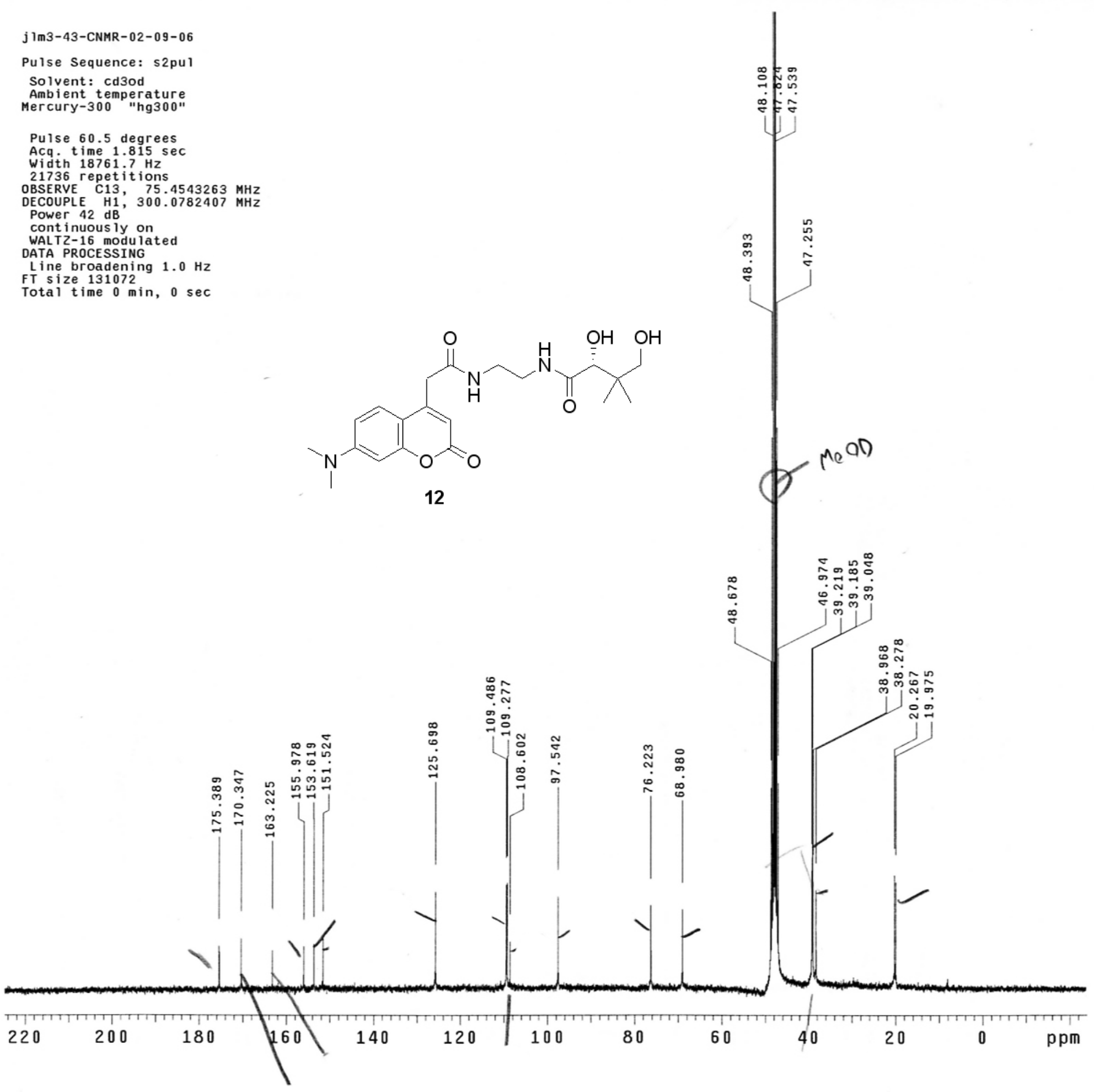




$$
\text { int }
$$


kmc-002-PMPprotecteddansy if i na 1 product -04-16-06

Pulse Sequence: s2pul

Solvent: $\mathrm{CDCl}_{3}$

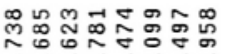

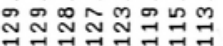

Pulse 53.4 degrees

Width $25000.0 \mathrm{~Hz}$

2688 repetitions

$.6110686 \mathrm{MH}$

cont i nuous ly on

WALTZ-16 modulate

Line broadening $1.0 \mathrm{~Hz}$

$-N$

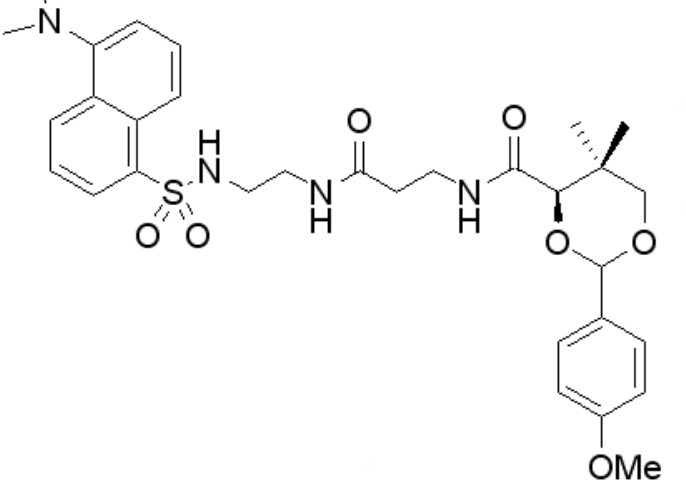

$\overbrace{0}^{1}$

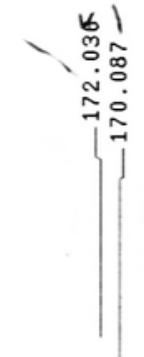

Me
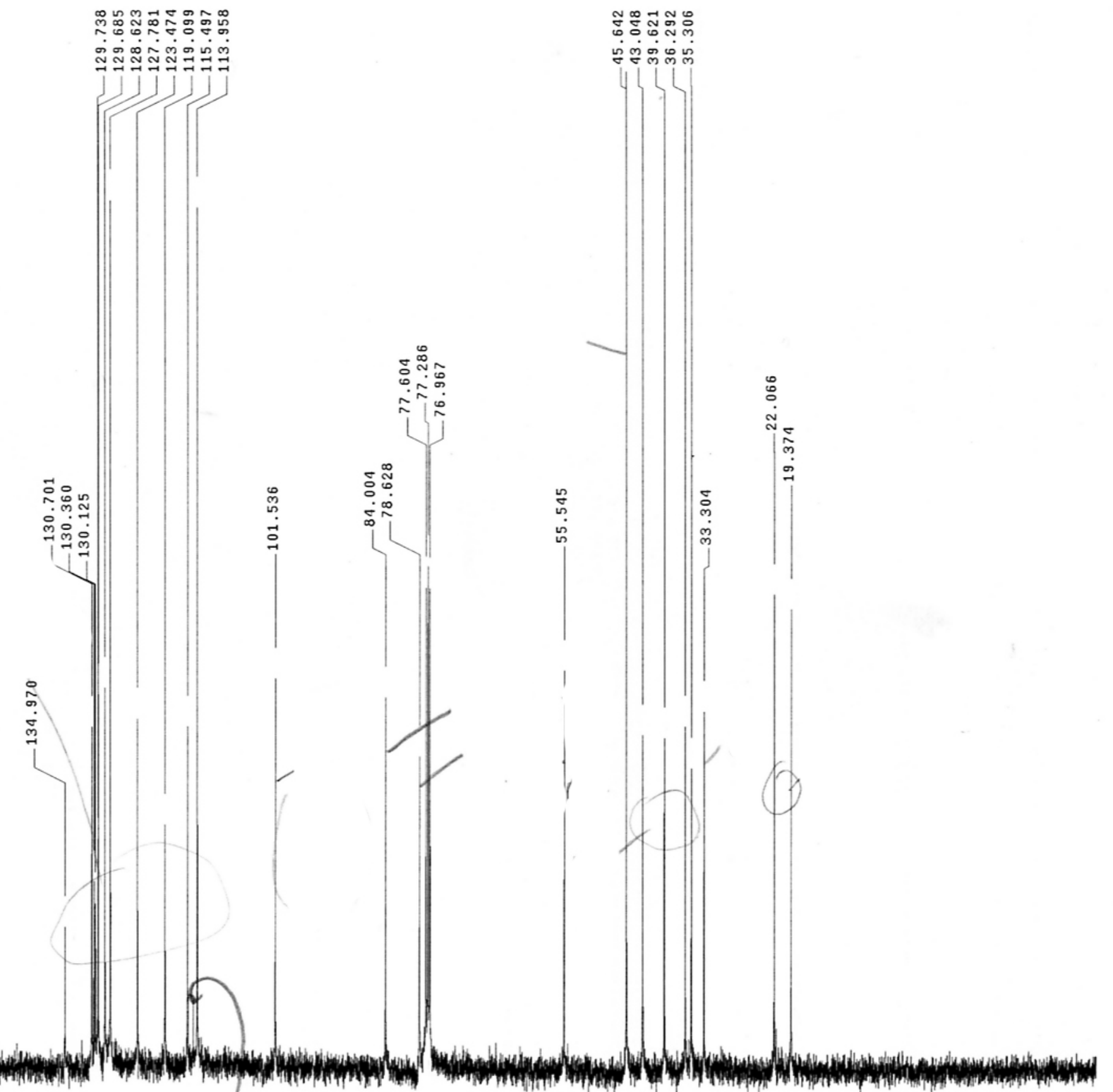

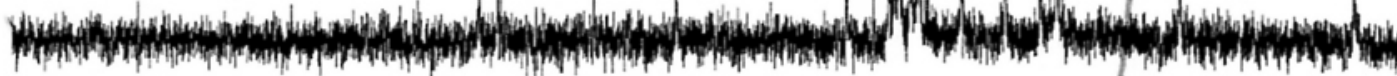

200

180

160

140

120

100

80

60

40

20 
STANDARD PROTON PARAMETERS

Pulse Sequence: s2pul

Solvent: DMSO

Temp. $25.0 \mathrm{C} / 298.1 \mathrm{~K}$
UNITY-500

Pulse 51.5 degrees

Width $8000.0 \mathrm{~Hz}$

96 repetitions

OBSTEN H1, 500.3111715 MHz

等

FT size 32768 mín

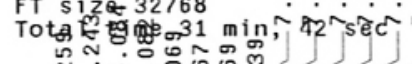

N.

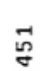

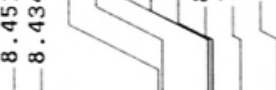

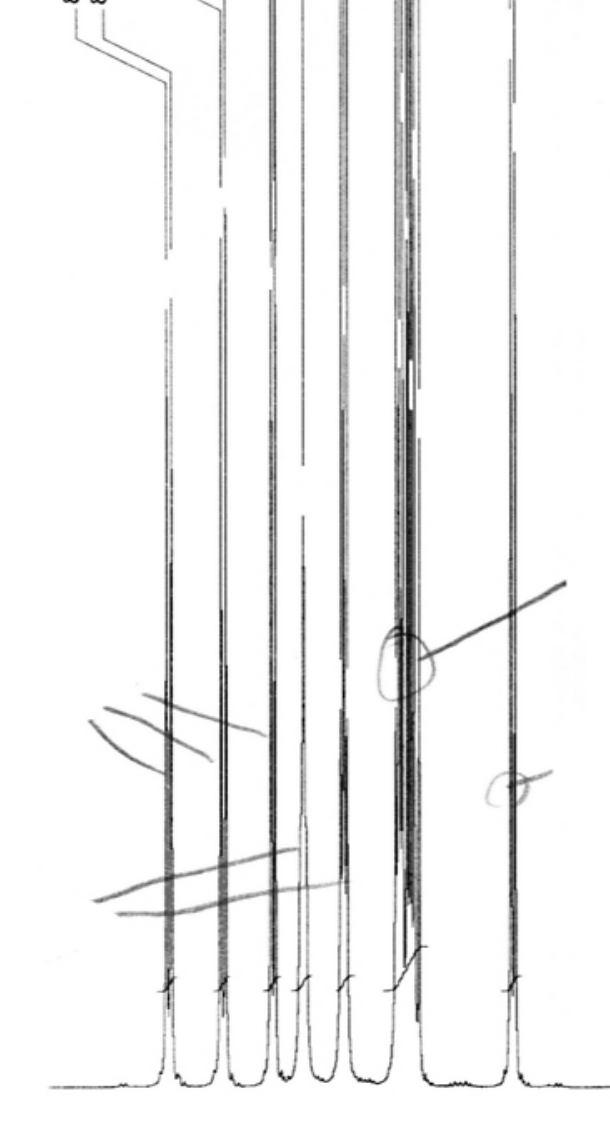

N

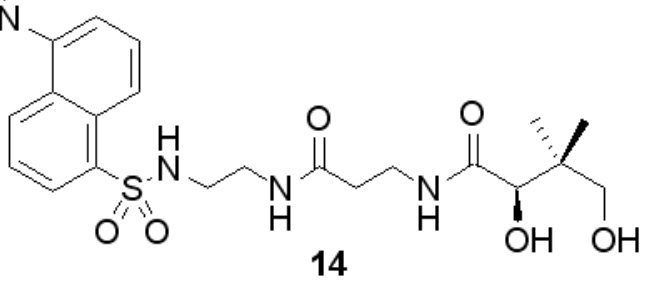

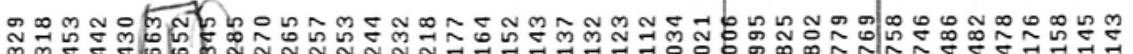

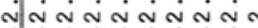


Pulse Sequence: s2put

Solvent: $\operatorname{CoC13}$
Ambient temperature
Mercury-300 "hg300"

Pulse 60.5 degrees

Acq. time 1.815 se

976 repetitions

OBSERVE C13, $75.4540290 \mathrm{MHZ}$

DECOUPLE H1, $300.0770584 \mathrm{MHZ}$

Power 42 dB

WALTZ-16 modulated

DATA PROCESSING

TT ne broadening $1.0 \mathrm{~Hz}$

Total time $6 \mathrm{hr}, 36 \mathrm{~min}, 23 \mathrm{sec}$

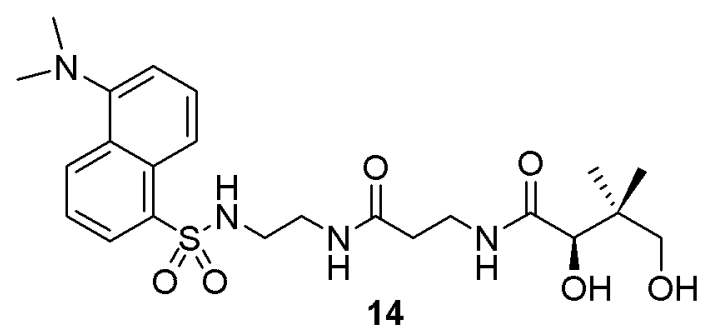

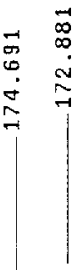

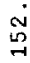
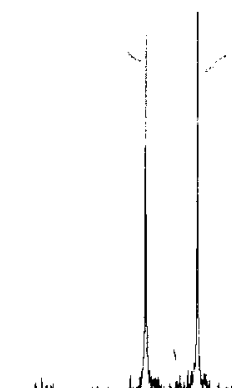

14

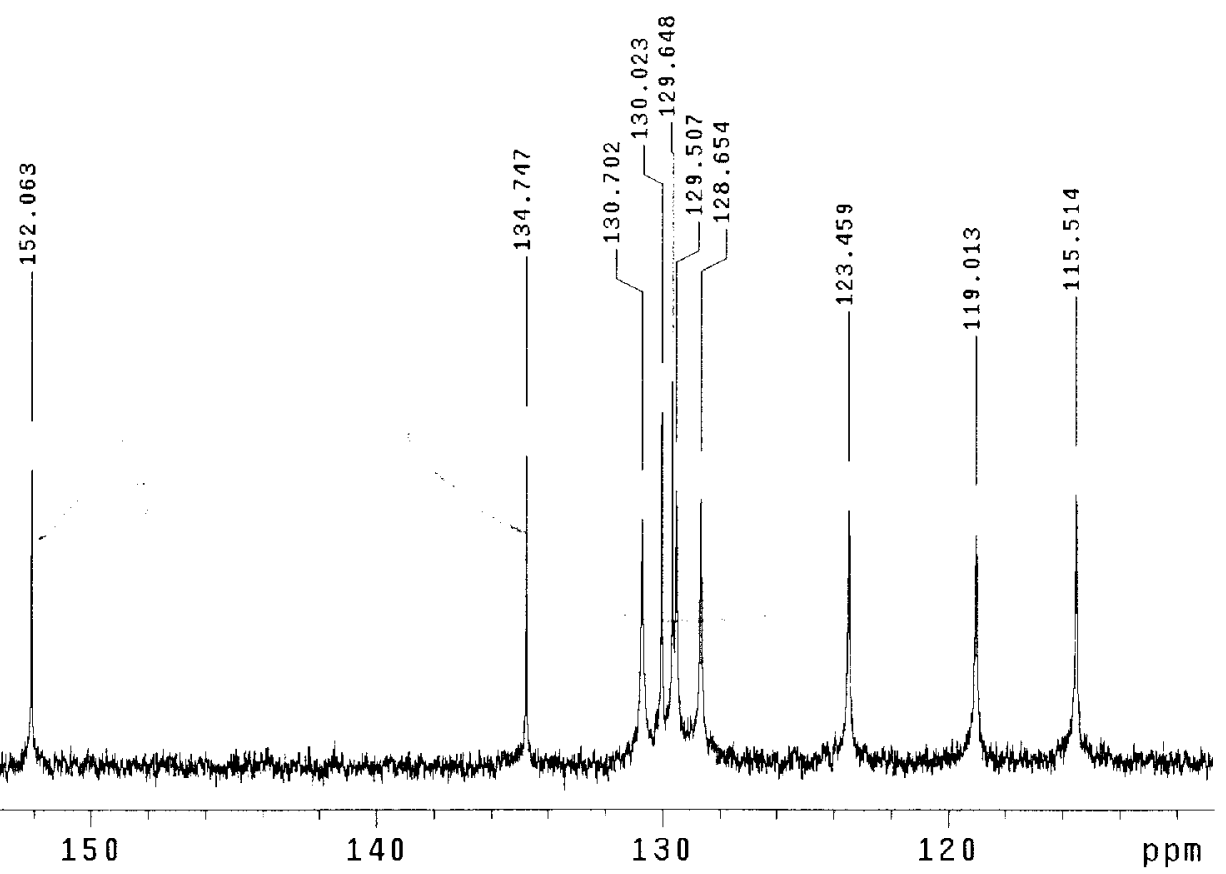




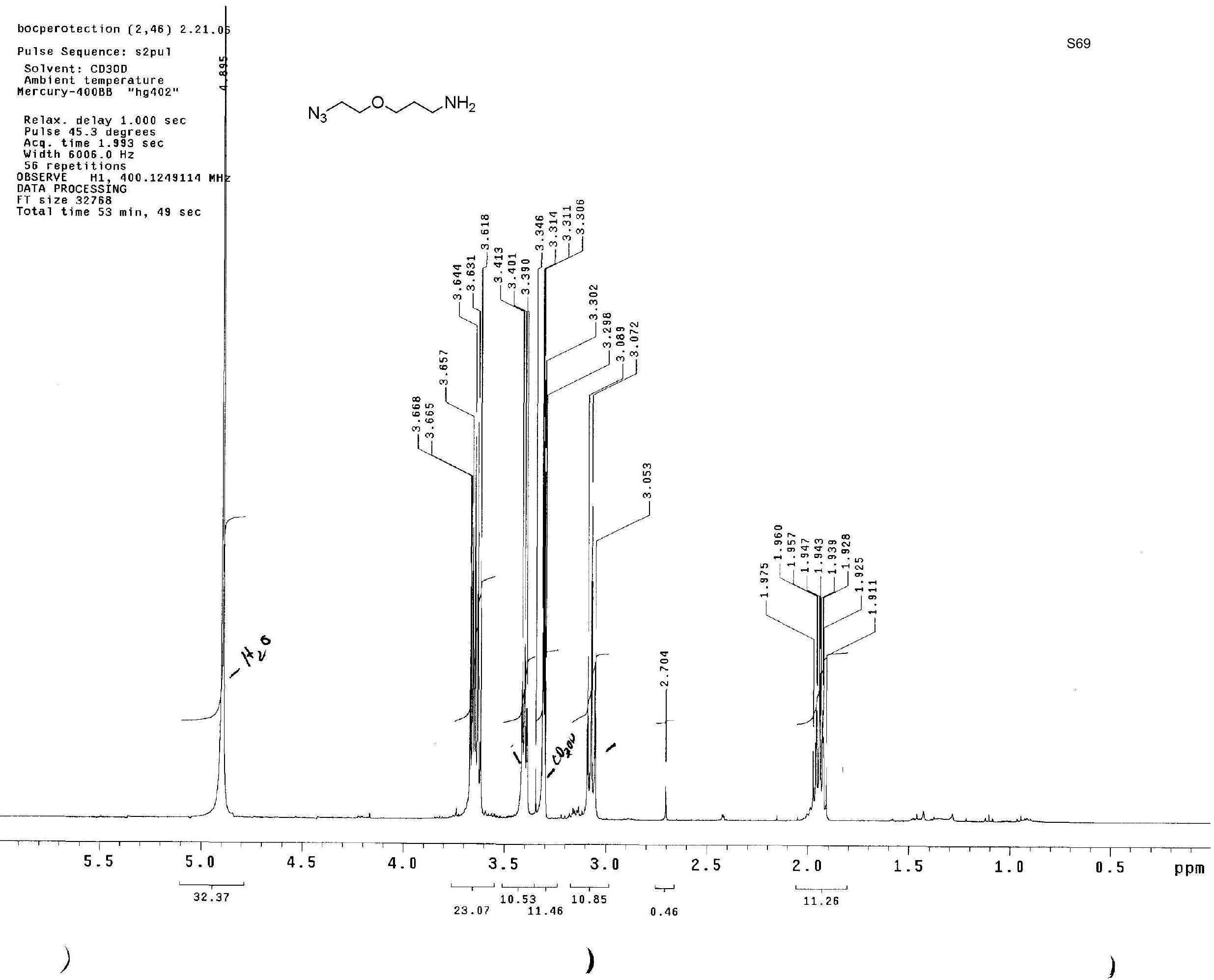


Pulse Sequence:
Solvent: CD30D

Solvent: CD3od
Ambient temperature
Mercury-400B "ng402"

Putse 53.4 degrees

Acq time $1.199 \mathrm{sec}$

titions

OBSERVE C13, 100.6114650 MHZ

DECOUPLE 38 H

cont inuously on

WALTZ-16 modulate

DATA PROCESSING

FT size 65536 ing $1.0 \mathrm{~Hz}$

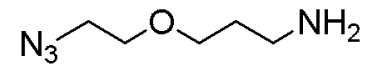

FT size 65536 min, $1 \mathrm{sec}$

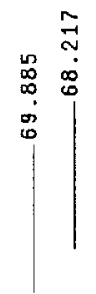

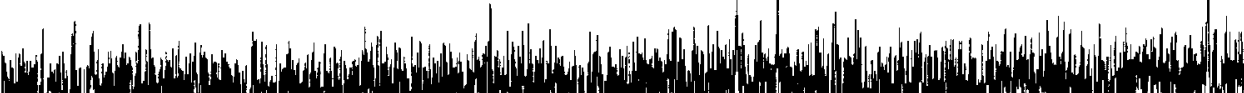

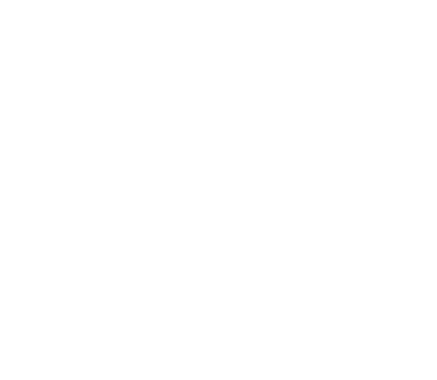

$\underset{0}{3}$

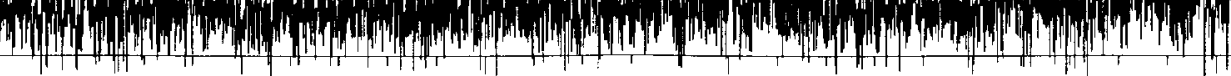

90

80

70

60

50

40

30

20

ppm 
coumarin-azide $(2,49) 2.23 .05$

Pulse Sequence: s2pul

Solvent: $\operatorname{CDC} 13$

UNITY-500 "un500" $258.1 \mathrm{~K}$

Pulse 51.5 degrees

Acq. time $1.892 \mathrm{se}$
width $8000.0 \mathrm{~Hz}$

240 repetitions Line broadening $0.3 \mathrm{~Hz}$

FT size 32768
Total time $31 \mathrm{~min}, 42 \mathrm{sec}$
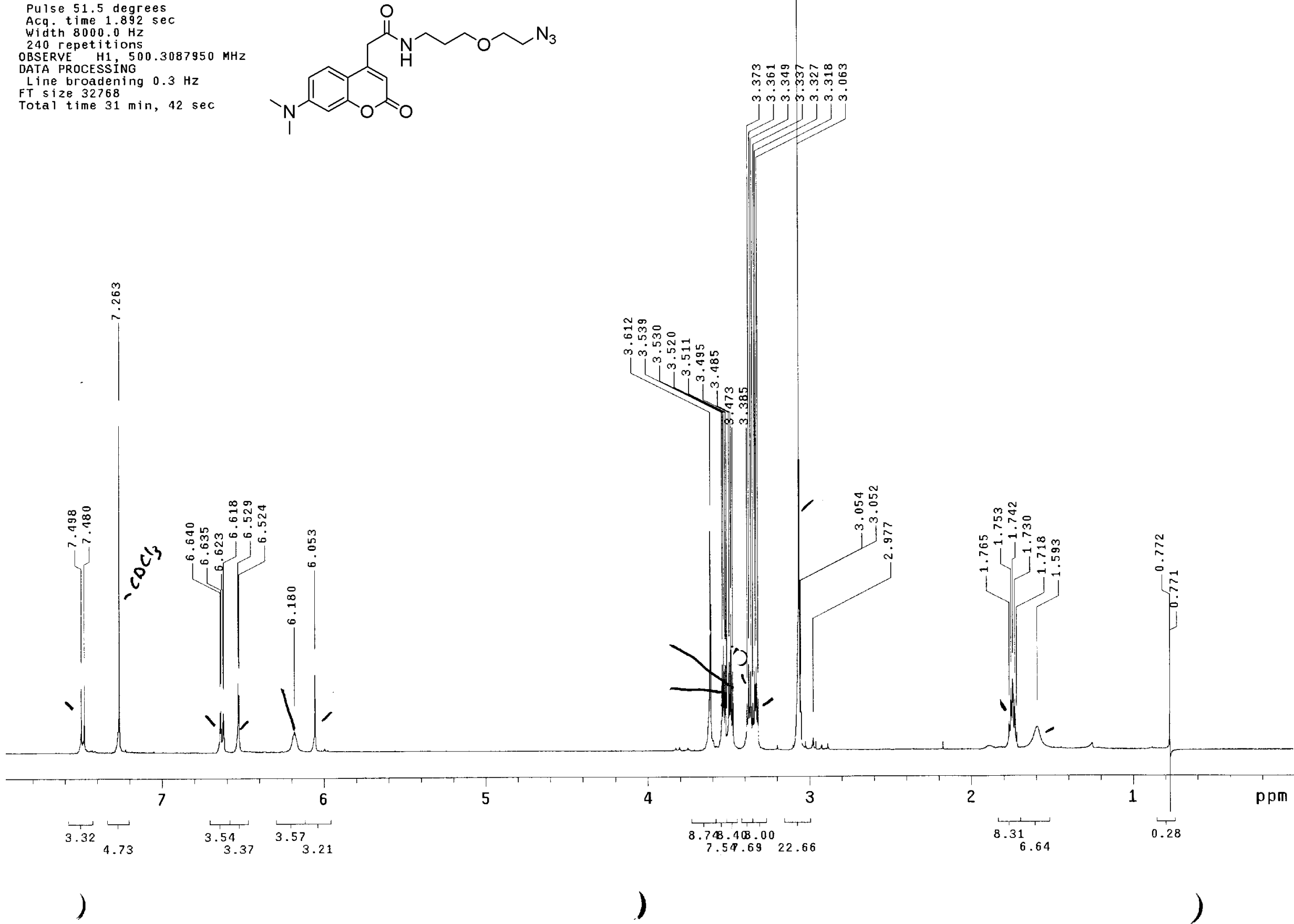

7.54 .6922 .66 
cou-azide $(2,49) 3.7 .06$

Pulse Sequence: $52 \mathrm{put}$

Solvent: $\operatorname{CDC} 13$

Ambient temperature
Mercury-300 "hg300"

Pulse 60.5 degrees

Acq. time $1.815 \mathrm{se}$

OBSERVE C13, 75.4540290 MHZ

DECOUPLE H1, 300.0770584 MHZ

Power $42 \mathrm{~dB}$

cont inuous $1 \mathrm{y}$ on

DATA PROCESSING

Line broadening $1.0 \mathrm{~Hz}$

Total time $5 \mathrm{hr}, 52 \mathrm{~min}, 1 \mathrm{sec}$
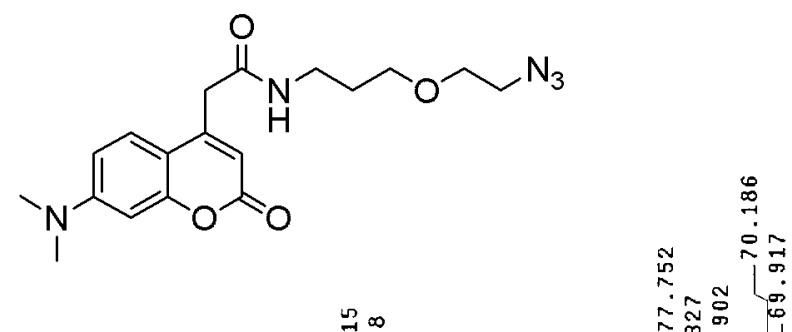

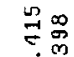

:

离

离

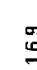

$\underset{0}{7}$

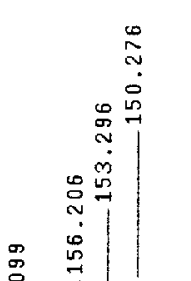

$\underset{0}{0}$

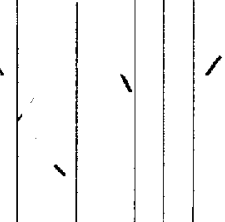

$$
\underset{0}{2}
$$

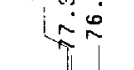

交

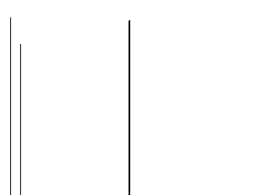

$\stackrel{\rho}{\rightarrow \infty}$

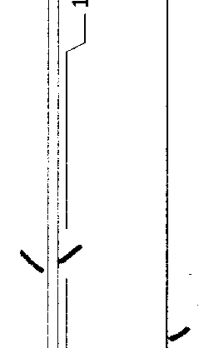

1600 $18761.7 \mathrm{~Hz}$

WALT -16 modillated

$\overrightarrow{0}$

$\&$

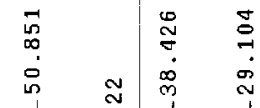

$\underset{N}{*} \int_{0}^{\infty} \stackrel{\infty}{\infty}$

i

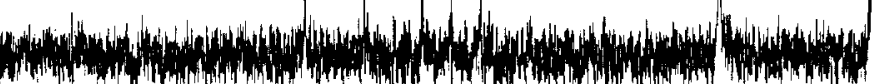

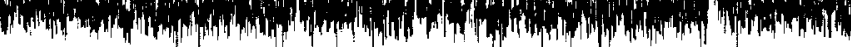

180

160

140

120

100

80

60

40

20

ppm 
Relax. de lay $1.000 \mathrm{sec}$

Pulse 45.3 degrees

Acq. time 1.993 sec

Width $6006.0 \mathrm{~Hz}$

OBSERVE H1, 400.1249114 MHZ

FT Size 32768

Total time 0 min, $0 \mathrm{sec}$

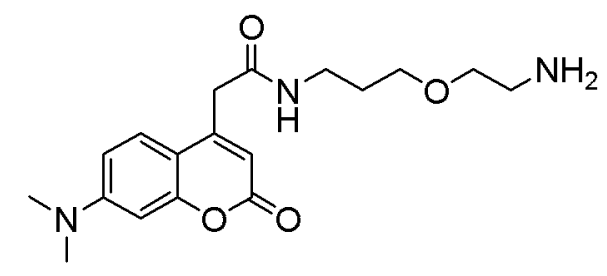

8

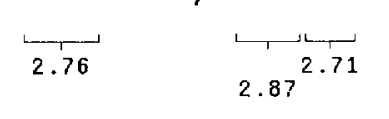

2.87

6

6

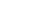


freeamine/triethamine $(2,63) 3.27 .06$

Puise Sequence: $52 \mathrm{pu} 1$

Solvent: CD30D

Ambient temperature

Mercury-300 "hg300"

Pulse 60.5 degrees

Acq $\mathrm{time} 1.815 \mathrm{sec}$

Width $18761.7 \mathrm{~Hz}$

2464 repetitions

OBSERVE C13, 75.4543263 MHZ

DECOUPLE H1; 300.0782407 MH

Power $42 \mathrm{~dB}$

continuous iy on

WALTZ-16 modulated

DATA PROCESSING

Line broadening $1.0 \mathrm{~Hz}$

FT size 131072

Total time 0 min, 0 sec
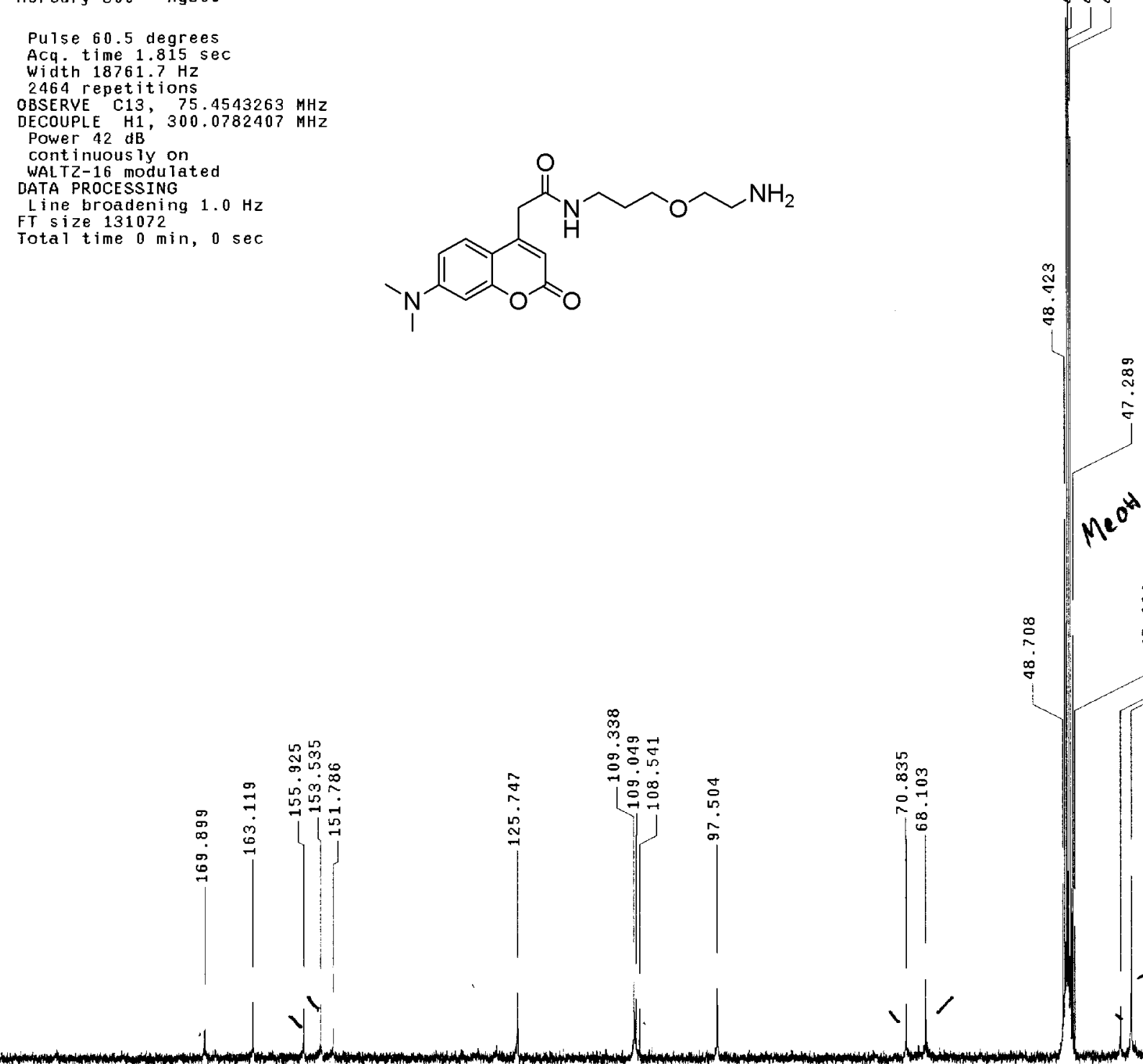

\section{두용} ig 
final product $\operatorname{aA}(2,65) 4.3 .06$

Pulse Sequence: s2pul

Solvent: $\operatorname{CDC} 13$

Ambient temperature
Mercury-400B "hg $402 "$

Relax. delay 1.000 sec

Pulse 45.3 degrees

Acq. time $1.993 \mathrm{sec}$

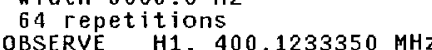

DATA PROCESSING

Total time 0 min, 0 sec
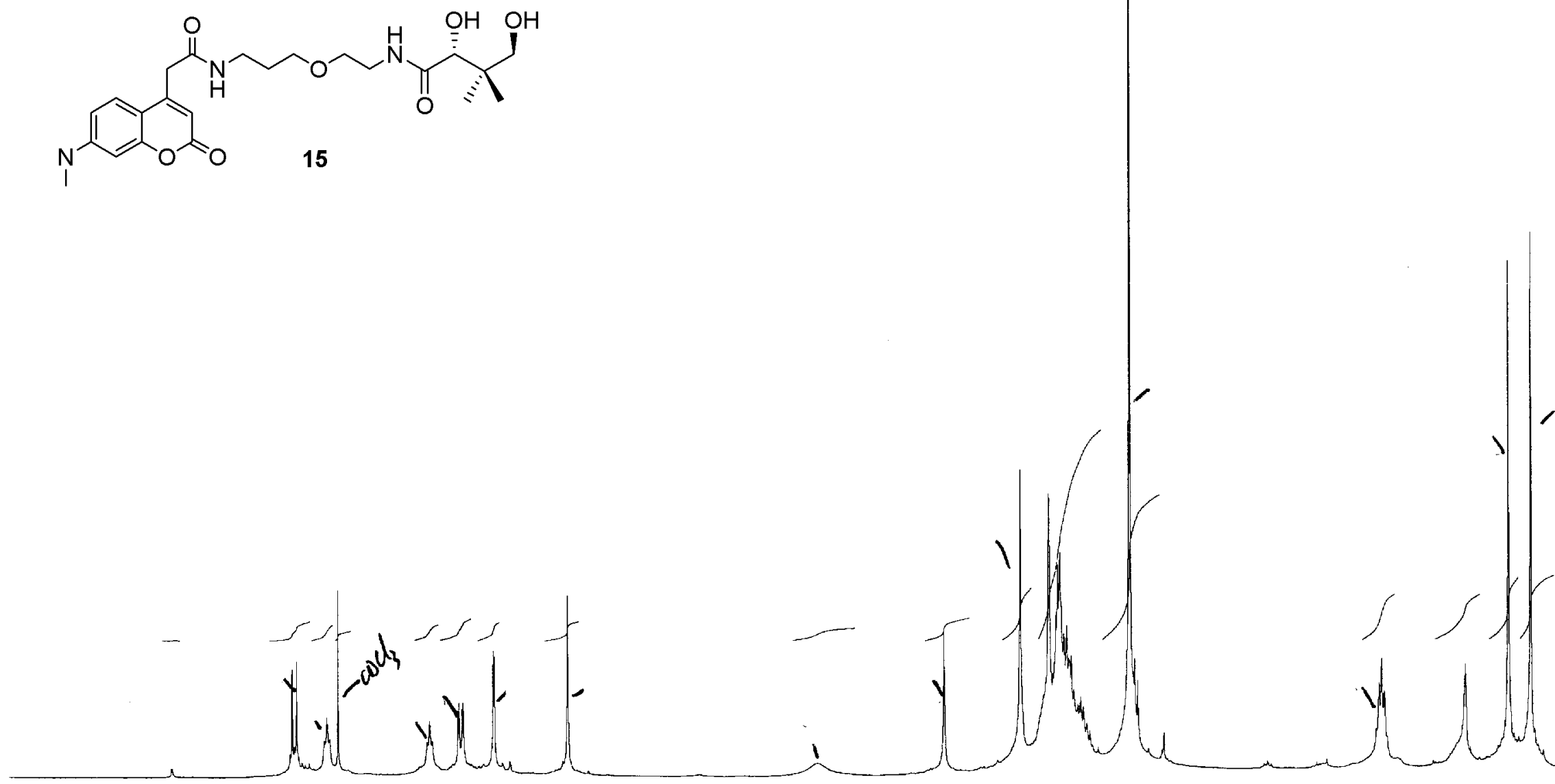
final prod $A(2,65) 4.4 .06$

Pulse Sequence: s2pul

Solvent: $\operatorname{CDC} 13$

Ambient temperature
Mercury-400B B "hg $402 "$

Pulse 53.4 degrees

Acq. time $1.199 \mathrm{sec}$

2256 $25000.0 \mathrm{~Hz}$

OBSERVE C13, 100.6110686 MHZ

DECOUPLE H1, 400.1253602 MHZ

Power $38 \mathrm{~dB}$

cont inuously on

DATA PROCESSING

Line broadening $1.0 \mathrm{~Hz}$

FT size 65536

Total time $0 \mathrm{~min}, 0 \mathrm{sec}$
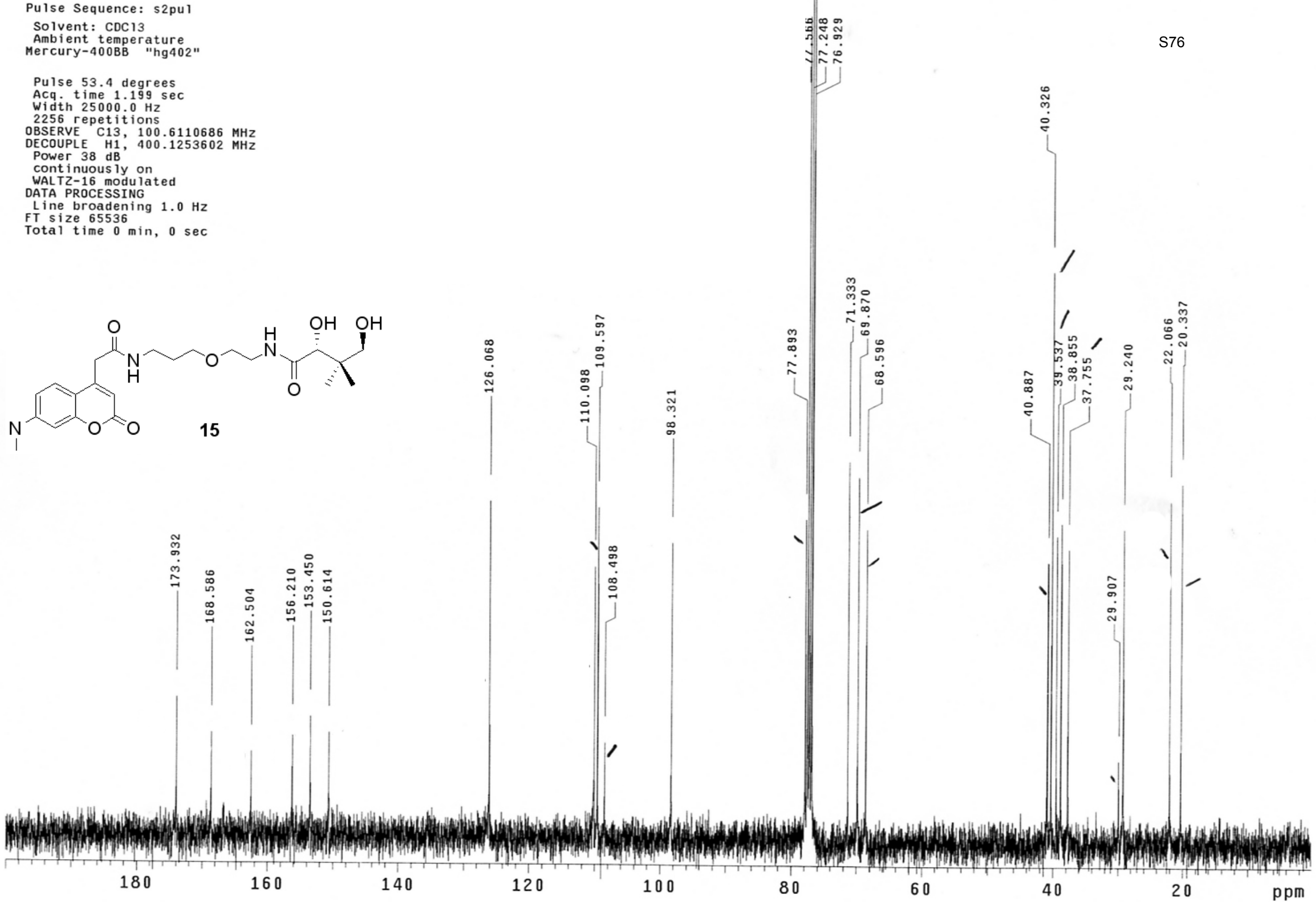
staudinger $(2,48) 2.22 .06$ Pulse Sequence: s2pul Solvent: $C D 30 D$

Ambient temperature Mercury-300 "hg300"

Relax. delay $1.000 \mathrm{sec}$ Pulse 45.3 degrees

Acq. time $1.995 \mathrm{sec}$

Width $4506.5 \mathrm{~Hz}$

64 repetitions

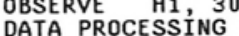

FT Size 32768

Total time $0 \mathrm{~min}, 0 \mathrm{sec}$<smiles>CC(C)(C)OC(=O)NCCCOCCN</smiles>

$\mathrm{H}_{2} \mathrm{~N} \curvearrowright \mathrm{O}$
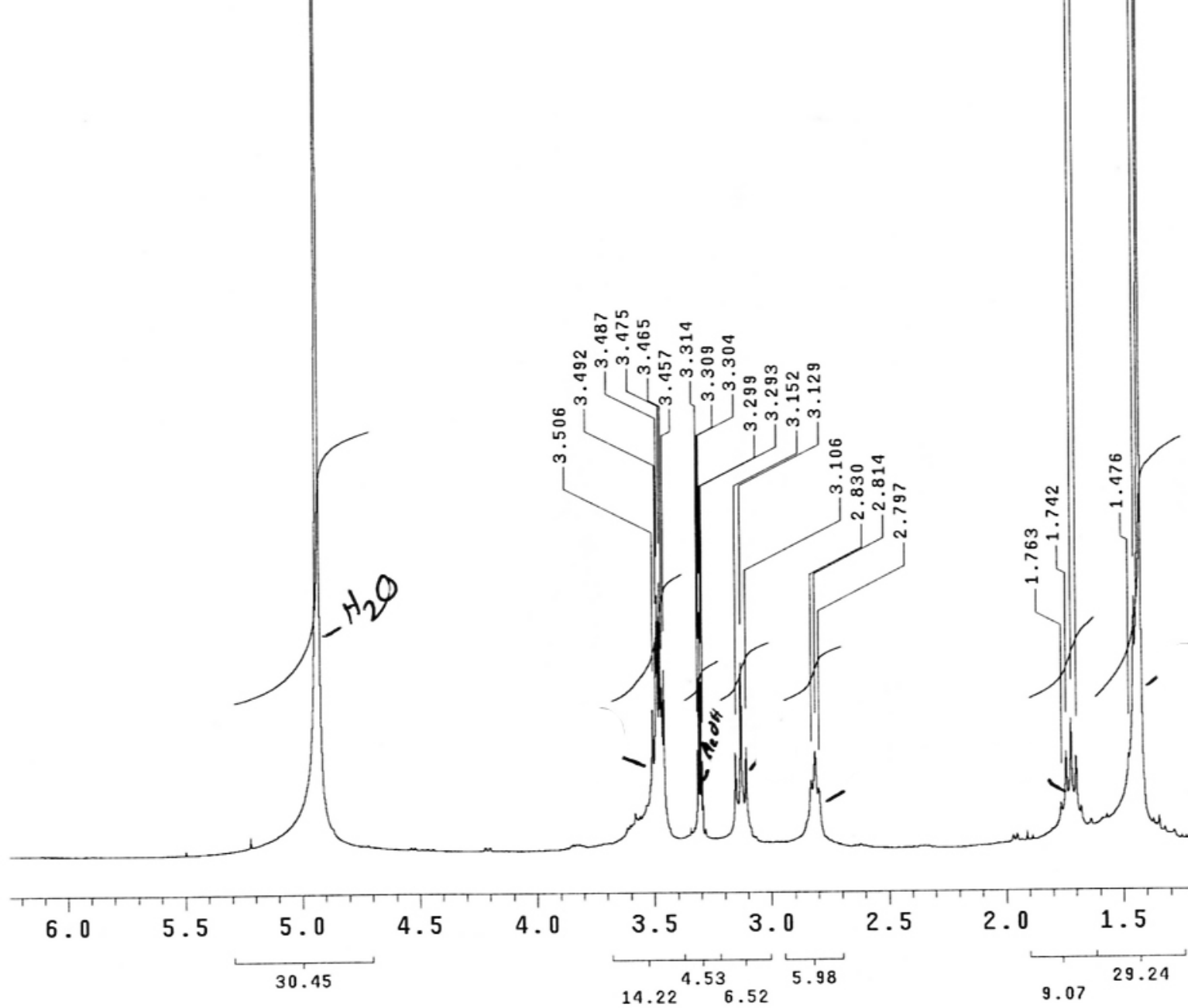

2.5

2.0

1.5

1.0

0.5

ppm 
Acq. time $1.199 \mathrm{sec}$

1424 repetitions

OBSERVE C13, $100.6114650 \mathrm{MHZ}$

DECOUPLE H1, 400.1269367 MHZ

Power $38 \mathrm{~dB}$

cont

DATA PROCESSING

Line broadening $1.0 \mathrm{~Hz}$

Total time $0 \mathrm{~min}, 0 \mathrm{sec}$
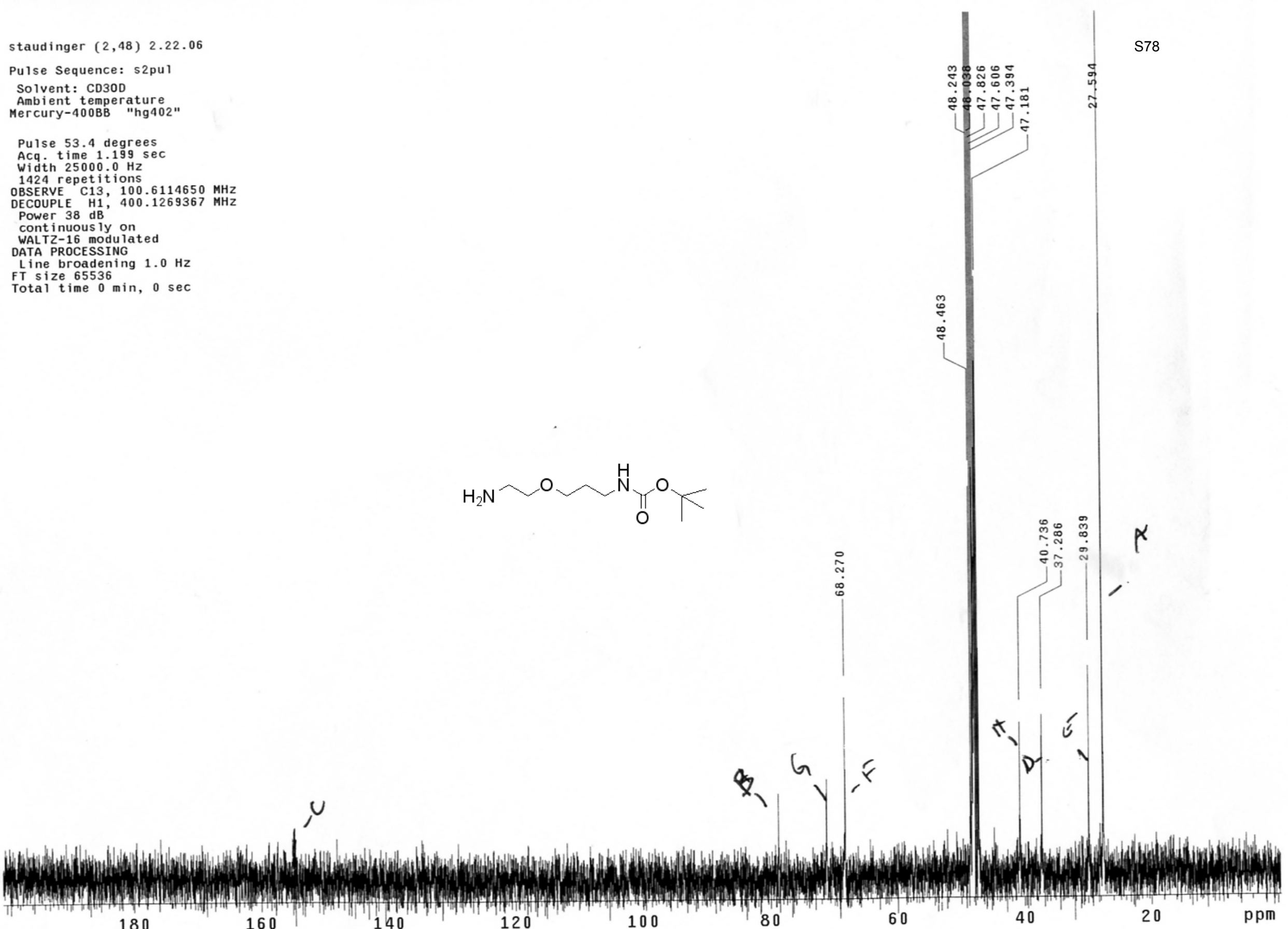
boc-coumarin-after 2nd prep $(2,50) 4.5 .06$

\section{Pulse Sequence: s2pul}

Solvent: Acetone

Ambient temperature
Mercury-400BB "hg402"

Relax. delay $1.000 \mathrm{sec}$

Pulse 45.3 degrees

Acq. time $1.993 \mathrm{~s}$

Width $6006.0 \mathrm{~Hz}$

OBSERVE H1, 400.1254116 MHz

DATA PROCESSING

Total time $53 \mathrm{~min}, 49 \mathrm{sec}$
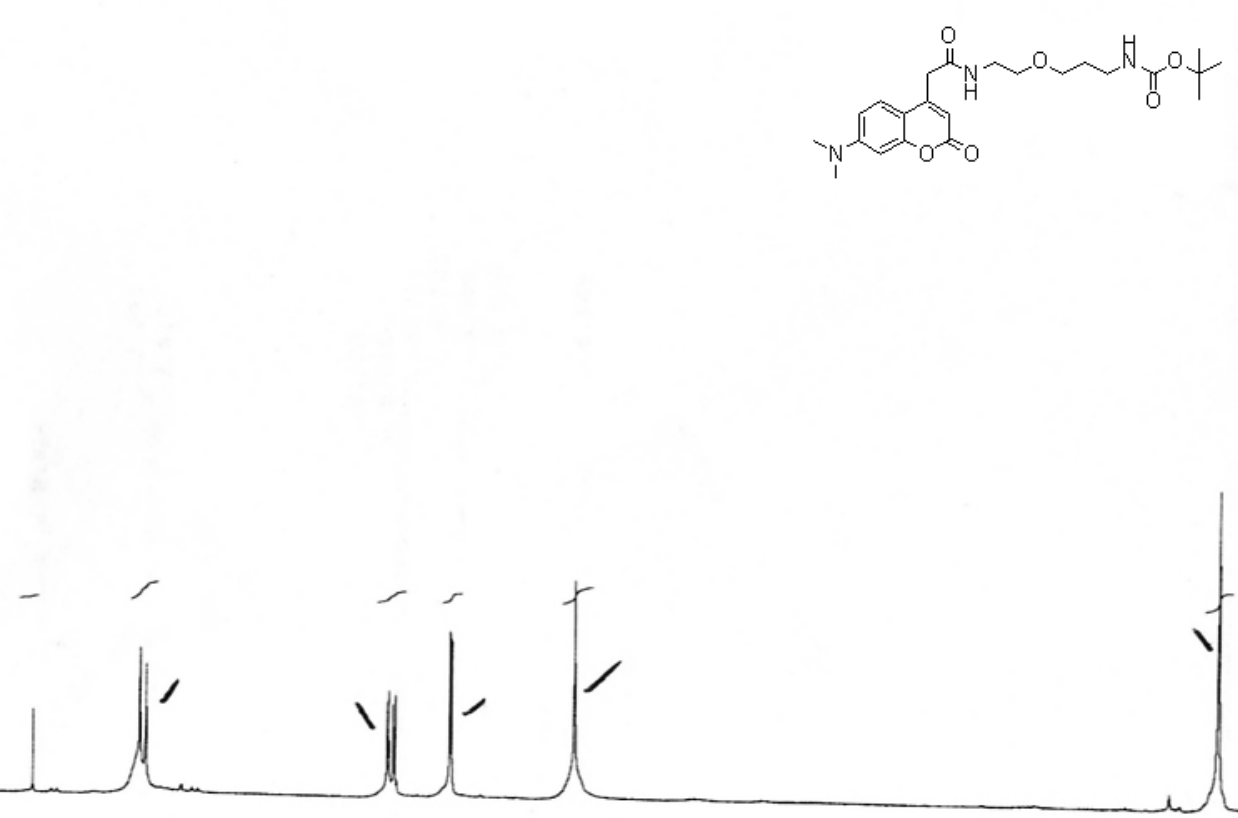

8
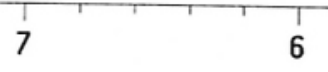

0.24

$\stackrel{4}{0.90}$

$\sqrt{1.39}$

5

$5+1$

4

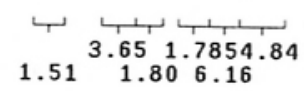

$1.51 \begin{aligned} & 3.651 .7854 .84 \\ & 1.806 .16\end{aligned}$

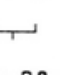


Pulse 53.4 degrees

width $25000.0 \mathrm{~Hz}$

57968 repetition

OBSERVE C13, $100.6110686 \mathrm{MH}$

DECOUPLE H1, 400.1253602 MH

Power 38 dB

WALTZ-16 ly on

DATA PROCESSING

Line broadening $1.0 \mathrm{~Hz}$

FT size 65536
Total time $47 \mathrm{hr}, 17 \mathrm{~min}, 50 \mathrm{sec}$
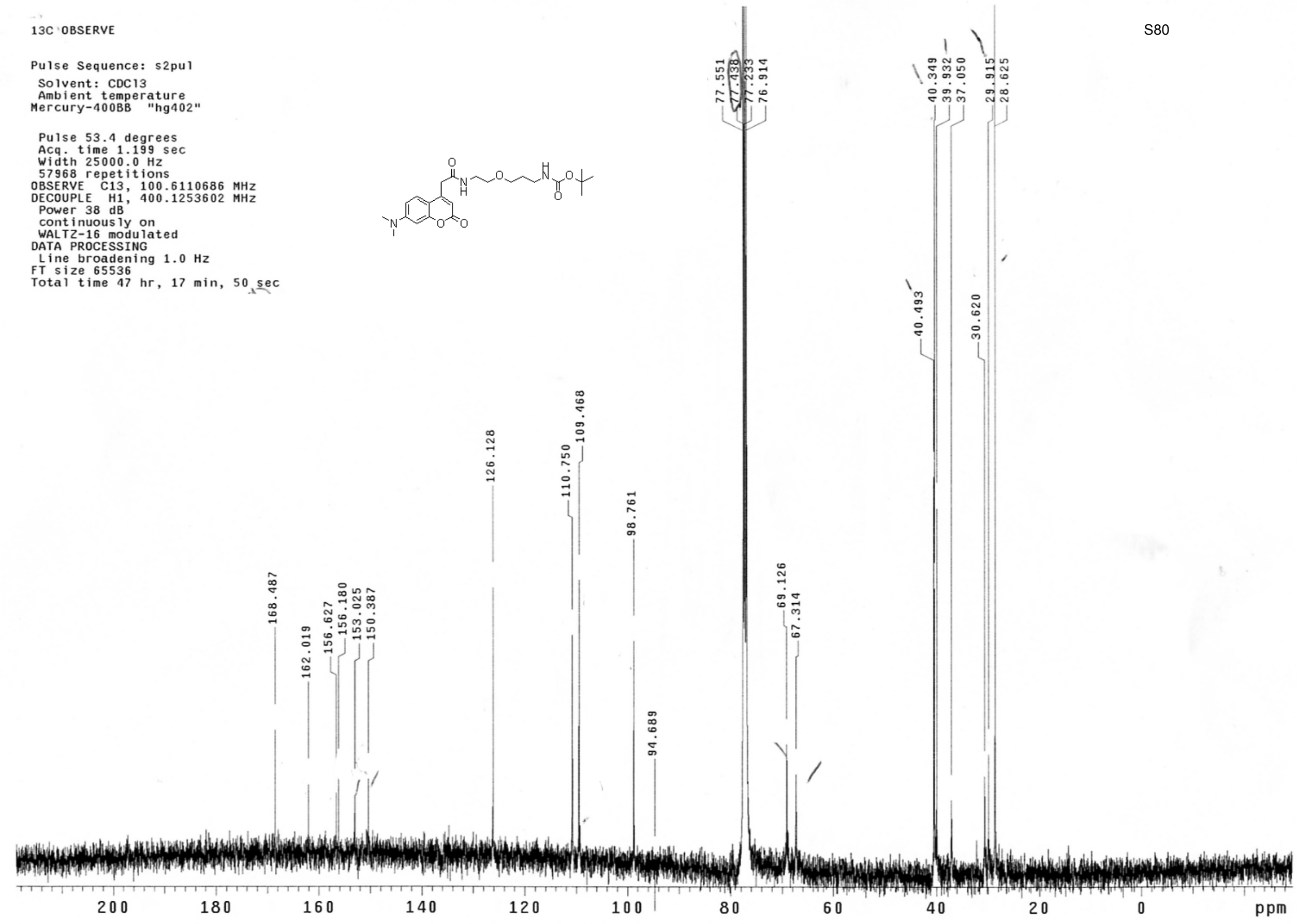
boc deprotection $(2,45) 3.6 .06$

Pulse Sequence: s2pulo

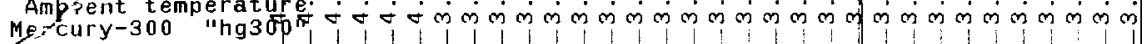

Relax. delay 1,000 sec

Pulse 45.3 degrees

Acq. time 1.995

120 repetitions

DATA PROCESSING

FT size 32768

Total time 0 min, $0 \mathrm{sec}$

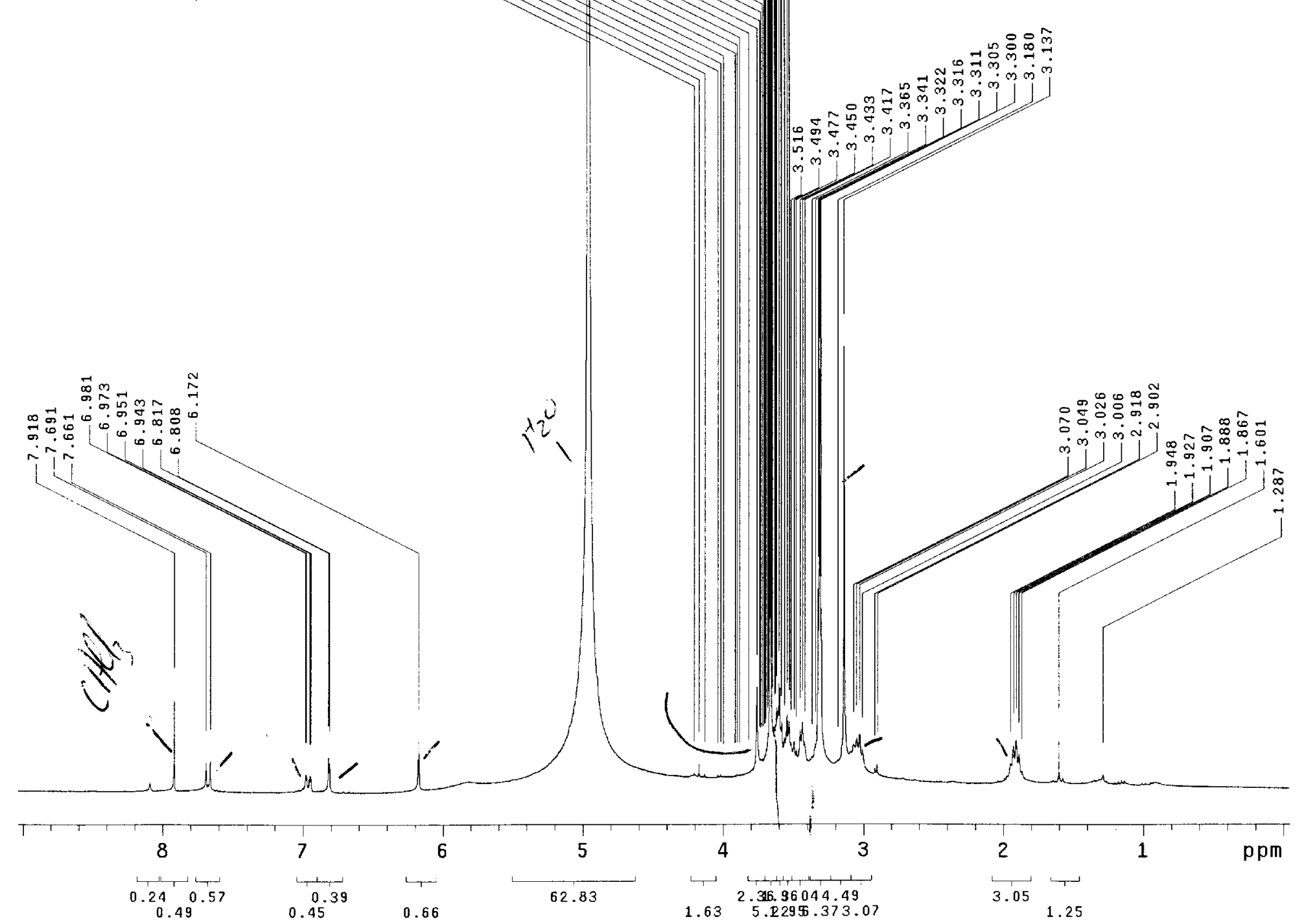


finalproduct $B(2,56) 3.13 .06$

Pulse Sequence: s2pur

Solvent: $\operatorname{CDC} 13$

Ambient temperature
Mercury-400B "hg402"

Relax. delay $1.000 \mathrm{sec}$

Pulse 45.3 degrees

Acq time 1.993 sec

136 repetitions

OBSERVE H1, 400.1233350 MHz

DATA PROCESSING

FT size 32768
Total time $53 \mathrm{~min}, 49 \mathrm{sec}$
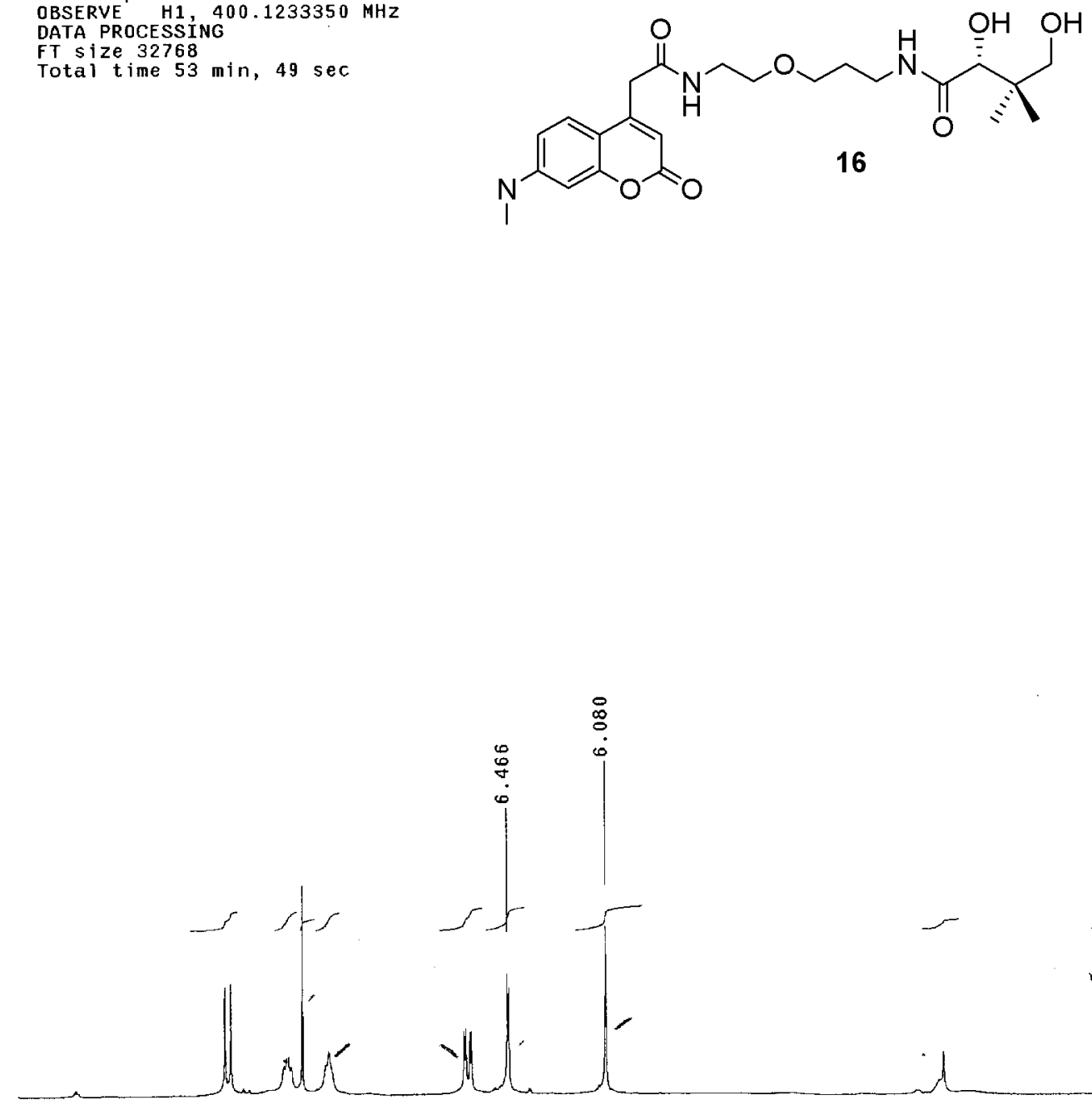

8
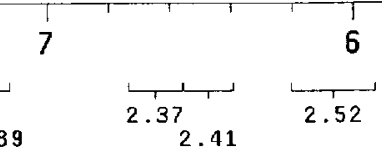

5
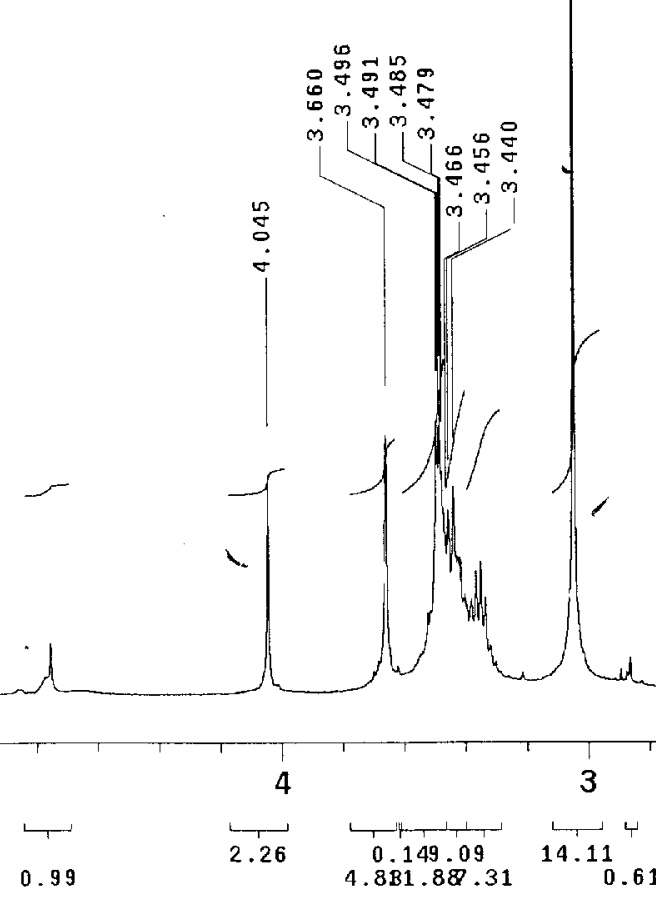
Pulse Sequence: s2pur

Solvent: $\operatorname{Coc} 13$

Ambient temperature
Mercury-400B "ing402"

Pulse 53.4 degrees
Acq. time $1.199 \mathrm{sec}$

A 176 repetitions

OBSERVE C13, 100.6110686 MHZ

DECOUPLE H1, 400.1253602 MHZ

Power $38 \mathrm{~dB}$

cont huously on

WATA PROCESSING

Line broadening $1.0 \mathrm{~Hz}$

FT size 65536

Total time $4 \mathrm{hr}, 4 \mathrm{~min}, 24 \mathrm{sec}$

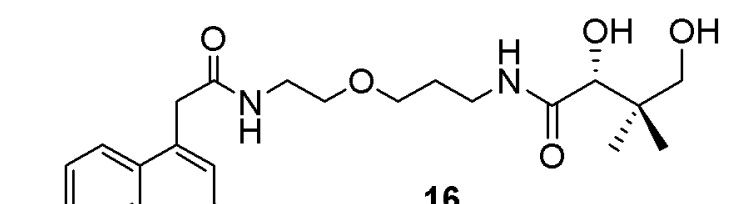

16
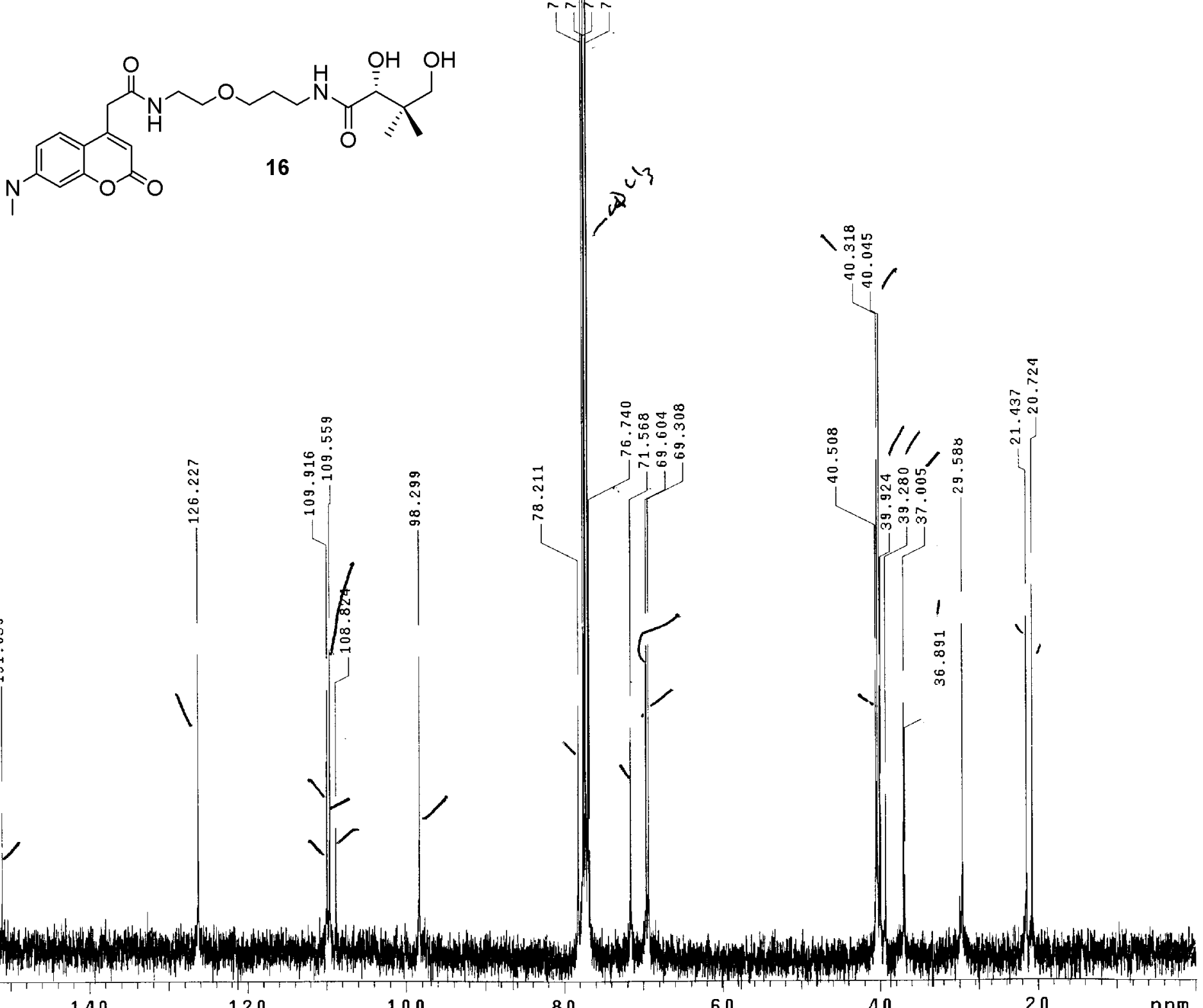

180

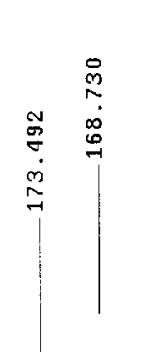

זे.

舀弯

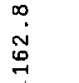

$1 ; \sqrt{2}$

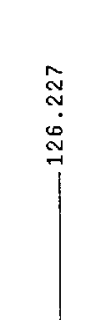

Hand
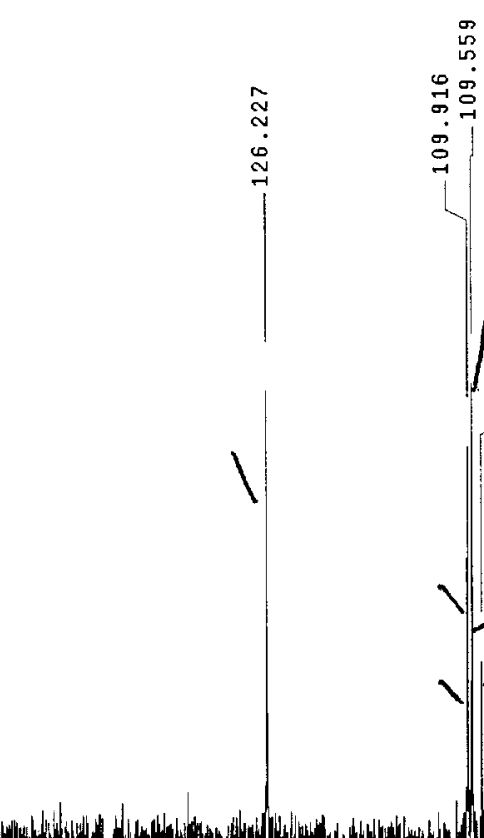

r.
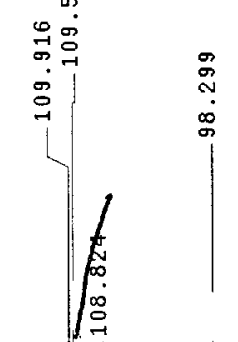

$\stackrel{\mathscr{g}}{\stackrel{9}{0}}$

$\infty$

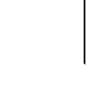

160

140

120

100

80

40

20

ppm 


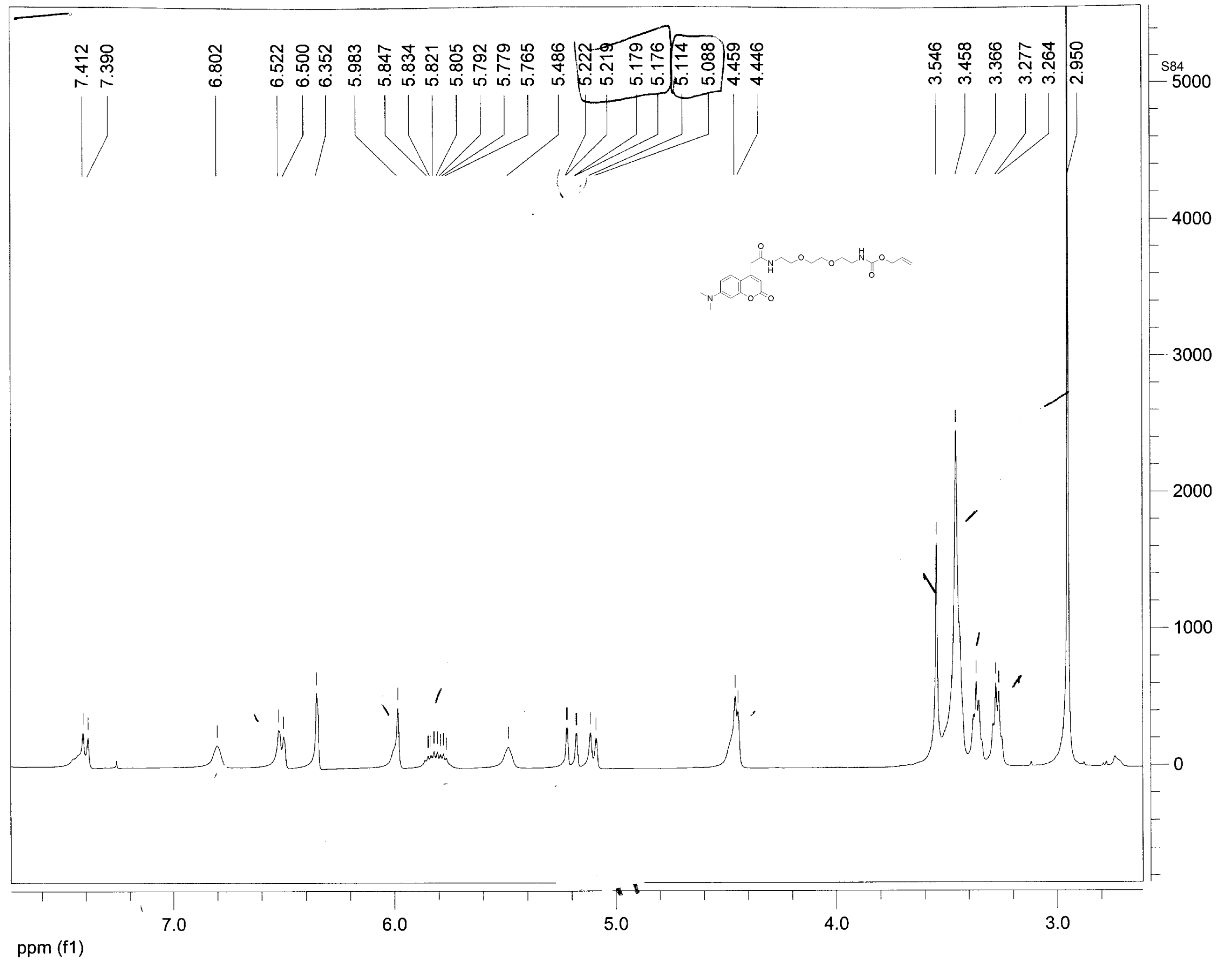




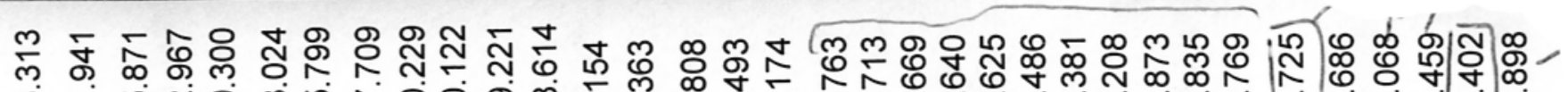

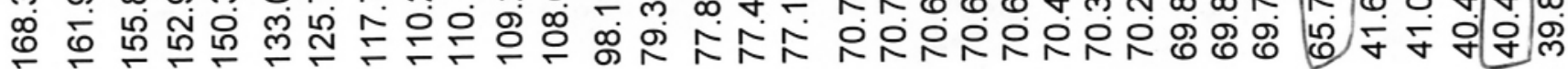

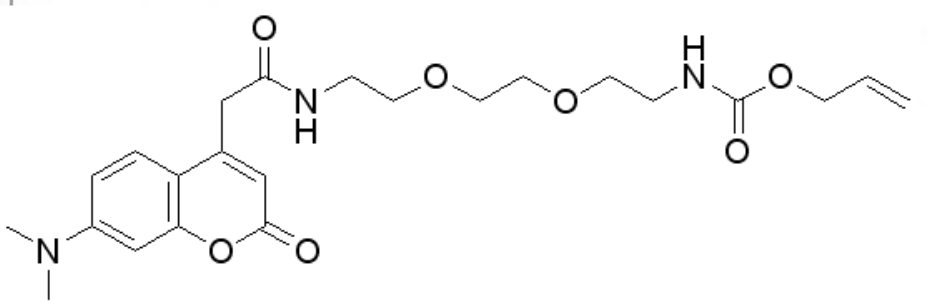


Pulse Sequence: s2pur

Solvent: $\operatorname{CoCl} 3$

Temp. $25.0 \mathrm{C}$ Ć $298.1 \mathrm{~K}$
UNITY-500 "un500"

Pulse 32.5 degrees

Acq. time 1.892 sec

72 repetitions

OBSERVE H1, $500.3087950 \mathrm{MHZ}$

DAA bressini $0.3 \mathrm{~Hz}$

Total time $31 \mathrm{~min}, 42 \mathrm{sec}$
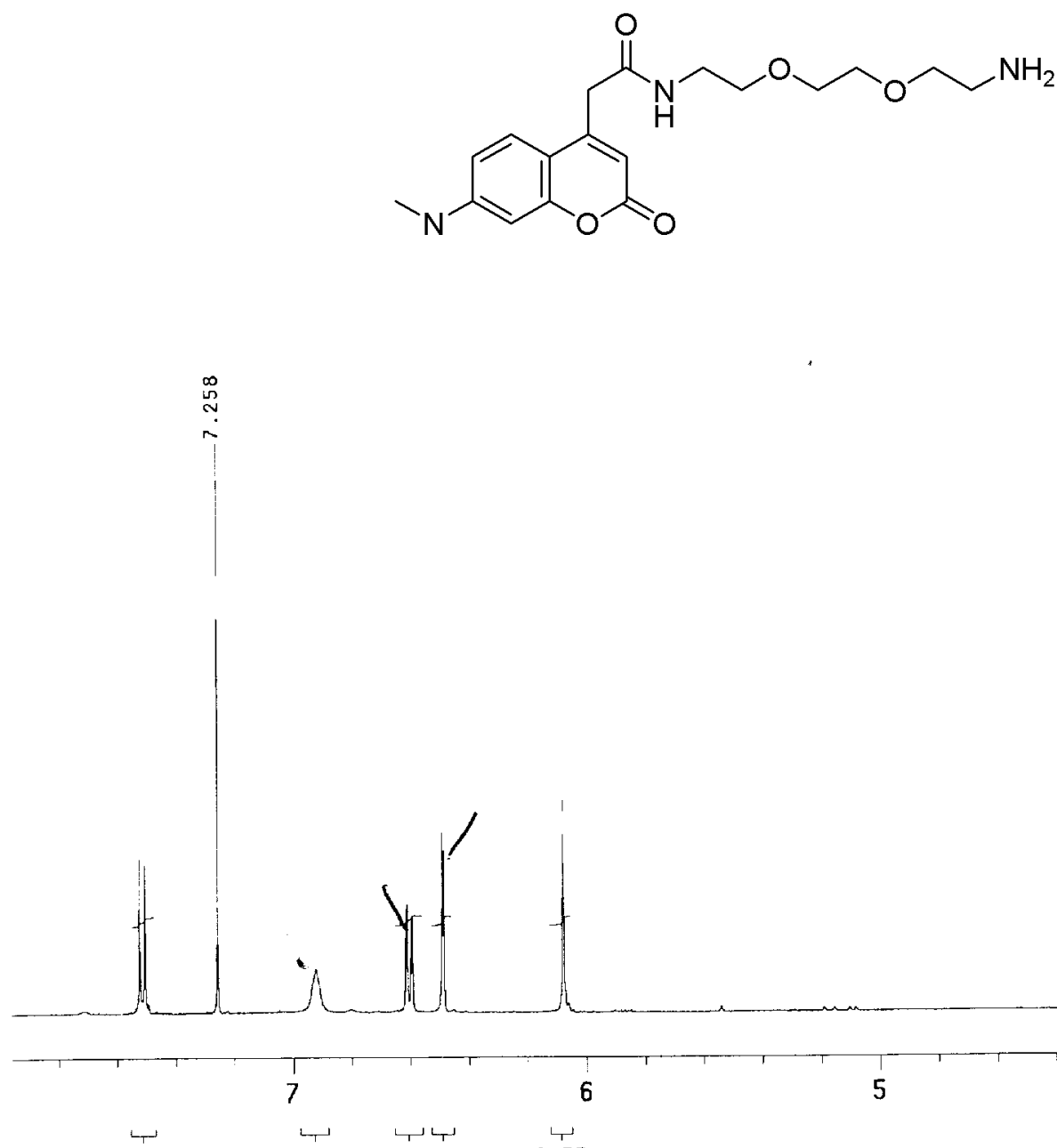

6 


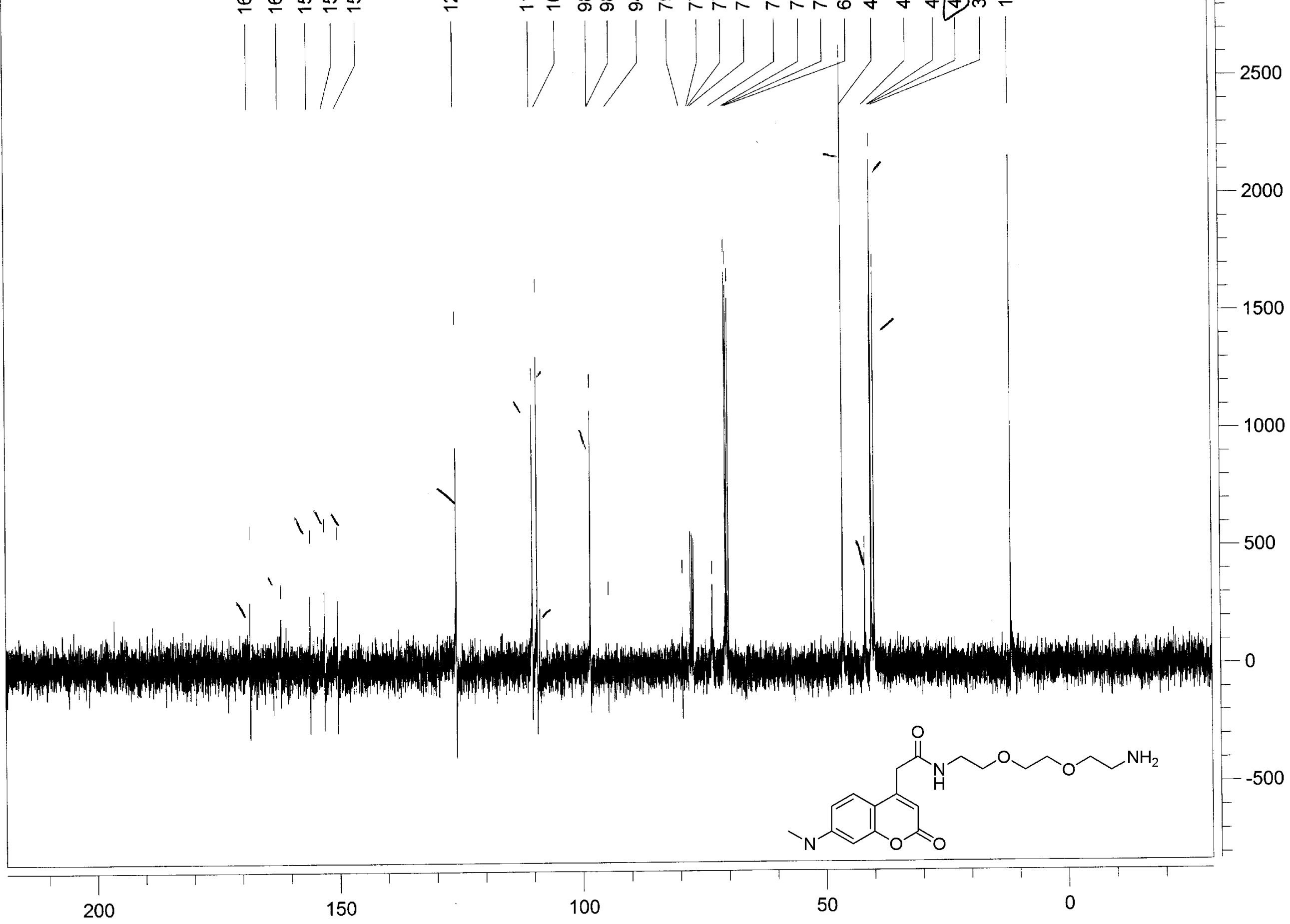




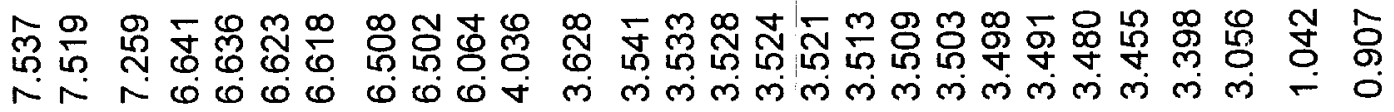

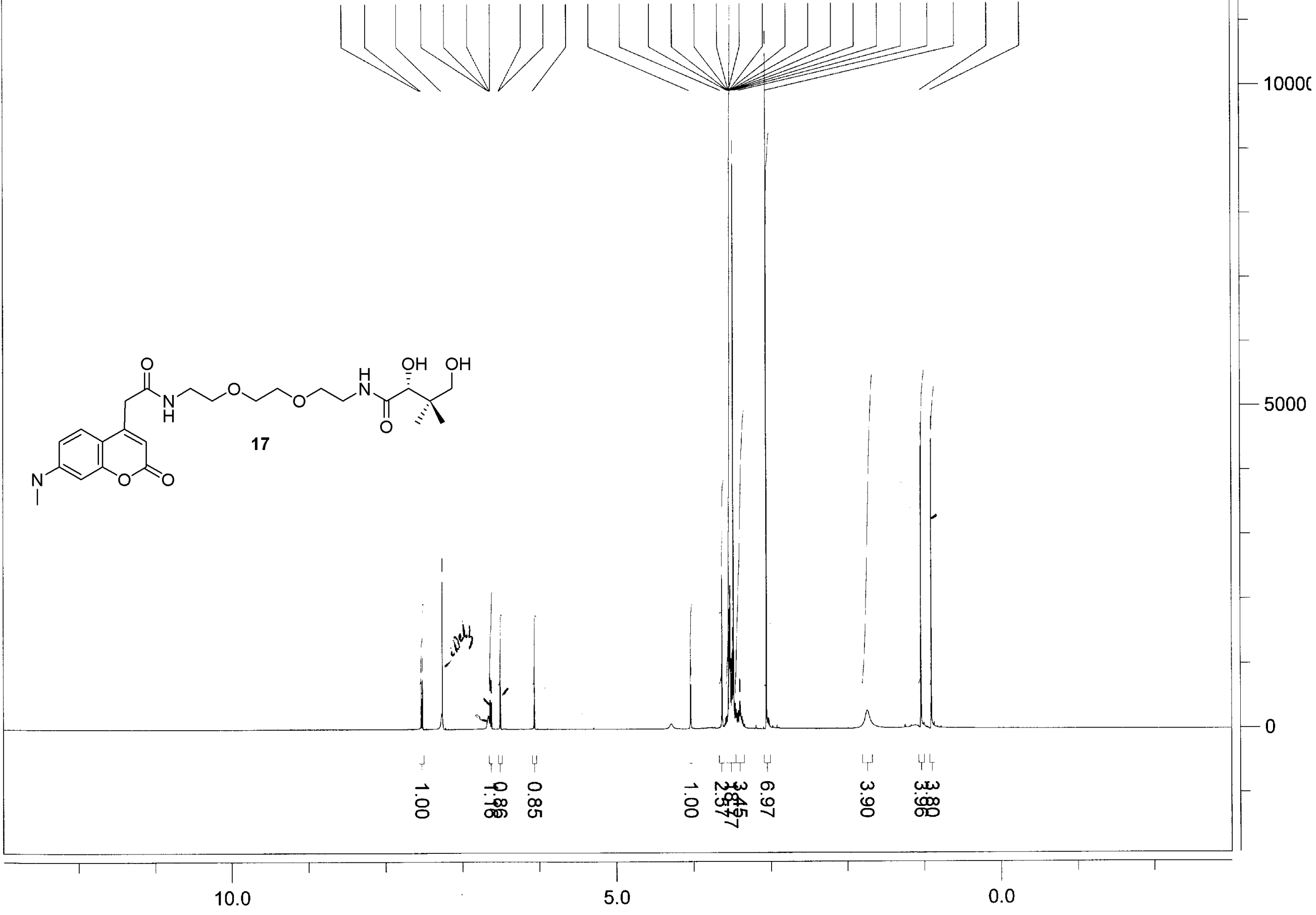


Pulse Sequence: s2pul

Temp. $25.0 \mathrm{C} / 298.1 \mathrm{~K}$
UNITY-500 "un500"

Pulse 51.5 degrees

Acq time $1.892 \mathrm{sec}$

64 repetitions
OBSERVE H1, $500.3087950 \mathrm{MHz}$

Line broadening $0.3 \mathrm{~Hz}$

FT size 32768 min, 42 sec
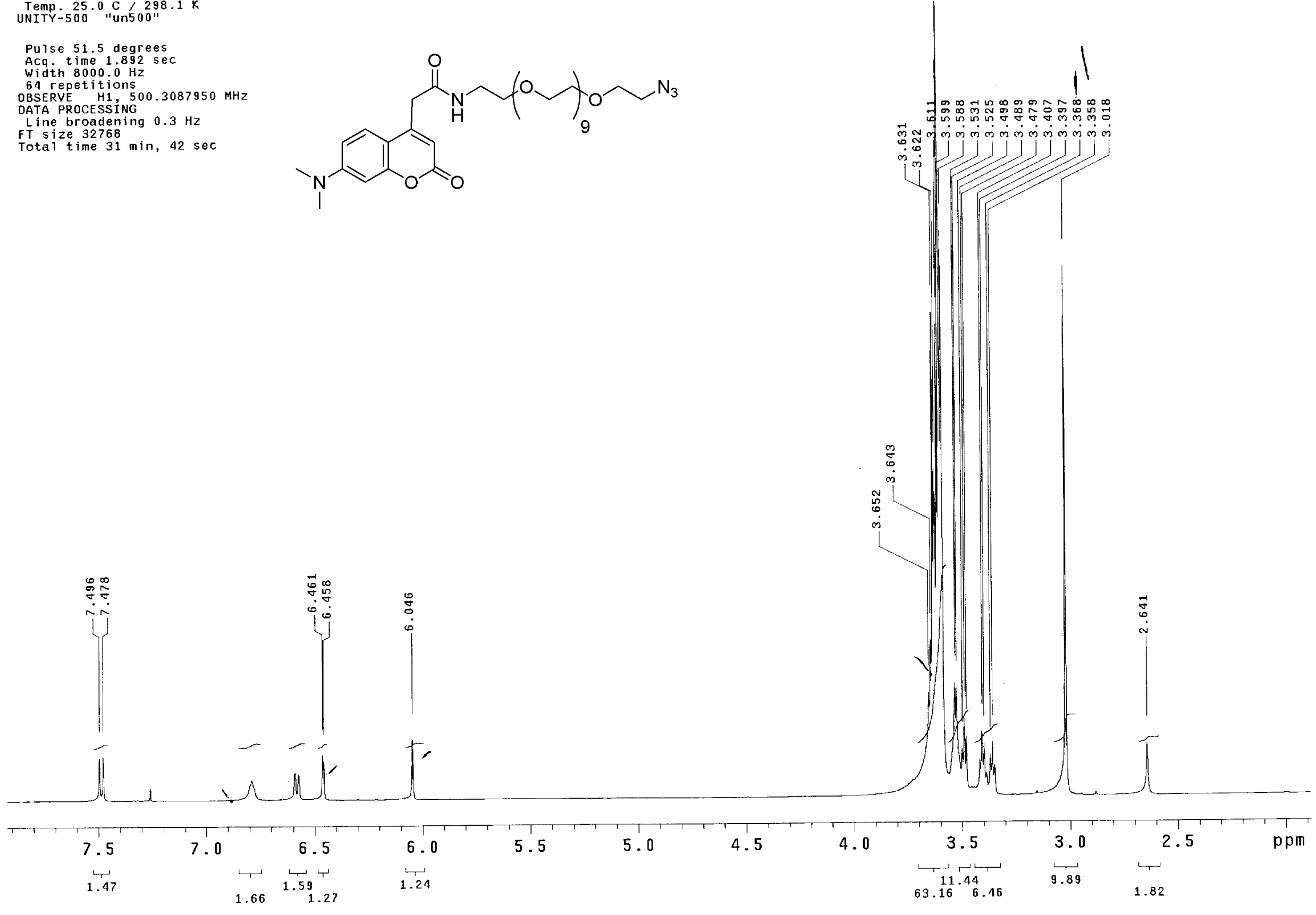
Solvent: $\operatorname{CDC} 13$

Ambient temperature
Mercury-300 "hg300"

Pulse 60.5 degrees

Acq. time 1.815 sec

344 repetitions

OBSERVE C13 $75.4540290 \mathrm{MHZ}$

DECOUPLE H1, 300.0770584 MHZ

cont inuous

WAL TZ-16 modulated

DATA PROCESSING

Line broadening $1.0 \mathrm{~Hz}$

Total time $0 \mathrm{~min}, 0 \mathrm{sec}$
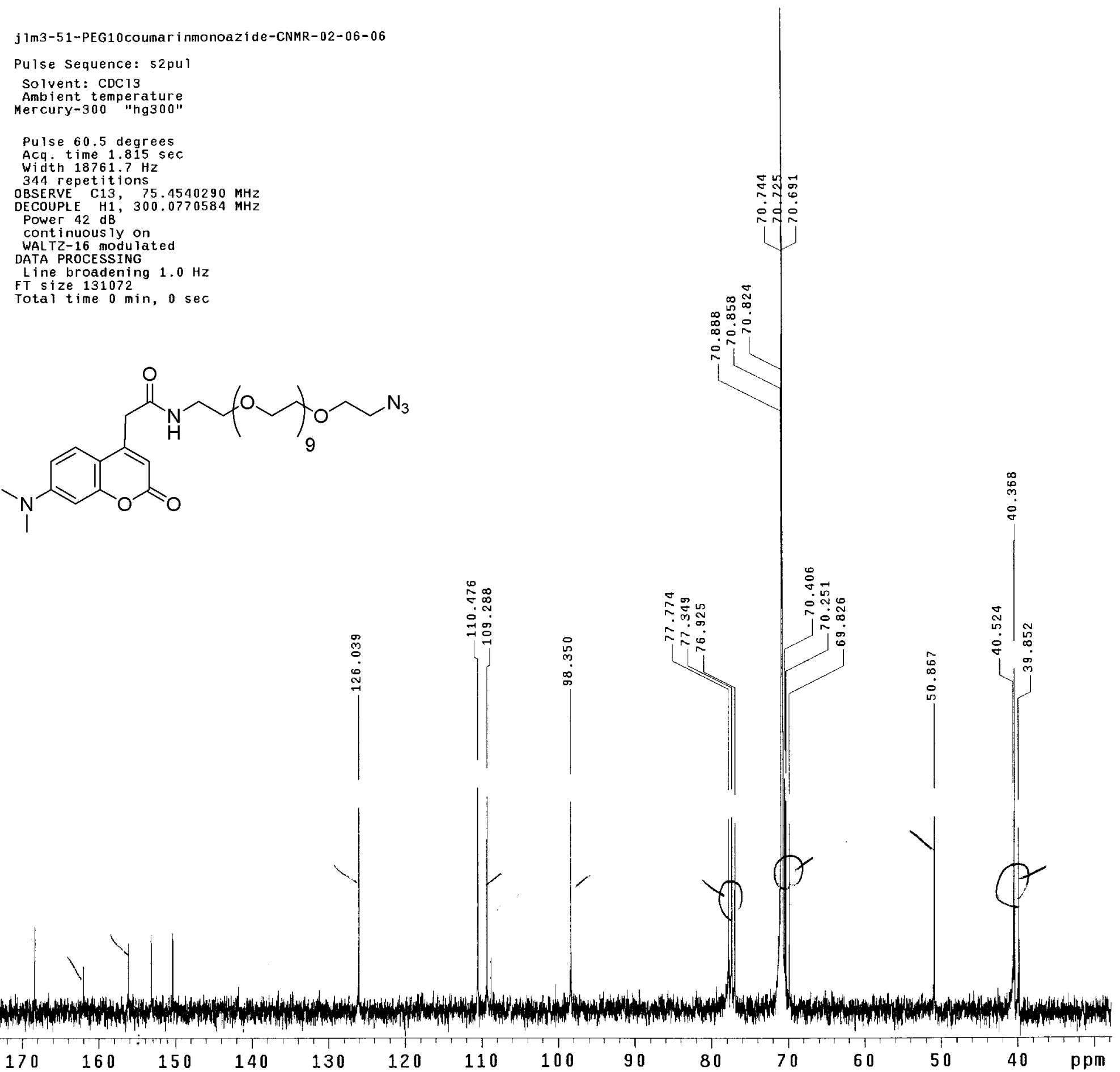
Pulse Sequence: s2pul

Solvent: $\operatorname{coc} 13$

Ambient temperature

mercury-300 "hg300"

Relax. delay $1.000 \mathrm{sec}$

Pulse 45.3 degrees

Acq time 1.995 sec

Width $4506.5 \mathrm{~Hz}$

40 repetitions

OBSERVE H1, 300.0755102 $\mathrm{MHZ}$

DATA PROCESSING

FT size 32768

Total time $0 \mathrm{~min}, 0$ sec

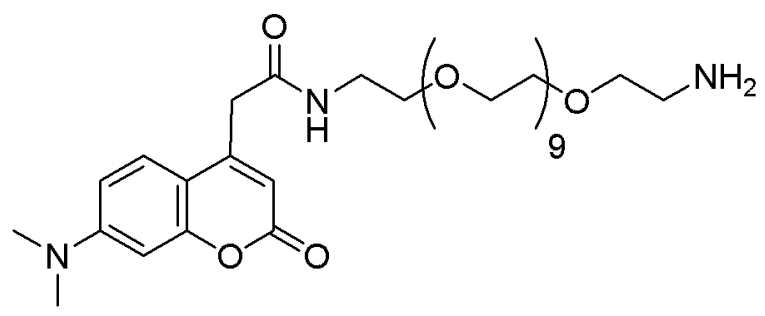


j 1m3-54-PEG1 0 coumarin-staud ingertoam ine-1yoph i lateCNMR-02-07-06

Pulse Sequence: s2pul

Solvent: $\operatorname{cDc} 13$

Ambient temperature

"hg300"

Pulse 60.5 degrees

Acq. time $1.815 \mathrm{sec}$

Width $18761.7 \mathrm{~Hz}$

176 repetitions

OBSERVE C13, 75.4540290 MHZ

DECOUPLE H1; $300.0770584 \mathrm{MHZ}$

Power $42 \mathrm{~dB}$

cont inuous ly on

WALTZ-16 modulated

DATA PROCESSING

Line broadening $1.0 \mathrm{~Hz}$

FT size 131072
Total time $39 \mathrm{~min}, 38 \mathrm{sec}$<smiles>CC(C)OCC(C)(C)OCCNC(=O)Cc1cc(=O)oc2cc(N(C)C)ccc12</smiles>

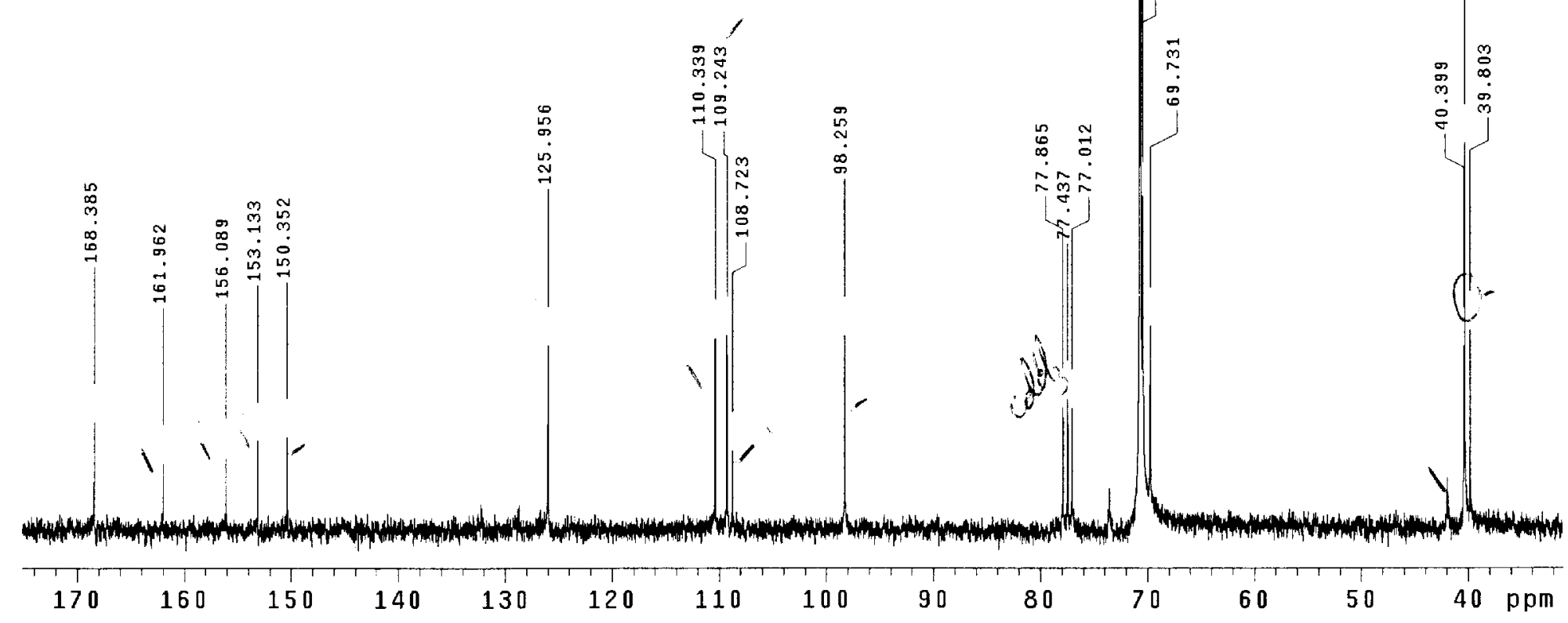




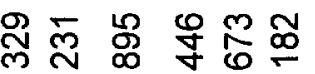

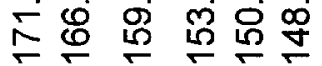

$\stackrel{\infty}{\circ}$

m

స్ల

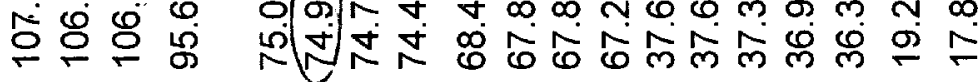
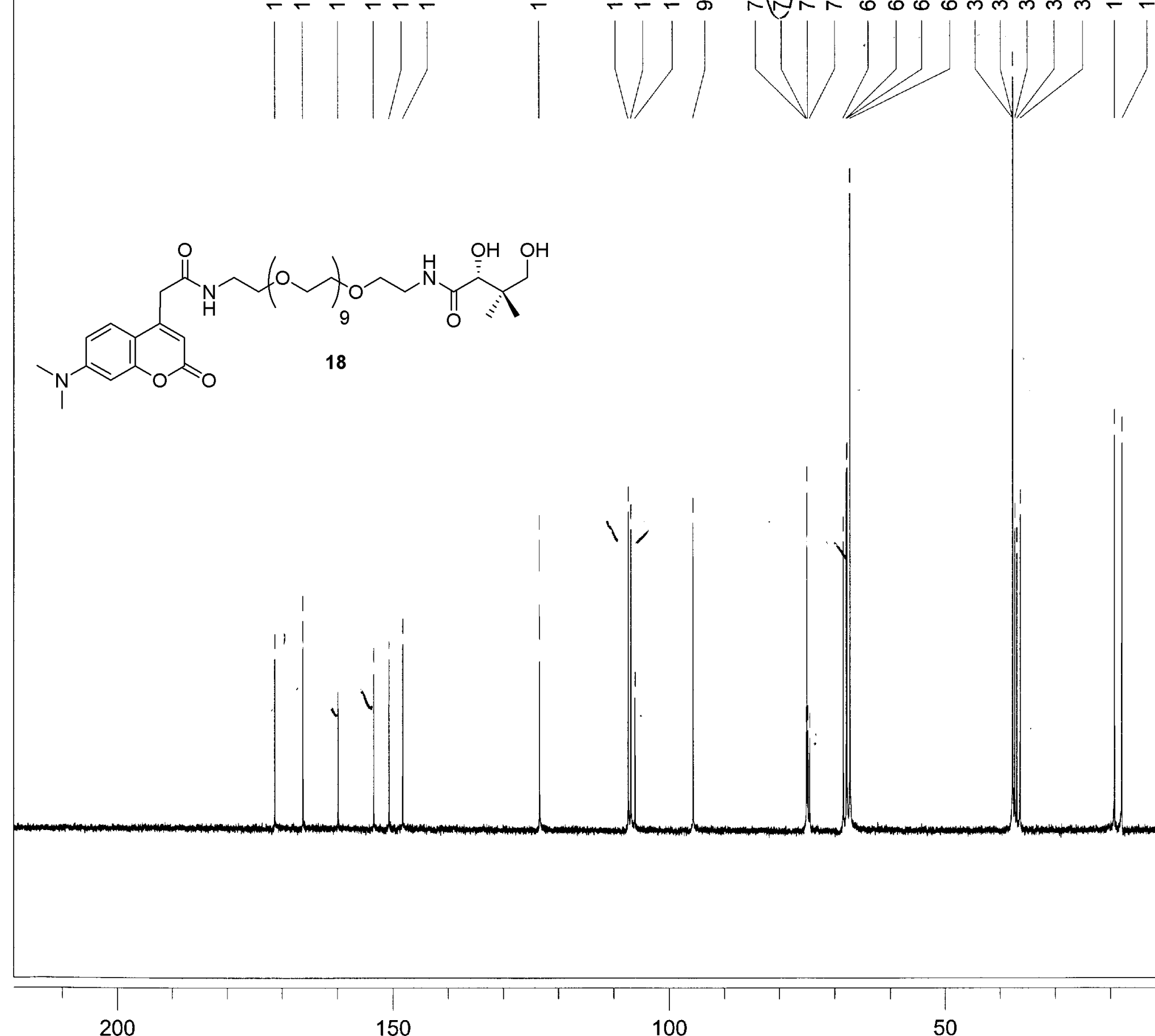

ppm (f1)

150 
nitrile reduction $(2,38) \_2.11 .06$

Pulse Sequence: s2pul

Solvent: $\operatorname{CDC} 13$

Ambient temperature

Relax. delay $1.000 \mathrm{sec}$

Pulse 45.3 degrees

width $6006.0 \mathrm{~Hz}$

88 repetitions

OBSERVE H1, $400.1233350 \mathrm{MHz}$

DATA PROCESSING

Total time $0 \mathrm{~min}, 0 \mathrm{sec}$
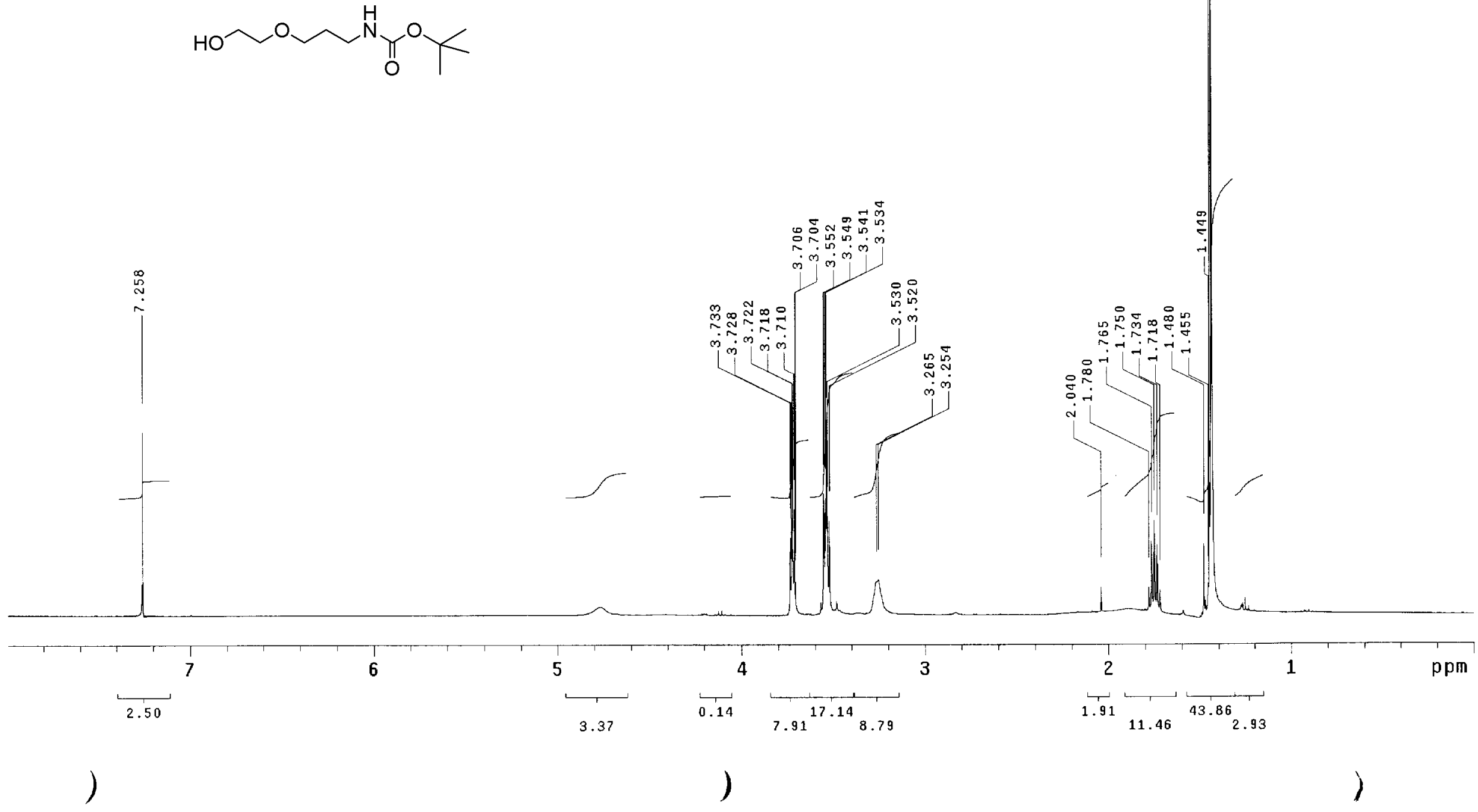
Pulse 60.5 degrees

Acq. time 1.815 sec

Width $18761.7 \mathrm{~Hz}$

1000 repetitions

OBSERVE C13, $75.4540290 \mathrm{MHZ}$

DECOUPLE H1, $300.0770584 \mathrm{MHZ}$

Power 42 dB

cont i nuous ly on

WALTZ-16 modulated

DATA PROCESSING

Line broadening $1.0 \mathrm{~Hz}$

FT size 131072

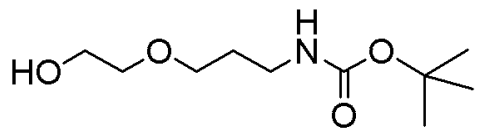

Total time 35 min, 14 sec
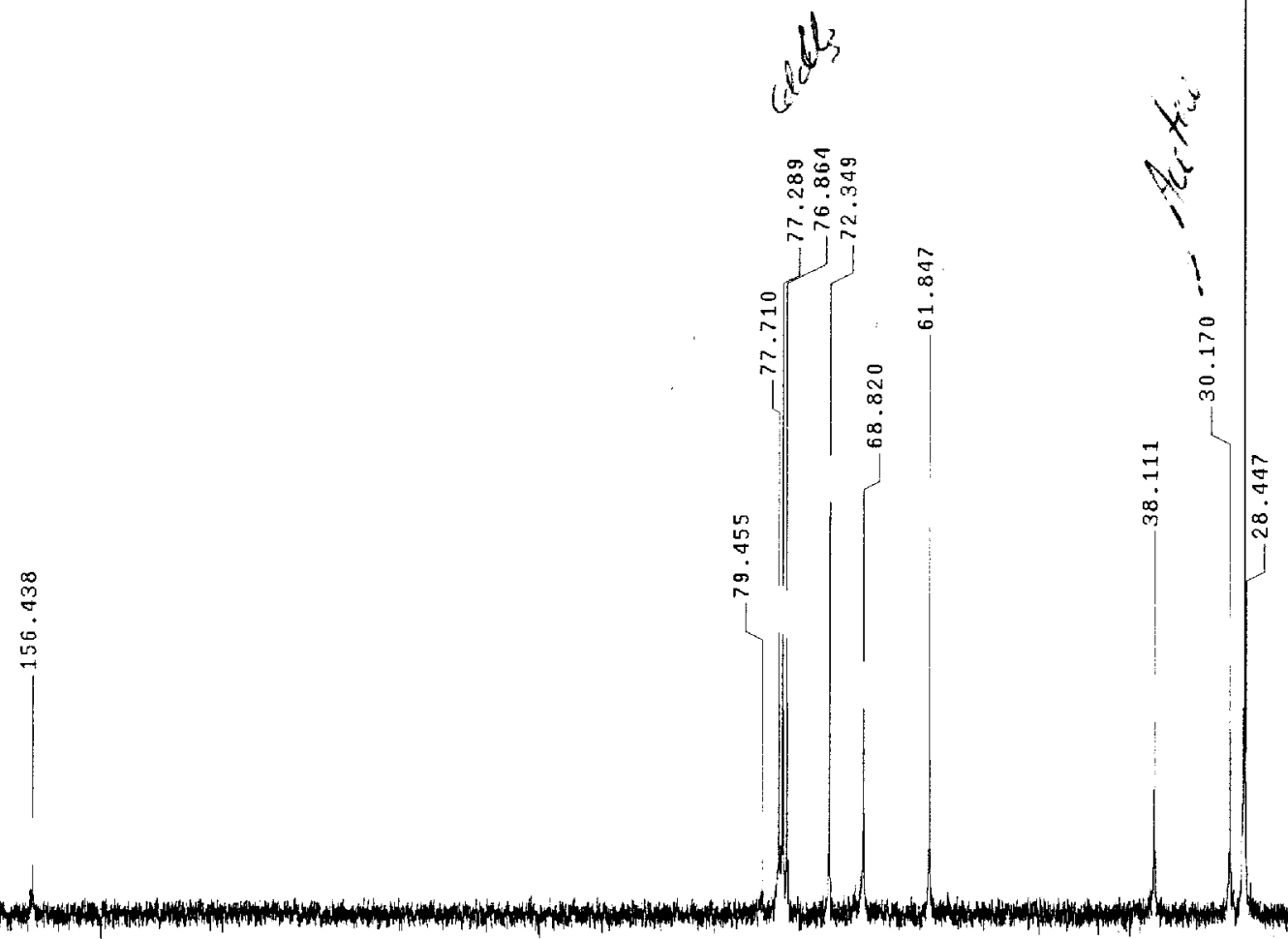

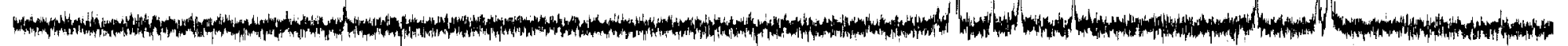


Ambient temperature

Mercury-300 "hg300"

Relax. delay $1.000 \mathrm{sec}$

Pulse 45.3 degrees

Acq. time 1.995 sec

Width $4506.5 \mathrm{~Hz}$

72 repetitions

OBSERVE H1, 300.0766925 $\mathrm{MHz}$

DATA PROCESSING

FT Size 32768

Total time $53 \mathrm{~min}, 11 \mathrm{sec}$
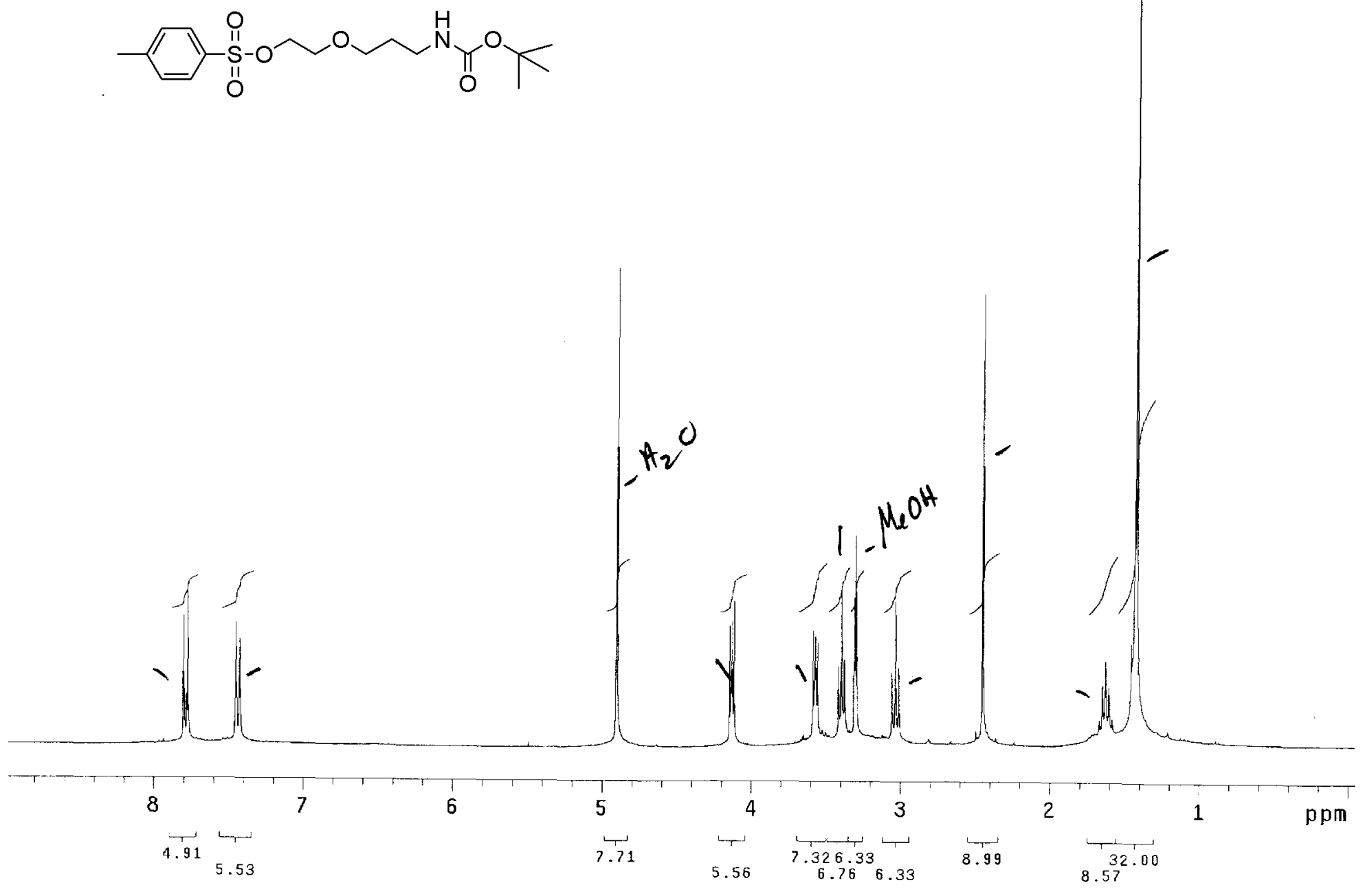
Ambient temperature
Mercury- 300 "hg $300 "$

Pulse 60.5 degrees

Acq. time $1.815 \mathrm{sec}$

$100018761.7 \mathrm{~Hz}$

OBSERVE C13, 75.4540290 MHz

H1, 300.0770584 MHZ

WALTZ-16 modulated

DATA PROCESSING

Line broadening $1.0 \mathrm{~Hz}$

Total time $35 \mathrm{~min}, 14 \mathrm{sec}$

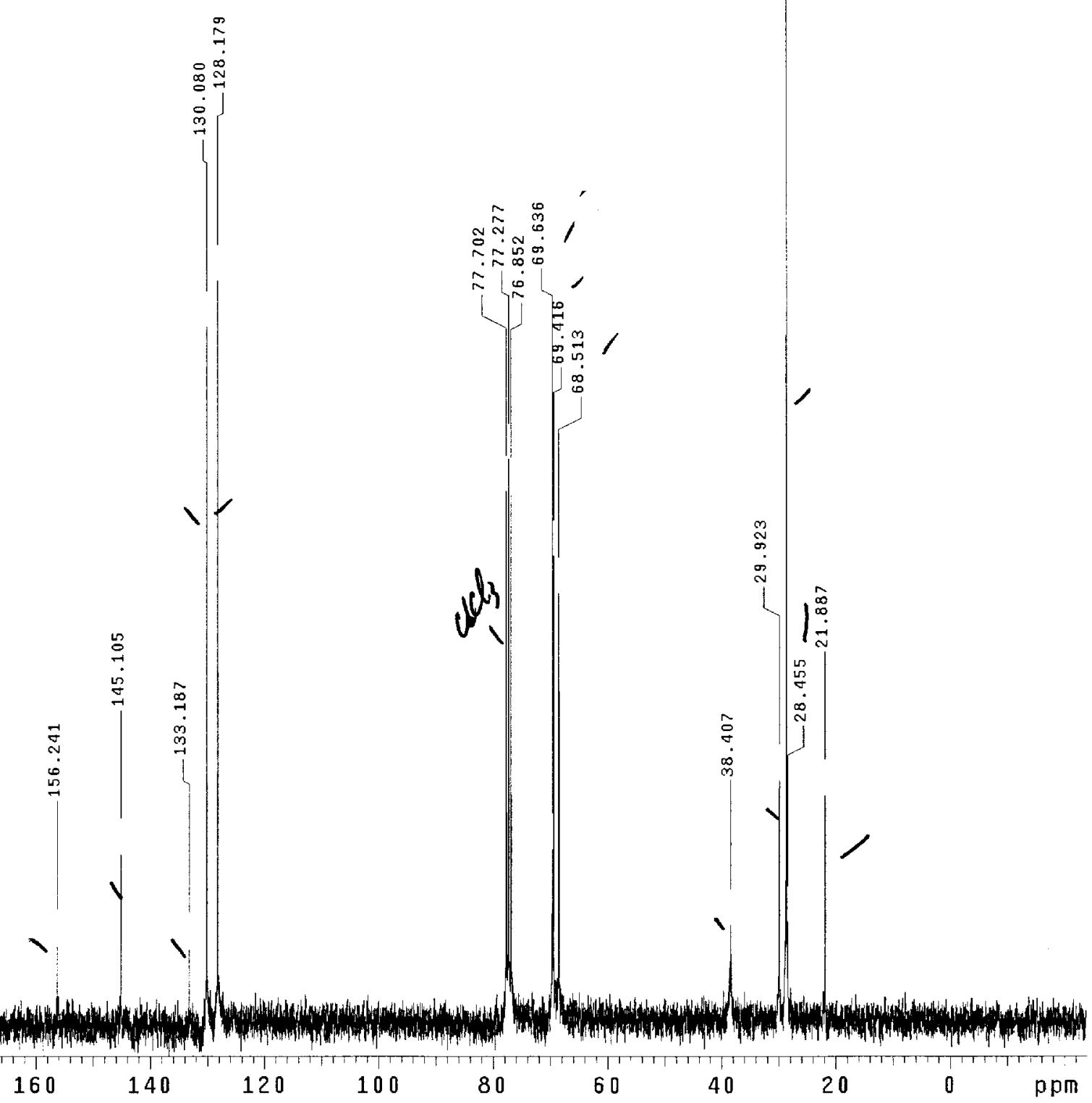


Pulse 51.5 degrees

Acq time 1.892 sec

72 repetitions

OBSERVE H1, $500.3087950 \mathrm{MHZ}$

DATA PROCESSING

Line broadening $0.3 \mathrm{~Hz}$

Total time $31 \mathrm{~min}, 42 \mathrm{sec}$

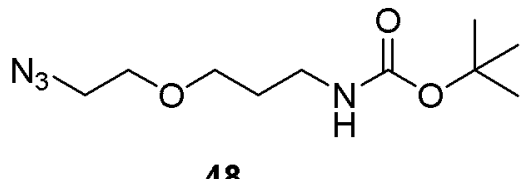

48

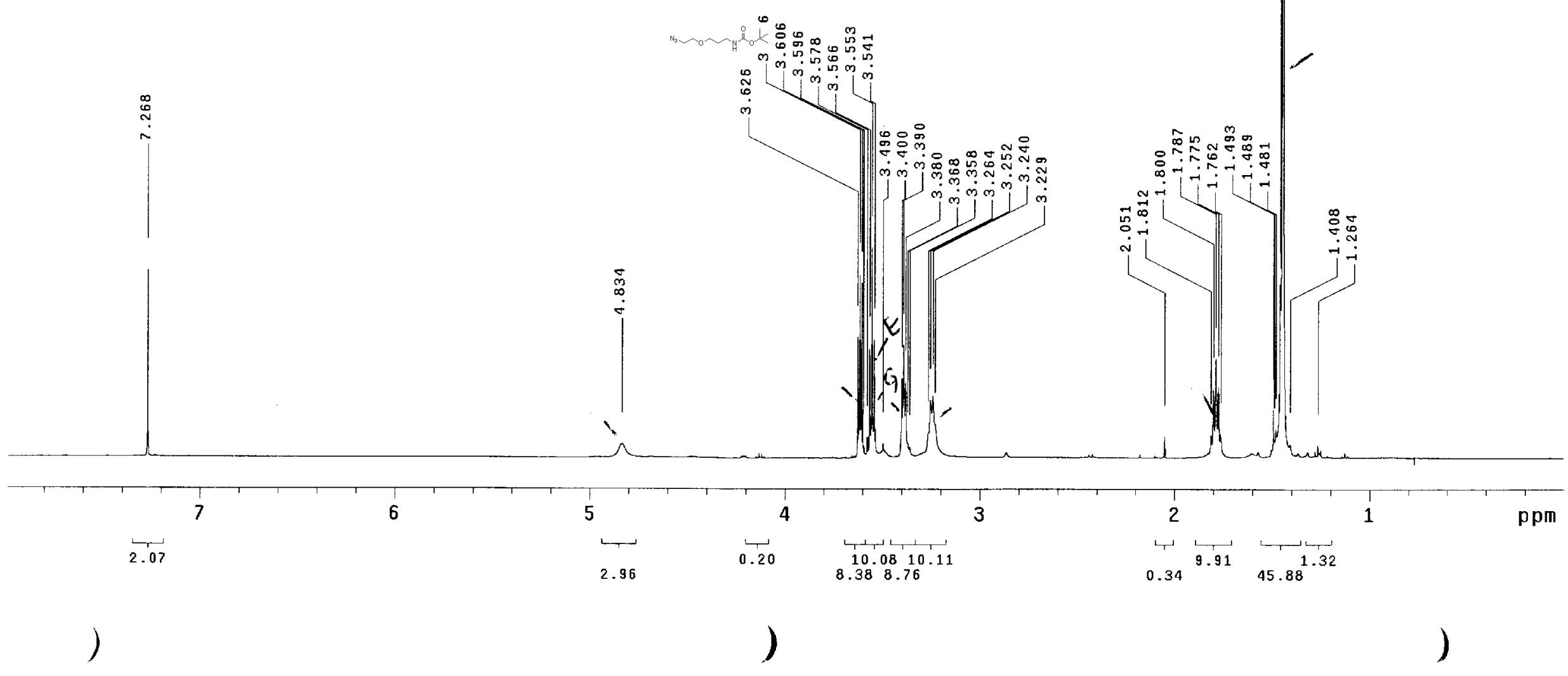


Pulse sequence: s2pul

Solvent: $\operatorname{CDC} 13$

Ambient temperature
Mercury-300 "hg300"

Pulse 60.5 degrees

Acq. time $1.815 \mathrm{sec}$

Width $18761.7 \mathrm{~Hz}$

1328 repetitions
OBSERVE C13, 75.4540290 MHz

DECOUPLE H1, 300.0770584 MHz

Power $42 \mathrm{~dB}$

cont inuous ly on

WAL $2-16$ modulated

DATA PROCESSING

Line broadening $1.0 \mathrm{~Hz}$

FT size 131072

Total time $5 \mathrm{hr}, 52 \mathrm{~min}, 1 \mathrm{sec}$<smiles>CC(C)(C)OC(=O)NCCCOCCN</smiles>

48

$\stackrel{\sim}{\sim}$

i.

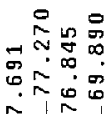

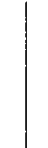

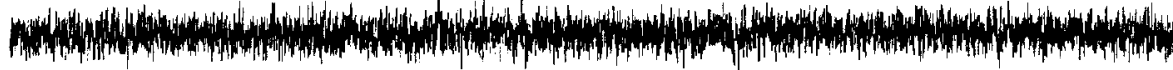
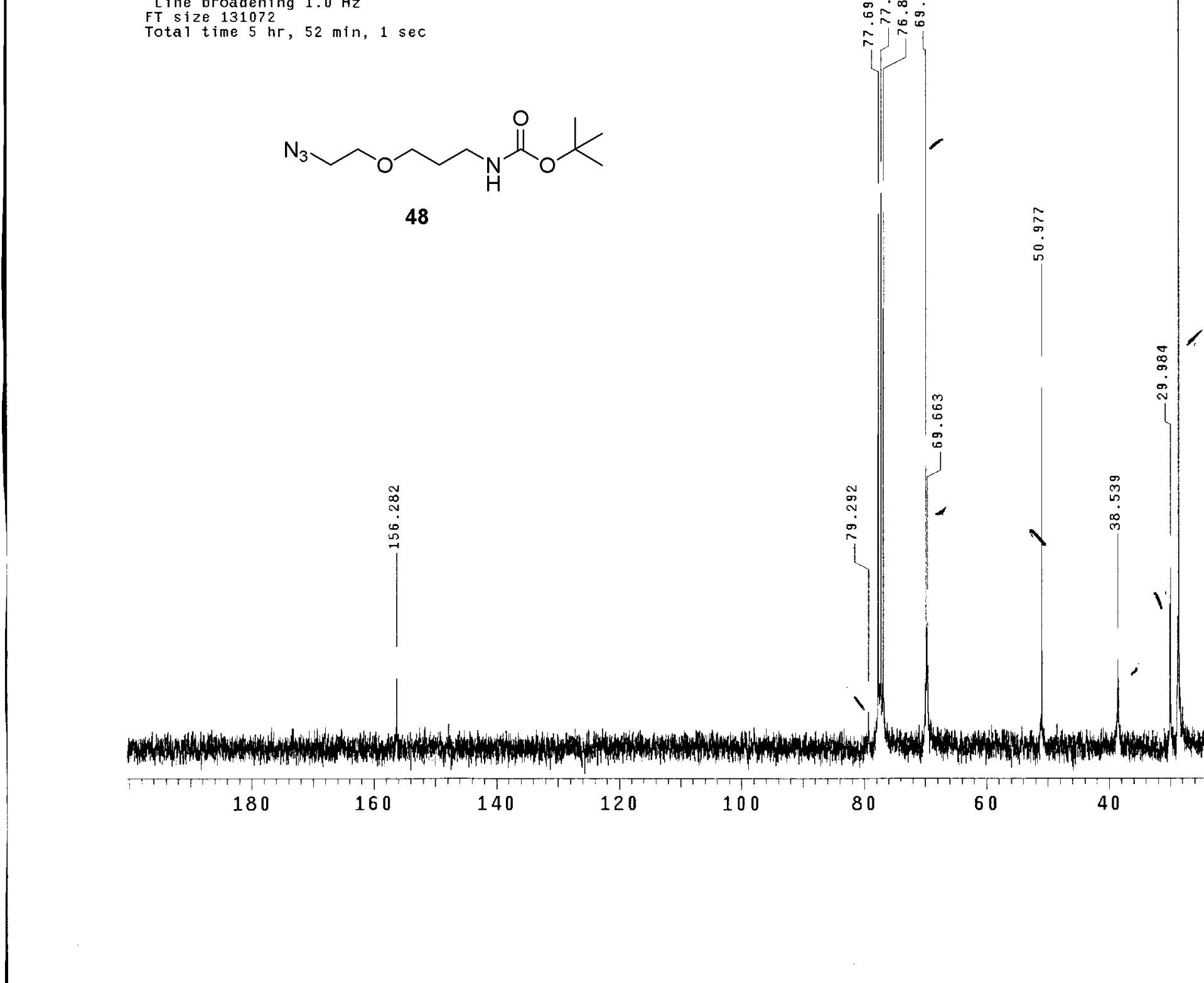

160

140

120

100

80

20

$\mathrm{ppm}$ 